\title{
Preditores ótimos baseados em amostragem com dois estágios de populações finitas
}

\author{
Silvina San Martino \\ Tese apresentada ao \\ Instituto de Matemática e Estatística \\ da Universidade de São Paulo \\ para obtenção do grau de \\ Doutor em Estatística \\ Área de Concentração: Estatística \\ Orientador: Prof. Dr. Julio da Motta Singer
}

Durante a elaboração deste trabalho, a autora recebeu apoio financeiro do $\mathrm{CNPq}$

São Paulo

2005 


\section{Preditores ótimos baseados em amostragem com dois estágios de populações finitas}

Este exemplar corresponde à redação final da tese devidamente corrigida e defendida por Silvina San Martino e aprovada pela comissão julgadora.

São Paulo, dezembro de 2005.

Comissão Julgadora:

- Prof. Dr. Julio da Motta Singer (Orientador) - IME-USP

- Prof. Dr. Heleno Bolfarine - IME-USP

- Profa. Dra. Édina Miazaki - UnB

- Profa. Dra. Denise Britz do Nascimento Silva - IBGE

- Prof. Dr. Fernando Moura - UFRJ 
À minha família, pela compreensão, apoio, paciência e amor. 


\section{Agradecimentos}

Sou muito grata a todos aqueles que compartilharam comigo esta etapa e que, em maior ou menor medida, me acompanharam, apoiaram e permitiram este logro.

Ao meu orientador, o Prof. Julio Singer, de quem aprendi muito e a quem agradeço a disposição e a dedicação para me atender sempre, a confiança e o apoio em todas as etapas da tese e o estímulo para desenvolver novas idéias. E também ao Prof. Ed Stanek pelo seu aporte ao desenvolvimento deste trabalho.

Ao CNPq e a Facultad de Ciencias Agrarias (UNMdP, Argentina) que me auxiliaram financeiramente durante este período.

Aos professores do Dpto. de Estatística do IME-USP, assim como aos funcionários, pessoal da CPG, da biblioteca e da secretaria do MAE, pelo trato sempre agradável e cordial e pela ajuda que me dispensaram sempre.

À minha família que, apesar dos momentos difíceis que passamos, sempre me apoiou e me incentivou neste empreendimento. E à Mariela, a quem conheci no começo desta etapa, mas com quem compartilhamos alegrias e dificuldades que fizeram dela hoje minha "amiga-irmã". Seu carinho e suporte fizeram que este caminho fosse mais simples, agradável e interessante.

Ao todos os meus colegas no IME (muitos na verdade) e ao grupo argentino, pela sua generosidade, cordialidade e afeição que fizeram mais gratos os momentos tanto de estudo quanto de diversão. A Gustavo e Carolina que me ajudaram muito no início e, em especial, a Felipe, Jorge, Susana e Florencia, que me brindaram seu afeto sincero e com os quais, ainda que de diferente forma, tivemos muitas e boas conversas de amigos e foram de grande apoio nesta etapa.

Aos meus colegas na Facultad de Ciencias Agrarias, em especial Gloria, Petra e Gaby que me brindaram sua desinteressada colaboração. E aos amigos que deixei na Argentina, os quais, ainda na distância, me acompanharam afetuosamente. 


\section{Resumo}

Neste trabalho propomos preditores empíricos do valor latente de grupos, sob o modelo de permutação aleatória, recentemente proposto por Stanek e Singer (2004, JASA, 99: 1119-1130), e o modelo de Scott e Smith (1969, JASA, 64: 830-840), baseados em amostragem com dois estágios de populações finitas. Comparamos o desempenho dos preditores ou preditores empíricos, tanto de variáveis contínuas quanto discretas com ou sem erro na resposta, obtidos sob estes modelos para populações finitas com aqueles obtidos sob modelos para populações infinitas (linear misto ou linear misto generalizado) por meio de um estudo de simulação. Quando as componentes de variância são conhecidas, este estudo mostra que o preditor obtido sob o modelo de permutação aleatória geralmente apresenta erro quadrático médio mínimo, tanto para variáveis contínuas quanto discretas e variâncias dentro dos grupos iguais ou diferentes. No caso dos preditores empíricos, aquele obtido sob o modelo de permutação aleatória resulta o mais estável, sendo umas vezes aquele que apresenta o menor erro quadrático médio outras vezes equivalente com ele. Aliás, seu erro quadrático médio nunca supera em mais de um $50 \%$ àquele do melhor preditor, a diferênça de seus competidores. Também obtemos estimadores da resposta média ou total e preditores ótimos do valor latente de grupos sob o modelo de permutação aleatória em situações em que há covariáveis presentes, quando a amostragem é feita com dois estágios em uma população finita agrupada. 


\section{Abstract}

We propose empirical predictors of cluster latent values based on the random permutation model, recently proposed by Stanek and Singer (2004, JASA, 99: 11191130), and the Scott and Smith model (1969, JASA, 64: 830-840) for two-stage random sampling from finite populations. We develop a simulation study to compare the performance of predictors and empirical predictors either for continuous or discrete responses with or without error, obtained under these models for finite populations with those for infinite populations (linear and generalized linear mixed models). When variance components are known, the random permutation model predictor generally shows minimum mean square error, for continuous or discrete responses and equal or different within cluster variances. The random permutation model based empirical predictor is more stable than its competitors, being the best predictor (i.e. with minimum mean square error) or equivalent to it. Moreover, its mean square error never exceeds in more than $50 \%$ that of the best predictor, differently from its competitors. We also derive optimal estimators of population parameters (such as the total or mean response) and predictors of cluster latent values from a random permutation model with auxiliary information, under twostage random sampling from a clustered finite population. 


\section{Sumário}

1 Introdução 1

1.1 Motivação . . . . . . . . . . . . . . . . . . . . . 1

1.2 Proposta de pesquisa . . . . . . . . . . . . . . . . 4

2 Preditores ótimos para variáveis contínuas $\quad 6$

2.1 Introdução . . . . . . . . . . . . . . . . . . . . 6

2.2 Definições básicas . . . . . . . . . . . . . . . . . . . . 8

2.3 Modelos para populações finitas . . . . . . . . . . . . . . 8

2.3.1 O modelo de permutação aleatória $(M P A) \ldots \ldots . \ldots 8$

2.3.2 O modelo de Scott e Smith $(M S S) \ldots \ldots \ldots$

$2.4 \mathrm{O}$ modelo linear misto $(M L M)$ para populações infinitas . . . . . . 12

2.5 Preditores de características populacionais . . . . . . . . . . . 13

2.5.1 Definição dos preditores . . . . . . . . . . . . . 13

2.5 .2 Comparação dos preditores . . . . . . . . . . . . . . . 14

2.6 Preditores empíricos . . . . . . . . . . . . . . . . . . 17

2.7 Estudo por simulação . . . . . . . . . . . . . . . . . . . . . . . . . . 19

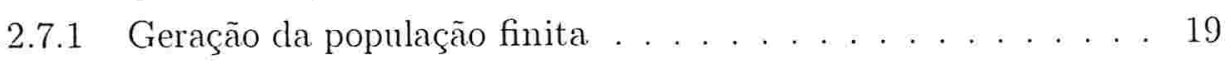

2.7 .2 Amostragem em dois estágios . . . . . . . . . . . . . . 20

2.7 .3 Preditores . . . . . . . . . . . . . . . . . 22

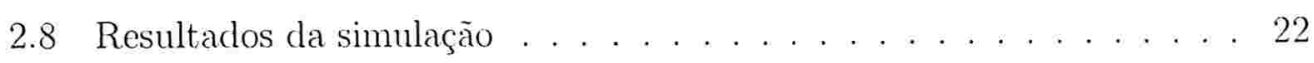

2.8.1 Variâncias intra-grupos iguais . . . . . . . . . . 23

2.8.2 Variâncias intra-grupo diferentes . . . . . . . . . . . . 29

2.9 Discussão e conclusões . . . . . . . . . . . . . . . . . . . 33 
3 Preditores ótimos para variáveis discretas 35

3.1 Introdução . . . . . . . . . . . . . . . . . 35

3.2 O modelo linear misto generalizado $(M L M G)$ para populações infinitas 36

3.3 Preditores . . . . . . . . . . . . . . . . . . . . 37

3.4 Estudo por simulação . . . . . . . . . . . . . . . . . . . . 38

3.4 .1 Geração da população finita . . . . . . . . . . . . . . . 38

3.4 .2 Amostragem em dois estágios . . . . . . . . . . . . . 39

3.4 .3 Preditores . . . . . . . . . . . . . . . . . . 40

3.5 Resultados . . . . . . . . . . . . . . . . . . . . 40

3.5.1 Componentes de variância conhecidas . . . . . . . . . . . . 41

3.5.2 Componentes de variância desconhecidas . . . . . . . . . . . 42

3.6 Discussão e conclusões . . . . . . . . . . . . . . . . . . . . 45

4 Estimadores e preditores ótimos em presença de covariáveis $\quad 47$

4.1 Introdução . . . . . . . . . . . . . . . . . . . . . . . 47

4.2 Amostragem em dois estágios . . . . . . . . . . . . . . 48

4.2 .1 Definições básicas . . . . . . . . . . . . . . . . . . . . . 48

4.2.2 Modelo não-estocástico para uma variável resposta e $p$ covariáveis . . . . . . . . . . . . . . . . . 5 51

4.2.3 Modelo estocástico para uma variável resposta e $p$ covariáveis 51

4.2 .4 Estimação . . . . . . . . . . . . . . . . . . . . 53

4.2.5 Incorporação da informação adicional das covariáveis . . . . 55

4.2.6 Especificação de elementos amostrados e não amostrados . . . 55

4.2.7 Estimação do total populacional usando informação das covariáveis . . . . . . . . . . . . . . . . . 57

4.2.8 Predição dos valores latentes dos grupos amostrados usando informação das covariáveis . . . . . . . . . . . . . . . 60

4.2 .9 Estimadores e preditores empíricos . . . . . . . . . . 63

4.3 Amostragem estratificada . . . . . . . . . . . . . . . 65

4.3 .1 Definiçōes básicas . . . . . . . . . . . . . . . . 65

4.3.2 Modelo não-estocástico para uma variável resposta e $p$ covariáveis . . . . . . . . . . . . . . . . 66

4.3.3 Modelo estocástico para uma variável resposta e $p$ covariáveis 67 
4.3.4 Incorporação da informação adicional das covariáveis . . . . 68

4.3.5 Especificação de elementos amostrados e não amostrados . . . 69

4.3.6 Estimação da resposta total populacional e em cada estrato usando informação das covariáveis . . . . . . . . . . . 70

5 Conclusões $\quad 74$

5.1 Considerações finais . . . . . . . . . . . . . . . . . . . . . . . . 74

5.2 Futuras pesquisas . . . . . . . . . . . . . . . 76

A Preditores e preditores empíricos - Resultados adicionais $\quad 77$

A.1 Comparação das constantes de encolhimento . . . . . . . . . . . 77

A.2 Obtenção de preditores empíricos . . . . . . . . . . . . 78

A.2.1 Modelo de Permutação Aleatória . . . . . . . . . . . . . 79

A.2.2 Modelos de Scott e Smith e Linear Misto . . . . . . . . . . 80

B Resultados da simulação $\quad 85$

B.1 Tabelas adicionais . . . . . . . . . . . . . . . . . . 85

B.2 Figuras adicionais . . . . . . . . . . . . . . . . . 91

C Detalhes sobre a obtenção de estimadores e preditores ótimos em presença de covariáveis 95

C.1 Amostragem em dois estágios . . . . . . . . . . . . . . . 95

C.1.1 Momentos de $\boldsymbol{Y}$ e $\boldsymbol{X}^{(k)} \ldots \ldots \ldots \ldots \ldots 9 . \ldots . \ldots 9$

C.1.2 Momentos de $Z$. . . . . . . . . . . . . . . . 100

C.1.3 Momentos induzidos para a parte amostrada e não amostrada 100

C.1.4 Predição dos valores latentes dos grupos amostrados usando informação das covariáveis . . . . . . . . . . . . . . . 106

C.2 Amostragem estratificada . . . . . . . . . . . . . . . . . 109

C.2.1 Momentos de $Z_{s}$ e $Z$. . . . . . . . . . . . . 109

C.2.2 Especificação de elementos amostrados e não amostrados . . . 110

C.2.3 Estimação da resposta total em cada estrato usando informação das covariáveis . . . . . . . . . . . . . . 112

Referências Bibliográficas $\quad 119$ 


\section{Lista de Tabelas}

2.1 Características das populações simuladas e dos planos amostrais . . . 21

2.2 Preditores com desempenho semelhante ao melhor preditor $(A R P<$ $5 \%$ ), para as populações com variâncias intra-grupos iguais . . . . . 25

2.3 Estatísticas descritivas do acréscimo relativo percentual $(A R P)$ no EQME dos preditores empíricos com relação ao EQMS dos preditores obtidos sob cada modelo, para as populações com variâncias intragrupos iguais . . . . . . . . . . . . . . 2

2.4 Porcentagem de casos em que os dois melhores preditores empíricos apresentam EQME mínimo, para as populações com variâncias intragrupos iguais . . . . . . . . . . . . . . . . . . 28

2.5 Preditores empíricos com desempenho semelhante ao melhor preditor empírico $(A R P<15 \%)$, para as populações com variâncias intragrupos iguais . . . . . . . . . . . . . . . . 28

2.6 Preditores com desempenho semelhante ao melhor preditor $(A R P<$ $5 \%)$, para as populações com variâncias intra-grupos diferentes . . . . 30

2.7 Estatísticas descritivas do acréscimo relativo percentual $(A R P)$ no EQME dos preditores empíricos com relação ao EQMS dos preditores obtidos sob cada modelo, para as populações com variàncias intragrupos diferentes . . . . . . . . . . . . . . . . . 31

2.8 Porcentagem de casos em que os dois melhores preditores empíricos apresentam EQME mínimo, para as populações com variâncias intragrupos diferentes 
3.1 Porcentagem de ocorrências de EQME mínimo para o preditor empírico com melhor desempenho (populações com variâncias intra-grupos di-

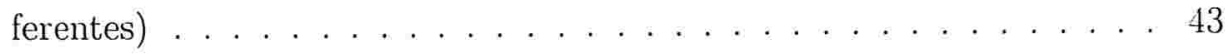

3.2 Porcentagem de ocorrências de EQME mínimo para os dois preditores empíricos com melhor desempenho (populações com variâncias intragrupos iguais) . . . . . . . . . . . . . . 4

B.1 Máximo acréscimo relativo percentual $(A R P)$ no EQMS para a $M G$ e os preditores obtidos sob o $M L M$, o $M S S$ e o $M P A$ com relação ao EQMS do melhor preditor, para as populações com variâncias intragrupos iguais . . . . . . . . . . . . 8

B.2 Bom desempenho de cada preditor, para as populações com variâncias intra-grupos iguais . . . . . . . . . . . . . 86

B.3 Desempenho ruim de cada preditor, para as populações com variâncias intra-grupos iguais . . . . . . . . . . . . . . 87

B.4 Máximo acréscimo relativo percentual $(A R P)$ no EQMS para a $M G$ e os preditores obtidos sob o $M L M$, o $M S S$ e o $M P A$ com relação ao EQMS do melhor preditor, para as populações com variâncias intragrupos diferentes . . . . . . . . . . . . . . . . . 88

B.5 Bom desempenho de cada preditor, para as populações com variâncias intra-grupos diferentes . . . . . . . . . . . . . . . . . . 89

B.6 Desempenho ruim de cada preditor, para as populações com variâncias intra-grupos diferentes . . . . . . . . . . . . . . . . 90 


\section{Lista de Figuras}

2.1 Comportamento das constantes de encolhimento . . . . . . . . 16

B.1 Acréscimo relativo percentual no EQMS da $M G$ com relação ao EQMS do melhor preditor, para as populações com $N=10, M=20 \mathrm{e}$ variâncias intra-grupos iguais e conhecidas . . . . . . . . . . 91

B.2 Acréscimo relativo percentual no EQMS do preditor obtido sob o $M L M$ com relação ao EQMS do melhor preditor, para as populações com $N=10, M=20$ e variâncias intra-grupos iguais e conhecidas . . 92

B.3 Acréscimo relativo percentual no EQMS do preditor obtido sob o MSS com relação ao EQMS do melhor preditor, para as populações com $N=10, M=20$ e variâncias intra-grupos iguais e conhecidas. . . . . 93

B.4 Acréscimo relativo percentual no EQME dos preditores empíricos com relação ao EQMS dos preditores obtidos sob o $M L M$, o $M S S$ ou o MPA, para as populações com variàncias intra-grupos iguais . . . . 94 


\section{Capítulo 1}

\section{Introdução}

\subsection{Motivação}

Populações finitas agrupadas são comumente encontradas na prática. Filhos em famílias, turmas em escolas, ambulatórios em hospitais, etc. são alguns exemplos típicos. Quando um dos objetivos é predizer os valores latentes associados aos grupos selecionados, i.e., as respostas médias esperadas para as unidades que constituem cada grupo, a inferência geralmente é baseada em uma amostragem aleatória com dois estágios sem reposição. Dependo do estudo, a variável resposta de interesse pode ser tanto contínua quanto discreta. Como exemplo podemos citar o trabalho do Sistema de Avaliação de Rendimento Escolar do Estado de São Paulo (SARESP) em que o interesse é avaliar a habilidade em Português (considerada como variável contínua e estimada por meio da Teoria de Resposta ao Item) dos alunos de certa série. Nesse caso, a população objetivo é finita e agrupada, sendo que as escolas correspondem aos grupos e os alunos, aos indivíduos. Um dos objetivos é predizer a habilidade esperada da escola. Nestes estudos, além da variável resposta, geralmente são consideradas covariáveis tanto para os grupos quanto para os indivíduos e a idéia é melhorar as prediçōes com base nessa informação adicional. Um outro exemplo corresponde a pesquisas feitas através de questionários anônimos para determinar o uso de drogas por estudantes de uma certa universidade. Aqui as faculdades podem corresponder aos grupos e os alunos aos indivíduos, mas a variável resposta estudada é discreta. por exemplo, uma contagem (número de vezes por semana que se usam 
drogas) ou uma variável binária (usa ou não usa drogas).

O problema de predizer os valores latentes dos grupos é importante tanto nestes quanto em muitos outros problemas práticos. Quando se assume que a variável em estudo é normal, os preditores tipicamente usados são os baseados na teoria dos modelos lineares mistos (ver Goldberger (1962), Robinson (1991), McCulloch e Searle (2001), entre outros). Nestes modelos assume-se, além da normalidade da variável resposta, que os dados foram escolhidos de uma população infinita (ou finita mas muito grande). Num enfoque mais geral, a variável resposta pode pertencer à família exponencial de distribuições, que engloba variáveis contínuas e discretas. Estes modelos são os denominados modelos lineares mistos generalizados (ver McCulloch e Searle (2001), Fahrmeir e Tutz (1994), por exemplo). Embora mais abrangente com respeito à distribuição da variável resposta, esse modelo assume que a população de interesse é infinita. Além disso, as inferências são geralmente baseadas em resultados assintóticos, o que requer um tamanho amostral grande para sua aplicação.

Nem os modelos lineares mistos nem os modelos lineares mistos generalizados levam em consideração o fato de que a amostra é colhida numa população finita. Dois enfoques que consideram esse aspecto da população são:

1. O enfoque freqüentista ou baseado no planejamento (Cassel et al. (1977), Cochran (1977), Särndal et al. (1992)) em que as constantes (parâmetros) associadas aos indivíduos da população finita são consideradas não estocásticas e a inferência se baseia exclusivamente no processo de amostragem.

2. O enfoque preditivo ou baseado em modelos, em que se assume que a população objetivo é uma realização de um conjunto de variáveis aleatórias denominada superpopulação (Bolfarine e Zacks (1992), Valliant et al. (2000)), não leva em conta o planejamento amostral e sob o qual a inferência é fundamentada em técnicas de predição.

Sob este segundo enfoque, o modelo desenvolvido por Scott e Smith (1969) e estendido por Bolfarine e Zacks (1992) para incluir erros na resposta, permite predizer os valores latentes associados aos grupos quando a amostragem é feita $\mathrm{cm}$ dois estágios a partir de uma população finita. Este enfoque tem sido pouco usado devido. cm parte, à estrutura artificial da superpopulação, à sua sensibilidade à 
incorreta especificação do modelo e à carência de software que o implemente computacionalmente.

Além disso, vários esforços têm sido feitos para vincular os dois enfoques e achar estimadores com boas propriedades e interpretáveis sob ambos (Brewer et al. (1988), Brewer (1999), Little (2004)). Recentemente, Stanek, Singer e Lencina (2004) propuseram um método baseado no planejamento (para amostragem aleatória simples), que usa as técnicas preditivas sob um modelo de permutação aleatória. O método foi estendido por Stanek e Singer (2004) para predizer os valores latentes associados aos grupos, sob esquemas de amostragem em dois estágios balanceada que também permite a incorporação de erro na resposta.

Duas vantagens deste modelo, além de considerar a estrutura finita da população, são sua natureza intuitiva e sua simplicidade, sendo o único que faz uma ligação direta entre os parâmetros da população finita e um conjunto de variáveis aleatórias induzidas pelo esquema amostral. Como o modelo probabilístico é baseado no planejamento, não necessita suposições distribucionais além da existência dos momentos de primeira e segunda ordem. Portanto, pode ser usado em uma. ampla gama de situações práticas, tanto para variáveis contínuas quanto discretas.

Em geral, os preditores dos valores latentes dos grupos sob os diferentes modelos são obtidos da soma ponderada da resposta das unidades observadas e de preditores da resposta das não observadas, mas diferem com respeito aos pesos ou fatores de encolhimento (shrinkage constants). Estes fatores dependem das componentes de variância populacionais, das características da população finita (como o tamanho do grupo) e do esquema de amostragem. Na prática, as variâncias raramente são conhecidas e precisam ser estimadas, obtendo-se assim preditores empíricos. Em determinadas situações, os preditores (ou os preditores empíricos) obtidos sob os diferentes modelos podem diferir bastante e é essencial contar com alguma ferramenta que permita escolher o melhor deles segundo algum critério como minimização do erro quadrático médio preditivo.

Como a metodologia estatística baseada no modelo de permutação aleatória ainda está em desenvolvimento, é de interesse obter preditores empíricos deduzidos sob este modelo e avaliar o desempenho tanto dos preditores quanto dos preditores empíricos com relação aos obtidos sob os outros modelos para populaçōes finitas ou infinitas. com ou sem erro na resposta. 
Na prática, a observação de covariáveis é freqüente. Para uma amostragem aleatória simples sem reposição, Li (2003) e Li e Stanek (2005) desenvolveram estimadores dos parâmetros populacionais (como a média ou o total) incorporando a informação de variáveis auxiliares, estendendo o método proposto por Stanek, Singer e Lencina (2004). Para isso, eles consideram um modelo de permutação aleatória simultánea da resposta e das variáveis auxiliares, numa forma semelhante ao modelo com regressões aparentemente não relacionadas (Seemingly Unrelated Regressions $S U R$ ). Os estimadores, obtidos como funções lineares da amostra, são não viciados e têm erro quadrático médio mínimo. No contexto de amostragem em dois estágios, é importante estender o modelo de permutação aleatória com o propósito de contemplar a inclusão de covariáveis, tanto para estimar algúm parâmetro populacional (como a média geral) quanto para predizer os parâmetros associados aos grupos amostrados.

\subsection{Proposta de pesquisa}

Este trabalho têm os seguintes objetivos:

1. Propor preditores empíricos do valor latente de grupos oriundos de populações finitas, baseados em amostragem com dois estágios.

2. Comparar os preditores ou preditores empíricos (tanto para variáveis contínuas quanto discretas com ou sem erro na resposta) obtidos sob os modelos para populações finitas com aqueles obtidos sob modelos baseados em populações infinitas.

3. Obter estimadores da resposta média ou total além de preditores do valor latente de grupos sob o modelo de permutação aleatória em situações em que há covariáveis presentes, quando a amostragem é feita em dois estágios.

Com relação ao primeiro objetivo, propomos estimadores para as variancias populacionais e construimos preditores empíricos substituindo as componentes de variancia pelos seus estimadores nas expressões das constantes de encolhimento. 
Com relação ao segundo objetivo, realizamos um estudo de simulação diferenciando dois casos: variáveis contínuas e variáveis discretas. Esses estudos são realizados em três etapas: 1) geração da população finita, 2) seleção das amostras em dois estágios sem reposição da população finita, e 3) cálculo dos preditores ou preditores empíricos e obtenção dos erros quadráticos médios preditivos. Para variáveis contínuas comparamo-los com os preditores desenvolvidos sob o modelo linear misto ou sob o modelo de Scott e Smith (1969). Para variáveis discretas comparamos os preditores obtidos sob o modelo linear misto generalizado com aqueles propostos para populações finitas.

Com relação ao terceiro objetivo, combinamos os enfoques propostos por $\mathrm{Li}$ (2003), Lencina (2002) e Stanek e Singer (2004) para incorporar a informação de variáveis auxiliares sob uma amostragem com dois estágios. 


\section{Capítulo 2}

\section{Preditores ótimos sob amostragem com dois estágios de populações finitas: variáveis contínuas}

\subsection{Introdução}

Usando um modelo de permutação aleatória induzido pelo planejamento amostral (para amostragem aleatória simples), Stanek, Singer e Lencina (2004) propuseram um método para obtenção de estimadores e preditores que utiliza as técnicas preditivas usualmente consideradas em modelos de superpopulação. O método foi estendido por Stanek e Singer (2004) para predizer os valores latentes associados a grupos sob uma amostragem balanceada em dois estágios considerando erro na. resposta.

Para uma amostragem em dois estágios balanceada de uma população finita com grupos de igual tamanho, em que a variável resposta é contínua e sob a suposição de que todas as componentes de variancia são conhecidas e as variâncias dentro de grupos são iguais. Stanek e Singer (2004) mostraram que os preditores dos valores latentes dos grupos realizados obtidos sob o modelo de permutação aleatória com crro na resposta tèm menor erro quadrático médio preditivo (EQM) que os obtidos sob o modelo linear misto (idealizado para populaşóes infinitas) e sob o modelo de Scott e Smith (1969) que se fundamenta em superpopulaşoes. Os cálculos foram 
realizados sob a suposição de que a estrutura de covariância verdadeira é aquela correspondente ao modelo de permutação aleatória. Para o caso em que as variâncias dentro dos grupos não são iguais, embora conhecidas, ainda não se têm resultados analíticos comparando os EQM dos preditores sob os diferentes modelos.

Na prática, as variâncias raramente são conhecidas e precisam ser estimadas, obtendo-se assim preditores empíricos. Neste capítulo vamos propor preditores empíricos do valor latente de grupos baseados tanto no modelo de permutação aleatória, quanto nos modelos de Scott e Smith (1969) e linear misto.

Como os preditores (empíricos) são obtidos sob cada modelo, na prática não assumimos uma estrutura de covariância comum e seria mais realista comparar os seus EQM nesta circunstância. Por outro lado, a obtenção dos EQM dos preditores empíricos é um processo complexo. Neste sentido, os artigos de Kackar e Harville (1984) e, mais recentemente, de Das, Jiang e Rao (2004) contêm aproximações do EQM dos preditores empíricos sob o modelo linear misto, com componentes de variância estimadas por máxima verossimilhança (ML) e máxima verossimilhança restrita (REML), enquanto que o trabalho de Prasad e Rao (1990) contêm aproximações do EQM para os preditores (ou estimadores) empíricos das médias de pequenas áreas obtidos sob três modelos diferentes (os desenvolvidos por Battese, Harter e Fuller (1988), Dempster, Rubin e Tsutakawa (1981) e Fay e Harriot (1979)), mas todas estas aproximações são obtidas sob a suposição de normalidade. Não temos conhecimento sobre resultados similares para preditores empíricos obtidos sob os modelos para populações finitas sem assumir normalidade. Com a finalidade de obter e comparar os EQM dos preditores empíricos sob os modelos linear misto, de Scott e Smith (1969) e de permutação aleatória desenvolvemos um estudo de simulação. Nesse estudo, também consideramos a comparação dos preditores obtidos sob a suposição que a estrutura de covariància particular de cada modelo é conhecida, como uma forma de ter um marco teórico de referència e poder avaliar também a perda pelo uso dos preditores empíricos. 


\subsection{Definições básicas}

Consideremos uma população finita composta por $N$ grupos com $M$ unidades cada um ( $N$ e $M$ conhecidos). Associada com cada unidade $t$ do grupo $s$ na população temos uma constante fixa $y_{s t}$, denominada parâmetro individual, $t=1, \ldots, M, s=1, \ldots, N$. Representamos o conjunto desses parâmetros por meio do vetor $\boldsymbol{y}=\left(\boldsymbol{y}_{1}^{\prime}, \ldots, \boldsymbol{y}_{N}^{\prime}\right)^{\prime}$, em que $\boldsymbol{y}_{s}=\left(y_{s 1}, \ldots, y_{s M}\right)^{\prime}$ corresponde ao vetor de parâmetros individuais associados às unidades do grupo $s$.

Definimos a média e a variância dos parâmetros individuais correspondentes às unidades do grupo $s, s=1, \ldots, N$, por $\mu_{s}=\frac{1}{M} \sum_{t=1}^{M} y_{s t}$ e $\sigma_{s}^{2}=\frac{1}{M-1} \sum_{t=1}^{M}\left(y_{s t}-\mu_{s}\right)^{2}$, respectivamente. Denotamos por $\sigma_{e}^{2}=\frac{1}{N} \sum_{s=1}^{N} \sigma_{s}^{2}$ a média das variâncias dentro dos grupos. Analogamente, definimos a média populacional e a variância entre grupos como $\mu=\frac{1}{N} \sum_{s=1}^{N} \mu_{s}$ e $\sigma^{2}=\frac{1}{N-1} \sum_{s=1}^{N}\left(\mu_{s}-\mu\right)^{2}$, respectivamente. Definimos também o coeficiente de correlação intra-classe dos grupos como $\rho_{s}=\frac{\sigma^{2}}{\sigma^{2}+\sigma_{e}^{2}}$.

Consideramos uma amostragem aleatória com dois estágios sem reposição dessa população. No primeiro estágio escolhe-se uma amostra de $n$ grupos e no segundo estágio escolhe-se uma amostra de $m$ unidades em cada um dos grupos anteriormente selecionados.

\subsection{Modelos para populações finitas}

\subsubsection{O modelo de permutação aleatória (MPA)}

Cada parâmetro individual da população finita pode se decompor na soma de três termos: a média populacional, o desvio da média do grupo $s$ com relação à média geral e o desvio do parâmetro individual com relação à média do grupo $s$, obtendo-se assim o seguinte modelo não estocástico:

$$
\begin{aligned}
y_{s t} & =\mu+\left(\mu_{s}-\mu\right)+\left(y_{s t}-\mu_{s}\right) \\
& =\mu+\beta_{s}+\epsilon_{s t}
\end{aligned}
$$

Podemos expressar matricialmente essa relação como $y=\mathrm{X} \mu+\mathrm{Z} \boldsymbol{\beta}+\epsilon$. en que $\mathrm{X}=1_{N M} \cdot \mathbf{Z}=\mathrm{I}_{N} \& 1_{M} \cdot \boldsymbol{\beta}=\left(\beta_{1}, \ldots, \beta_{N}\right)^{\prime} \mathrm{e} \boldsymbol{\epsilon}=\left(\epsilon_{11} \ldots, \epsilon_{N M}\right)^{\prime}$. Aqui, $1_{a}$ denota 
um vetor columa de dimensão $a \times 1$ com todos seus elementos iguais a 1 e $\mathbf{I}_{a}$, a matriz identidade de ordem $a$.

No modelo de permutação aleatória, a população finita $y$ é considerada uma realização de uma variável aleatória $Y$ induzida pelo esquema amostral em dois estágios. Os valores de $Y$ estão associados às possíveis permutações dos grupos e das unidades dentro dos grupos. Denotemos por $U$ e $U^{(s)}$ as duas matrizes de variáveis indicadoras (aleatórias) correspondentes às permutações dos grupos e das unidades dentro dos grupos, respectivamente, isto é,

$$
U=\left(\begin{array}{ccc}
U_{11} & \cdots & U_{1 N} \\
\vdots & \ddots & \vdots \\
U_{N 1} & \cdots & U_{N N}
\end{array}\right) \quad \text { e } U^{(s)}\left(\begin{array}{ccc}
U_{11}^{(s)} & \cdots & U_{1 M}^{(s)} \\
\vdots & \ddots & \vdots \\
U_{M 1}^{(s)} & \cdots & U_{M M}^{(s)}
\end{array}\right)
$$

com

$$
U_{i s}= \begin{cases}1 & \text { se o grupo } s \text { aparece no } i \text {-ésimo lugar na permutação aleatória } \\ & \text { dos grupos } \\ 0 & \text { em caso contrário }\end{cases}
$$

$$
U_{j t}^{(s)}= \begin{cases}1 & \text { se a unidade } t \text { aparece no } j \text {-ésimo lugar na permutação aleatória } \\ & \text { das unidades do grupo } s \\ 0 & \text { em caso contrário }\end{cases}
$$

para $i, s=1, \ldots, N$ e $j, t=1, \ldots, M$. Logo,

$$
\begin{aligned}
Y & =\left(U \otimes \mathrm{I}_{M}\right)\left(\underset{s=1}{\stackrel{N}{\oplus}} \boldsymbol{U}^{(s)}\right) \boldsymbol{y} \\
& =\left(\boldsymbol{U} \otimes \mathrm{I}_{M}\right)\left(\underset{s=1}{\stackrel{N}{\oplus}} U^{(s)}\right)(\mathrm{X} \mu+\mathrm{Z} \beta+\epsilon),
\end{aligned}
$$

em que $\otimes$ denota o produto Kronecker e $\oplus_{s=1}^{N} A_{s}$ denota uma matriz diagonal em blocos com blocos $\boldsymbol{A}_{s}$ (Searle, Casella e . McCulloch. 1992). Aplicando propriedades elementares de variáveis indicadoras e tendo em vista a estrutura simples de X e Z obtemos

$$
\begin{aligned}
Y & =\mathrm{X}_{\mu}+\mathrm{Z} U \boldsymbol{\beta}+\left(U \otimes \mathrm{I}_{M}\right)\left(\underset{s=1}{\left.\stackrel{N}{\oplus} U^{(s)}\right) \epsilon}\right. \\
& =\mathrm{X}_{\mu}+\mathrm{Z} B+E .
\end{aligned}
$$


Note que, dada a aleatoriedade introduzida por $\boldsymbol{U}$, as componentes de $\boldsymbol{B}=\boldsymbol{U} \boldsymbol{\beta}$, dadas por $B_{i}=\sum_{s=1}^{N} U_{i s} \beta_{s}$, para $i=1, \ldots, N$, são efeitos aleatórios que representam os desvios do valor latente do grupo correspondente à $i$-ésima posição da permutação aleatória com relação à média populacional. Chamamos (2.1) de modelo misto de permutação aleatória.

De (2.1) segue que

$$
E_{\xi_{1} \xi_{2}}(Y)=\mathrm{X} \mu
$$

$\mathrm{e}$

$$
\operatorname{Var}_{\xi_{1} \xi_{2}}(\boldsymbol{Y})=\sigma_{e}^{2}\left(\mathbf{I}_{N} \otimes \mathbf{I}_{M}\right)+\sigma^{* 2}\left(\mathbf{I}_{N} \otimes \mathbf{J}_{M}\right)-\frac{\sigma^{2}}{N}\left(\mathbf{J}_{N} \otimes \mathbf{J}_{M}\right)
$$

em que $\sigma^{* 2}=\sigma^{2}-\frac{\sigma_{e}^{2}}{M}, \mathbf{J}_{a}=\mathbf{1}_{a} \mathbf{1}_{a}^{\prime}$ e os sub-índices $\xi_{1}$ e $\xi_{2}$ denotam a esperança com relação à permutação dos grupos e das unidades, respectivamente.

Às vezes, os parâmetros individuais não podem ser observados diretamente, mas com um certo erro aleatório. Nesse caso, o valor observado pode ser escrito como $\boldsymbol{y}+\boldsymbol{W}$, sendo $\boldsymbol{W}=\left(W_{\text {st }}\right) \in \mathbb{R}^{N M} \operatorname{com} E\left(W_{s t}\right)=0$ e $\operatorname{Var}\left(W_{s t}\right)=\sigma_{s t}^{2}$. Obtemos assim um modelo misto de permutação aleatória com erro na resposta, representado por

$$
\begin{aligned}
& Y^{*}=\left(U \otimes \mathrm{I}_{M}\right)\left(\bigoplus_{s=1}^{N} U^{(s)}\right)(y+W) \\
& =\mathrm{X} \mu+\mathrm{Z} B+E+\left(U \otimes \mathrm{I}_{M}\right)\left(\underset{s=1}{\stackrel{N}{\oplus} U^{(s)}}\right) W \\
& =\mathrm{X} \mu+\mathrm{Z} B+E+W^{*} \text {. } \\
& =Y+W^{*}
\end{aligned}
$$

Neste caso,

$$
E_{\xi_{1} \xi_{2} \xi_{3}}\left(Y^{*}\right)=\mathrm{X} \mu
$$

e

$$
\operatorname{Var}_{\xi_{1} \xi_{2} \xi_{3}}\left(\boldsymbol{Y}^{*}\right)=\left(\sigma_{e}^{2}+\sigma_{r}^{2}\right)\left(\mathbf{I}_{N} \otimes \mathbf{I}_{M}\right)+\sigma^{* 2}\left(\mathbf{I}_{N} \otimes \mathbf{J}_{M}\right)-\frac{\sigma^{2}}{N}\left(\mathbf{J}_{N} \otimes \mathbf{J}_{. M}\right)
$$

em que o sub-índice $\xi_{3}$ denota a esperança com relação à distribuição do erro na resposta e $\sigma_{r}^{2}=\frac{1}{N . M} \sum_{s=1}^{N} \sum_{t=1}^{M} \sigma_{s t}^{2}$.

Em ambos os modelos (com e sem erro na resposta) a estrutura de covariância da variável aleatória $Y\left(Y^{*}\right)$ é induzida pelo esquema amostral e nenhuma suposição 
distribucional é considerada. Isto faz com que o modelo possa ser aplicado a uma. ampla gama de situações práticas.

Para os elementos amostrais $Y_{S}^{*}=Y_{S}+W_{S}^{*}$, os momentos induzidos pelo modelo são

$$
\begin{gathered}
E_{\xi_{1} \xi_{2} \xi_{3}}\left(Y_{S}^{*}\right)=\mathbf{X}_{S} \mu=1_{n m} \mu \\
\operatorname{Var}_{\xi_{1} \xi_{2} \xi_{3}}\left(Y_{S}^{*}\right)=\left(\sigma_{e}^{2}+\sigma_{r}^{2}\right)\left(\mathbf{I}_{n} \otimes \mathbf{I}_{m}\right)+\sigma^{* 2}\left(\mathbf{I}_{n} \otimes \mathbf{J}_{m}\right)-\frac{\sigma^{2}}{N}\left(\mathbf{J}_{n} \otimes \mathbf{J}_{m}\right)
\end{gathered}
$$

Note que a variância intra-grupos, $\sigma_{e}^{2}$, é a mesma para todos os grupos e tanto as unidades dentro do mesmo grupo quanto as de diferentes grupos são correlacionadas (neste último caso, negativamente).

\subsubsection{O modelo de Scott e Smith (MSS)}

Scott e Smith (1969) definem um modelo de superpopulação em que a população finita $y$ é considerada uma realização de um vetor de variáveis aleatórias $Y$ tal que

$$
E(\boldsymbol{Y})=\mathbf{X} \mu \quad \text { e } \quad \operatorname{Var}(\boldsymbol{Y})=\oplus_{i=1}^{N}\left(\sigma_{i}^{2} \mathbf{I}_{M}+\sigma^{2} \mathbf{J}_{M}\right)
$$

sendo $\mathrm{X}=1_{N M}$. Note que, sob este modelo, o vetor $Y$ não está diretamente ligado à população de unidades como no modelo de permutação aleatória. Neste contexto, as médias dos grupos $\mu_{1}, \ldots, \mu_{N}$ podem ser consideradas como realizações de variáveis aleatórias independentes e identicamente distribuídas $\Lambda_{1}, \ldots, \Lambda_{N}$ tal que, para cada $i=1, \ldots, N, E\left(\Lambda_{i}\right)=\mu$ e $\operatorname{Var}\left(\Lambda_{i}\right)=\sigma^{2}$. Assim, $\sigma^{2}$ pode ser interpretada como a variância da distribuição das variáveis aleatórias $\Lambda_{1}, \ldots, \Lambda_{N}$, das quais $\mu_{1}, \ldots, \mu_{N}$ constituem uma amostra. Sob este modelo, os elementos dentro do mesmo grupo são correlacionados, mas os elementos em diferentes grupos, não.

Este modelo foi estendido por Bolfarine e Zacks (1992) para incluir erros na resposta. Nesse caso, o valor observado $\boldsymbol{y}+\boldsymbol{W}$ é considerado una realização da variável aleatória $Y^{*}=Y+W$, com $\mathrm{W} \sim N\left(\mathbf{0}, \underset{i=1}{N} \sigma_{r}^{2} \mathrm{I}_{M}\right)$ independente de $Y$. Logo.

$$
\left.E\left(Y^{*}\right)=\mathrm{X}_{\mu} \quad \text { e } \quad \operatorname{Var}\left(\boldsymbol{Y}^{*}\right)=\oint_{i=1}^{N}\left(\left(\sigma_{i}^{2}+\sigma_{r}^{2}\right) \mathrm{I}_{M}+\sigma^{2} \mathrm{~J}_{M}\right)\right)
$$

Para os elementos amostrais $Y_{S}^{*}$, os momentos induzidos pelo modelo são como em (2.3), com $n$ e $m$ no lugar de $N$ e $M$, respectivamente. 


\subsection{O modelo linear misto $(M L M)$ para populações infinitas}

Apesar de não incorporar a estrutura finita da população, modelos para populações infinitas são muitas vezes usados na prática. Searle e Fawcett (1970) desenvolveram uma regra para converter as esperanças dos quadrados médios obtidos sob modelos de componentes de variância para populações infinitas em esperanças obtidas sob modelos para populações finitas. No entanto, essas regras têm sido pouco usadas devido, em parte, à falta de desenvolvimentos teóricos posteriores e a uma carência de software que as implemente.

Sob o modelo linear misto, considera-se que os dados da amostragem com dois estágios foram escolhidos de uma população conceitualmente infinita (interpretada como limite da população finita de interesse). Neste caso, o vetor (amostral) de respostas, $Y_{S}=\left(Y_{11}, \ldots, Y_{n m}\right)^{\prime}$, em que $Y_{i j}$ corresponde a $j$-ésima resposta no $i$ ésimo grupo, $i=1, \ldots, n, j=1, \ldots, m$, pode ser modelado como

$$
Y_{S}=\mathrm{X}_{S} \mu+\mathrm{Z}_{S} \boldsymbol{B}_{S}+\boldsymbol{E}_{S}
$$

em que $\mathrm{X}_{S}=1_{n} \otimes 1_{m}, \mathbf{Z}_{S}=\mathrm{I}_{n} \otimes 1_{m}, B_{S}=\left(B_{1}, \ldots, B_{n}\right)^{\prime} \operatorname{com} B_{S} \sim N\left(0, \sigma^{2} \mathbf{I}_{n}\right)$, $\boldsymbol{E}_{S}=\left(\boldsymbol{E}_{S 1}^{\prime}, \ldots, \boldsymbol{E}_{S n}^{\prime}\right)^{\prime} \operatorname{com} \boldsymbol{E}_{S i} \sim N\left(0, \sigma_{i}^{2} \mathbf{I}_{m}\right)$ e $\boldsymbol{B}_{S}$ e $\boldsymbol{E}_{S}$ independentes. Aqui, $B_{i}$ representa o efeito aleatório que corresponde ao desvio da resposta média esperada das unidades do grupo $i$ com relação à média geral $\mu\left(\mu=E\left(Y_{i j}\right)\right)$ e $E_{i j}$ é o desvio aleatório da resposta esperada (condicional) da unidade $j$ com relação à resposta média esperada (condicional) das unidades do grupo $i$. Então

$$
Y_{S} \sim N\left(\mathbf{X}_{S} \mu, \underset{i=1}{\oplus}\left(\sigma_{i}^{2} \mathbf{I}_{m}+\sigma^{2} \mathbf{J}_{m}\right)\right) .
$$

Se em (2.4) incluirmos aditivamente um outro termo $W_{S} \sim N\left(0, \sigma_{r}^{2} \mathbf{I}_{n m}\right)$ independente de $B_{S}$ e $\boldsymbol{E}_{S}$, correspondente ao erro na resposta, segue que

$$
\boldsymbol{Y}_{S}^{*}=\boldsymbol{Y}_{S}+\boldsymbol{W}_{S} \sim N\left(\mathbf{X}_{S} \mu \cdot \underset{i=1}{\stackrel{N}{\ominus}}\left(\left(\sigma_{i}^{2}+\sigma_{r}^{2}\right) \mathbf{I}_{m}+\sigma^{2} \mathbf{J}_{m}\right)\right) .
$$

Sob este modelo. a variância dos efeitos aleatórios $\sigma^{2}$ pode ser interpretada como a variancia da variável aleatória $\mu+B_{i}$ que conceitualmente assume um número infinito de valores. Além disso, as unidades do mesmo grupo são corrclacionados, mas as unidades em diferentes grupos, não. 


\subsection{Preditores de características populacionais}

\subsubsection{Definição dos preditores}

Nosso principal interesse é predizer o valor latente do $i$-ésimo grupo realizado, $i=1, \ldots, n$, i.e., as respostas médias esperadas para os grupos selecionados na. amostragem. Para o grupo $i$, com $i \leq n$, podemos representar esse valor como $T_{i}=g^{\prime} Y$, em que $g=\frac{1}{M} \boldsymbol{e}_{i} \otimes 1_{M}$ e $\boldsymbol{e}_{i}$ denota um vetor coluna de dimensão $N$, com 1 na posição $i$ e zero nas restantes. A partir dos valores amostrais $Y_{S}^{*}=\left(Y_{i j}^{*}\right)=$ $\left(\boldsymbol{Y}_{S 1}^{*}, \boldsymbol{Y}_{S 2}^{*}{ }^{\prime}, \ldots, \boldsymbol{Y}_{S n}^{*}{ }^{\prime}\right)^{\prime}, \operatorname{com} \boldsymbol{Y}_{S i}^{*}=\left(Y_{i 1}^{*}, Y_{i 2}^{*}, \ldots Y_{i m}^{*}\right)^{\prime}$, os preditores de $T_{i}(i \leq n)$ sob os modelos (2.3) e (2.5) podem ser obtidos como

$$
\widehat{T}_{i}=\widehat{\mu}+k_{i}^{(\text {modelo })}\left(\bar{Y}_{i}^{*}-\widehat{\mu}\right)
$$

em que $\widehat{\mu}=\frac{\sum_{i=1}^{n} \bar{Y}_{i}^{*} /\left(m \sigma^{2}+\sigma_{i}^{2}+\sigma_{r}^{2}\right)}{\sum_{i=1}^{n} 1 /\left(m \sigma^{2}+\sigma_{i}^{2}+\sigma_{r}^{2}\right)}$ e $\bar{Y}_{i}^{*}=\frac{1}{m} \sum_{j=1}^{m} Y_{i j}^{*}$, e as constantes de encolhimento $k_{i}^{(\text {modelo })}$ são

$$
k_{i}^{(L M)}=\frac{m \sigma^{2}}{m \sigma^{2}+\sigma_{i}^{2}+\sigma_{r}^{2}}, \quad \quad \text { para o } M L M,
$$

e

$$
k_{i}^{(S S)}=k_{i}^{(L M)}+f\left(1-k_{i}^{(L M)}\right)=\frac{m \sigma^{2}+f\left(\sigma_{i}^{2}+\sigma_{r}^{2}\right)}{m \sigma^{2}+\sigma_{i}^{2}+\sigma_{r}^{2}}, \quad \text { para o } M S S
$$

enquanto que para o modelo (2.2),

$$
\widehat{T}_{i}=\bar{Y}^{*}+k^{(P A)}\left(\bar{Y}_{i}^{*}-\bar{Y}^{*}\right)
$$

em que $\bar{Y}^{*}=\frac{1}{n m} \sum_{i=1}^{n} \sum_{j=1}^{m} Y_{i j}^{*}$, e a constante de encolhimento é

$$
k^{(P A 1)}=\frac{m \sigma^{2}}{m \sigma^{* 2}+\sigma_{e}^{2}+\sigma_{r}^{2}}=\frac{m \sigma^{2}}{m \sigma^{2}+(1-f) \sigma_{e}^{2}+\sigma_{r}^{2}} .
$$

$\operatorname{com} f=\frac{m}{11}$.

Essas expresões combinam as respostas das unidades observadas com preditores da resposta das não observadas, mas diferem em relação às constantes de encolhimento usadas. Estas constantes de encolhimento são funções das componentes de variancia populacionais e da quantidade de unidades amostradas; sob os modelos 
para populações finitas também dependem dos tamanhos dos grupos. Estas constantes de encolhimento satisfazem que $0 \leq k_{i}^{(L M)} \leq k_{i}^{(S S)} \leq 1$ e $0 \leq k^{(P . A)} \leq 1$ (ver seção A.1 no apêndice A).

Quando as variâncias intra-grupo são idènticas (e iguais a $\sigma_{e}^{2}$ ), os preditores de $T_{i}$, para $i \leq n$, sob os três modelos podem ser obtidos como

$$
\widehat{T}_{i}=\bar{Y}^{*}+k^{(\text {mollelo })}\left(\bar{Y}_{i}^{*}-\bar{Y}^{*}\right)
$$

em que

$$
\begin{array}{ll}
k^{(L M)}=\frac{m \sigma^{2}}{m \sigma^{2}+\sigma_{e}^{2}+\sigma_{r}^{2}}, & \text { para o MLM, } \\
k^{(S S)}=k^{(L M)}+f\left(1-k^{(L M)}\right)=\frac{m \sigma^{2}+f\left(\sigma_{e}^{2}+\sigma_{r}^{2}\right)}{m \sigma^{2}+\sigma_{e}^{2}+\sigma_{r}^{2}}, & \text { para o } M S S,
\end{array}
$$

$$
k^{(P A)}=\frac{m \sigma^{2}}{m \sigma^{* 2}+\sigma_{e}^{2}+\sigma_{r}^{2}}=\frac{m \sigma^{2}}{m \sigma^{2}+(1-f) \sigma_{e}^{2}+\sigma_{r}^{2}}, \quad \text { para o } M P A .
$$

Neste caso, as constantes de encolhimento satisfazem que $0 \leq k^{(L M)} \leq k^{(P A)} \leq$ $k^{(S S)} \leq 1$ (ver seção A.1 no apêndice A).

\subsubsection{Comparação dos preditores}

A comparação dos preditores obtidos sob os diferentes modelos visa encontrar aquele mais próximo à realidade ou, equivalentemente, achar aquele modelo que a representa melhor. Os três modelos aqui considerados dependem de diferentes suposições; o MPA é baseado no planejamento e só assume a existência dos momentos de primcira e segunda ordem. É sob estas suposiçōes menos limitadas que Stanek e Singer (2004) mostraram que o preditor obtido sob o MPA apresenta o mínimo EQM, independentemente do número e tamanho dos grupos ou dos coeficientes de correlação intra-classe dos grupos $\left(\rho_{s}\right)$ e unidades $\left(\rho_{t}\right)^{1}$. De fato. as comparações foram realizadas sob a hipótese de que a estrutura de covariância das variáveis aleatórias é aquela correspondente ao MPA. Em certas circunstancias, os preditores

\footnotetext{
${ }^{1} \mathrm{O}$ coeficiente de correlação intra-classe das unidades é definido como $\rho_{t}=\frac{\sigma_{t}^{2}}{\sigma_{t}^{2}+\sigma_{t}^{2}}$. Fun particular. $\rho_{t}=1$ corresponde à situaģ̄o sem erro na resposta.
} 
obtidos sob os diferentes modelos podem ser bastante semelhantes (i.e., podemos ter EQM muito próximos) e qualquer um deles pode ser considerado como uma representação adequada da realidade, enquanto que em outras, só algum deles é o mais apropriado. Nosso interesse é identificar estas situações, em especial quando consideramos preditores empíricos.

Como um primeiro passo no estudo das semelhanças e diferenças entre os preditores, estudamos o comportamento das constantes de encolhimento teóricas quando as variâncias intra-grupos são iguais, embora elas não levem em conta a fração de amostragem dos grupos como no caso do EQM. Na Figura 2.1 comparamos esse comportamento para diferentes valores de $f, \rho_{s}$ e $\rho_{t}$; incluimos também a média do grupo $(M G)$. A linha "sólida" serve de referência para comparar os preditores, pois ela corresponde ao preditor obtido sob o MPA.

Esperamos encontrar pequenas diferenças entre os preditores, quase independentemente de $f$, quando ambos $\rho_{s}$ e $\rho_{t}$ tendem para 1. A partir da Figura 2.1, também observamos que quando $f$ tende para zero, $k^{(L M)}, k^{(S S)}$ e $k^{(P A)}$ são muito semelhantes (e todas são diferentes de $k^{(M G)}=1$ ). Quando não temos erro na resposta $\left(\rho_{t}=1\right)$ e amostramos todas as unidades do grupo $(f=1)$, temos $k^{(P A)}=k^{(S S)}=k^{(M G)}=1$ (e todas são diferentes de $k^{(L M)}$ ), de modo que o melhor preditor é a média do grupo. Quando $\rho_{t}$ se aproxima de zero, $k^{(L M)}$ aproxima-se de $k^{(P A)}$ (quase independentemente de $f$ ), de modo que esperamos que nesse contexto os preditores obtidos sob estes dois modelos tenham um comportamento similar.

Quando ambos os $\rho_{s}$ e $\rho_{t}$ tendem para zero, a $M G$ apresenta um desempenho ruim com relação ao preditor obtido sob o MPA à medida que $f$ se aproxima de zero, enquanto que o preditor obtido sob o $M S S$ apresenta este comportamento (ruim) quando $f$ se aproxima de um. Além disso, esperamos que o preditor obtido sob o $M L M$ mostre este desempenho ruim quando $\rho_{s}$ tende para zero e ambos $\rho_{t}$ e $f$ tendem para um.

Para completar o estudo do desempenho dos diferentes preditores comparamos os respectivos EQM. Como estes EQM dependem tanto das características da população quanto da amostragem, vamos adiar seu tratamento para a seção 2.8. em conjunção com o estudo de simulaçào. 


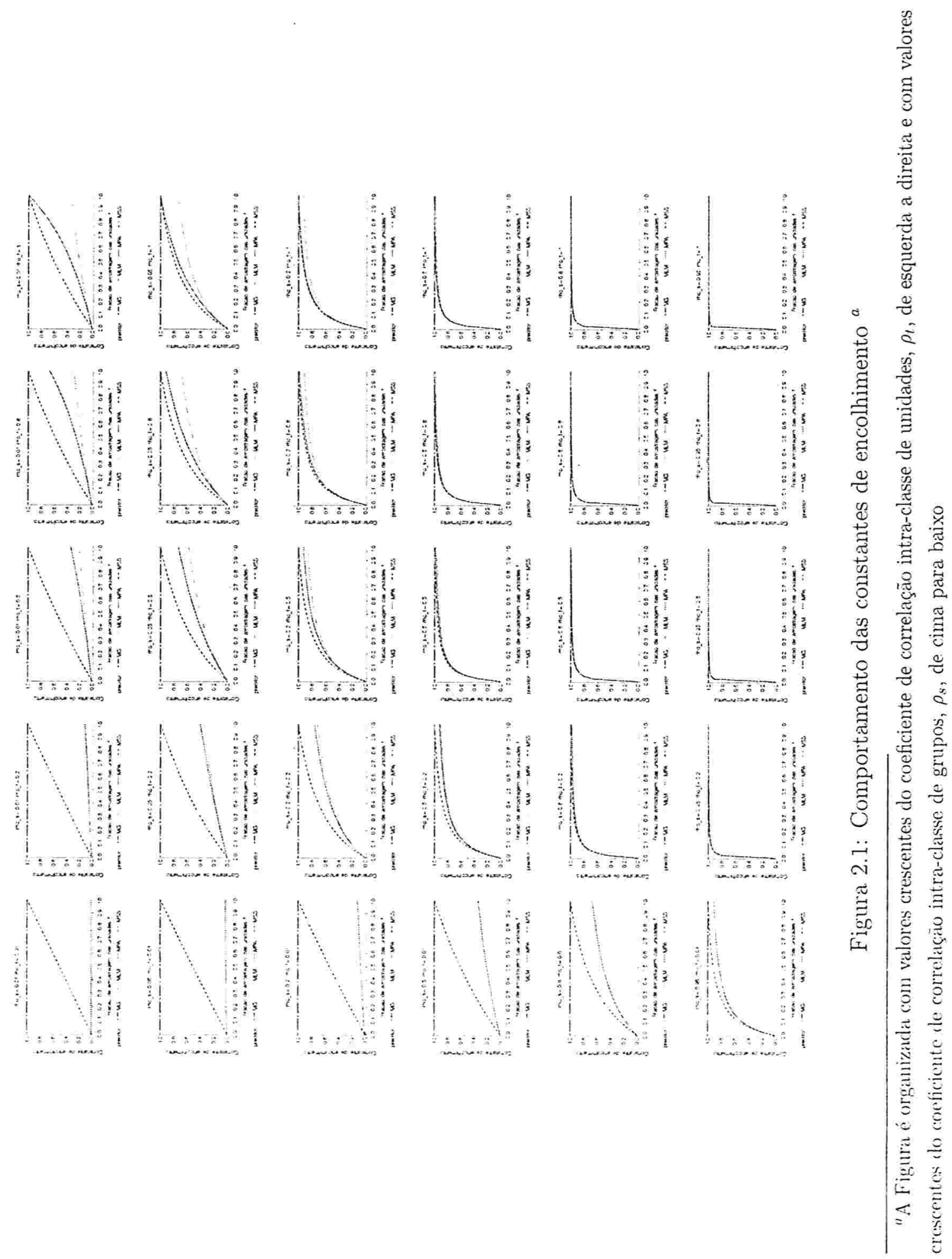




\subsection{Preditores empíricos}

Na prática, as componentes de variância são geralmente desconhecidas e precisamos estimá-las para obter estimadores das constantes de encolhimento. Os preditores empíricos são obtidos substituindo as constantes de encolhimento pelos seus estimadores. Como os modelos para populações finitas aqui usa.dos não necessitam de suposições sobre a distribuição da resposta além dos momentos de primeira e segunda ordem, vamos estimar as componentes de variância pelo método dos momentos a partir das esperanças dos quadrados médios da. análise de variância (ver detalles na seção A.2 do Apêndice A).

Podemos assim obter os seguintes estimadores, pelo método dos momentos, para as constantes de encolhimento:

$\checkmark M P A$

$$
\widehat{k}_{1}^{(P A)}= \begin{cases}0 & \text { se } Q M E=0 \\ \max \left(0, \frac{Q M E-(1-f) Q M D-f \sigma_{r}^{2}}{Q M E}\right) & \text { se } Q M E>0\end{cases}
$$

ou

$$
\widehat{k}_{2}^{(P A)}= \begin{cases}0 & \text { se } Q M E=0 \\ \max \left(0, \frac{Q M E-\left(1-f \rho_{t}\right) Q M D}{Q M E}\right) & \text { se } Q M E>0\end{cases}
$$

em que $Q M E$ e $Q M D$ denotam os quadrados médios entre e dentro de grupos, respectivamente.

$\checkmark M L M$ - Variâncias intra-grupos iguais

$$
\widehat{k}^{(L M)}= \begin{cases}0 & \text { se } Q M E=0 \\ \max \left(0 . \frac{Q M E-Q M D}{Q M E}\right) & \text { se } Q M E>0\end{cases}
$$

$\checkmark M S S$ - Variâncias intra-grupos iguais

$$
\widehat{k}_{1}^{(S S)}= \begin{cases}f & \text { se } 0 \leq Q . M E \leq Q M D \\ \max \left(0, \frac{Q M E-(1-f) Q M D}{Q . M E}\right) & \text { se } Q M E>Q M D\end{cases}
$$


oul

$$
\widehat{k}_{2}^{(S S)} \begin{cases}f & \text { se } Q M E=0 \\ \max \left(0, \frac{Q M E-(1-f) Q M D}{Q M E}\right) & \text { se } Q M E>0\end{cases}
$$

$\checkmark M L M$ - Variâncias intra-grupos diferentes

$$
\widehat{k}_{i 1}^{(L M)}= \begin{cases}0 & \text { se } c_{i}=0 \\ \max \left(0, \frac{Q M E-Q M D}{c_{i}}\right) & \text { em caso contrário }\end{cases}
$$

ou

$$
\widehat{k}_{i 2}^{(L M)}= \begin{cases}0 & \text { se } d_{i}^{2}=0 \\ \min \left[\max \left(0, \frac{Q M E-Q M D}{d_{i}^{2}}\right), 1\right] & \text { em caso contrário }\end{cases}
$$

em que $c_{i}=S_{i}^{2}+\max (0, Q M E-Q M D), S_{i}^{2}=\frac{1}{m-1} \sum_{j=1}^{m}\left(Y_{i j}^{*}-\bar{Y}_{i}^{*}\right)^{2} \mathrm{e}$ $d_{i}^{2}=\frac{n m\left(\bar{Y}_{i}^{*}-\bar{Y}^{*}\right)^{2}}{n-1}$.

\section{$\checkmark M S S$ - Variâncias intra-grupos diferentes}

$$
\widehat{k}_{i l}^{(S S)}=\widehat{k}_{i l}^{(L M)}+f\left(1-\widehat{k}_{i l}^{(L M)}\right) \quad \text { para } l=1,2
$$

oul

$$
\widehat{k}_{i 3}^{(S S)}= \begin{cases}0 & \text { se } d_{i}^{2}=0 \\ \min \left[\frac{\max (0, Q M E-Q M D)+f S_{i}^{2}}{d_{i}^{2}}, 1\right] & \text { em caso contrário }\end{cases}
$$

Para obter os preditores empíricos sob o MSS e o MLM, também precisamos incorporar os estimadores das componentes de variância em

$$
\widehat{\mu}=\frac{\sum_{i=1}^{n} \bar{Y}_{i}^{*} /\left(m \sigma^{2}+\sigma_{i}^{2}+\sigma_{r}^{2}\right)}{\sum_{i=1}^{n} 1 /\left(m \sigma^{2}+\sigma_{i}^{2}+\sigma_{r}^{2}\right)} .
$$

No caso de variàncias intra-grupos iguais, $\widehat{\mu}=\bar{Y}^{*}$. que é combinada com as respectivas constantes $\widehat{k}$ para obter os preditores empíricos. No caso de variảncias intra-grupos diferentes. propomos

$$
\widehat{\mu}_{1}=\frac{\sum_{i=1}^{n} \bar{Y}_{i}^{*} / c_{i}}{\sum_{i=1}^{n} 1 / c_{i}}
$$


e

$$
\widehat{\mu}_{2}=\frac{\sum_{i=1}^{n} \bar{Y}_{i}^{*} / d_{i}^{2}}{\sum_{i=1}^{n} 1 / d_{i}^{2}}=\frac{\sum_{i=1}^{n} \bar{Y}_{i}^{*} /\left(\bar{Y}_{i}^{*}-\bar{Y}^{*}\right)^{2}}{\sum_{i=1}^{n} 1 /\left(\bar{Y}_{i}^{*}-\bar{Y}^{*}\right)^{2}}
$$

O primeiro é combinado com $\widehat{k}_{i 1}^{(L M)}$ e $\widehat{k}_{i 1}^{(S S)}$, enquanto que o segundo é combinado com $\widehat{k}_{i 2}^{(L M)}, \widehat{k}_{i 2}^{(S S)}$ e $\widehat{k}_{i 3}^{(S S)}$ para obter os preditores empíricos.

Para comparar o desempenho destes preditores empíricos, desenvolvemos um estudo por simulação.

\subsection{Estudo por simulação}

Para comparar os EQM dos diferentes preditores e preditores empíricos de valores latentes de grupos provenientes de uma população finita balanceada obtidos por intermédio de amostragem em dois estágios realizou-se um estudo por simulação. $\mathrm{O}$ estudo consistiu de três etapas: 1) a geração da população finita com agrupamentos, 2) a seleção das amostras em dois estágios a partir da população com agrupamentos e 3) o cálculo dos preditores e preditores empíricos e seus respectivos EQM.

\subsubsection{Geração da população finita}

Para ser bastante abrangentes, consideramos populações finitas diferindo com relação: 1) à quantidade $(N)$ e ao tamanho $(M)$ dos grupos, 2) à forma da distribuição da variável resposta, 3) às variâncias entre e dentro de grupos, $\sigma^{2}$ e $\sigma_{e}^{2}$, e 4) à suposição de variâncias intra-grupos iguais ou diferentes. A presença ou não de erro na resposta é considerada na etapa de amostragem.

Geramos cada população finita de unidades e grupos usando os percentis de uma das seguintes distribuições: normal, uniforme, beta e gama. Usamos a mesma distribuição para gerar os efeitos associados às unidades para todos os grupos em cada população. Para os parâmetros associados aos grupos, consideramos tanto situaçōes com distribuições iguais quanto diferentes daquelas usadas para as midades.

Para cada simulação, a população consistiu de $N$ grupos com $M$ unidades por grupo. Representamos o paràmetro associado ao grupo $s$ por $\mu_{s}$ e a média desses paràmetros por $\mu$. Uma vez fixada a variancia entre grupos, $\sigma^{2}$, dividimos o intervalo [0. 1] em $N+1$ intervalos de ignal tamanho e obtivemos os percentis correspondentes 
ao limite superior de cada intervalo a partir da distribuição de probabilidade escolhida. Redefinimos os parâmetros dos grupos centrando-os em $\mu$ e transformando-os de forma que sua variância coincidisse com $\sigma^{2}=\frac{1}{N-1} \sum_{s=1}^{N}\left(\mu_{s}-\mu\right)^{2}$.

A seguir, geramos os efeitos associados às $M$ unidades de cada grupo usando os percentis da distribuição selecionada e forçamos a que estes efeitos tivessem média zero. Consideramos casos em que a variância dos efeitos das unidades era constante ou variava proporcionalmente à $\sqrt{\max \left(0,1 ; \mu_{s} / \mu\right)}$. Os parâmetros associados às unidades, representados por $y_{s t}$, foram construídos adicionando-se a média do grupo ao efeito da unidade. A variância dos parâmetros associados às unidades no grupo $s$ foi $\sigma_{s}^{2}=\frac{1}{M-1} \sum_{t=1}^{M I}\left(y_{s t}-\mu_{s}\right)^{2}$ e a média das variâncias intra-grupo foi $\sigma_{e}^{2}=\frac{1}{N} \sum_{s=1}^{N} \sigma_{s}^{2}$. Os efeitos das unidades foram transformados de forma que sua média fosse zero em cada grupo e a variância média fosse igual a $\sigma_{e}^{2}$.

Foi desenvolvida uma sub-rotina (PSGEN) no sistema SAS (disponível em www.umass.edu/cluster/ed) para gerar as populações finitas segundo os detalhes apresentados nesta seção. Nesta sub-rotina, o usuário pode especificar os parâmetros usados na simulação.

As características das populações simuladas estão resumidas na Tabela 2.1. Cento e doze populações correspondentes a todas as combinações das correlações intra-classe dos grupos e das unidades, considerando tanto variâncias intra-grupos iguais quanto diferentes foram geradas no caso 1 . Nos casos 2 e 3 , foram geradas 336 $(=6 \times 7 \times 8)$ populações, enquanto que nos casos 4 e 5 foram geradas $112(=2 \times 7 \times 8)$ populações. Um total de 1008 populações foi avaliado.

\subsubsection{Amostragem em dois estágios}

A partir de uma lista com os rótulos dos grupos, uma amostra aleatória simples sem reposição de $n$ rótulos foi obtida em cada população. A amostra de rótulos dos grupos foi então combinada com os dados populacionais e uma amostra aleatória simples de $m$ unidades foi escolhida sem reposição a partir de cada um dos grupos previamente selecionados. Nos casos com erro na resposta. ele foi acrescentado aos parâmetros das unidades $y_{s t}$ durante a seleção das amostras em dois estágios. Vamos nos referir a este processo como um ensaio.

Para cada população foram usadas très fraçōes de amostragem dos grupos. 


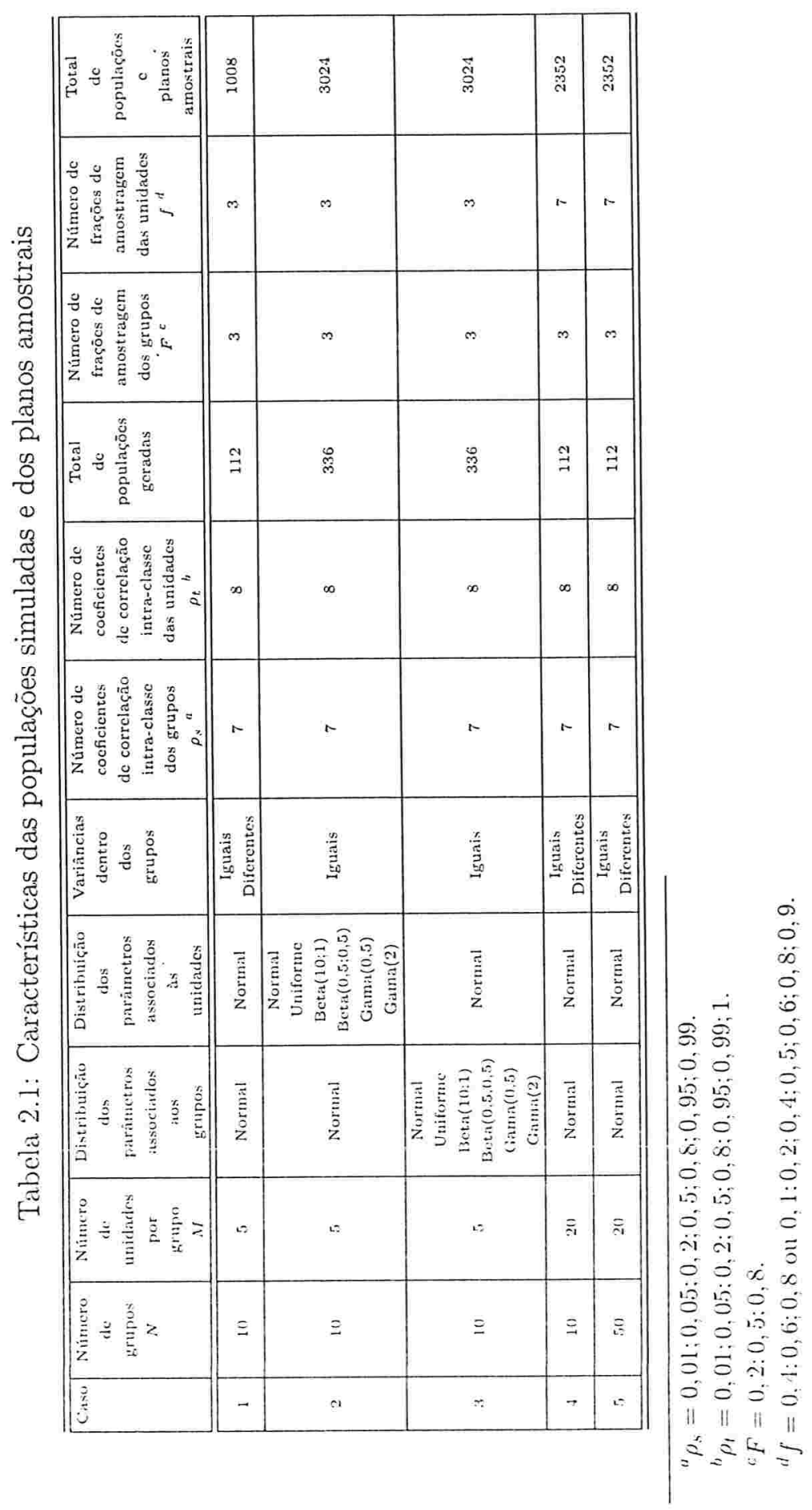


$F=\frac{n}{N^{\prime}}=0,2 ; 0,5$ e 0,8. Para cada população com $M=5$ (casos 1, 2 e 3 da Tabela 2.1 ), foram usadas três frações de amostragem das unidades, $f=\frac{m}{M}=0,4 ; 0,6$ e 0,8 . Como conseqüência, foram empregados nove planos amostrais para cada população gerada. Para as outras populações $(M=20)$, sete frações de amostragem das unidades, $f=0,1 ; 0,2 ; 0,4 ; 0,5 ; 0,6 ; 0,8$ e 0,9 , foram consideradas, dando como resultado 21 planos amostrais para cada população gerada. No total, 11760 populações/planos amostrais foram simulados. Para cada um deles foram usadas 10000 réplicas.

\subsubsection{Preditores}

Depois de obter as amostras em dois estágios, calculamos os preditores e preditores empíricos sob cada modelo, conforme indicado nas seções 2.5 e 2.6, respectivamente. Para o $M L M$ e o $M S S$ assumimos que os valores conhecidos de $\sigma^{2}$ e $\sigma_{e}^{2}$ correspondem às variâncias entre e dentro dos grupos.

Denotamos por EQMS o erro quadrático médio preditivo obtido por simulação sob as suposições de cada modelo particular para o caso de variâncias conhecidas e por EQME, aquele obtido por simulação para o caso de variâncias desconheciclas. Este último corresponde aos preditores empíricos especificados na seção 2.6.

Foram desenvolvidas duas sub-rotinas em SAS (disponíveis em www.umass.edu/cluster/ed) para selecionar as amostras em dois estágios, obter os preditores e preditores empíricos e calcular os EQM.

\subsection{Resultados da simulação}

Quando as variâncias intra-grupos são iguais e sob a hipótese de que a estrultura de covariància é aquela correspondente ao MPA, Stanek e Singer (2004) obtèm expressões para o EQM dos preditores obtidos sob o MLM. o MSS e o MPA. Esses autores apresentam um estudo de simulação em pequena escala para comparar o desempenho dos preditores. dependendo da fraşào de amostragem das unidades $(f)$ e das correlações intra-classe $\left(\rho_{s}\right.$ e $\left.\rho_{t}\right)$, assumindo $u$ modelo de permutação aleatória em dois estágios. Aqui complementamos o estudo de simulação incorporando a fração de amostragem dos grupos e calculando tanto os preditores quanto 
os preditores empíricos sob as suposições de cada modelo particular (para variâncias intra-grupos iguais ou diferentes), avaliando também o desempenho da média do grupo.

Primeiramente, comparamos o EQMS dos preditores obtidos sob cada modelo para fornecer um marco teórico contra o qual comparar os resultados referentes aos preditores empíricos. A seguir, avaliamos a perda relativa (em termos do EQME com relação ao EQMS) que ocorre quando substituímos a constante de encolhimento teórica pela sua estimativa, isto é, quando usamos o preditor empírico. Finalmente, avaliamos o desempentio dos preditores empíricos.

Para avaliar o desempenho dos preditores e preditores empíricos consideramos três critérios. Primeiramente determinamos o melhor preditor (empírico), i.e., aquele que apresenta EQMS (EQME) mínimo. Como algumas vezes a diferença no EQMS (EQME) de dois ou mais preditores é pequena e nos estudos de simulação esperamos um certo grau de variabilidade, usamos como critério adicional o acréscimo relativo percentual $^{2}$ no EQMS (EQME) de cada preditor (empírico) com relação ao melhor preditor (empírico), para identificar situações em que dois preditores (empíricos) podem se considerar "equivalentes". Neste caso, consideramos como critério $A R P<$ $5 \%$ ou $A R P<15 \%$, conforme preditores ou preditores empíricos sejam considerados. Finalmente, identificamos os preditores (empíricos) que apresentam desempenho ruim $(A R P>50 \%)$ com relação ao melhor preditor (empírico).

Com a finalidade de apresentar mais claramente os resultados, vamos tratar separadamente as situações com variâncias intra-grupos iguais e diferentes.

\subsubsection{Variâncias intra-grupos iguais}

O estudo de simulação mostrou que os valores do EQMS e do EQME são apenas levemente afetados por mudanças na forma de distribuição da variável resposta. Em vista destes resultados restringimos a análise subseqüente aos casos 1. 4 e 5 (Tabela 2.1).

Um estudo preliminar mostron que os preditores empíricos obtidos sob o MSS com $\widehat{k}_{1}^{(S S)}$ e sob o MPA com $\widehat{k}_{1}^{(P A)}$ geralmente têm menor EQME que aqueles que

${ }^{2} \mathrm{O}$ acréscimo relativo percentual em $\mathrm{A}$ com relação a $\mathrm{B}$ é definido como $A R P=\frac{A-B}{B} \times 100 \%$ 
usam as constantes $\widehat{k}_{2}^{(S S)}$ e $\widehat{k}_{2}^{(P A)}$, respectivamente. Por isto, limitamos a análise aos primeiros.

\section{Componentes de variância conhecidas}

Embora os EQMS de cada preditor sejam obtidos assumindo uma estrutura de covariância diferente que depende do modelo, o MPA forneceu, em geral, os preditores com EQMS mínimo. Só em poucos casos os preditores obtidos sob o $M L M$ ou o $M S S$ apresentaram EQMS mínimo, geralmente para valores extremos $(0,01$ ou 0,99$)$ de $\rho_{s}$ e $\rho_{t}$. Nestes casos, o acréscimo relativo percentual no EQMS do preditor obtido sob o MPA com relação ao melhor preditor foi, no máximo, de $0,03 \%$, o que pode simplesmente ser explicado pela variabilidade introduzida pelo processo de simulação.

Para completar o estudo do desempenho dos diferentes preditores consideramos uma comparação relativa dos seus EQMS. Para as frações de amostragem aqui consideradas, a Tabela B.1 (Apêndice B) apresenta o máximo acréscimo relativo percentual no EQMS para a $M G$ ou os preditores obtidos sob o $M L M$, o $M S S$ ou o $M P A$ com relação ao melhor preditor (geralmente aquele obtido sob o $M P A$ ).

Fora o preditor obtido sob o $M P A$, o preditor obtido sob o $M L M$ está mais próximo do melhor preditor que a $M G$ ou o preditor obtido sob o $M S S$, apresentando menores valores do máximo acréscimo relativo percentual no EQMS (ver Tabela B.1, Apêndice B). À medida que o número de grupos e a fração de amostragem dos grupos aumentam, o acréscimo relativo percentual no EQMS também aumenta.

Também identificamos situações (dependendo dos coeficientes de correlação intraclasse) nas quais a $M G$ assim como os preditores obtidos sob o $M L M$. sob o MSS ou sob o MPA apresentam um comportamento similar (ARP <5\%) com relação ao preditor com EQMS mínimo. Na Tabela 2.2 apresentamos um resumo destes resultados para todas as frações de amostragem dos grupos e unidades. A tabela dá uma idéia geral das semelhanças e diferenças, as quais podem se estender dependendo do tamanho da população e dos grupos assim como das frações de amostragem.

A partir da Tabela 2.2 observamos que todos os preditores têm um desempenho semelhante quando ambos os coeficientes de correlação intra-classe são altos $\left(\rho_{s} \geq 0.95\right.$ e $\left.\rho_{t} \geq 0.5\right)$. O preditor com melhor desempenho ou equivalente a cle é 
Tabela 2.2: Preditores com desempenho semelhante a.o melhor preditor $(A R P<5 \%)$ para as populações com variâncias intra-grupos iguais

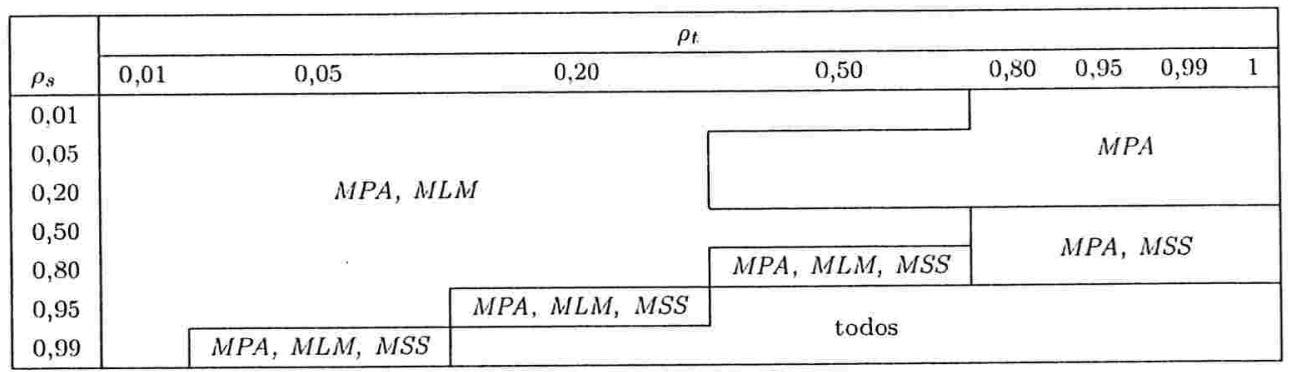

aquele obtido sob o $M P A$, independentemente dos coeficientes de correlação intraclasse e das frações de amostragem. O desempenho do preditor obtido sob o MLM é semelhante ao do melhor preditor sob uma ampla gama de condições (i.e. $\rho_{s} \geq 0,95$ ou $\left.\rho_{t} \leq 0,2\right)$, seguido pelo preditor obtido sob o MSS quando ambos os coeficientes de correlação intra-classe variam (de $\rho_{s} \geq 0,5$ e $\rho_{t} \geq 0,8$ a $\rho_{s} \geq 0,99$ e $\rho_{t} \geq 0,05$, com um coeficiente aumentando à medida que o outro diminui).

Além disso, identificamos situações nas quais a $M G$ assim como os preditores obtidos sob o $M L M$ ou o $M S S$ apresentam um comportamento ruim $(A R P>50 \%$ ). Nas Figuras B.1, B.2 e B.3 no Apêndice B apresentamos estes resultados para as populações com $N=10$ e $M=20$; para as outras populações obtemos um comportamento semelhante. O preditor obtido sob o $M L M$ apresenta um desempenho ruim quando o coeficiente de correlação intra-classe dos grupos é pequeno $\left(\rho_{s} \leq 0,2\right)$, o coeficiente de correlação intra-classe das unidades é alto $\left(\rho_{t} \geq 0,95\right)$ e a fração de amostragem das unidades é grande $(f \geq 0,8)$ (ver Figura B.2 no Apèndice B). A $M G$ e o preditor obtido sob o MSS apresentam comportamento ruim à medida que ambos os coeficientes de correlação intra-classe tendem conjuntamente a zero, mas isso acontece quando $f$ decresce no caso da $M G$ ou quando $f$ cresce no caso do preditor obtido sob o MSS (ver Figuras B.1. e B.3 no Apèndice B). Por outro lado, o preditor obtido sob o MPA nunca apresenta esse comportamento desfavorável. 


\section{Avaliação da perda devida ao uso dos preditores empíricos}

Para avaliar a perda associada ao uso dos preditores empíricos no lugar daqueles em que as variâncias são conhecidas, calculamos o acréscimo relativo percentual $(A R P)$ no EQME dos preditores empíricos com relação ao EQMS dos preditores obtidos sob cada modelo. Para o $M L M$ e o $M P A$, o EQME associado aos preditores empíricos sempre sobre-estima o EQMS, enquanto que para o preditor empírico obtido sob o MSS, o EMQE sobre-estima o EQMS em 85\% a $96 \%$ dos casos.

A Tabela 2.3 apresenta algumas estatísticas descritivas do ARP no EQME dos preditores empíricos com relação ao EQMS dos preditores obtidos sob cada modelo, para as populações simuladas e a Figura B.4 no Apêndice B mostra os "boxplots" correspondentes.

Tabela 2.3: Estatísticas descritivas do acréscimo relativo percentual $(A R P)$ no EQME dos preditores empíricos com relação ao EQMS dos preditores obtidos sob cada modelo, para as populações com variâncias intra-grupos iguais

\begin{tabular}{|c|c|r|r|r|r|r|r|c|}
\hline \hline População & Preditor & Mínimo & Q1 & Média & Mediana & Q3 & Máximo & $\begin{array}{c}\text { Desvio } \\
\text { padrão }\end{array}$ \\
\hline \hline \multirow{3}{*}{$N=10, M=5$} & $M L M$ & 0,1810 & 10,36 & 28,13 & 23,60 & 38,25 & 114,54 & 24,13 \\
& $M S S$ & $-5,1669$ & 1,50 & 10,98 & 5,89 & 14,65 & 64,34 & 14,19 \\
& $M P A$ & 0,0259 & 6,76 & 24,05 & 21,18 & 34,72 & 80,10 & 20,15 \\
\hline \hline \multirow{3}{*}{$N=10, M=20$} & $M L M$ & 0,0384 & 5,81 & 23,01 & 18,51 & 31,55 & 163,34 & 22,57 \\
& $M S S$ & $-5,5243$ & 0,70 & 12,83 & 4,51 & 16,36 & 119,45 & 20,07 \\
& $M P A$ & 0,0017 & 2,63 & 19,97 & 15,69 & 28,77 & 103,27 & 20,23 \\
\hline \hline \multirow{3}{*}{$N=50, M=20$} & $M L M$ & 0,0061 & 0,64 & 20,75 & 9,27 & 25,14 & 197,16 & 30,61 \\
& $M S S$ & $-10,9744$ & 0,02 & 9,01 & 0,43 & 5,66 & 154,27 & 23,99 \\
& $M P A$ & 0,0002 & 0,30 & 16,56 & 4,00 & 19,68 & 141,35 & 25,53 \\
\hline \hline
\end{tabular}

Em geral, o melhor desempenho corresponde ao preditor empírico obtido sob o $M S S$ que em $75 \%$ das situações apresenta $|A R P|<16 \%$, seguido pelo preditor empírico obticlo sob o MPA e, posteriormente, pelo preditor empírico obticlo sob - $M L M$ que apresentam ARP menores que $35 \%$ e $38 \%$ em $75 \%$ dos casos, respectivamente (ver Tabela 2.3). O pior desempenho é obtido quando as fraçooes de amostragens das midades são pequenas. 


\section{Componentes de variância desconhecidas}

Para simplificar a apresentação dos resultados sobre a avaliação do desempenho dos preditores empíricos, calculamos a porcentagem dos casos em que cada preditor satisfaz cada um dos três critérios estabelecidos previamente (EQME mínimo, "equivalência" com o melhor preditor e desempenho ruim). Todas estas porcentagens foram calculadas considerando as combinações de todas as correlações intraclasse e frações de amostragem das unidades no denominador, i.e., $168(=7 \times 8 \times 3)$ para as populações com $N=10$ e $M=5$ e $392(=7 \times 8 \times 7)$ para as outras (rotuladas $\forall \rho_{s}, \rho_{t}, f$ nas Tabelas B.2 e B.3 no Apêndice B). Estas porcentagens foram recalculadas excluindo os valores "extremos" dos coeficientes de correlação intraclasse $(0,01$ e 0,99$)$, pois eles podem tanto aumentar quanto diminuir a porcentagem de ocorrências em que os preditores apresentam comportamento similar (de acordo ao critério $0<A R P<15 \%)$ ou desempenho ruim $(A R P>50 \%)$. As colunas correspondentes nas Tabelas B.2 e B.3 no Apêndice B são rotuladas "Excluindo $\rho_{s}, \rho_{t}=0,01 ; 0,99 "$ ". Neste último caso, usamos 90 como denominador para as populações com $N=10$ e $M=5$; nas outras usamos 210. Em alguns casos a soma das porcentagens na Tabela B.2 excede 100\% (identificadas com *), pois os EQME para os preditores obtidos sob o MSS ou o MPA possuem exatamente o mesmo valor mínimo.

Quando consideramos o EQME mínimo, o preditor empírico obtido sob o MPA nem sempre apresenta o melhor desempenho. Na Tabela 2.4 mostramos os dois preditores empíricos que apresentam as maiores porcentagens de ocorrência de bom desempenho para cada tipo de população e fração de amostragem dos grupos. Maiores detalhes são apresentados na Tabela B.2 no Apêndice B.

Para as populações com $N=10$, a $M G$ apresenta EQME mínimo para pequenas frações de amostragem dos grupos $(F=0,2)$, seguida pelo preditor empírico obtido sob o MPA. Na medida que a fração de amostragem dos grupos aumenta, os preditores empíricos obtidos sob o MSS ou o MPA aparecem como os dois melhores. Para as populaçōes com $N=50$, a melhor altennativa é geralmente o preditor empírico obtido sob o MPA, seguido pelo preditor empírico obtido sob o MSS.

Independentemente das características das populações e das frações de amostragem, o preditor empírico obtido sob o MPA é o melhor ou equivalente ao me- 
Tabela 2.4: Porcentagem de casos em que os dois melhores preditores empíricos apresentam EQME mínimo, para as populações com variâncias intra-grupos iguais

\begin{tabular}{|c|l|c|c|c|c|c|}
\hline \multirow{3}{*}{ População } & \multicolumn{6}{|c|}{ Fração de amostragem dos grupos $F$} \\
\cline { 2 - 7 } & \multicolumn{2}{|c|}{0.2} & \multicolumn{2}{|c|}{0.5} & \multicolumn{2}{c|}{0.8} \\
\cline { 2 - 7 } & Preditor & $\%$ & Preditor & $\%$ & Preditor & $\%$ \\
\hline \multirow{2}{*}{$N=10, M=5$} & 1) $M G$ & $36^{n}-36^{b}$ & 1) MSS & $39-47$ & 1) MPA/MSS & $42-46$ \\
& 2) $M P A$ & $27-29$ & 2) MPA & $27-23$ & 2) MLM & $19-14$ \\
\hline \multirow{3}{*}{$N=10, M=20$} & 1) $M G$ & $47-50$ & 1) MSS & $49-59$ & 1) MSS & $52-60$ \\
& 2) MPA/MSS & $19-22$ & 2) MPA & $20-14$ & 2) MPA & $32-29$ \\
\hline \multirow{3}{*}{$N=50, M=20$} & 1) MSS & $46-53$ & 1) MPA & $50-51$ & 1) MPA & $56-60$ \\
& 2) MPA & $38-35$ & 2) MSS & $36-39$ & 2) MSS & $30-31$ \\
\hline \hline
\end{tabular}

${ }^{a}$ As primeiras porcentagens são calculadas para todas as correlações intra-classe e frações de amostragem das unidades.

${ }^{b}$ As segundas porcentagens são calculadas excluindo $\rho_{s}, \rho_{t}=0,01 ; 0,99$.

lhor para uma porcentagem de situações maior ( $90 \%$ a 100\%) que aquela associada a qualquer outro dos seus concorrentes (ver Tabela B.2 no Apêndice B).

A Tabela 2.5 mostra as situações (dependendo de $\rho_{s}$ e $\rho_{t}$ ) em que os preditores podem se considerar equivalentes $(A R P<15 \%)$ ao melhor preditor empírico, para todas as frações de amostragem de grupos e de unidades. As regiōes que nela aparecem podem se estender dependendo do número e tamanho dos grupos assim como das frações de amostragem de grupos e de unidades.

Tabela 2.5: Preditores empíricos com desempenho semelhante ao melhor preditor empírico $(A R P<15 \%)$, para as populações com variâncias intra-grupos iguais

\begin{tabular}{|c|c|c|c|c|c|c|c|c|}
\hline \multirow[b]{2}{*}{$\rho_{s}$} & \multicolumn{8}{|c|}{$\rho_{t}$} \\
\hline & 0,01 & 0,05 & 0.20 & 0,50 & 0,30 & 0,95 & 0.99 & 1 \\
\hline \multicolumn{9}{|l|}{0,01} \\
\hline 0,05 & \multirow[t]{3}{*}{$M P A$} & & & \multirow{2}{*}{$M P A$} & & & & \\
\hline 0.20 & & \multirow{2}{*}{\multicolumn{2}{|c|}{$M P A, M L M$}} & & \multirow{2}{*}{\multicolumn{4}{|c|}{ MPA. .MSS }} \\
\hline 0,50 & & & & & & & & \\
\hline 0,80 & & & MPA. ML.M. MSS & & \multirow{2}{*}{\multicolumn{4}{|c|}{ MPA. MSS MG }} \\
\hline 0,95 & & MPA. ML.M, MSS & & & & & & \\
\hline 0,91 & MPA. MLM MSS & \multicolumn{7}{|c|}{ todos } \\
\hline
\end{tabular}

A partir da Tabela 2.5 podemos observar que a região em que a $M G$ e o preditor empírico obtido sob o MSS são equivalentes ao melhor preditor empírico expandiu- 
se com relação ao caso de variâncias conhecidas; no entanto ela se reduziu para o preditor empírico obtido sob o $M L M$.

Também é importante destacar que, analogamente ao caso de variâncias conhecidas, tanto a $M G$ quanto o preditor empírico obtido sob o $M S S$ apresentam comportamento ruim $(A R P>50 \%)$ em um número considerável de situações (5\%$47 \%$ e 10\%-32\%, respectivamente), seguidos pelo preditor empírico obtido sob o $\operatorname{MLM}$ (4\%-7\%) (ver Tabela B.3 no Apêndice B). Em particular, este último preditor apresenta comportamento ruim quando o coeficiente de correlação intra-classe dos grupos é pequeno $\left(\rho_{s} \leq 0,5\right)$, o coeficiente de correlação intra-classe das unidades é grande $\left(\rho_{t} \geq 0,8\right)$ e a fração de amostragem das unidades é grande $(f \geq 0,6)$. Para este preditor, o máximo $A R P$ é de $780 \%$. O preditor empírico obtido sob o $M S S$ e a $M G$ apresentam desempenho ruim quando ambos os coeficientes de correlação se aproximam de zero, mas isto acontece para $f$ grande no caso dos preditores obtidos sob o $M S S$ e para $f$ pequeno no caso da $M G$. O máximo $A R P$ é de $1676 \%$ para o preditor empírico obtido sob o $M S S$ e de $2038 \%$, para a $M G$. Pelo contrário, o preditor empírico obtido sob o MPA nunca apresenta comportamento ruim o que sugere que, na prática, ele não oferece tanto risco quanto os seus competidores.

\subsubsection{Variâncias intra-grupo diferentes}

Um estudo preliminar mostrou que os preditores empíricos obtidos sob o MSS com $\widehat{k}_{i 3}^{(S S)}$ geralmente têm maior EQME que aqueles que usam as constantes $\widehat{k}_{i 1}^{(S S)} \mathrm{e}$ $\widehat{k}_{i 2}^{(S S)}$. Devido a isto, restringimos a análise a estes dois últimos.

\section{Componentes de variância conhecidas}

Do mesmo modo que no caso de variâncias intra-grupo iguais e conlecidas, o MPA forneceu, em geral, os preditores com EQMS mínimo. Também aqui os preditores obtidos sob o MLM ou o MSS apresentaram EQMS mínimo em algumas ocasiōes. geralmente para valores de $\rho_{s}$ e $\rho_{t}$ extremos (0.01 ou 0,99), mas o ARP no EQMS do preditor obtido sob o MPA com relaçāo ao melhor preditor é. no máximo. de $0.2 \%$.

Do mesmo modo que para o caso de variancias intra-grupo iguais e conhecidas. consideramos uma comparação relativa dos EQMS dos diferentes preditores. Para as 
frações de amostragem aqui consideradas, a Tabela B.4 no Apêndice B apresenta o máximo acréscimo relativo percentual no EQMS para a $M G$ ou os preditores obtidos sob o $M L M$, o $M S S$ ou o MPA com relação ao melhor preditor (geralmente aquele obtido sob o MPA). Os resultados obtidos são muito parecidos com aqueles em que as variâncias intra-grupos são iguais e o preditor obtido sob o $M P A$ sempre apresenta o menor $A R P$. Também identificamos situações em que a $M G$ e os preditores obtidos sob o $M L M$, sob o $M S S$ ou sob o $M P A$ têm desempenho similar ao melhor preditor (geralmente aquele obtido sob o MPA). Resumimos os resultados na Tabela 2.6, a partir da qual observamos um padrão quase igual ao caso de variâncias intra-grupos iguais e conhecidas.

Tabela 2.6: Preditores com desempenho semelhante ao melhor preditor $(A R P<5 \%)$ para as populações com variâncias intra-grupos diferentes

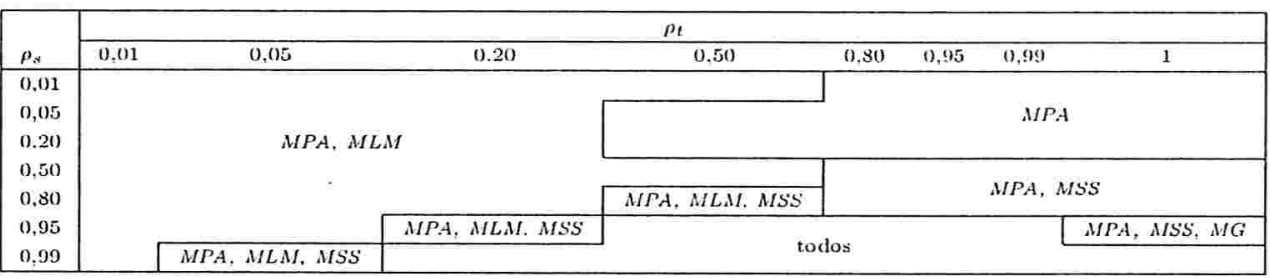

Além disso, determinamos em que situações a $M G$ e os preditores obtidos sob o $M L M$ e sob o $M S S$ exibem um comportamento inferior $(A R P>50 \%)$ com relação ao melhor preditor. Estas situações foram análogas àquelas correspondentes ao caso de variâncias intra-grupo iguais.

\section{Avaliação da perda devida ao uso dos preditores empíricos}

Como no caso de variâncias intra-grupos iguais, calculamos o acréscimo relativo percentual $(A R P)$ no EQME dos preditores empíricos com relação ao EQMS dos preditores obtidos sob cada modelo. Para o MLM e o MPA, o EQME associado aos preditores empíricos sempre sobre-estima o EQMIS. O EQME dos preditores

empíricos obtidos sob o MSS com as constantes $\widehat{k}_{i 1}^{(S S)}$ ou $\widehat{k}_{i 2}^{(S S)}$ sobre-estima o EQMS em $85 \%$ a $100 \%$ ou $75 \%$ a $100 \%$ dos casos, respectivamente.

A Tabela 2.7 apresenta algumas estatísticas descritivas do ARP no EQME dos 
preditores empíricos com relação ao EQMS dos preditores obtidos sob cada modelo, para as populações estudadas. Os preditores empíricos obtidos sob o $M L M \mathrm{com}$ a constante $\widehat{k}_{i 2}^{(L M)}$ ou sob o MSS com a constante $\widehat{k}_{i 2}^{(S S)}$ apresentam ARP médios superiores ao 100\% indicando que, em média, o EQME supera o EQMS pelo menos em dobro. Em geral, o melhor desempenho corresponde ao preditor empírico obtido sob o $M S S$ com a constante $\widehat{k}_{i 1}^{(S S)}$, que em $75 \%$ das situações apresenta um $|A R P|<$ $25 \%$, seguido pelo preditor empírico obtido sob o $M P A$, que apresenta um $A R P$ menor a $35 \%$ em $75 \%$ dos casos.

Tabela 2.7: Estatísticas descritivas do acréscimo relativo percentual $(A R P)$ no EQME dos preditores empíricos com relação ao EQMS dos preditores obtidos sob cada modelo, para as populações com variâncias intra-grupos diferentes

\begin{tabular}{|c|c|c|c|c|c|c|c|c|}
\hline População & Preditor & Mínimo & Q1 & Média & Mediana & Q3 & Máximo & $\begin{array}{l}\text { Desvio } \\
\text { padräo }\end{array}$ \\
\hline \multirow{5}{*}{$N=10, M=5$} & $M L M 1^{\prime \prime}$ & 0,565 & 20,80 & 60,45 & 43,21 & 71,88 & 328,55 & 62,77 \\
\hline & $M L M 2^{b}$ & 2,034 & 24,72 & 1133,93 & 41,20 & 123,61 & 43265,32 & 4598,86 \\
\hline & $M S S 1^{\circ}$ & $-3,213$ & 2,80 & 18,38 & 9.59 & 24,95 & 113,10 & 24,04 \\
\hline & MSS $2^{d i}$ & $-6,952$ & 3,38 & 128,79 & 10,40 & 31,89 & 2609,74 & 421,00 \\
\hline & MPA & 0,029 & 6,83 & 24,06 & 21,19 & 34,73 & 80,10 & 20,14 \\
\hline \multirow{5}{*}{$N=10, M=20$} & $M L M 1$ & 0,059 & 7,19 & 36,21 & 23,62 & 41,46 & 329,61 & 48,65 \\
\hline & MLM2 & 0,852 & 23,22 & 4689,89 & 43,04 & 297,54 & 391435,43 & 26370,49 \\
\hline & MSS1 & $-3,794$ & 0,95 & 21,63 & 5,90 & 21,13 & 292,62 & 43,54 \\
\hline & MSS2 & $-6,779$ & 3,09 & 421,86 & 17,26 & 57,71 & 10816,45 & 1475,85 \\
\hline & $M P A$ & 0,002 & 2,70 & 19.98 & 15,66 & 28,77 & 103,27 & 20,22 \\
\hline \multirow{5}{*}{$N=50, M=20$} & $M L M 1$ & 0,023 & 1,97 & 61,22 & 16,90 & 44,22 & 1488,60 & 156,76 \\
\hline & MLM2 & 8,715 & 43,82 & 9546,05 & 118,91 & 1546,37 & 523174,07 & $435.44,88$ \\
\hline & $M S S 1$ & $-9,576$ & 0,07 & 35,54 & 1,45 & 13,17 & 990,17 & 116,21 \\
\hline & MSS2 & $-11,169$ & 1,65 & 849,96 & 29,23 & 266.29 & 14621,09 & 2417,11 \\
\hline & $M P A$ & $-0,001$ & 0.32 & 16,57 & 4,01 & 19,68 & 141,35 & 25.83 \\
\hline
\end{tabular}

"MLM1 denota o preditor empírico obtidlo sob o $M L M$ com a constante $\widehat{k}_{i 1}^{(L, M)}$

${ }^{b} M L M 2$ denota o preditor empírico obtido sob o $M L M$ com a constante $\hat{k}_{i 2}^{(L M)}$

cMSS1 denota o preditor empírico obtido sob o MSS com a constante $\widehat{k}_{i 1}^{(S S)}$

"MSS2 denota o preditor empírico obtido sob o MSS com a constante $\widehat{k}_{i 2}^{(S S)}$

\section{Componentes de variância desconhecidas}

Quando consideramos o EQIE mínimo, o preditor empírico obtido sob o MPA nem sempre apresenta o melhor desempenho. Na Tabela 2.8 mostramos os dois 
preditores empíricos que apresentam as maiores porcentagens de ocorrência de bom desempenho para cada tipo de população e fração de amostragem dos grupos.

Tabela 2.8: Porcentagem de casos em que os dois melhores preditores empíricos apresentam EQME mínimo, para as populações com variâncias intra-grupos diferentes

\begin{tabular}{|c|c|c|c|c|c|c|}
\hline \multirow[b]{3}{*}{ População } & \multicolumn{6}{|c|}{ Fração de amostragem dos grupos $F$} \\
\hline & \multicolumn{2}{|l|}{0.2} & \multicolumn{2}{|l|}{0.5} & \multicolumn{2}{|l|}{0.8} \\
\hline & Preditor & $\%$ & Preditor & $\%$ & Preditor & $\%$ \\
\hline \multirow{2}{*}{$N=10, M=5$} & 1) $M G$ & $36^{a}-19^{b}$ & 1) $M L M 2$ & $38-15$ & 1) $M P A$ & $49-28$ \\
\hline & 2) $M P A$ & $29-14$ & 2) $M G / M S S 2$ & $26-14$ & 2) $M L M 2$ & $35-13$ \\
\hline \multirow{2}{*}{$N=10, M=20$} & 1) $M G$ & $47-50$ & 1) $M L M 2 / M S S 2$ & $27-25$ & 1) $M / P A$ & $51-55$ \\
\hline & 2) $M P A / M S S 2$ & $19-22$ & 2) $M G / M S S 1$ & $20-22$ & 2) $M L M 2 / M S S 2$ & $27-21$ \\
\hline \multirow{2}{*}{$N=50, M=20$} & 1) $M P A$ & $61-65$ & 1) $M P A$ & $84-90$ & 1) $M P A$ & $89-94$ \\
\hline & 2) $M L M 2 / M S S 2$ & $25-19$ & 2) $M L M 2 / M S S 2$ & $11-6$ & 2) $M L M 2 / M S S 2$ & $6-6$ \\
\hline
\end{tabular}

"As primeiras porcentagens são calculadas para todas as correlações intra-classe e frações de amostragem das unidades.

${ }^{b}$ As segundas porcentagens são calculadas excluindo $\rho_{s}, \rho_{t}=0,01 ; 0,99$.

Para as populações com $N=10$, a $M G$ apresenta EQME mínimo para frações de amostragem dos grupos pequenas $(F=0,2)$. Quando esta fração aumenta para $F=0,5$, os preditores empíricos obtidos sob o $M L M$ com a constante $\widehat{k}_{i 2}^{(L M)}$ ou sob o $M S S$ com a constante $\widehat{k}_{i 2}^{(S S)}$ são os que apresentam as maiores porcentagens de situações com EQME mínimo, embora estas porcentagens não superem $40 \%$. Entretanto, para grandes frações de amostragens de grupos $(F=0.8)$, o preditor empírico com melhor desempenho é aquele obtido sob o MPA. Para as populações com $N=50$, o melhor desempenho corresponde sempre ao preditor empírico obtido sob o MPA, que apresenta EQME mínimo entre $60 \%$ e $95 \%$ dos casos, com porcentagens maiores à medida que a fraçào de amostragem dos grupos aumenta.

Independentemente das características das populações e das frações de amostragem, o preditor empírico obtido sob o MPA é o melhor on equivalente ao melhor para uma porcentagem grande de situações ( $76 \%$ a $100 \%)$, superando sempre a qualquer 1 un dos seus concorrentes (ver Tabela B.5 no Apèndice B).

Também é importante destacar que tanto a $M G$ quanto os preditores empíricos obtidos sob o MSS com a constante $\widehat{k}_{i 2}^{(S S)}$ on sob o MLM com a constante $\widehat{h}_{i 2}^{(L, M)}$ apresentam comportamento rum $(A R P>50 \%) \mathrm{em}$ um número considerável de 
situações (até 48\%, 57\% e 68\%, respectivamente) (ver Tabela B.6 no Apêndice B). Em particular, estes dois últimos preditores têm um comportamento ruim quando ambos os coeficientes de correlação intra-classe $\left(\rho_{s}\right.$ e $\left.\rho_{t}\right)$ aumentam, apresentando $A R P$ máximos excesivamente grandes (ver Tabela B.6). Isto mostra que, ainda que sejam os melhores preditores em algumas situações, eles são muito instáveis, apresentando desempenho muito ruim em outras.

Os preditores empíricos obtidos sob o MSS com a constante $\widehat{k}_{i 1}^{(S S)}$ ou sob $M L M$ com a constante $\widehat{k}_{i 1}^{(L M)}$ também apresentam comportamento ruim $(A R P>50 \%)$ em algumas situações (até 35\% e 43\%, respectivamente) com ARP máximos de $1680 \%$ e $720 \%$, respectivamente (ver Tabela B.6). Por outro lado, o preditor empírico obtido sob o MPA nunca apresenta comportamento ruim.

\subsection{Discussão e conclusões}

Quando as variâncias são conhecidas, o preditor obtido sob o MPA geralmente apresenta EQMS mínimo, ainda no caso em que as variâncias dentro dos grupos são diferentes.

A forma da distribuição da variável resposta quase não tem efeito sobre os valores do EQMS ou do EQME.

Na ausência de erro na resposta e quando são amostradas todas as unidades de cada grupo selecionado, os preditores empíricos obtidos sob o MSS ou sob o MPA reproduzem a média do grupo, entanto que o preditor empírico obtido sob o $M L M$, não. Isto destaca sua dependência da suposição de população infinita.

Tanto no caso de variâncias intra-grupo iguais quanto diferentes, o preditor empírico obtido sob o MPA melhora o seu desempenho à medida que o número, o tamanho e a fração de amostragem dos grupos aumenta. Ele apresenta um comportamento mais estável que os seus concorrentes, sendo o melhor ou equivalente ao melhor preditor empírico em $76 \%-100 \%$ das situações. Ainda mais, ele é o único preditor empírico que não apresenta desempenho ruim (i.e.. ARP no EQ.IE com relação ao EQME do melhor preditor empírico superior a 50\%).

No caso de variancias intra-grupos diferentes e desconhecidas, a instabilidade dos preditores empíricos obtidos sob o MSS ou sob o MLM poderia se explicar pelo 
fato de que estes preditores requerem que una constante de encolhimento diferente seja estimada para cada grupo amostrado e a estimação de tal constante é feita com base na informação do grupo, diferentemente do preditor empírico obtido sob o $M P A$, em que apenas uma constante precisa ser estimada com base na informação de todos os grupos amostrados.

Finalmente, tendo em mente as limitações dos estudos de simulação e percebendo que podem ser necessárias pesquisas adicionais para obter conclusões mais definitivas, os nossos resultados apontam na direção de recomendar o preditor empírico obtido sob o MPA em oposição aos seus competidores para uma ampla gama de condições. 


\section{Capítulo 3}

\section{Preditores ótimos sob amostragem com dois estágios de populações finitas: variáveis discretas}

\subsection{Introdução}

No capítulo anterior vimos que os preditores empíricos obtidos sob o modelo de permutação aleatória $(M P A)$ têm um desempenho mais estável que os obtidos sob o $M L M$ ou sob o modelo de Scott e Smith $(M S S)$, ainda no caso de variâncias intra-grupos diferentes. Além disso, seu desempenho melhora quando o número, tamanho e fração de amostragem dos grupos aumenta. Duas vantagens desse modelo, além da consideração da estrutura finita da população, são sua natureza intuitiva e sua simplicidade. Como só se baseia no planejamento amostral, não necessita suposições distribucionais além da existência dos dois primeiros momentos e pode ser aplicado tanto no caso de variáveis contínuas quanto discretas. Quando a variável resposta é discreta, o MLM não é apropriado, mas podemos usar o modelo linear misto generalizado ( $M L M G$ ) (ver Mc Culloch e Searle (2001) on Fahrmeir e Tutz (1994), entre outros). Do mesmo modo que no $M L M$, sob este modelo, assume-se que a população é infinita. Além disso, as inferências são baseadas em resultados assintóticos, de modo que sua aplicação prática requer tamanhos amostrais grandes. Também. os preditores obtidos sob este modelo geralmente não têm uma forma fe- 
chada e precisam ser calculados mediante procedimentos iterativos, o que algumas vezes ocasiona problemas de convêrgencia.

Como variáveis discretas são muito freqüêntes na prática, nosso objetivo neste capítulo é comparar o desempenho dos preditores empíricos obtidos sob o $M L M G$, sob o $M S S$ e sob o MPA para este tipo de variáveis, por meio de um estudo de simulação.

\subsection{O modelo linear misto generalizado ( $M L M G$ ) para populações infinitas}

Sob o $M L M G$, considera-se que os dados obtidos mediante uma amostragem em dois estágios vêm de uma população infinita. Denotemos por $Y_{S}=\left(Y_{S 1}^{\prime}, \ldots, Y_{S_{n}}^{\prime}\right)^{\prime}$ o vetor de respostas em que, para as unidades no $i$-ésimo grupo amostrado, $Y_{S i}=\left(Y_{i 1}, \ldots, Y_{i m}\right)^{\prime}, i=1, \ldots, n$. Num primeiro estágio, sob o $M L M G$ assume-se que, dados os efeitos aleatórios $B=\left(B_{1}, \ldots, B_{n}\right)^{\prime}$, as respostas $\left(Y_{i j}\right)$ podem ser modeladas por

$$
Y_{i j} \mid B \stackrel{\text { ind. }}{\sim} f\left(Y_{i j} \mid B\right)
$$

em que $f\left(Y_{i j} \mid B\right)$ é uma densidade da família exponencial, i.e.,

$$
f\left(Y_{i j} \mid B\right)=\exp \left\{\phi\left[Y_{i j} \theta_{i j}-b\left(\theta_{i j}\right)\right]-c\left(Y_{i j}, \phi\right)\right\}, \quad i=1, \ldots, n, j=1, \ldots, m
$$

com

$\theta_{i j} \in \Theta \subset \mathbb{R}$ denotando o parâmetro natural da família,

$\phi>0$ indicando o parâmetro de dispersão e

$b($.$) e c() \geq$.0 representando funçoes convenientes.

Note que se assume independência condicional das observações dentro e entre grupos. A média e a variancia condicional de $Y_{i j}$ estão relacionadas com $\theta_{i j}$ em (3.1) por intermédio das identidades $E\left(Y_{i j} \mid B_{i}\right)=\mu_{i j}=\partial b\left(\theta_{i j}\right) / \partial \theta_{i j}$ e $\operatorname{Var}\left(Y_{i,} \mid B_{i}\right)=\sigma_{i, j}^{2}=$ $\phi \partial^{2} b\left(\theta_{i j}\right) / \partial \theta_{i j}^{2}$. Para uma transformação monótona da média, $g($.$) , assume-se um$ modelo linear representado por

$$
g(\boldsymbol{\mu})=\left(g\left(\mu_{11}\right), \ldots g\left(\mu_{n m}\right)\right)^{\prime}=\mathbf{X}_{S} \mu^{*}+\mathbf{Z}_{S} B
$$


em que $\mathrm{X}_{S}=1_{n m}$ e $\mathrm{Z}_{S}=\mathrm{I}_{n} \otimes 1_{m}$ são as matrizes de incidência associadas aos efeitos fixos e aleatórios, respectivamente. Num segundo estágio, assume-se uma distribuição específica (geralmente $B \sim N(\mathbf{0}, \mathbf{G})$ ) para os efeitos aleatórios $B_{i}$.

Para contagens, é particularmente comum assumir que $Y_{i j} \mid B_{i} \stackrel{\text { ind. }}{\sim} \operatorname{Poisson}\left(\mu_{i j}\right)$, $g(\mu)=\log (\mu)$ e $\mathbf{G}=\sigma^{2} \mathbf{I}_{n}$. Neste caso, devido à estrutura simples de $\mathrm{Z}_{S}$ e assumindo normalidade dos efeitos aleatórios, pode-se mostrar que, marginalmente, $\operatorname{Var}\left(Y_{i j}\right)>E\left(Y_{i j}\right)$, de modo que a distribuição marginal não é Poisson (McCulloch and Searle (2001)). Além disso, as respostas dentro do mesmo grupo são correlacionadas enquanto que as de diferentes grupos, não.

\subsection{Preditores}

Os preditores e preditores empíricos do valor latente $\left(T_{i}\right)$ do $i$-ésimo grupo amostrado, $i \leq n$, obtidos sob os modelos para populações finitas foram especificados nas seções (2.5) e (2.6) do capítulo 2.

Para o $M L M G, T_{i}$ corresponde a $g^{-1}\left(\mu^{*}+B_{i}\right)$. Geralmente não existe forma fechada para obter os preditores correspondentes e deve se usar um processo iterativo que, algumas vezes, causa problemas de convergência. Além disso, existem várias formas de obter os preditores e preditores empíricos, tais como aquelas baseadas em quasi-verossimilhança penalizada (Schall (1991), Breslow e Clayton (1993), Wolfinger e O'Connell (1993)), em quasi-verossimilhança penalizada corrigida (Breslow e Lin(1995), Lin e Breslow (1996)), em alguns enfoques Bayesianos cuja implementação exige algoritmos do tipo EM (Fahrmeir e Tutz (1994), Stiratelli, Laird e Ware, 1984), ou amostrador de Gibbs (Zeger e Karim (1991)) ou numa técnica que combina estimadores empíricos bayesianos com funções de estimação (Waclawiw e Liang (1993)). Embora nem sempre funcione bem na prática, especialmente para dados binários em grupos pequenos, vamos usar o método de quasi-verossimilhança penalizada, devido a sua disponibilidade computacional, tanto em SAS quanto em $\mathrm{R} /$ Splus. 


\subsection{Estudo por simulação}

Considerando uma amostragem em dois estágios balanceada numa população finita, realizamos um estudo de simulação para comparar os erros quadráticos médios (EQM) dos preditores e preditores empíricos obtidos sob os diferentes modelos. A simulação consistiu em três passos: 1) a geração da população finita, 2) a seleção das amostras em dois estágios, e 3) a obtenção dos preditores empíricos e seus EQM. Consideramos somente situações sem erro na resposta, pois sua incorporação no $M L M G$ ainda requer de pesquisas adicionais (incluindo a incorporação de erros na resposta discretos).

\subsubsection{Geração da população finita}

Consideramos populações compostas por $N$ grupos com $M$ unidades cada um diferindo: 1) no número, $N$, e no tamanho, $M$, dos grupos, 2) na suposição de variâncias intra-grupos iguais, 3$)$ no coeficiente de correlação intra-classe $\left(\rho=\frac{\sigma^{2}}{\sigma^{2}+\sigma_{e}^{2}}\right)$, e 4) na forma da distribuição da média dos grupos.

Para gerar as populações com variâncias intra-grupos diferentes, procedemos da seguinte forma:

1. Consideramos 5 valores de coeficientes de correlação intra-classe $(\rho=0,1 ; 0,25$; $0,5 ; 0,75$ e 0,9$)$, fixando os valores iniciais de $\sigma^{2}$ e $\sigma_{e}^{2}$.

2. Para cada valor de $\rho$, geramos $N$ médias de grupos, $\lambda_{s}(s=1, \ldots, N)$, baseando-nos nos percentis de alguma distribuição conhecida. Dividimos o intervalo $[0,1]$ em $N+1$ intervalos do mesmo comprimento e obtivemos os percentis correspondentes ao limite superior de cada intervalo de acordo com a distribuição de probabilidade hipotética. Nesse contexto, consideramos duas distribuções: a lognormal e a uniforme.

3. Geramos as respostas das unidades com base nos percentis da distribuição Poisson com média $\lambda_{s}, s=1, \ldots, N$.

4. A partir clas respostas associadas às unidades, calculamos os valores reais das médias dos grupos, $\mu_{s}$, das variancias dentro dos grupos. $\sigma_{s}^{2}$, da variància entre grupos, $\sigma^{2}$ e de $\rho$. 
5. Como os valores reais em geral não concordaram com os valores inicias, repetimos todo o processo ajustando os valores iniciais até obter os valores específicos de $\rho$.

O procedimento para gerar as populações com variâncias intra-grupo iguais difere do anterior nos seguintes pontos:

2. Para cada valor de $\rho$, geramos as $M$ respostas das unidades para um grupo com base nos percentis da distribuição Poisson.

3. Obtivemos as respostas das unidades correspondentes aos outros grupos adicionando uma constante inteira. Este processo garantiu variâncias intra-grupo iguais. Permitimos também que alguns grupos tivessem a mesma média.

Geramos 45 populações: $15 \operatorname{com} N=10$ e $M=5,15 \operatorname{com} N=10$ e $M=20$ e $15 \mathrm{com} N=50$ e $M=20$. Em cada caso, as 15 populações corresponderam às combinações dos 5 valores do coeficiente de correlação intra-classe com os três tipos de estruturas populacionais: i) com variâncias intra-grupo diferentes e médias obtidas de uma distribuição lognormal (que denominaremos populações do tipo 1), ii) com variâncias intra-grupo diferentes e médias obtidas de uma distribuição uniforme (que denominaremos populações do tipo 2) e iii) com variâncias intra-grupo iguais.

\subsubsection{Amostragem em dois estágios}

Para cada população gerada, obtivemos uma amostra aleatória simples sem reposição de $n$ rótulos a partir de uma lista com os rótulos dos grupos $(1, \ldots, N)$. Combinamos a amostra de rótulos dos grupos com os dados populacionais e escollimos, sem reposição, uma amostra aleatória simples de $m$ unidades de cada um dos grupos previamente selecionados. Vamos nos referir a todo este processo como $11 m$ ensaio.

Para conduzir as amostragens em dois estágios sem reposição em cada população com $N=10$ e $M=5$ consideramos 3 frações de amostragem de grupos ( $F=\frac{n}{N}=$ $0,2: 0.5$ e 0,8$)$ e 3 fraçōes de amostragem de unidades $\left(f=\frac{m}{M}=0,4 ; 0,6\right.$ e 0.8 ). Portanto, usamos 9 planos amostrais para cada população gerada. Para as outras 
populações, usamos as mesmas 3 frações de amostragem de grupos, combinadas com 7 frações de amostragem de unidades $(f=0,1 ; 0,2 ; 0,4 ; 0,5 ; 0,6 ; 0,8$ e 0,9$)$, resultando em 21 planos amostrais para cada população gerada. No total, simulamos 765 populações/planos amostrais. Para cada população e cada plano obtivemos 1000 réplicas.

\subsubsection{Preditores}

Uma vez obtidas as amostras em dois estágios, calculamos os preditores e preditores empíricos sob cada modelo descrito nas seções 2.5 e 2.6 (sob o MPA ou sob o $M S S$ ) ou na seção 3.3 (sob o $M L M G$ ) e seus respectivos EQM. Para o $M L M G$ e o $M S S$, assumimos que os valores conhecidos de $\sigma^{2}$ e $\sigma_{e}^{2}$ correspondem à variância entre grupos e a média das variâncias intra-grupos, respectivamente. Na comparação, também incluimos a média do grupo $(M G)$.

Para o caso de variâncias conhecidas denotamos por EQMS o erro quadrático médio preditivo obtido por simulação sob as suposições de cada modelo particular e para o caso de variâncias desconhecidas, por EQME, aquele obtido por simulação.

Na ausência de erro na resposta, $\widehat{k}_{1}^{(P A)}=\widehat{k}_{2}^{(P A)}=\widehat{k}^{(P A)}$. Além disso, no caso de variâncias intra-grupos iguais, $\widehat{k}_{2}^{(S S)}$ só difere de $\widehat{k}^{(P A)}$ quando $Q M E=0$. Para o $M L M G$, os preditores empíricos foram obtidos usando a sub-rotina GLIMMIX para. a versão 8.0 do sistema SAS (Littel et al. (1996)). Para escolher as amostras em dois estágios, obter os preditores empíricos e seus respectivos EQM foi desenvolvida uma sub-rotina no sistema SAS (idmodeldisc) que está disponível na página www.umass.edu/cluster/ed.

\subsection{Resultados}

Para apresentar os resultados com maior clareza, vamos tratar separadamente as situações em que as componentes de variância são conhecidas ou desconhecidas.

Analogamente ao caso de variáveis contínuas. vamos avaliar o desempenho de cada preditor (empírico) por meio de três critérios: 1) a porcentagem de ocorrèncias em que o EQMS (EQME) é mínimo, 2) a porcentagem de situações em que cada preditor (empírico) é "equivalente" $(A R P<5 \%$ ou $A R P<10 \%$ segundo se trate 
dos preditores ou dos preditores empíricos) ao melhor preditor (empírico) e 3) a porcentagem de situações em que cada preditor (empírico) apresenta desempenho ruim $(A R P>50 \%)$ com relação ao melhor preditor (empírico).

Quando calculamos o EQME para o preditor empírico obtido sob o MLMG, encontramos problemas de convergência para pequenas frações de amostragem das unidades $(f=0,4$ para populações com $M=5$ e $f=0,1$ para populações com $M=20$ ). Em aproximadamente $50 \%$ dos casos, a convergência foi muito lenta, requerendo um número muito grande de passos. Nos outros $50 \%$ dos casos, o processo não convergiu. Isto aconteceu independentemente da fração de amostragem dos grupos considerada. Como consequiência, decidimos não incluir estas situações na comparação, apesar de isto representar uma desvantagem importante do preditor empírico obtido sob o $M L M G$ com relação aos outros preditores.

\subsubsection{Componentes de variância conhecidas}

\section{Variâncias intra-grupo diferentes}

Para as populações do tipo 1, o preditor obtido sob o MPA é o melhor em 57\%$100 \%$ dos casos, seguido somente pelo preditor obtido sob o $M L M G$ que apresenta. EQMS mínimo em até $43 \%$ dos casos. O melhor desempenho do preditor obtido sob o $M P A$ acontece para as populações com $N=10$ e aumenta à medida que a fração de amostragem dos grupos cresce.

Para as populações do tipo 2, o $M S S$ fornece o melhor preditor em $50 \%-80 \%$ dos casos, seguido exclusivamente pelo preditor obtido sob o MPA em $20 \%-50 \%$ dos casos.

O preditor obtido sob o MPA destaca-se como o único com desempenho melhor ou equivalente ao melhor para todas as situações. independentemente do tipo de população ou das fraçōes de amostragem dos grupos.

Somente a $M G$ ou o preditor obtido sob o $M L M G$ apresentam desempenho ruim. Para a $M G$, este desempenho inferior geralmente acontece quando $\rho \leq 0.25$ e $f \leq 0,4$. atingindo um $A R P$ máximo de $166 \%$. Para o preditor obtido sob o $M L M G$, este desempenho ruim verifica-se principalmente para as populaçóes de tipo 2, quando $\rho \leq 0,25$ e $f \geq 0,5$, apresentando $u m$ ARP máximo de $462 \%$. 


\section{Variâncias intra-grupo iguais}

O preditor obtido sob o MPA é o melhor em $40 \%-70 \%$ dos casos, principalmente para as populações $\operatorname{com} M=5$. O outro preditor que apresenta um bom desempenho em 30\%-50\% dos casos é aquele obtido sob o $M L M G$.

Novamente aqui, o preditor com melhor desempenho ou equivalente a ele é aquele obtido sob MPA em $97 \%-100 \%$ das situações, independentemente das frações de amostragem dos grupos.

Outra vez os preditores que apresentam desempenho ruim são a $M G$ e aquele obtido sob o $M L M G$. A primeira apresenta desempenho ruim quando $\rho \leq 0,25 \mathrm{e}$ $f \leq 0,4$, com um $A R P$ máximo de $172 \%$, enquanto o segundo mostra este desempenho desfavorável quando $\rho=0,25$ e $f=0,9$, atingindo um $A R P$ máximo de $83 \%$.

\subsubsection{Componentes de variância desconhecidas}

\section{Variâncias intra-grupo diferentes}

O melhor desempenho de cada um dos preditores empíricos depende das características da população e das frações de amostragem. Na Tabela 3.1 apresentamos os preditores empíricos que atingem as maiores porcentagens de ocorrências de EQME mínimo.

A partir da Tabela 3.1 observamos que para as populações com $N=10$, quando o tamanho do grupo ou a fração de amostragem de grupos é pequena ( $M=5$ ou $F=0,2$ ), o melhor preditor empírico é a $M G$ ou aquele obtido sob o $M S S$ com a constante $\widehat{k}_{i 2}$ em $40 \%-73 \%$ ou $40 \%$ dos casos, respectivamente. Quando $N, M$ e $F$ aumentam, os dois melhores preditores empíricos são os obtidos sob o MPA on sob o MSS com a constante $\widehat{k}_{i 1}^{(S S)}$ em $43 \%-90 \%$ ou $43 \%$ dos casos, respectivamente.

Para as populações com $N=10$, quando $M=5$ ou $F=0,2$ os preditores empíricos obtidos sob MSS com a constante $\widehat{k}_{i 1}^{(S S)}$ ou $\widehat{k}_{i 2}^{(S S)}$ são respectivamente os melhores ou equivalentes ao melhor em 50\%-90\% ou $80 \%-97 \%$ dos casos, mas quando . $\mathrm{e}$ e $F$ aumentam o preditor empírico com melhor desempenho ou equivalente a ele é aquele obtido sob o MPA em $93 \%-100 \%$ dos casos. Para as populaçoes com $N=50$. este melhor desempenho é atingido somente pelo preditor empírico obtido sob o 
Tabela 3.1: Porcentagem de ocorrências de EQME mínimo para o preditor empírico com melhor desempenho (populações com variâncias intra-grupos diferentes)

\begin{tabular}{|c|c|c|c|c|c|c|c|}
\hline \hline \multirow{2}{*}{\multicolumn{2}{|c|}{ População }} & \multicolumn{5}{|c|}{ Fração de amostragem dos grupos $F$} \\
\cline { 3 - 8 } \multicolumn{2}{|c|}{} & \multicolumn{2}{|c|}{0.2} & \multicolumn{2}{c|}{0.5} & \multicolumn{2}{c|}{0.8} \\
\cline { 3 - 8 } & Preditor & $\%$ & Preditor & $\%$ & Preditor & $\%$ \\
\hline \hline \multirow{4}{*}{ Tipo 1 } & $N=10, M=5$ & $M S S 2^{n}$ & 40 & $M G, M S S 2$ & 40 & $M G, M S S 2$ & 40 \\
& $N=10, M=20$ & $M G$ & 73 & $M G$ & 70 & $M P A$ & 50 \\
& $N=50, M=20$ & $M P A$ & 77 & $M P A$ & 90 & $M P A$ & 90 \\
\hline \multirow{4}{*}{ Tipo 2 } & $N=10, M=5$ & $M G, M S S 2$ & 40 & $M G$ & 60 & $M P A$ & 50 \\
& $N=10, M=20$ & $M G$ & 70 & $M P A$ & 43 & $M S S 1^{b}$ & 43 \\
& $N=50, M=20$ & $M P A$ & 53 & $M P A$ & 43 & $M S S 1$ & 43 \\
\hline \hline
\end{tabular}

${ }^{a} M S S 2$ denota o preditor obtido sob o MSS com a constante $\widehat{k}_{i 2}^{(S S)}$

${ }^{\prime} M S S 1$ denota o preditor obtido sob o MSS com a constante $\widehat{k}_{i 1}^{(S S)}$

MPA em $100 \%$ dos casos.

É importante notar que todos os preditores empíricos, exceto aquele obtido sob o $M P A$, apresentam desempenho ruim para certas situações. Em particular, para populações com $M=5$, o pior desempenho corresponde ao preditor empírico obtido sob o $M L M G$, quando as componentes de variância são estimadas por máxima verossimilhança, em 20\%-70\% dos casos e um ARP máximo global de $294 \%$. Para populações com $M=20$, este desempenho ruim acontece com o preditor empírico obtido sob o $M S S$ com a constante $\widehat{k}_{i 2}^{(S S)}$ em $50 \%-60 \%$ dos casos e um ARP máximo global de $2687 \%$.

\section{Variâncias intra-grupo iguais}

Neste caso, o melhor desempenho também depende das características da população e das frações de amostragem. Na Tabela 3.2 apresentamos os dois preditores empíricos que atingem as maiores porcentagens de ocorrências de EQME mínimo. Quando na Tabela 3.2 citemos o preditor empírico MSS. vamos nos estar referindo ao preditor empírico obtido sob o MSS com a constante de encolhimento $\widehat{h}_{1}^{(S S)}$, pois como era de esperar. o preditor empírico sob o MSS com a constante de encolhimento $\widehat{k}_{2}^{(S S)}$ apresenta o mesmo desempenho que o preditor empírico sob o MPA. 
Adicionalmente, aparecem na Tabela 3.2 porcentagens cuja soma excede a.o $100 \%$ (identificadas com *), por que os preditores empíricos obtidos sob o MSS e sob o MPA apresentam o mesmo EQME.

Tabela 3.2: Porcentagem de ocorrências de EQME mínimo para os dois preditores empíricos com melhor desempenho (populações com variâncias intra-grupos iguais)

\begin{tabular}{|c|l|l|l|l|l|c|}
\hline \hline \multirow{3}{*}{ População } & \multicolumn{5}{|c|}{ Fração de amostragem dos grupos $F$} \\
\cline { 2 - 7 } & \multicolumn{2}{|c|}{0.2} & \multicolumn{2}{c|}{0.5} & \multicolumn{2}{c|}{0.8} \\
\cline { 2 - 7 } & Preditor & $\%$ & Preditor & $\%$ & Preditor & $\%$ \\
\hline \hline \multirow{2}{*}{$N=10 M=5$} & $1) M G$ & 50 & 1) $M G$ & 50 & 1) $M S S$ & $80^{*}$ \\
& 2) $M P A$ & 30 & 2) $M P A$ & 30 & 2) $M P A$ & $60^{*}$ \\
\hline \multirow{2}{*}{$N=10 M=20$} & 1) $M G$ & 40 & 1) $M P A$ & $43^{*}$ & 1) $M S S$ & $73^{*}$ \\
& 2) $M P A$ & $37^{*}$ & 2) $M G$ & 40 & 2) MPA & $63^{*}$ \\
\hline \multirow{2}{*}{$N=50 M=20$} & 1) $M P A$ & $73^{*}$ & 1) $M S S$ & $90^{*}$ & 1) $M S S$ & $80^{*}$ \\
& 2) $M S S$ & $70^{*}$ & 2) $M P A$ & $70^{*}$ & 2) $M P A$ & $60^{*}$ \\
\hline \hline
\end{tabular}

A partir da Tabela 3.2 observamos que para as populações com $N=10$ e fraçōes de amostragem dos grupos pequenas $(F=0,2)$, o melhor preditor empírico é a $M G$ em 40\%-50\% dos casos. Quando $N$ e $F$ aumentam, os dois melhores preditores empíricos são os obtidos sob o MSS ou sob o $M P A$ em $70 \%-90 \%$ ou $60 \%-73 \%$ dos casos, respectivamente.

Os preditores empíricos obtidos sob o MPA ou sob o MSS são os melhores ou equivalentes ao melhor em $90 \%-100 \%$ ou $83 \%-100 \%$ dos casos, respectivamente.

Os preditores empíricos que apresentam desempenho rium para certas situaçōes são a $M G$ e o obtido sob o $M L M G$. Para a $M G$, este desempenho ruim acontece para $\rho=0,1$, atingindo um valor máximo de $145 \%$. Para o preditor empírico obtido sob o $M L M G$, este desempenho inferior geralmente acontece quando $\rho \leq 0.25$ e $f \geq 0.6$. com um valor máximo de $481 \%$. 


\subsection{Discussão e conclusões}

O modelo que fornece os melhores preditores ou preditores empíricos depende das características da população e das frações de amostragem. Em particular, quando $f$ é muito pequena aparecem problemas de convergência na obtenção dos preditores empíricos sob o $M L M G$.

Quando as componentes de variância são conhecidas e as variâncias intra-grupo são diferentes o preditor com menor EQMS é, em geral, aquele obtido sob o MPA quando a estrutura populacional é de tipo 1 ou aquele obtido sob o $M S S$, quando a estrutura é de tipo 2.

Quando as componentes de variância são conhecidas e as variâncias intra-grupo são iguais, o melhor preditor é o obtido sob o $M P A$ quando $N$ e $M$ são pequenos, ou aqueles obtidos sob o $M P A$ ou sob o $M L M G$, quando ambos $N$ e $M$ aumentam.

Quando as variâncias intra-grupo são diferentes e desconhecidas, os melhores preditores empíricos são a média do grupo ou aquele obtido sob o $M S S$ (com $\widehat{k}_{i 2}^{(S S)}$ ) quando $N, M$ e $F$ são pequenos, mas quando esses parâmetros aumentam, os melhores preditores são aqueles obtidos sob o MPA quando a estrutura populacional é de tipo 1 ou os obtidos sob o MPA ou sob o $M S S$ ( $\operatorname{com} \widehat{k}_{i 2}^{(S S)}$ ) quando a estrutura. populacional é de tipo 2 .

Quando as variâncias intra-grupo são iguais e desconhecidas, o melhor preditor empírico é a média do grupo para $F=0,2$ e populações com $N=10$, mas quando $N, M$ ou $F$ aumentam os melhores preditores empíricos são os obtidos sob o MSS ou sob o $M P A$.

Em todos os casos, os preditores cbtidos sob o $M P A$ são os mas estáveis, sendo os melhores ou equivalentes a eles. Além disso, são os únicos que não apresentam desempenho ruim (i.e., ARP no EQME com relação ao EQME do melhor preditor cmpírico superior a $50 \%$ ), diferentemente de seus competidores.

O bom desempenho do preditor obtido sob o MPA era esperado. principalmente no caso de variancias intra-grupos iguais. pois este preditor leva em conta tanto o planejamento quanto a finitude da população fazendo um mínimo de suposiçōes que relacionam numa forma mais natural e direta o modelo probabilístico com a população finita, a diferênça tanto do preditor obtido sob o MSS que, embora desenvolvido para populações finitas, faz suposições relativamente "artificiais", quanto 
daqueles obtidos sob modelos para populações infinitas, em que, se as suposições distribucionais não são satisfeitas, o seu comportamento é imprevisível. 


\section{Capítulo 4}

\section{Estimadores e preditores ótimos em presença de covariáveis}

\subsection{Introdução}

Neste capítulo estudamos problemas de inferência em populações finitas sob amostragem em dois estágios sem reposição, orientando-nos especificamente para a estimação da resposta média (ou total) geral assim como para a predição de valores latentes de grupos em presença de covariáveis. Adicionalmente, generalizamos a proposta de Li (2003) e Li e Stanek (2005) para obter estimadores da resposta média geral ou nos estratos sob amostragem estratificada em presença de várias covariáveis.

No caso de populações infinitas, tipicamente assumimos que os valores das covariáveis são fixos para todas as unidades amostradas e propomos um modelo que relaciona a variável resposta com as covariáveis. Quando se considera uma população finita, em geral assumimos que os valores das covariáveis são conhecidos para todas as unidades que compoem a população (e portanto são conhecidos também. quer seus totais ou suas médias gerais, quer os totais on as médias dos grupos que compoem a população finita). Neste caso, também propomos modelos que relacionam a variável resposta com as covariáveis. Em particular, para uma amostragem em dois estágios sem reposição a partir de uma população finita agrupada. sob o enfoque do modelo de permutação aleatória podemos considerar: sem perda de ge- 
neralidade, que a amostra no primeiro estágio corresponde aos primeiros $n$ grupos numa permutação aleatória e a amostra no segundo estágio corresponde às primeiras $m$ unidades numa permutação aleatória das unidades dentro do grupo. Como resultado, quando avaliamos uma única variável, o vetor cujos elementos são os parâmetros populacionais individuais associados às unidades está relacionado com um vetor de variáveis aleatórias cuja aleatoriedade é induzida pelo planejamento. Quando adicionalmente consideramos uma ou mais covariáveis para cada unidade na população, o vetor com os parâmetros individuais associados a cada unidade na população contém tanto os elementos da resposta quanto das covariáveis. Neste caso, também é a permutação das unidades que induz a aleatoriedade tanto para a resposta quanto para as covariáveis. Assim, não é necessário propor um modelo que relacione a resposta com as covariáveis, ele é consequiência da permutação conjunta da resposta e das variáveis auxiliares. A informação conhecida sobre as covariáveis (como totais ou médias) é incorporada por meio da centralização. Estimadores que sejam funções lineares dos valores amostrados, não viesados e com erro quadrático médio mínimo podem ser obtidos sem necessidade de nenhuma suposição adicional.

\subsection{Amostragem em dois estágios}

\subsubsection{Definições básicas}

Consideremos uma população finita composta por $N$ grupos com $M$ unidades cada um ( $N$ e $M$ conhecidos). Associada com cada unidade $t$ do grupo $s$ na população $(t=1, \ldots, M, s=1, \ldots, N)$ temos um vetor de constantes fixas $\left(y_{s t}, x_{s t}^{(1)}, \ldots, x_{s t}^{(p)}\right)$, em que $y_{s t}$ representa à resposta individual de interesse e $x_{s t}^{(1)}, \ldots, x_{s t}^{(p)}$ representam valores de $p$ covariáveis. Tanto os valores da resposta quanto os das covariáveis são fixos para cada unidade populacional e representam os parâmetros de interesse, denominados parâmetros individuais. Assumimos também que quando observamos a unidade $t$ do grupo $s$, os paràmetros individuais $y_{s t} \mathrm{e}$ $x_{s t}^{(1)}, \ldots, x_{s t}^{(p)}$ são observados sem erro.

Representamos o conjunto das respostas individuais de interesse por meio do vetor $\mathbf{y}=\left(\mathbf{y}_{1}^{\prime}, \ldots, \mathbf{y}_{N}^{\prime}\right)^{\prime}$. em que $\mathbf{y}_{s}=\left(y_{s 1} \ldots, y_{s, M}\right)^{\prime}$ corresponde ao vetor de respostas individuais do grupo s. Análogamente, representamos o conjunto dos 
parâmetros auxiliares individuais mediante a matriz $\mathrm{X}=\left(\mathrm{x}^{(1)}, \ldots, \mathrm{x}^{(p)}\right)$, sendo $\mathrm{x}^{(k)}=\left(\mathrm{x}_{1}^{(k)^{\prime}}, \ldots, \mathrm{x}_{N}^{(k)^{\prime}}\right)^{\prime}, k=1, \ldots, p$, o vetor de parâmetros individuais associados à $k$-ésima variável auxiliar, $\operatorname{com} \mathbf{x}_{s}^{(k)}=\left(x_{s 1}^{(k)}, \ldots, x_{s M}^{(k)}\right)^{\prime}, s=1, \ldots, N$ denotando o vetor dos parâmetros individuais da $k$-ésima variável auxiliar associados às unidades do grupo $s$. Assim, podemos representar a população mediante a matriz não-estocástica $(\mathrm{y}, \mathrm{X})$. Para o grupo $s, s=1, \ldots, N$, definamos:

$\mu_{s}^{(y)}=\frac{1}{M} \sum_{t=1}^{M} y_{s t}=\frac{1}{M} 1_{M}^{\prime} \mathrm{y}_{s}$, a média das respostas nas unidades do grupo $s$,

$\sigma_{s ; y}^{2}=\frac{1}{M-1} \sum_{t=1}^{M}\left(y_{s t}-\mu_{s}^{(y)}\right)^{2}=\frac{1}{M-1} \mathbf{y}_{s}^{\prime} \mathbf{P}_{M} \mathbf{y}_{s}$, a variância das respostas associadas às unidades do grupo $s$, i.e., a variância da resposta dentro do grupo $s$ (sendo $\left.\mathrm{P}_{M}=\mathrm{I}_{M}-M^{-1} \mathbf{J}_{M}\right)$,

$\sigma_{s ; y, k}=\frac{1}{M-1} \sum_{t=1}^{M}\left(y_{s t}-\mu_{s}^{(y)}\right)\left(x_{s t}^{(k)}-\mu_{s}^{(k)}\right)=\frac{1}{M-1} \mathbf{y}_{s}^{\prime} \mathrm{P}_{M} \mathbf{x}_{s}^{(k)}, k=1, \ldots, p, \mathrm{a}$. covariância entre a resposta e a $k$-ésima variável auxiliar nas unidades do grupo $s$,

$\sigma_{e ; y}^{2}=\frac{1}{N} \sum_{s=1}^{N} \sigma_{s ; y}^{2}$, a média das variâncias da resposta dentro dos grupos, e $\sigma_{e ; y, k}=\frac{1}{N} \sum_{s=1}^{N} \sigma_{s: y, k}, k=1, \ldots, p$, a média das covariâncias entre a resposta e a $k$-ésima variável auxiliar dentro dos grupos.

Analogamente, para as covariáveis, podemos definir $\mu_{s}^{(k)}=\frac{1}{M} 1_{M}^{\prime} \mathrm{x}_{s}^{(k)}$, $\sigma_{s ; k}^{2}=\frac{1}{M-1} \mathbf{x}_{s}^{(k)^{\prime}} \mathbf{P}_{M} \mathbf{X}_{s}^{(k)}, \quad \sigma_{s ; k, l}=\frac{1}{M-1} \mathbf{x}_{s}^{(k)^{\prime}} \mathbf{P}_{M} \mathbf{x}_{s}^{(l)}, \quad \sigma_{e ; k}^{2}=\frac{1}{N} \sum_{s=1}^{N} \sigma_{s ; k}^{2} \quad \mathrm{e}$ $\sigma_{e ; k, l}=\frac{1}{N} \sum_{s=1}^{N} \sigma_{s ; k, l}$, para $k, l=1, \ldots, p, k \neq l$.

Assim, $\boldsymbol{\mu}^{(y)}=\left(\mu_{1}^{(y)}, \ldots, \mu_{N}^{(y)}\right)^{\prime}$ e $\mu^{(k)}=\left(\mu_{1}^{(k)}, \ldots, \mu_{N}^{(k)}\right)^{\prime}, k=1, \ldots, p$, representam os vetores das respostas médias e das médias das $p$ covariáveis para os $N$ grupos, respectivamente. Por outro lado, a matriz

$$
\Sigma_{e}=\left(\begin{array}{cc}
\sigma_{\epsilon ; y}^{2} & \sigma_{e: y . X} \\
\sigma_{e: X: y} & \Sigma_{e: X}
\end{array}\right)
$$

contém todas as variâncias e covariâncias médias para a resposta e as p covariáveis. sendo

$$
\sigma_{e: y, Y}=\left(\sigma_{e: y, 1}, \ldots, \sigma_{e: y, p}\right)=\sigma_{e: Y, y}^{\prime}
$$


e

$$
\Sigma_{e ; X}=\left(\begin{array}{cccc}
\sigma_{e ; 1}^{2} & \sigma_{e ; 1,2} & \cdots & \sigma_{e ; 1, p} \\
\sigma_{e ; 2,1} & \sigma_{e ; 2}^{2} & \cdots & \sigma_{e ; 2, p} \\
\vdots & \vdots & \ddots & \vdots \\
\sigma_{e ; p, 1} & \sigma_{e ; p, 2} & \cdots & \sigma_{e ; p}^{2}
\end{array}\right)
$$

Finalmente, definamos:

$\mu^{(y)}=\frac{1}{N} \sum_{s=1}^{N} \mu_{s}^{(y)}=\frac{1}{N} 1_{N}^{\prime} \mu^{(y)}$, a média populacional da resposta, $\sigma_{\mu ; y}^{2}=\frac{1}{N-1} \sum_{s=1}^{N}\left(\mu_{s}^{(y)}-\mu^{(y)}\right)^{2}=\frac{1}{N-1} \mu^{(y)^{\prime}} \mathrm{P}_{N} \mu^{(y)}$, a variância da resposta entre grupos, e

$\sigma_{\mu ; y, k}=\frac{1}{N-1} \sum_{s=1}^{N}\left(\mu_{s}^{(y)}-\mu^{(y)}\right)\left(\mu_{s}^{(k)}-\mu^{(k)}\right)=\frac{1}{N-1} \boldsymbol{\mu}^{(y)^{\prime}} \mathbf{P}_{N} \boldsymbol{\mu}^{(k)}, k=1, \ldots, p$, a covariância entre a resposta e a $k$-ésima variável auxiliar entre grupos.

Similarmente, definamos para as covariáveis os seguintes parâmetros (obtidos como funções dos parâmetros individuais), $\mu^{(k)}=\frac{1}{N} 1_{N}^{\prime} \mu^{(k)}$, $\sigma_{\mu ; k}^{2}=\frac{1}{N-1} \mu^{(k)^{\prime}} \mathbf{P}_{N} \mu^{(k)}$. e $\sigma_{\mu ; k, l}=\frac{1}{N-1} \mu^{(k)^{\prime}} \mathbf{P}_{N} \mu^{(l)}$, para $k, l=1, \ldots, p, k \neq l$.

Desta forma, $\mu=\left(\mu^{(y)}, \mu^{(1)}, \ldots, \mu^{(p)}\right)^{\prime}$ denota um vetor cujos elementos são a resposta média e as médias gerais das $p$ covariáveis e

$$
\Sigma_{\mu}=\left(\begin{array}{cc}
\sigma_{\mu ; y}^{2} & \sigma_{\mu ; y, X} \\
\sigma_{\mu ; X ; y} & \Sigma_{\mu ; X}
\end{array}\right)
$$

contém todas as variàncias e covariâncias entre a resposta e as $p$ covariáveis entre grupos, com

$$
\sigma_{\mu ; y, X}=\left(\sigma_{\mu: y \cdot 1}, \ldots, \sigma_{\mu: y \cdot p}\right)=\sigma_{\mu: X, y}^{\prime}
$$

e

$$
\Sigma_{\mu ; X^{*}}=\left(\begin{array}{cccc}
\sigma_{\mu: 1}^{2} & \sigma_{\mu: 1,2} & \cdots & \sigma_{\mu: 1, p} \\
\sigma_{\mu: 2.1} & \sigma_{\mu: 2}^{2} & \cdots & \sigma_{\mu: 2 . p} \\
\vdots & \vdots & \ddots & \vdots \\
\sigma_{\mu ; p .1} & \sigma_{\mu ; p, 2} & \cdots & \sigma_{\mu: p}^{2}
\end{array}\right)
$$




\subsubsection{Modelo não-estocástico para uma variável resposta e $p$ covariáveis}

Tanto para a resposta quanto para cada uma das $p$ covariáveis, podemos definir modelos lineares não estocásticos para representar a população finita. Eles são dados respectivamente, por:

$$
\mathrm{y}=\left(1_{N} \otimes 1_{M}\right) \mu^{(y)}+\left(\mathrm{I}_{N} \otimes 1_{M}\right) \beta^{(y)}+\epsilon^{(y)}
$$

e

$$
\mathrm{x}^{(k)}=\left(\mathbf{1}_{N} \otimes \mathbf{1}_{M}\right) \mu^{(k)}+\left(\mathbf{I}_{N} \otimes \mathbf{1}_{M}\right) \boldsymbol{\beta}^{(k)}+\epsilon^{(k)}, \quad k=1, \ldots, p
$$

em que

$$
\begin{array}{rlrl}
\beta^{(y)} & =\left(\beta_{1}^{(y)}, \ldots, \beta_{N}^{(y)}\right)^{\prime} \in \mathbb{R}^{N} & \beta_{s}^{(y)} & =\mu_{s}^{(y)}-\mu^{(y)} \\
\beta^{(k)} & =\left(\beta_{1}^{(k)}, \ldots, \beta_{N}^{(k)}\right)^{\prime} \in \mathbb{R}^{N} & & \beta_{s}^{(k)}=\mu_{s}^{(k)}-\mu^{(k)} \\
\epsilon^{(y)} & =\left(e_{11}^{(y)}, \ldots, e_{N M}^{(y)}\right)^{\prime} \in \mathbb{R}^{N M} & \text { com } & e_{s t}^{(y)}=y_{s t}-\mu_{s}^{(y)} \\
\epsilon^{(k)}=\left(e_{11}^{(k)}, \ldots, e_{N M}^{(k)}\right)^{\prime} \in \mathbb{R}^{N M} & e_{s t}^{(k)}=x_{s t}^{(k)}-\mu_{s}^{(k)}
\end{array}
$$

para $k=1, \ldots, p, s=1, \ldots, N$ e $t=1, \ldots, M$. Podemos reunir estas relações no modelo não estocástico

$$
(\mathrm{y}, \mathrm{X})=\left(\mathbf{1}_{N} \otimes 1_{M}\right) \mu^{\prime}+\left(\mathbf{I}_{N} \otimes 1_{M}\right) \boldsymbol{\beta}+\epsilon
$$

em que

$$
\begin{aligned}
& \mu=\left(\mu^{(y)}, \mu^{(X)^{\prime}}\right)^{\prime} \quad \quad \mu^{(X)}=\left(\mu^{(1)}, \ldots, \mu^{(p)}\right)^{\prime} \\
& \beta=\left(\beta^{(y)}, \beta^{(X)}\right) \quad \text { com } \quad \beta^{(X)}=\left(\beta^{(1)}, \ldots, \beta^{(p)}\right) \\
& \epsilon=\left(\epsilon^{(y)}, \epsilon^{\left(X^{\prime}\right)}\right) \quad \epsilon^{\left.()^{\prime}\right)}=\left(\epsilon^{(1)}, \ldots, \epsilon^{(p)}\right)
\end{aligned}
$$

\subsubsection{Modelo estocástico para uma variável resposta e $p$ covariáveis}

Li (2003) e Li e Stanek (2005) obtèm o estimador do total populacional desenvolvendo $u$ modelo de permutação aleatória que, para amostragem aleatória simples, incorpora a informação de uma ou várias covariáveis e para uma amostragem estratificada, só uma covariável. Neste trabalho. desenvolvemos um modelo 
de permutação aleatória que incorpora uma o várias covariáveis tanto para estimar parâmetros quanto para predizer variáveis aleatórias $q$-variados $(q \geq 1)$ sob uma amostragem em dois estágios. O modelo para este tipo de amostragem tem a dificuldade adicional de considerar dois níveis de permutação (de grupos e de unidades dentro de grupos), a diferença daqueles para amostragem aleatória simples ou estratificada que usam só um nível de permutação.

Para uma amostragem em dois estágios, do mesmo modo com que foi desenvolvido o modelo misto de permutação aleatória para uma variável, vamos considerar aqui que a população finita dada por $(\mathrm{y}, \mathrm{X})$ é uma realização da matriz aleatória $(\boldsymbol{Y}, \boldsymbol{X})$ induzida pelo esquema amostral em dois estágios. Consideremos novamente as matrizes $\boldsymbol{U}$ e $\boldsymbol{U}^{(s)}$ de variáveis indicadoras (aleatórias) correspondentes às permutações dos grupos e das unidades dentro dos grupos, respectivamente.

A matriz de variáveis aleatórias $(\boldsymbol{Y}, \boldsymbol{X}) \in \mathbb{R}^{N M \times(p+1)}$, que representa a permutação conjunta da resposta e das variáveis auxiliares pode ser expressa por meio de

$$
\begin{aligned}
& (Y, X)=\left(U \cdot \otimes \mathrm{I}_{M}\right)\left({\underset{s=1}{N}}_{s}^{N} U^{(s)}\right)(\mathrm{y}, \mathrm{X}) \\
& =\left(U \otimes \mathrm{I}_{M}\right)\left(\oplus_{s=1}^{N} U^{(s)}\right)\left[\left(1_{N} \otimes 1_{M}\right) \boldsymbol{\mu}^{\prime}+\left(\mathrm{I}_{N} \otimes 1_{M}\right) \boldsymbol{\beta}+\epsilon\right] \\
& =\left(1_{N} \otimes 1_{M}\right) \mu^{\prime}+\left(\mathrm{I}_{N} \otimes 1_{M}\right) \boldsymbol{U} \boldsymbol{\beta}+\left(\boldsymbol{U} \otimes \mathbf{I}_{M}\right)\left(\bigoplus_{s=1}^{N} U^{(s)}\right) \boldsymbol{\epsilon} \\
& =\left(1_{N} \otimes 1_{M}\right) \mu^{\prime}+\left(\mathrm{I}_{N} \otimes 1_{M}\right) B+E
\end{aligned}
$$

em que

$$
\begin{aligned}
B= & \boldsymbol{U} \beta=\left(\boldsymbol{B}^{(y)}, \boldsymbol{B}^{(X)}\right) \in \mathbb{R}^{N M \times(p+1)} \text { é a matriz dos efeitos aleatórios associados } \\
& \text { à variável resposta e as covariáveis, com } B^{\left(X^{\prime}\right)}=\left(B^{(1)}, \ldots, B^{(p)}\right) \text {, e } \\
E= & \left(\boldsymbol{E}^{(y)}, \boldsymbol{E}^{\left(X^{\prime}\right)}\right), \text { sendo } \boldsymbol{E}^{(X)}=\left(\boldsymbol{E}^{(1)} \ldots, \boldsymbol{E}^{(p)}\right)
\end{aligned}
$$

Alternativamente. este modelo pode ser escrito em uma forma semelhante ao modelo de regressōes aparentemente não relacionadas (Seemingly Unrelated 
Regression - SUR) (Zellner, 1963; Srivasta e Giles, (1987)) como

$$
\begin{aligned}
Z & =\left(\begin{array}{c}
\boldsymbol{Y} \\
\operatorname{vec}(\boldsymbol{X})
\end{array}\right) \\
& =\left(\mathbf{I}_{p+1} \otimes \mathbf{1}_{N} \otimes \mathbf{1}_{M}\right) \boldsymbol{\mu}+\left(\mathbf{I}_{p+1} \otimes \mathbf{I}_{N} \otimes \mathbf{1}_{M}\right)\left(\begin{array}{c}
\boldsymbol{B}^{(y)} \\
\operatorname{vec}\left(\boldsymbol{B}^{(X)}\right)
\end{array}\right)+\operatorname{vec}(\boldsymbol{E}) \\
& =\mathbf{F} \boldsymbol{\mu}+\mathbf{G} \boldsymbol{B}+\boldsymbol{E}
\end{aligned}
$$

em que vec(A) denota um vetor formado empilhando as colunas da matriz A. Podese mostrar (ver Apêndice C) que o vetor de médias e a matriz de covariância de $Z$ são dadas por

$$
E_{\xi_{1} \xi_{2}}(Z)=\left(\mathbf{I}_{p+1} \otimes 1_{N} \otimes 1_{M}\right) \mu
$$

e

$$
\begin{aligned}
\operatorname{Var}_{\xi_{1} \xi_{2}}(Z) & =\Sigma_{\mu} \otimes \mathbf{P}_{N} \otimes \mathbf{J}_{M}+\Sigma_{e} \otimes \mathbf{I}_{N} \otimes \mathbf{P}_{M} \\
& =\Sigma_{e} \otimes \mathbf{I}_{N M}+\left(\boldsymbol{\Sigma}_{\mu}-M^{-1} \Sigma_{e}\right) \otimes \mathbf{I}_{N} \otimes \mathbf{J}_{M}-N^{-1} \Sigma_{\mu} \otimes \mathbf{J}_{N M} \\
& =\mathrm{V}
\end{aligned}
$$

com $\Sigma_{e}, \Sigma_{\mu} \in \mathbb{R}^{(p+1) \times(p+1)}$ dadas por (4.1) e (4.4), respectivamente.

\subsubsection{Estimação}

Nosso interesse recai na estimação (ou predição) de combinações lineares da variável aleatória associada a resposta, i.e., $\boldsymbol{\theta}^{(y)}=\mathrm{L}^{\prime} \operatorname{vec}(\boldsymbol{Z})$, com $\mathrm{L}^{\prime} \in \mathbb{R}^{q \times(p+1) N M}$ uma matriz de constantes conhecidas. Por exemplo, se quisermos estimar a média populacional da resposta, $\mu^{(y)}$, então $\mathrm{L}^{\prime}=(N M)^{-1}\left(1_{N M}^{\prime}, 0_{p N M}^{\prime}\right)$. Por outro lado, se quisermos predizer o vetor dos valores latentes da resposta correspondentes aos grupos realizados, i.e., o vetor das respostas médias dos grupos selecionados na amostragem, basta escolher $\mathrm{L}^{\prime}=\left(\mathrm{I}_{1} \otimes M^{-1} 1_{M}^{\prime}, 0_{n \times p N M}\right)$, com $\mathrm{I}_{1} \bullet=\left(\mathrm{I}_{n}, 0_{n \times(N-n)}\right)$.

No processo de estimação queremos incorporar a informação fornecida pelas covariáveis. Quando a única informação de que dispomos são os valores amostrados, nossa inferência pode se basear no modelo (4.7), que não inclui nenhum conlecimento adicional sobre as covariáveis. Em outros casos, temos informação das covariáveis para todos os indivíduos na população e, consequientemente, também 
conhecemos o vetor das médias gerais das $p$ covariáveis, $\boldsymbol{\mu}^{(X)}$. Isto implica que as componentes $\boldsymbol{X}^{(k)}, k=1, \ldots, p$, do vetor de variáveis aleatórias $\operatorname{vec}(\boldsymbol{X})$ que surge da permutação das variáveis auxiliares, devem satisfazer as restrições $\frac{1}{N M} \sum_{i=1}^{N} \sum_{j=1}^{M} X_{i j}^{(k)}=\mu^{(k)}, k=1, \ldots, p$. Uma forma de incorporar estas restrições no processo de estimação é centrando as covariáveis $\boldsymbol{X}^{(k)}, k=1, \ldots, p$ e obtendo um novo modelo. Este novo modelo é desenvolvido na seção a seguir, tomando como referência o modelo (4.7).

Para obter os estimadores (preditores) $\widehat{\boldsymbol{\theta}}$ de interesse, vamos considerar funções lineares homogêneas dos elementos amostrados, não viciadas para o parâmetro (ou variável aleatória) de interesse (i.e., $E(\widehat{\theta}-\theta)=0$ ) que satisfaçam algum critério de otimalidade. Quando o parâmetro de interesse é univariado, o critério de otimalidade que vamos considerar é que o estimador tenha variância mínima (que é equivalente a considerar, no caso de estimadores não viciados, que tenham erro quadrático médio mínimo). Quando nosso interesse recai na predição de algum vetor de variáveis aleatórias, há vários critérios disponíveis que, essencialmente, são versões escalares obtidas a partir da matriz de covariâncias dos erros preditivos $E(\widehat{\boldsymbol{\theta}}-\boldsymbol{\theta})(\widehat{\boldsymbol{\theta}}-\boldsymbol{\theta})^{\prime} \mathrm{e}$, em muitos casos, constituem critérios equivalentes ${ }^{1}$.

Uma vez incorporadas as restrições associadas ao conhecimento das covariáveis por meio da centralização, a incorporação da condição de ausência de vício gera uma restrição adicional, de modo que o processo de estimação reduz-se a um problema de

\footnotetext{
${ }^{1}$ Seja $\theta \in \mathbb{R}^{n}$ um vetor de parâmetros ou variáveis aleatórias de interesse e definamos como critério de otimalidade o seguinte: "Um estimador (preditor) linear $\widehat{\theta}$ é $R_{2}$-ótimo (ou têm risco mínimo $R_{i}$ ) se $R_{i}(\widehat{\theta}, \theta,.) \leq R_{i}(\tilde{\theta}, \theta,$.$) para todo \theta$, em que $\tilde{\theta}$ é qualquer outro estimador (preditor) linear de $\boldsymbol{\theta}^{\prime \prime}$. A expressão $R_{1}(\widehat{\boldsymbol{\theta}}, \boldsymbol{\theta}, \mathbf{A})=E(\hat{\boldsymbol{\theta}}-\boldsymbol{\theta})^{\prime} \mathbf{A}(\hat{\boldsymbol{\theta}}-\theta)$, com $\mathbf{A}$ uma matriz definida positiva, é conhecida como erro quadrático médio preditivo (Searle, Casella e M[cCulloch, 1992. McCulloch e Searle, 2001) ou função de risco $R_{1}$ (Rao e Toutenburg, 1999), segundo se trate de preditores ou estimadores, respectivamente. Se consideramos $\mathbf{A}=\mathbf{I}$ temos $E(\widehat{\boldsymbol{\theta}}-\boldsymbol{\theta})^{\prime}(\widehat{\boldsymbol{\theta}}-\boldsymbol{\theta})=\operatorname{tr}[E(\hat{\boldsymbol{\theta}}-\boldsymbol{\theta})(\widehat{\boldsymbol{\theta}}-$ $\left.\theta)^{\prime}\right]$. Esta última expressão corresponde, no caso de estimadores, ao critério conhecido como $\mathrm{T}$ otimalidade (Subramani, 1991). A função de risco $R_{2}(\hat{\boldsymbol{\theta}} \cdot \boldsymbol{\theta} . \mathbf{a})=\mathbf{a}^{\prime} E(\hat{\boldsymbol{\theta}}-\boldsymbol{\theta})(\hat{\boldsymbol{\theta}}-\boldsymbol{\theta})^{\prime} \mathbf{a}=E\left[\mathbf{a}^{\prime}(\hat{\boldsymbol{\theta}}-\boldsymbol{\theta})\right]^{2}$. para $a \in \mathbb{R}^{n}$. a $\neq 0$ (Rao e Toutenburg, 1999) corresponde. no caso de estimadores, ao critério conhecido como M-otimalidade (Subramani, 1991) ou, no caso de preditores, ao crro cutadrático médio generalizado (Bolfarine e Zacks. 1992) quando $\mathbf{a}=1$. Se consideramos $\tilde{R}_{1}\left(\hat{\theta}_{0} \boldsymbol{\theta} . \mathbf{A}\right)=$ $E(\widehat{\theta}-\theta)^{\prime} \mathbf{A}(\widehat{\theta}-\theta)$. com A uma matriz não-negativa definida, temos que qualquer $\widehat{\theta} R_{1}$-ótimo é também $\bar{R}_{1}$-ótimo e pode-se mostrar que os critérios usando $\bar{R}_{1}$ e $R_{2}$ são equivalentes. Além disso. qualquuer $\widehat{\theta}$ M-ótimo é T-ótimo e se existe um estimador (preditor) M-ótimo, ele é T-ótimo.
} 
minimização restrita. Neste trabalho vamos focar o caso em que temos informação das covariáveis para todos os indivíduos na população, deixando o caso em que só temos informação das covariáveis após a amostragem para uma pesquisa futura.

\subsubsection{Incorporação da informação adicional das covariáveis}

Seja $\mathbf{P}_{N M}=\mathbf{I}_{N M}-\frac{1}{N M} \mathbf{J}_{N M}$, de forma que $\mathbf{P}_{N M} \boldsymbol{X}^{(k)}=\boldsymbol{X}^{(k)}-\mu^{(k)} \mathbf{1}_{N M}$. Definindo

$$
\mathbf{C}=\left(\begin{array}{cc}
\mathbf{I}_{N M} & \mathbf{0}_{N M \times p N M} \\
\mathbf{0}_{p N M \times N M} & \mathbf{I}_{p} \otimes \mathbf{P}_{N M}
\end{array}\right)
$$

temos

$$
Z^{\star}=\mathrm{C} Z=\left(\begin{array}{c}
Y \\
\boldsymbol{X}^{(1)}-\mu^{(1)} 1_{N M} \\
\vdots \\
\boldsymbol{X}^{(p)}-\mu^{(p)} 1_{N M}
\end{array}\right)
$$

com

$$
E_{\xi_{1} \xi_{2}}\left(Z^{\star}\right)=\mathrm{C} E_{\xi_{1} \xi_{2}}(Z)=\left(\begin{array}{c}
1_{N M} \\
0_{p N M}
\end{array}\right) \mu^{(y)}
$$

e

$$
\operatorname{Var}_{\xi_{1} \xi_{2}}\left(Z^{\star}\right)=\operatorname{Var}_{\xi_{1} \xi_{2}}(Z)
$$

Obtemos então um novo modelo dado por

$$
Z^{\star}=\mathrm{C} Z=\mathrm{CF} \mu+\mathrm{CG} B+\mathrm{C} E=\mathrm{F}^{\star} \mu^{(y)}+\mathrm{G}^{\star} B+E^{\star}
$$

em que:

$$
\mathrm{F}^{\star}=\left(\begin{array}{c}
1_{N M} \\
0_{p, N M}
\end{array}\right) \quad \text { e } \quad \mathrm{G}^{\star}=\left(\begin{array}{cc}
\mathrm{I}_{N} \otimes 1_{M} & 0_{N M \times p N} \\
0_{p, N M \times N} & \mathrm{I}_{p} \otimes \mathrm{P}_{N} \otimes 1_{M}
\end{array}\right)
$$

\subsubsection{Amostragem em dois estágios e especificação de elementos amostrados e não amostrados}

Consideremos um processo de amostragem aleatória com dois estágios sem reposição a partir da população de interesse. No primeiro estágio escollhemos uma amostra de $n$ grupos $(n \leq N)$ e no segundo estágio escolhemos uma amostra de $m$ 
unidades $(m \leq M)$ em cada um dos grupos anteriormente selecionados. Subdividamos o vetor $Z^{\star}$ em duas partes: uma parte amostrada (identificada pelo índice $S$ ) e outra não amostrada (identificada pelo índice $R$ ). Deste modo,

$$
\left(\begin{array}{l}
Z_{S}^{\star} \\
Z_{R}^{\star}
\end{array}\right)=\left(\begin{array}{l}
\mathrm{R}_{1} \\
\mathrm{R}_{2}
\end{array}\right) Z^{\star}
$$

em que

$$
\begin{aligned}
& \mathbf{R}_{i}=\mathbf{I}_{p+1} \otimes \mathbf{S}_{i}, i=1,2 \\
& \mathbf{S}_{1}=\mathbf{I}_{1 \bullet} \otimes \tilde{\mathbf{I}}_{1 \bullet} \in \mathbb{R}^{n m \times N M} \\
& \mathbf{S}_{2}=\left(\begin{array}{l}
\mathbf{I}_{1 \bullet} \otimes \tilde{\mathbf{I}}_{2 \bullet} \\
\mathbf{I}_{2 \bullet} \otimes \mathbf{I}_{M}
\end{array}\right) \in \mathbb{R}^{(N M-n m) \times N M}
\end{aligned}
$$

com

$$
\begin{aligned}
& \mathbf{I}_{1 \bullet}=\left(\mathbf{I}_{n}, \mathbf{0}_{n \times(N-n)}\right) \\
& \mathbf{I}_{2 \bullet}=\left(\mathbf{0}_{(N-n) \times n}, \mathbf{I}_{N-n}\right) \\
& \tilde{\mathbf{I}}_{2 \bullet}=\left(\mathbf{0}_{(M-m) \times m}, \mathbf{I}_{M-m}\right) .
\end{aligned}
$$

A partir dos momentos de $\boldsymbol{Z}^{\star}$, obtemos (ver Apêndice C):

1) $E_{\xi_{1} \xi_{2}}\left[\left(\begin{array}{c}Z_{S}^{\star} \\ Z_{R}^{\star}\end{array}\right)\right]=\left(\begin{array}{c}\mathbf{F}_{S}^{\star} \\ \mathbf{F}_{R}^{\star}\end{array}\right) \mu^{(y)}, \quad$ com $\quad \mathbf{F}_{S}^{\star}=\left(\begin{array}{c}1_{n m} \\ \mathbf{0}_{p n m}\end{array}\right)$ e $\quad \mathbf{F}_{R}^{\star}=\left(\begin{array}{c}1_{N M-n m} \\ 0_{p(N M-n m)}\end{array}\right)$, e

2) $\operatorname{Var}_{\xi_{1} \xi_{2}}\left[\left(\begin{array}{c}Z_{S}^{\star} \\ Z_{R}^{\star}\end{array}\right)\right]=\left(\begin{array}{cc}\mathrm{V}_{S} & \mathrm{~V}_{S R} \\ \mathrm{~V}_{R S} & \mathrm{~V}_{R}\end{array}\right)$, com

$$
\begin{aligned}
& \mathrm{V}_{S}= \Sigma_{\mu} \otimes \\
&=\Sigma_{e} \otimes \mathrm{I}_{n} \otimes \mathrm{I}_{m}+\left(\Sigma_{\mu}-M^{-1} \Sigma_{e}\right) \otimes \mathbf{I}_{n} \otimes \mathrm{J}_{m}-N^{-1} \Sigma_{\mu} \otimes \mathbf{J}_{n} \otimes \mathbf{J}_{m} \\
& \mathrm{~V}_{S R}=\left(\Sigma_{\mu}-M^{-1} \Sigma_{e}\right) \otimes\left(\mathrm{I}_{n} \otimes \mathbf{J}_{m \times(M-m)}, \mathbf{O}_{n m \times(N-n) M}\right) \\
&-N^{-1} \Sigma_{\mu} \otimes \mathbf{J}_{n m \times(N M-n m)} \\
&=\mathrm{V}_{R S}^{\prime}
\end{aligned}
$$

$\mathrm{e}$

$$
\begin{aligned}
\mathrm{V}_{R} & =\Sigma_{e} \otimes \mathrm{I}_{Y M-n n}+\left(\Sigma_{\mu}-M^{-1} \Sigma_{e}\right) \otimes\left(\begin{array}{cc}
\mathbf{I}_{n} \otimes \mathbf{J}_{M-m} & 0_{n(M-m) \times(N-n) M} \\
\mathbf{0}_{(N-n) M \times n(M-m)} & \mathbf{I}_{N-n} \otimes \mathbf{J}_{M}
\end{array}\right) \\
& -N^{-1} \Sigma_{\mu} \otimes \mathbf{J}_{N M-n m}
\end{aligned}
$$




\subsubsection{Estimação do total populacional da resposta usando informação das covariáveis}

Queremos estimar $T^{(y)}=N M \mu^{(y)}=\mathrm{L}^{\prime} Z^{\star} \operatorname{com} \mathrm{L}=\left(\begin{array}{c}1_{N M} \\ 0_{p N M}\end{array}\right)$ ou, equivalentemente, $T^{(y)}=\mathrm{L}_{S}^{\prime} Z_{S}^{\star}+\mathrm{L}_{R}^{\prime} Z_{R}^{\star} \operatorname{com} \mathrm{L}_{S}=\left(\begin{array}{c}1_{n m} \\ 0_{p n m}\end{array}\right)$ e $L_{R}=\left(\begin{array}{c}1_{N M-n m} \\ 0_{p(N M-n m)}\end{array}\right)$, usando a informação adicional fornecida pelas covariáveis.

O estimador $\widehat{T}^{(y)}$ deve ser uma função linear não viesada da amostra. Portanto seja

$$
\widehat{T}^{(y)}=\left(\mathbf{L}_{S}^{\prime}+\mathbf{w}^{\prime}\right) Z_{S}^{\star} \quad \text { com } \quad \mathrm{w} \in \mathbb{R}^{(p+1) n m}
$$

$\mathrm{e}$

$$
E\left(\widehat{T}^{(y)}-T^{(y)}\right)=0, \quad \forall T^{(y)}
$$

Então,

$0=E\left(\widehat{T}^{(y)}-T^{(y)}\right)=E\left(\mathbf{w}^{\prime} Z_{S}^{\star}-\mathbf{L}_{R}^{\prime} Z_{R}^{\star}\right)=\mathbf{w}^{\prime} E\left(Z_{S}^{\star}\right)-\mathrm{L}_{R}^{\prime} E\left(Z_{R}^{\star}\right)=\left(\mathbf{w}^{\prime} \mathbf{F}_{S}^{\star}-\mathbf{L}_{R}^{\prime} \mathbf{F}_{R}^{\star}\right) \mu^{(y)}$.

Para que esta expressão seja válida para todo $\mu^{(y)}$, deve satisfazer $\mathbf{w}^{\prime} \mathrm{F}_{S}^{\star}-\mathbf{L}_{R}^{\prime} \mathrm{F}_{R}^{\star}=0$.

A segunda característica que desejamos é que o estimador $\widehat{T}^{(y)}$ tenha variância mínima. Como

$$
\operatorname{Var}\left(\widehat{T}^{(y)}\right)=\left(\mathbf{L}_{S}^{\prime}+\mathbf{w}^{\prime}\right) \operatorname{Var}_{\xi_{1} \xi_{2}}\left(Z_{S}^{\star}\right)\left(\mathbf{L}_{S}+\mathbf{w}\right)=\mathbf{L}_{S}^{\prime} \mathbf{V}_{S} \mathbf{L}_{S}^{\prime}+2 \mathbf{L}_{S}^{\prime} \mathbf{V}_{S} \mathbf{w}+\mathbf{w}^{\prime} \mathbf{V}_{S} \mathbf{w}
$$

e $\mathrm{L}_{S}^{\prime} \mathrm{V}_{S} \mathrm{~L}_{S}^{\prime}$ não depende de $\mathbf{w}$, nosso objetivo é minimizar

$$
\phi(\mathbf{w}, \lambda)=\mathbf{w}^{\prime} \mathbf{V}_{S} \mathbf{w}+2 \mathbf{L}_{S}^{\prime} \mathbf{V}_{S} \mathbf{w}+2 \lambda\left(\mathbf{w}^{\prime} \mathrm{F}_{S}^{\star}-\mathbf{L}_{R}^{\prime} \mathbf{F}_{R}^{\star}\right)
$$

para $\lambda \in \mathbb{R}$. As derivadas parciais de $\phi(w, \lambda)$ com relação a w e $\lambda$ são dadas por

$$
\begin{aligned}
\frac{\partial \phi}{\partial \mathbf{w}} & =2 \mathbf{V}_{S} \mathbf{w}+2 \mathbf{V}_{S} L_{S}+2 \lambda \mathbf{F}_{S}^{\star} \\
\frac{\partial \phi}{\partial \lambda} & =2\left(\mathbf{w}^{\prime} \mathbf{F}_{S}^{\star}-\mathrm{L}_{R}^{\prime} \mathbf{F}_{R}^{\star}\right)
\end{aligned}
$$

Igualando estas expressões a zero, obtemos o seguinte sistema de equações

$$
\left(\begin{array}{cc}
\mathrm{V}_{S} & \mathrm{~F}_{S}^{+} \\
\mathrm{F}_{S}^{* \prime} & 0
\end{array}\right)\left(\begin{array}{c}
\mathrm{w} \\
\lambda
\end{array}\right)=\left(\begin{array}{c}
-\mathrm{V}_{S} \mathrm{~L}_{S} \\
\mathrm{~L}_{R}^{\prime} \mathrm{F}_{R}^{*}
\end{array}\right)
$$


Para $\mathrm{V}_{S}$ inversível, a solução é dada por

$\widehat{\lambda}=\left(\mathbf{F}_{S}^{\star \prime} \mathbf{V}_{S}^{-1} \mathbf{F}_{S}^{\star}\right)^{-1}\left[-\mathbf{F}_{S}^{\star \prime} \mathbf{V}_{S}^{-1}\left(-\mathrm{V}_{S} \mathbf{L}_{S}\right)-\mathbf{L}_{R}^{\prime} \mathbf{F}_{R}^{\star}\right]=-\left(\mathbf{F}_{S}^{\star \prime} \mathrm{V}_{S}^{-1} \mathbf{F}_{S}^{\star}\right)^{-1}\left(\mathbf{F}_{S}^{\star \prime} \mathbf{L}_{S}+\mathbf{L}_{R}^{\prime} \mathbf{F}_{R}^{\star}\right)$

e

$$
\widehat{\mathbf{w}}=\mathbf{V}_{S}^{-1}\left(-\mathrm{V}_{S} \mathbf{L}_{S}\right)-\mathrm{V}_{S}^{-1} \mathbf{F}_{S}^{\star} \widehat{\lambda}=-\mathbf{L}_{S}+\mathbf{V}_{S}^{-1} \mathbf{F}_{S}^{\star}\left(\mathbf{F}_{S}^{\star \prime} \mathbf{V}_{S}^{-1} \mathbf{F}_{S}^{\star}\right)^{-1}\left(\mathbf{F}_{S}^{\star \prime} \mathbf{L}_{S}+\mathbf{L}_{R}^{\prime} \mathbf{F}_{R}^{\star}\right) .
$$

Subdividamos $\mathbf{V}_{S}=\boldsymbol{\Sigma}_{\mu} \otimes\left(\mathbf{I}_{n}-N^{-1} \mathbf{J}_{n}\right) \otimes \mathbf{J}_{m}+\boldsymbol{\Sigma}_{e} \otimes \mathbf{I}_{n} \otimes\left(\mathbf{I}_{m}-M^{-1} \mathbf{J}_{m}\right)$ da seguinte forma:

$$
\mathrm{V}_{S}=\left(\begin{array}{cc}
\Gamma_{y} & \Gamma_{y X} \\
\Gamma_{X y} & \Gamma_{X}
\end{array}\right)
$$

com

$$
\begin{aligned}
& \Gamma_{y}=\sigma_{\mu ; y}^{2}\left(\mathbf{I}_{n}-N^{-1} \mathbf{J}_{n}\right) \otimes \mathbf{J}_{m}+\sigma_{e ; y}^{2} \mathbf{I}_{n} \otimes\left(\mathbf{I}_{m}-M^{-1} \mathbf{J}_{m}\right) \in \mathbb{R}^{n m \times n m} \\
& \Gamma_{y X}=\sigma_{\mu ; y, X} \otimes\left(\mathbf{I}_{n}-N^{-1} \mathbf{J}_{n}\right) \otimes \mathbf{J}_{m}+\sigma_{e ; y, X} \otimes \mathbf{I}_{n} \otimes\left(\mathbf{I}_{m}-M^{-1} \mathbf{J}_{m}\right) \in \mathbb{R}^{n m \times p n m} \\
& \Gamma_{X y}=\Gamma_{y X}^{\prime},
\end{aligned}
$$

$\mathrm{e}$

$$
\Gamma_{X}=\Sigma_{\mu ; X} \otimes\left(\mathbf{I}_{n}-N^{-1} \mathbf{J}_{n}\right) \otimes \mathbf{J}_{m}+\Sigma_{e ; X} \otimes \mathbf{I}_{n} \otimes\left(\mathbf{I}_{m}-M^{-1} \mathbf{J}_{m}\right) \in \mathbb{R}^{p n m \times p n m}
$$

Desta forma, para $\Gamma_{y}$ e $\Gamma_{X}$ inversíveis

$$
\begin{aligned}
\mathrm{V}_{S}^{-1} & =\left(\begin{array}{cc}
\left(\Gamma_{y}-\Gamma_{X y}^{\prime} \Gamma_{X}^{-1} \Gamma_{X y}\right)^{-1} & -\Gamma_{y}^{-1} \Gamma_{X y}^{\prime}\left(\Gamma_{X}-\Gamma_{X y} \Gamma_{y}^{-1} \Gamma_{X y}^{\prime}\right)^{-1} \\
-\Gamma_{X}^{-1} \Gamma_{X y}\left(\Gamma_{y}-\Gamma_{X y}^{\prime} \Gamma_{X}^{-1} \Gamma_{X y}\right)^{-1} & \left(\Gamma_{X}-\Gamma_{X y} \Gamma_{y}^{-1} \Gamma_{X y}^{\prime}\right)^{-1}
\end{array}\right) \\
& =\left(\begin{array}{ll}
\Psi_{11} & \Psi_{12} \\
\Psi_{21} & \Psi_{22}
\end{array}\right)
\end{aligned}
$$

e portanto

$$
\mathrm{V}_{S}^{-1} \mathrm{~F}_{S}^{*}=\left(\begin{array}{ll}
\Psi_{11} & \Psi_{12} \\
\Psi_{21} & \Psi_{22}
\end{array}\right)\left(\begin{array}{c}
1_{n m} \\
1_{p} \otimes 0_{n m}
\end{array}\right)=\left(\begin{array}{l}
\Psi_{11} 1_{n m} \\
\Psi_{21} 1_{n m}
\end{array}\right)
$$

com $\mathrm{V}_{S}^{-1} \mathrm{~F}_{S}^{\star} \in \mathbb{R}^{(p+1) n m}, \Psi_{11} 1_{n m} \in \mathbb{R}^{n m}$ e $\Psi_{21} 1_{n m} \in \mathbb{R}^{p n m}$. Assim.

$$
\mathrm{F}_{S}^{\star \prime} \mathrm{V}_{S}^{-1} \mathrm{~F}_{S}^{\star}=\left(1_{m m}^{\prime}, 0_{p n m}^{\prime}\right)\left(\begin{array}{l}
\Psi_{11} 1_{n m} \\
\Psi_{21} 1_{n m}
\end{array}\right)=1_{n m}^{\prime} \Psi_{11} 1_{n m} \in \mathbb{R}
$$

e

$$
\mathbf{F}_{S}^{\star \prime} \mathbf{L}_{S}+\mathbf{L}_{R}^{\prime} \mathbf{F}_{R}^{*}=\left(1_{n m}^{\prime}, 0_{p n m}^{\prime}\right)\left(\begin{array}{c}
1_{n m} \\
0_{p n m}
\end{array}\right)+N M-n m=N M
$$


o que implica que

$$
\widehat{T}^{(y)}=\left(\mathrm{L}_{S}^{\prime}+\mathrm{w}^{\prime}\right) Z_{S}^{\star}=\left(\mathrm{F}_{S}^{\star \prime} \mathrm{L}_{S}+\mathrm{L}_{R}^{\prime} \mathrm{F}_{R}^{\star}\right)\left(\mathbf{F}_{S}^{\star \prime} \mathrm{V}_{S}^{-1} \mathbf{F}_{S}^{\star}\right)^{-1} \mathrm{~F}_{S}^{\star \prime} \mathrm{V}_{S}^{-1} Z_{S}^{\star}
$$

Note que $Z_{S}^{\star}$ pode ser expresso como

$$
Z_{S}^{\star}=\left(\begin{array}{c}
Y_{S} \\
v e c\left(X_{S}\right)-\mu^{(X)} \otimes 1_{n m}
\end{array}\right)
$$

em que $Y_{S}$ denota o vetor resposta amostrado e

$$
\operatorname{vec}\left(\boldsymbol{X}_{S}\right)-\boldsymbol{\mu}^{(X)} \otimes 1_{n m}=\left(\begin{array}{c}
\boldsymbol{X}_{S}^{(1)}-\mu^{(1)} 1_{n m} \\
\vdots \\
\boldsymbol{X}_{S}^{(p)}-\mu^{(p)} 1_{n m}
\end{array}\right)
$$

com $\boldsymbol{X}_{S}^{(k)}$ representando o vetor amostrado da $k$-ésima covariável, $k=1, \ldots, p$. Então,

$$
\begin{aligned}
\widehat{T}^{(y)} & =\frac{N M}{1_{n m}^{\prime} \Psi_{11} 1_{n m}}\left(1_{n m}^{\prime} \Psi_{11}^{\prime}, 1_{n m}^{\prime} \Psi_{21}^{\prime}\right) Z_{S}^{\star} \\
& =\frac{N M}{1_{n m}^{\prime} \Psi_{11} 1_{n m}}\left[1_{n m}^{\prime} \Psi_{11} Y_{S}+1_{n m}^{\prime} \Psi_{21}^{\prime}\left(\operatorname{vec}\left(X_{S}\right)-\mu^{(X)} \otimes 1_{n m}\right)\right] \\
& =\frac{N M}{1_{n m}^{\prime} \Psi_{11} \mathbf{1}_{n m}}\left\{1_{n m}^{\prime} \Psi_{11}\left[Y_{S}-\beta_{y}^{\prime}\left(\operatorname{vec}\left(X_{S}\right)-\mu^{(X)} \otimes 1_{n m}\right)\right]\right\}
\end{aligned}
$$

em que $\beta_{y}=\Gamma_{X}^{-1} \Gamma_{X y}$ e

$$
\operatorname{Var}\left(\widehat{T}^{(y)}\right)=\mathbf{L}_{S}^{\prime} \mathbf{V}_{S} \mathbf{L}_{S}+2 \mathbf{L}_{S}^{\prime} \mathbf{V}_{S} \widehat{\mathbf{w}}+\widehat{\mathbf{w}}^{\prime} \mathbf{V}_{S} \widehat{\mathbf{w}} .
$$

Como $\widehat{\mathbf{w}}=-\mathrm{L}_{S}+\mathrm{q}$ com

$$
\mathrm{q}=\mathrm{V}_{S}^{-1} \mathrm{~F}_{S}^{\star}\left(\mathrm{F}_{S}^{\star \prime} \mathrm{V}_{S}^{-1} \mathrm{~F}_{S}^{\star}\right)^{-1}\left(\mathrm{~F}_{S}^{\star \prime} \mathrm{L}_{S}+\mathrm{L}_{R}^{\prime} \mathrm{F}_{R}^{\star}\right)=\frac{N M}{1_{n m}^{\prime} \Psi_{11} 1_{n m}} \mathrm{~V}_{S}^{-1} \mathbf{F}_{S}^{\star}
$$

temos

$$
\begin{aligned}
\operatorname{Var}\left(\widehat{T}^{(y)}\right) & =\mathrm{L}_{S}^{\prime} \mathrm{V}_{S} \mathrm{~L}_{S}-2 \mathrm{~L}_{S}^{\prime} \mathrm{V}_{S} \mathrm{~L}_{S}+2 \mathrm{~L}_{S}^{\prime} \mathrm{V}_{S} \mathbf{q}+\mathbf{L}_{S}^{\prime} \mathrm{V}_{S} \mathrm{~L}_{S}-\mathrm{L}_{S}^{\prime} \mathrm{V}_{S} \mathbf{q}-\mathrm{q}^{\prime} \mathrm{V}_{S} \mathrm{~L}_{S} \\
& +\mathrm{q}^{\prime} \mathbf{V}_{S} \mathbf{q} \\
& =\mathrm{q}^{\prime} \mathrm{V}_{S} \mathbf{q} \\
& =\left(\frac{N M}{1_{n m}^{\prime} \Psi_{11} 1_{n m}}\right)^{2} \mathrm{~F}_{S}^{\star \prime} \mathrm{V}_{S}^{-1} \mathrm{~V}_{S} \mathrm{~V}_{S}^{-1} \mathrm{~F}_{S}^{+} \\
& =\left(\frac{N M}{1_{n m}^{\prime} \Psi_{11} 1_{n m}}\right)^{2}\left(1_{n m}^{\prime} \Psi_{11} 1_{n m}\right) \\
& =\frac{(N M)^{2}}{1_{n m}^{\prime} \Psi_{11} 1_{n m}}
\end{aligned}
$$


A média populacional da resposta é dada por $\mu^{(y)}=\frac{T^{(y)}}{N M}$, de modo que o estimador linear não viesado e de variância mínima de $\mu^{(y)}$ é dado por

$$
\begin{aligned}
\widehat{\mu}^{(y)} & =\left(\mathrm{F}_{S}^{\star \prime} \mathrm{V}_{S}^{-1} \mathrm{~F}_{S}^{\star}\right)^{-1} \mathrm{~F}_{S}^{\star \prime} \mathrm{V}_{S}^{-1} Z_{S}^{\star} \\
& =\frac{1}{1_{n m}^{\prime} \Psi_{11} 1_{n m}}\left\{1_{n m}^{\prime} \Psi_{11}\left[Y_{S}-\beta_{y}^{\prime}\left(\operatorname{vec}\left(X_{S}\right)-\mu^{(X)} \otimes 1_{n m}\right)\right]\right\}
\end{aligned}
$$

com

$$
\operatorname{Var}\left(\widehat{\mu}^{(y)}\right)=\frac{1}{1_{n m}^{\prime} \Psi_{11} 1_{n m}}
$$

Para uma amostragem aleatória simples, Li e Stanek (2005) obtem que o estimador da resposta média populacional é dado por

$$
\widehat{\mu}^{(y)}=\bar{Y}_{S}-\boldsymbol{\beta}_{y}^{\prime}\left(\bar{X}_{S}-\boldsymbol{\mu}^{(X)}\right)
$$

com

$$
\operatorname{Var}\left(\widehat{\mu}^{(y)}\right)=\left(1-\rho_{y X}^{2}\right)(1-f) \frac{\sigma_{y}^{2}}{n}
$$

em que $\bar{Y}_{S}$ é a média amostral da resposta, $\bar{X}_{S}$ é o vetor das médias amostrais das $p$ covariáveis e $\rho_{y_{X}}^{2}$ é o quadrado do coeficiente de correlação múltiplo entre $\boldsymbol{Y}$ e $\boldsymbol{X}$. Note que em (4.16), a estimação da resposta média é uma média ponderada das respostas amostradas "corrigida" ou "ajustada" pelas covariáveis centradas, semelhante a (4.11). Por outro lado, $\Psi_{11}=\left(\Gamma_{y}-\Gamma_{X y}^{\prime} \Gamma_{X}^{-1} \Gamma_{X y}\right)^{-1}=$ $\left[\boldsymbol{\Gamma}_{y}\left(\mathbf{I}_{n m}-\Gamma_{y}^{-1} \Gamma_{X y}^{\prime} \Gamma_{X}^{-1} \Gamma_{X y}\right)\right]^{-1}$, em que $\Gamma_{y}^{-1} \Gamma_{X y}^{\prime} \Gamma_{X}^{-1} \Gamma_{X y}$ é uma generalização do quadrado do coeficiente de correlação múltiplo, de modo que as expressōes para a variância também são semelhantes.

Uma outra forma alternativa de obter estimadores de $T^{(y)}$ e $\mu^{(y)}$ é mediante o estimador por mínimos quadrados ponderados para o modelo de regressōes aparentemente não relacionadas usando somente a parte amostrada (i.e., $Z_{S}^{\star}=\mathbf{F}_{S}^{\star} \mu^{(y)}+\mathbf{G}_{S}^{\star} B+E_{S}^{\star}=\mathbf{F}_{S}^{\star} \mu^{(y)}+\xi_{S}^{\star}$, com $\left.\operatorname{Var}_{\xi_{1} \xi_{2}}\left(Z_{S}^{\star}\right)=\operatorname{Var}_{\xi_{1} \xi_{2}}\left(\xi_{S}^{\star}\right)=\mathrm{V}_{S}\right)$. Esta metodología fornece as mesmas expressões para $\widehat{T}^{(y)}$ e $\widehat{\mu}^{(y)}$.

\subsubsection{Predição dos valores latentes dos grupos amostrados usando informação das covariáveis}

Queremos predizer os valores latentes dos grupos amostrados, i.e. $\theta^{(y)}=\mathrm{L}^{\prime} Z^{\star} \in \mathbb{R}^{n}$ com $\mathrm{L}^{\prime}=\left(\mathrm{I}_{1} \bullet M^{-1} 1_{M}^{\prime}, 0_{n \times p N M}\right)$ e $\mathrm{I}_{1} \bullet=\left(\mathrm{I}_{n} \cdot 0_{n \times(X-n)}\right)$ 
ou, equivalentemente, $\theta^{(y)}=\mathrm{L}_{S}^{\prime} Z_{S}^{\star}+\mathrm{L}_{R}^{\prime} Z_{R}^{\star}$ com

$$
\begin{aligned}
& \mathrm{L}_{S}^{\prime}=\left(\mathbf{I}_{n} \otimes M^{-1} 1_{m}^{\prime}, 0_{n \times p n m}\right) \\
& \mathbf{L}_{R}^{\prime}=\left(\mathbf{I}_{n} \otimes M^{-1} 1_{M-m}^{\prime}, 0_{n \times(N-n) M}, 0_{n \times p(N M-n m)}\right)
\end{aligned}
$$

incorporando a informação adicional fornecida pelas covariáveis.

Procuramos um preditor $\widehat{\theta}^{(y)}$ que seja uma função linear homogênea dos valores amostrados e não viesada para $\theta^{(y)}$, i.e.,

$$
\widehat{\boldsymbol{\theta}}^{(y)}=\mathrm{L}_{S}^{\prime} Z_{S}^{\star}+\mathrm{W}^{\prime} Z_{S}^{\star} \quad \text { com } \quad \mathrm{W}^{\prime} \in \mathbb{R}^{n \times(p+1) n m}
$$

e

$$
E\left(\widehat{\theta}^{(y)}-\theta^{(y)}\right)=0, \forall \theta^{(y)} .
$$

Então,

$$
0=E\left(\widehat{\theta}^{(y)}-\theta^{(y)}\right)=E\left(\mathbf{W}^{\prime} Z_{S}^{\star}-\mathbf{L}_{R}^{\prime} Z_{R}^{\star}\right)=\left(\mathbf{W}^{\prime} \mathbf{F}_{S}^{\star}-\mathbf{L}_{R}^{\prime} \mathbf{F}_{R}^{\star}\right) \mu^{(y)}, \forall \mu^{(y)},
$$

o que gera a restrição $\mathrm{W}^{\prime} \mathrm{F}_{S}^{\star}-\mathrm{L}_{R}^{\prime} \mathrm{F}_{R}^{\star}=0$.

Consideramos como critério de otimalidade minimizar o erro quadrático médio preditivo ou, equivalentemente, o traço da matriz de covariâncias dos erros preditivos (T-otimalidade). Como

$\operatorname{tr}\left[E\left(\hat{\boldsymbol{\theta}}^{(y)}-\boldsymbol{\theta}^{(y)}\right)\left(\hat{\boldsymbol{\theta}}^{(y)}-\boldsymbol{\theta}^{(y)}\right)^{\prime}\right]=\operatorname{tr}\left(\mathrm{W}^{\prime} \mathrm{V}_{S} \mathrm{~W}-\mathrm{L}_{R}^{\prime} \mathrm{V}_{R S} \mathrm{~W}-\mathrm{W}^{\prime} \mathrm{V}_{S R} \mathrm{~L}_{R}+\mathrm{L}_{R}^{\prime} \mathrm{V}_{R} \mathrm{~L}_{R}\right)$ e $\operatorname{tr}\left(\mathbf{L}_{R}^{\prime} \mathrm{V}_{R} \mathbf{L}_{R}\right)$ não depende de $\mathbf{W}$, nosso objetivo é minimizar

$$
\phi(\mathrm{W}, \lambda)=\operatorname{tr}\left(\mathrm{W}^{\prime} \mathrm{V}_{S} \mathrm{~W}-\mathrm{L}_{R}^{\prime} \mathrm{V}_{R S} \mathrm{~W}-\mathrm{W}^{\prime} \mathrm{V}_{S R} \mathrm{~L}_{R}\right)+2 \lambda^{\prime}\left(\mathrm{W}^{\prime} \mathrm{F}_{S}^{\star}-\mathrm{L}_{R}^{\prime} \mathrm{F}_{R}^{\star}\right)
$$

para $\lambda \in \mathbb{R}^{n}$. As derivadas parciais de $\phi(\mathbf{W}, \lambda)$ com relação a $\mathbf{W}$ e $\lambda$ são dadas por (Seber, 1984)

$$
\begin{aligned}
\frac{\partial \phi}{\partial W} & =2 \mathrm{~V}_{S} \mathrm{~W}-2 \mathrm{~V}_{S R} \mathrm{~L}_{R}+2 \mathrm{~F}_{S}^{\star} \lambda^{\prime} \\
\frac{\partial \phi}{\partial \lambda} & =2\left(\mathbf{W}^{\prime} \mathbf{F}_{S}^{\star}-\mathbf{L}_{R}^{\prime} \mathbf{F}_{R}^{\star}\right)
\end{aligned}
$$

Igualando estas expressões a zero, obtemos o seguinte sistema de equações

$$
\left(\begin{array}{cc}
\mathrm{V}_{S} & \mathrm{~F}_{S}^{\star} \\
\mathrm{F}_{S}^{* \prime} & 0
\end{array}\right)\left(\begin{array}{c}
\mathrm{W} \\
\lambda^{\prime}
\end{array}\right)=\left(\begin{array}{c}
-\mathrm{V}_{S R} \mathrm{~L}_{R} \\
\mathrm{~F}_{R}^{* \prime} \mathrm{L}_{R}
\end{array}\right) .
$$


Para $\mathrm{V}_{S}$ inversível, a solução é dada por

$$
\widehat{\lambda^{\prime}}=\left(\mathbf{F}_{S}^{\star \prime} \mathrm{V}_{S}^{-1} \mathbf{F}_{S}^{\star}\right)^{-1}\left(\mathbf{F}_{R}^{\star \prime} \mathbf{L}_{R}-\mathrm{F}_{S}^{\star \prime} \mathrm{V}_{S}^{-1} \mathrm{~V}_{S R} \mathrm{~L}_{R}\right)
$$

e

$$
\widehat{\mathrm{W}^{\prime}}=\left[\mathrm{L}_{R}^{\prime}\left(\mathbf{F}_{R}^{\star}-\mathrm{V}_{R S} \mathrm{~V}_{S}^{-1} \mathbf{F}_{S}^{\star}\right)\left(\mathbf{F}_{S}^{\star \prime} \mathrm{V}_{S}^{-1} \mathbf{F}_{S}^{\star}\right)^{-1}\left(\mathbf{F}_{S}^{\star \prime}+\mathbf{L}_{R}^{\prime} \mathrm{V}_{R S}\right)\right] \mathbf{V}_{S}^{-1}
$$

de modo que

$$
\begin{aligned}
\widehat{\theta}^{(y)} & =\mathrm{L}_{S}^{\prime} Z_{S}^{\star}+\widehat{\mathrm{W}}^{\prime} Z_{S}^{\star} \\
& =\mathrm{L}_{S}^{\prime} Z_{S}^{\star}+\mathrm{L}_{R}^{\prime}\left[\left(\mathrm{F}_{R}^{\star}-\mathrm{V}_{R S} \mathrm{~V}_{S}^{-1} \mathrm{~F}_{S}^{\star}\right)\left(\mathbf{F}_{S}^{\star \prime} \mathrm{V}_{S}^{-1} \mathbf{F}_{S}^{\star}\right)^{-1} \mathbf{F}_{S}^{\star \prime} \mathrm{V}_{S}^{-1} Z_{S}^{\star}\right]+\mathrm{L}_{R}^{\prime} \mathrm{V}_{R S} \mathrm{~V}_{S}^{-1} Z_{S}^{\star} \\
& =\mathrm{L}_{S}^{\prime} Z_{S}^{\star}+\mathbf{L}_{R}^{\prime}\left[\mathrm{F}_{R}^{\star} \widehat{\mu}^{(y)}+\mathrm{V}_{R S} \mathrm{~V}_{S}^{-1}\left(Z_{S}^{\star}-\mathbf{F}_{S}^{\star} \widehat{\mu}^{(y)}\right)\right]
\end{aligned}
$$

em que $\widehat{\mu}^{(y)}=\left(\mathbf{F}_{S}^{\star \prime} \mathbf{V}_{S}^{-1} \mathbf{F}_{S}^{\star}\right)^{-1} \mathbf{F}_{S}^{\star \prime} \mathbf{V}_{S}^{-1} Z_{S}^{\star}$.

Este preditor é o preditor M-ótimo sugerido no Teorema 2.1 de Lencina (2002).

Calculando cada componente de $\widehat{\theta}^{(y)}$ (ver seção C.1.4 no Apêndice C), obtemos

$$
\begin{aligned}
\widehat{\boldsymbol{\theta}}^{(y)} & =f \bar{Y}_{S}+(1-f) \widehat{\mu}^{(y)} \mathbf{1}_{n} \\
& +(1-f)\left[\mathrm{B}_{11} \Psi_{11}+\mathrm{B}_{12} \Psi_{21}\right]\left(Y_{S}-\widehat{\mu}^{(y)} \mathbf{1}_{n m}\right) \\
& +(1-f)\left[\mathrm{B}_{11} \Psi_{12}+\mathrm{B}_{12} \Psi_{22}\right]\left(\operatorname{vec}\left(X_{S}\right)-\mu^{(X)} \otimes \mathbf{1}_{n m}\right)
\end{aligned}
$$

em que

$$
\begin{gathered}
\mathbf{B}_{11}=\left(\sigma_{y}^{* 2} \mathbf{I}_{n}-N^{-1} \sigma_{\mu ; y}^{2} \mathbf{J}_{n}\right) \otimes 1_{m}^{\prime}, \\
\mathbf{B}_{12}=\left(\sigma_{y, X}^{*} \otimes \mathbf{I}_{n}-N^{-1} \sigma_{\mu ; y, X} \otimes \mathbf{J}_{n}\right) \otimes \mathbf{1}_{m}^{\prime}, \\
\bar{Y}_{S}=\left(\mathbf{I}_{n} \otimes \frac{1}{m} \mathbf{1}_{m}^{\prime}\right) Y_{S}, \\
\sigma_{y}^{* 2}=\sigma_{\mu: y}^{2}-\frac{1}{M} \sigma_{e: y}^{2} .
\end{gathered}
$$

e

$$
\sigma_{y, X}^{*}=\sigma_{\mu: y, X}-\frac{1}{M} \sigma_{\epsilon: y, X}
$$

Lencina (2002) e Stanek e Singer (2004) mostram que, em ausência de covariáveis e erro na resposta, o preditor do vetor de valores latentes de grupos amostrados é dado por

$$
\widehat{\theta}^{(y)}=f \bar{Y}_{S}+(1-f) \widehat{\mu}^{(y)} 1_{n}+(1-f) k\left(\bar{Y}_{S}-\widehat{\mu}^{(y)} 1_{n}\right)
$$

com $k=\frac{m \sigma^{* 2}}{m \sigma^{2}+\sigma_{t}^{2}}$. A expressão (4.12) é semellante a esta última, mas incorpora un termo que "ajusta" o preditor em termos das covariáveis centradas. Em ambos 
os casos, a fração de amostragem das unidades amostradas e não amostradas é incorporada aos termos correspondentes no preditor.

Após algumas manipulações algébricas, mostramos que a matriz de covariâncias dos erros preditivos é

$$
\begin{aligned}
E(\widehat{\theta}-\theta)(\widehat{\theta}-\theta)^{\prime} & =\mathrm{L}_{R}^{\prime}\left(\mathrm{V}_{R}-\mathrm{V}_{R S} \mathrm{~V}_{S}^{-1} \mathrm{~V}_{S R}\right) \mathrm{L}_{R} \\
& +\mathbf{L}_{R}^{\prime}\left(\mathbf{F}_{R}^{\star}-\mathrm{V}_{R S} \mathrm{~V}_{S}^{-1} \mathbf{F}_{S}^{\star}\right)\left(\mathbf{F}_{S}^{\star \prime} \mathrm{V}_{S}^{-1} \mathbf{F}_{S}^{\star}\right)^{-1}\left(\mathbf{F}_{R}^{\star}-\mathrm{V}_{R S} \mathrm{~V}_{S}^{-1} \mathbf{F}_{S}^{\star}\right)^{\prime} \mathbf{L}_{R} \\
& =(1-f)\left[\frac{\sigma_{e ; y}^{2}}{M} \mathbf{I}_{n}+(1-f) \sigma_{y}^{* 2} \mathrm{I}_{n}-(1-f) \frac{\sigma_{\mu ; y}^{2}}{N}\right] \\
& +\mathbf{A}_{11} \Psi_{11} \mathbf{A}_{11}^{\prime}+\mathbf{A}_{11} \Psi_{12} \mathrm{~A}_{12}^{\prime}+\mathrm{A}_{12} \Psi_{21} \mathrm{~A}_{11}^{\prime}+\mathrm{A}_{12} \Psi_{22} \mathrm{~A}_{12}^{\prime} \\
& +\frac{1}{\mathbf{1}_{n m}^{\prime} \Psi_{11} \mathbf{1}_{n m}}\left[(1-f) \mathbf{1}_{n}-\mathbf{A}_{11} \Psi_{11} \mathbf{1}_{n m}-\mathrm{A}_{12} \Psi_{12} \mathbf{1}_{n m}\right] \\
& {\left[(1-f) \mathbf{1}_{n}-\mathbf{A}_{11} \Psi_{11} \mathbf{1}_{n m}-\mathbf{A}_{12} \Psi_{12} 1_{n m}\right]^{\prime} }
\end{aligned}
$$

com $A_{11}$ e $A_{12}$ definidas em (C.5) e (C.6), respectivamente e $\Psi_{11}, \Psi_{12}, \Psi_{21}$ e $\Psi_{22}$ definidas em (4.9).

\subsubsection{Estimadores e preditores empíricos usando informação das covariáveis}

A dificuldade na utilização dos estimadores e preditores desenvolvidos nas duas seções prévias é que, na prática, as componentes de variância e covariância nas matrizes $\Sigma_{\mu}$ e $\Sigma_{e}$ são desconhecidas. Pelo menos, a variância para a resposta e as covariâncias dela com as covariáveis em geral não estão disponíveis. É necessário estimar estas componentes para poder construir preditores empíricos, que podem não ser ótimos, mas deixamos para pesquisas futuras avaliar a perda ao fazer esta mudança.

Sänndal et al. (1992) sugerem estimar tanto as variâncias e covariâncias associadlas à resposta quanto aquelas associadas as covariáveis. Deste modo, podemos considerar dois tipos de preditores empíricos: aqueles que usam a informação populacional (conhecida) fornecida pelas covariáveis ou aqueles que não a usam.

A partir das expressōes populacionais, podemos obter as versões amostrais destas 
componentes como:

$$
\begin{aligned}
\tilde{\sigma}_{\mu ; y}^{2} & =\frac{1}{m(n-1)} \boldsymbol{Y}_{S}^{\prime}\left(\mathbf{P}_{n} \otimes \mathbf{J}_{m}\right) \boldsymbol{Y}_{S} \\
\tilde{\sigma}_{\mu ; y, k} & =\frac{1}{m(n-1)} \boldsymbol{Y}_{S}^{\prime}\left(\mathbf{P}_{n} \otimes \mathbf{J}_{m}\right) \boldsymbol{X}_{S}^{(k)} \quad k=1, \ldots, p \\
\widehat{\sigma}_{e ; y}^{2} & =\frac{1}{n(m-1)} \boldsymbol{Y}_{S}^{\prime}\left(\mathbf{I}_{n} \otimes \mathbf{P}_{m}\right) \boldsymbol{Y}_{S} \\
\widehat{\sigma}_{e ; y, k} & =\frac{1}{m(n-1)} \boldsymbol{Y}_{S}^{\prime}\left(\mathbf{I}_{n} \otimes \mathbf{P}_{m}\right) \boldsymbol{X}_{S}^{(k)} \quad k=1, \ldots, p
\end{aligned}
$$

e se não fizermos uso da informação (populacional) das covariáveis

$$
\begin{aligned}
& \tilde{\sigma}_{\mu ; k, l}=\frac{1}{m(n-1)} \boldsymbol{X}_{S}^{(k)^{\prime}}\left(\mathbf{P}_{n} \otimes \mathbf{J}_{m}\right) \boldsymbol{X}_{S}^{(l)} \\
& \widehat{\sigma}_{e ; k, l}=\frac{1}{m(n-1)} \boldsymbol{X}_{S}^{(k)^{\prime}}\left(\mathbf{I}_{n} \otimes \mathbf{P}_{m}\right) \boldsymbol{X}_{S}^{(l)}
\end{aligned}
$$

para $k, l=1, \ldots, p$. É possível mostrar que sob o modelo de permutação aleatória $E\left(\widehat{\sigma}_{e ; y}^{2}\right)=\sigma_{e ; y}^{2}$ e $E\left(\tilde{\sigma}_{\mu ; y}^{2}\right)=m \sigma_{\mu ; y}^{2}+(1-f) \sigma_{e ; y}^{2}$. Portanto, podemos obter estimadores de $\sigma_{e ; y}^{2}$ e $\sigma_{\mu ; y}^{2}$ pelo método dos momentos, nomeadamente

$$
\widehat{\sigma}_{e ; y}^{2}=\frac{1}{n(m-1)} Y_{S}^{\prime}\left(\mathbf{I}_{n} \otimes \mathbf{P}_{m}\right) Y_{S}
$$

$\mathrm{e}$

$$
\widehat{\sigma}_{\mu ; y}^{2}= \begin{cases}0 & \text { se } \tilde{\sigma}_{\mu: y}^{2} \leq(1-f) \widehat{\sigma}_{e ; y}^{2} \\ \frac{1}{m}\left[\tilde{\sigma}_{\mu ; y}^{2}-(1-f) \widehat{\sigma}_{e ; y}^{2}\right] & \text { em caso contrário }\end{cases}
$$

respectivamente.

Do mesmo modo, estimadores de $\sigma_{e: y, k}$ e $\sigma_{\mu: y, k}$ pelo método dos momentos são

$$
\widehat{\sigma}_{e: y, k}=\frac{1}{m(n-1)} Y_{S}^{\prime}\left(\mathbf{I}_{n} \otimes \mathbf{P}_{m}\right) X_{S}^{(k)}
$$

$\mathrm{e}$

$$
\widehat{\sigma}_{\mu: y, k}=\frac{1}{m}\left[\tilde{\sigma}_{\mu: y, k}-(1-f) \widehat{\sigma}_{c: y, k}\right]
$$

respectivamente.

Substituindo estas componentes nas matrizes correspondentes podemos obter os estimadores ou preditores segundo o procedimento detalhado nas duas secoees prévias. 


\subsection{Amostragem estratificada}

Analogamente ao caso de amostragem em dois estágios, para a amostragem estratificada, vamos começar com algumas definições básicas e, a partir de um modelo não-estocástico, obter o modelo estocástico que representa este tipo de amostragem. Para a estimação, vamos primeiramente incorporar o conhecimento das médias das covariáveis nos diferentes estratos por meio da centralização e vamos subdividir o vetor aleatório que representa a permutação conjunta da resposta e das covariáveis em uma parte amostrada e outra não amostrada. Com essa partição, vamos obter os estimadores ótimos da resposta total (ou média) em cada estrato ou na população. Dessa forma, estendemos os resultados de Li (2003) para uma amostragem estratificada, a situações em que o parâmetro é $q$-variado $(q \geq 1)$ e incorporamos várias covariáveis.

\subsubsection{Definições básicas}

Consideremos uma população finita composta por $N$ estratos com $M_{s}$ unidades cada um, $s=1, \ldots, \dot{N}$ ( $N$ e $M_{s}$ conhecidos). Associada com cada unidade $t$ do estrato $s$ na população $\left(s=1, \ldots, N, t=1, \ldots, M_{s}\right)$ temos um vetor de constantes fixas $\left(y_{s t}, x_{s t}^{(1)}, \ldots, x_{s t}^{(p)}\right)$, em que $y_{s t}$ representa à resposta individual de interesse e $x_{s t}^{(1)}, \ldots, x_{s t}^{(p)}$ representam os valores de $p$ covariáveis. Tanto os valores da resposta quanto os das covariáveis são fixos para cada unidade populacional e representam os parâmetros de interesse, denominados parâmetros individuais. Assumimos também que quando observamos a unidade $t$ do estrato $s$, os parâmetros individuais $y_{s t} \mathrm{e}$ $x_{s t}^{(1)}, \ldots, x_{s t}^{(p)}$ são observados sem erro.

Representamos o conjunto das respostas individuais de interesse por meio do vetor $\mathbf{y}=\left(\mathbf{y}_{1}^{\prime}, \ldots, \mathbf{y}_{N}^{\prime}\right)^{\prime}$, em que $\mathbf{y}_{s}=\left(y_{s 1}, \ldots, y_{s, I_{s}}\right)^{\prime}$ corresponde ao vetor de respostas individuais do estrato $s$. Análogamente, representamos o conjunto dos parâmetros auxiliares individuais associados à $k$-ésima variável auxiliar por meio do vetor $\mathbf{x}^{(k)}=$ $\left(\mathrm{x}_{1}^{(k)^{\prime}}, \ldots, \mathrm{x}_{N}^{(k)^{\prime}}\right)^{\prime}, k=1, \ldots, p, \operatorname{com} \mathrm{x}_{s}^{(k)}=\left(x_{s 1}^{(k)}, \ldots x_{s . M_{s}}^{(k)}\right)^{\prime}, s=1, \ldots N$, denotando o vetor dos parâmetros individuais da $k$-ésima variável auxiliar associados às unidades do estrato $s$.

Para o estrato $s, s=1, \ldots, N$, definamos: 
$\mu_{s}^{(y)}=\frac{1}{M_{s}} \sum_{t=1}^{M_{s}} y_{s t}=\frac{1}{M M_{s}} 1_{M_{s}}^{\prime} \mathrm{y}_{s}$, a média das respostas nas unidades do estrato, $\sigma_{s ; y}^{2}=\frac{1}{M_{s}-1} \sum_{t=1}^{M_{s}}\left(y_{s t}-\mu_{s}^{(y)}\right)^{2}=\frac{1}{M_{s}-1} \mathbf{y}_{s}^{\prime} \mathbf{P}_{M_{s}} \mathbf{y}_{s}$, a variância das respostas associadas às unidades do estrato, i.e., a variância da resposta dentro do estrato,

$\sigma_{s ; y, k}=\frac{1}{M_{s}-1} \sum_{t=1}^{M_{s}}\left(y_{s t}-\mu_{s}^{(y)}\right)\left(x_{s t}^{(k)}-\mu_{s}^{(k)}\right)=\frac{1}{M_{s}-1} \mathbf{y}_{s}^{\prime} \mathbf{P}_{M_{s}} \mathbf{x}_{s}^{(k)}, k=1, \ldots, p, \mathrm{a}$ covariância entre a resposta e a $k$-ésima variável auxiliar nas unidades do estrato.

Analogamente, para as covariáveis, podemos definir $\mu_{s}^{(k)}=\frac{1}{M_{s}} 1_{M_{s}}^{\prime} \mathrm{x}_{s}^{(k)}$, $\sigma_{s ; k}^{2}=\frac{1}{M_{s}-1} \mathbf{x}_{s}^{(k)^{\prime}} \mathbf{P}_{M M_{s}} \mathbf{x}_{s}^{(k)}$ e $\sigma_{s ; k, l}=\frac{1}{M_{s}-1} \mathbf{x}_{s}^{(k)^{\prime}} \mathbf{P}_{M_{s}} \mathbf{x}_{s}^{(l)}, \quad$ para $k, l=1, \ldots, p$, $k \neq l$.

Assim, $\mu_{s}=\left(\mu_{s}^{(y)}, \mu_{s}^{(1)}, \ldots, \mu_{s}^{(p)}\right)^{\prime}$ representa o vetor cujos elementos são a resposta média e as médias das $p$ covariáveis no estrato $s$ e

$$
\Sigma_{s}=\left(\begin{array}{cc}
\sigma_{s ; y}^{2} & \sigma_{s ; y, X} \\
\sigma_{s ; X ; y} & \Sigma_{s ; X}
\end{array}\right)
$$

contém todas as variâncias e covariâncias para a resposta e as $p$ covariáveis no estrato $s$, sendo

$$
\sigma_{s ; y, X}=\left(\sigma_{s ; y, 1}, \ldots, \sigma_{s ; y, p}\right)=\sigma_{s ; X, y}^{\prime}
$$

e

$$
\Sigma_{s ; X}=\left(\begin{array}{cccc}
\sigma_{s ; 1}^{2} & \sigma_{s ; 1,2} & \cdots & \sigma_{s ; 1, p} \\
\sigma_{s: 2,1} & \sigma_{s ; 2}^{2} & \cdots & \sigma_{s ; 2, p} \\
\vdots & \vdots & \ddots & \vdots \\
\sigma_{s ; p, 1} & \sigma_{s ; p, 2} & \cdots & \sigma_{s ; p}^{2}
\end{array}\right)
$$

\subsubsection{Modelo não-estocástico para uma variável resposta e $p$ covariáveis}

Tanto para a resposta quanto para cada uma das $p$ covariáveis, podemos definir modelos lineares não estocásticos para representar cada estrato da população finita. Eles são dados respectivamente, por:

$$
\mathrm{y}_{s}=1_{M_{s}} \mu_{s}^{(y)}+\epsilon_{s}^{(y)}
$$


$\mathrm{e}$

$$
\mathbf{x}_{s}^{(k)}=1_{M_{s}} \mu_{s}^{(k)}+\epsilon_{s}^{(k)}, \quad k=1, \ldots, p
$$

em que

$$
\begin{array}{lll}
\epsilon_{s}^{(y)}=\left(e_{s 1}^{(y)}, \ldots, e_{s M_{s}}^{(y)}\right)^{\prime} \in \mathbb{R}^{M_{s}} & & e_{s t}^{(y)}=y_{s t}-\mu_{s}^{(y)} \\
\epsilon_{s}^{(k)}=\left(e_{s 1}^{(k)}, \ldots, e_{s M_{s}}^{(k)}\right)^{\prime} \in \mathbb{R}^{M_{s}} & \text { com } & e_{s t}^{(k)}=x_{s t}^{(k)}-\mu_{s}^{(k)}
\end{array}
$$

para $k=1, \ldots, p, s=1, \ldots, N$ e $t=1, \ldots, M_{s}$. Podemos reunir estas relações no modelo não estocástico

$$
\mathrm{z}_{s}=\left(\mathrm{y}_{s}^{\prime}, \mathrm{x}_{s}^{(1)^{\prime}}, \ldots, \mathrm{x}_{s}^{(p)^{\prime}}\right)^{\prime}=\left(\mathrm{I}_{p+1} \otimes 1_{M_{s}}\right) \mu_{s}+\epsilon
$$

em que

$$
\epsilon=\left(\epsilon_{s}^{(y)^{\prime}}, \epsilon_{s}^{(1)^{\prime}}, \ldots, \epsilon_{s}^{(p)^{\prime}}\right)^{\prime}
$$

\subsubsection{Modelo estocástico para uma variável resposta e $p$ covariáveis}

Para obter o modelo de permutação aleatória induzido pelo esquema amostral estratificado, vamos considerar primeiramente que, para o estrato $s, s=1, \ldots, N$, o vetor $\mathbf{z}_{s}$ é uma realização do vetor aleatório $Z_{s}$ dado por

$$
Z_{s}=\left(\mathrm{I}_{p+1} \otimes U^{(s)}\right) \mathrm{z}_{s}
$$

em que a matriz $U^{(s)} \in \mathbb{R}^{M_{s} \times M_{s}}$ de variáveis indicadoras (aleatórias) corresponde às permutações das unidades dentro do estrato $s$.

Pode-se mostrar que (ver seção C.2.1 no Apèndice C)

$$
E_{\xi_{1}}\left(Z_{s}\right)=\left(\mathbf{I}_{p+1} \otimes 1_{M I_{s}}\right) \mu_{s}
$$

e

$$
\operatorname{Var}_{\xi_{1}}\left(Z_{s}\right)=\Sigma_{s} \otimes \mathrm{P}_{M_{s}}
$$

em que o subindice $\xi_{1}$ indica esperança com relação à permutação das unidades no estrato $s, s=1, \ldots, N$. Deste modo, o retor de variáveis aleatórias $Z=\left(Z_{1}^{\prime}, \ldots, Z_{N}^{\prime}\right)^{\prime} \in \mathbb{R}^{(p+1) . M}\left(\operatorname{com} M=\sum_{s=1}^{N} M_{s}\right)$ que representa a permutação 
conjunta da resposta e das variáveis auxiliares para os $N$ estratos, pode ser expresso por meio de

$$
\begin{aligned}
Z & =\left[\bigoplus_{s=1}^{N}\left(\mathrm{I}_{p+1} \otimes U^{(s)}\right)\right] \mathrm{Z} \\
& =\mathbf{F} \boldsymbol{\mu}+\boldsymbol{E}
\end{aligned}
$$

em que

$$
\begin{aligned}
\mathbf{z} & =\left(\mathbf{z}_{1}^{\prime}, \ldots, \mathbf{z}_{N}^{\prime}\right)^{\prime} \in \mathbb{R}^{(p+1) M} \\
\boldsymbol{\mu} & =\left(\boldsymbol{\mu}_{1}^{\prime}, \ldots, \boldsymbol{\mu}_{N}^{\prime}\right)^{\prime} \in \mathbb{R}^{(p+1) N} \\
\boldsymbol{E} & =\left[\bigoplus_{s=1}^{N}\left(\mathbf{I}_{p+1} \otimes U^{(s)}\right)\right] \epsilon
\end{aligned}
$$

O vetor de médias e a matriz de covariância de $Z$ são dadas por

$$
E_{\xi_{1}}(Z)=\left[\bigoplus_{s=1}^{N}\left(\mathrm{I}_{p+1} \otimes 1_{M_{s}}\right)\right] \mu
$$

$$
\operatorname{Var}_{\xi_{1}}(Z)=\stackrel{\oplus}{N=1}_{s=1}^{N}\left(\Sigma_{s} \otimes \mathbf{P}_{M_{s}}\right)
$$

\subsubsection{Incorporação da informação adicional das covariáveis}

Como conhecemos as médias das $p$ covariáveis nos $N$ estratos temos as restrições $M_{s}^{-1} 1_{M_{s}}^{\prime} \boldsymbol{X}_{s}^{(k)}=\mu_{s}^{(k) 2}, k=1, \ldots, p$. Para incorporar estas restrições, centramos os elementos de $\boldsymbol{X}_{s}^{(k)}$. Seja $\mathbf{P}_{M_{s}}=\mathbf{I}_{M_{s}}-\frac{1}{M_{s}} \mathbf{J}_{M_{s}}$, de forma que $\mathbf{P}_{M_{s}} \boldsymbol{X}^{(k)}=\boldsymbol{X}^{(k)}-$ $\mu_{s}^{(k)} 1_{M_{s}}$. Definindo

$$
\mathrm{C}=\underset{\oplus=1}{N}\left(\begin{array}{cc}
\mathrm{I}_{M I_{s}} & 0_{M_{s} \times p, M_{s}} \\
0_{p, M_{s} \times . I_{s}} & \mathrm{I}_{p} \otimes \mathrm{P}_{M_{s}}
\end{array}\right)
$$

$$
{ }^{2} \boldsymbol{X}_{s}^{(k)}=\boldsymbol{U}^{(s)} \mathbf{x}^{(k)}
$$


temos

$$
Z^{\star}=\mathrm{C} Z=\left(\begin{array}{c}
Y_{1} \\
\boldsymbol{X}_{1}^{(1)}-\mu_{1}^{(1)} 1_{M_{1}} \\
\vdots \\
\boldsymbol{X}_{1}^{(p)}-\mu_{1}^{(p)} 1_{M_{1}} \\
\vdots \\
Y_{N} \\
\boldsymbol{X}_{N}^{(1)}-\mu_{N}^{(1)} 1_{M_{N}} \\
\vdots \\
\boldsymbol{X}_{N}^{(p)}-\mu_{N}^{(p)} 1_{M_{N}}
\end{array}\right)
$$

com

$$
E_{\xi_{1}}\left(Z^{\star}\right)=\mathrm{C} E_{\xi_{1}}(Z)=\left[\underset{\oplus}{N}\left(\begin{array}{c}
1_{M_{s}} \\
0_{p M_{s}}
\end{array}\right)\right] \mu^{(y)}
$$

e

$$
\operatorname{Var}_{\xi_{1}}\left(Z^{\star}\right)=\operatorname{Var}_{\xi_{1}}(Z)
$$

Obtemos então um novo modelo dado por

$$
Z^{\star}=\mathrm{C} Z=\mathrm{CF} \mu+\mathrm{C} E=\mathrm{F}^{\star} \mu^{(y)}+E^{\star}
$$

em que

$$
\mathrm{F}^{\star}=\underset{s=1}{\oplus}\left(\begin{array}{c}
\mathbf{1}_{M_{s}} \\
0_{p, M_{s}}
\end{array}\right)
$$

\subsubsection{Amostragem estratificada e especificação de elementos amostrados e não amostrados}

Para a população de interesse, consideremos um processo de amostragem estratificado segundo o qual escolhemos uma amostra aleatória simples sem reposição de tamanho $m_{s}\left(m_{s} \leq M_{s}\right)$ em cada um dos $s$ estratos. $s:=1, \ldots, N$. Subdividamos o vetor $Z^{\star}$ em duas partes: uma parte amostrada (identificada pelo índice $S$ ) e outra não amostrada (identificada pelo índice $R$ ). Deste modo,

$$
\left(\begin{array}{c}
Z_{S}^{\star} \\
Z_{R}^{\star}
\end{array}\right)=\left(\begin{array}{l}
\mathrm{R}_{1} \\
\mathrm{R}_{2}
\end{array}\right) Z^{\star}
$$


em que

$$
\mathbf{R}_{i}=\bigoplus_{s=1}^{N}\left(\mathbf{I}_{p+1} \otimes \mathrm{I}_{i \bullet}^{(s)}\right) \in \mathbb{R}^{(p+1) M \times(p+1) M} \quad i=1,2
$$

e $\mathbf{I}_{i \bullet}^{(s)}, i=1,2$ vem da seguinte partição da matriz identidade $\mathbf{I}_{M_{s}}$ :

$$
\begin{aligned}
& \mathbf{I}_{1 \bullet}^{(s)}=\left(\mathbf{I}_{m_{\dot{v}}}, 0_{m_{s} \times\left(M_{s}-m_{s}\right)}\right) \\
& \mathbf{I}_{2 \bullet}^{(s)}=\left(\mathbf{0}_{\left(M_{s}-m_{s}\right) \times m_{s}}, \mathbf{I}_{M_{s}-m_{s}}\right) .
\end{aligned}
$$

A partir dos momentos de $Z^{\star}$, obtemos (ver a seção C.2.2 Apêndice C):

1) $E_{\xi_{1}}\left[\left(\begin{array}{c}Z_{S}^{\star} \\ Z_{R}^{\star}\end{array}\right)\right]=\left(\begin{array}{c}\mathbf{F}_{S}^{\star} \\ \mathbf{F}_{R}^{\star}\end{array}\right) \mu^{(y)}, \operatorname{com} \mathbf{F}_{S}^{\star}=\underset{s=1}{N}\left(\begin{array}{c}1_{m_{s}} \\ 0_{p m_{s}}\end{array}\right)$ e $\mathbf{F}_{R}^{\star}=\underset{s=1}{N}\left(\begin{array}{c}1_{M_{s}-m_{s}} \\ \left.0_{p\left(M_{s}-m_{s}\right)}\right)\end{array}\right)$ e

2) $\operatorname{Var}_{\xi_{1}}\left[\left(\begin{array}{c}Z_{S}^{\star} \\ Z_{R}^{\star}\end{array}\right)\right]=\left(\begin{array}{cc}\mathrm{V}_{S} & \mathrm{~V}_{S R} \\ \mathrm{~V}_{R S} & \mathrm{~V}_{R}\end{array}\right)$, com

$$
\begin{gathered}
\mathrm{V}_{S}=\bigoplus_{s=1}^{N}\left[\Sigma_{s} \otimes\left(\mathrm{I}_{m_{s}}-M_{s}^{-1} \mathbf{J}_{m_{s}}\right)\right] \\
\mathrm{V}_{S R}=\bigoplus_{s=1}^{N}\left[\Sigma_{s} \otimes\left(-M_{s}\right)^{-1} \mathbf{J}_{m_{s} \times\left(M_{s}-m_{s}\right)}\right]=\mathrm{V}_{R S}^{\prime}
\end{gathered}
$$

$\mathrm{e}$

$$
\mathrm{V}_{R}=\underset{s=1}{N}\left[\Sigma_{s} \otimes\left(\mathbf{I}_{M_{s}-m_{s}}-M_{s}^{-1} \mathbf{J}_{M_{s}-m_{s}}\right)\right]
$$

\subsubsection{Estimação da resposta total populacional e em cada estrato usando informação das covariáveis}

Queremos estimar $T^{(y)}=\left(M_{1} \mu_{1}^{(y)}, \ldots, M_{N} \mu_{N}^{(y)}\right)^{\prime}=\mathbf{L}^{\prime} Z^{\star} \in \mathbb{R}^{N}$ com $\mathrm{L}^{\prime}=\underset{s=1}{\stackrel{N}{\oplus}}\left(1_{M_{s}}^{\prime}, 0_{p, I_{s}}^{\prime}\right)$ ou, equivalentemente, $T^{(y)}=\mathrm{L}_{S}^{\prime} Z_{S}^{\star}+\mathrm{L}_{R}^{\prime} Z_{R}^{\star}$ com

$$
\begin{aligned}
& \mathrm{L}_{S}^{\prime}=\bigodot_{s=1}^{N}\left(1_{m_{s}}^{\prime}, 0_{p m_{s}}^{\prime}\right) \\
& \mathrm{L}_{R}^{\prime}=\bigodot_{s=1}^{N}\left(1_{M M_{s}-m_{s}}^{\prime} \cdot 0_{p\left(1 I_{s}-m_{s}\right)}^{\prime}\right)
\end{aligned}
$$

incorporando a informação adicional fornccida pelas covariáveis. 
Procuramos um estimador $\widehat{T}^{(y)}$ que seja uma função linear homogénea dos valores amostrados e não viesada para $T^{(y)}$, i.e.,

$$
\widehat{T}^{(y)}=\mathbf{L}_{S}^{\prime} Z_{S}^{\star}+\mathrm{W}^{\prime} Z_{S}^{\star} \quad \text { com } \quad \mathrm{W}^{\prime} \in \mathbb{R}^{N \times(p+1)\left(\sum_{s} m_{s}\right)}
$$

e

$$
E\left(\widehat{T}^{(y)}-T^{(y)}\right)=0, \forall T^{(y)}
$$

Então,

$$
0=E\left(\widehat{T}^{(y)}-T^{(y)}\right)=E\left(\mathrm{~W}^{\prime} Z_{S}^{\star}-\mathbf{L}_{R}^{\prime} Z_{R}^{\star}\right)=\left(\mathrm{W}^{\prime} \mathrm{F}_{S}^{\star}-\mathrm{L}_{R}^{\prime} \mathrm{F}_{R}^{\star}\right) \mu^{(y)}, \forall \mu^{(y)}
$$

Isto gera a restrição $\mathrm{W}^{\prime} \mathrm{F}_{S}^{\star}-\mathrm{L}_{R}^{\prime} \mathrm{F}_{R}^{\star}=0$.

Consideramos como critério de otimalidade minimizar o erro quadrático médio ou, devido à ausència de vicio, o traço da matriz de covariâncias do estimador $\widehat{T}^{(y)}$. Como

$$
\begin{aligned}
\operatorname{Var}\left(\widehat{T}^{(y)}\right) & =\left(\mathbf{L}_{S}^{\prime}+\mathbf{W}^{\prime}\right) \operatorname{Var}_{\xi_{1} \xi_{2}}\left(Z_{S}^{\star}\right)\left(\mathbf{L}_{S}+\mathbf{W}\right) \\
& =\mathbf{L}_{S}^{\prime} \mathbf{V}_{S} \mathbf{L}_{S}^{\prime}+\mathbf{L}_{S}^{\prime} \mathbf{V}_{S} \mathbf{W}+\mathbf{W}^{\prime} \mathbf{V}_{S} \mathbf{L}_{S}+\mathbf{W}^{\prime} \mathbf{V}_{S} \mathbf{W}
\end{aligned}
$$

e $\mathbf{L}_{R}^{\prime} \mathbf{V}_{S} \mathbf{L}_{R}$ não depende de $\mathbf{W}$, nosso objetivo é minimizar

$$
\phi(\mathbf{W}, \boldsymbol{\lambda})=\operatorname{tr}\left(\mathbf{W}^{\prime} \mathbf{V}_{S} \mathbf{W}-\mathbf{L}_{S}^{\prime} \mathbf{V}_{S} \mathbf{W}-\mathrm{W}^{\prime} \mathbf{V}_{S} \mathbf{L}_{S}\right)+2 \lambda^{\prime}\left(\mathbf{W}^{\prime} \mathrm{F}_{S}^{\star}-\mathbf{L}_{R}^{\prime} \mathbf{F}_{R}^{*}\right)
$$

para $\lambda \in \mathbb{R}^{N}$. As derivadas parciais de $\phi(\mathbf{W}, \boldsymbol{\lambda})$ com relação a $\mathrm{W}$ e $\boldsymbol{\lambda}$ são dadas por

$$
\begin{aligned}
\frac{\partial \phi}{\partial W} & =2 \mathrm{~V}_{S} \mathbf{W}-2 \mathrm{~V}_{S} \mathbf{L}_{S}+2 \mathrm{~F}_{S}^{\star} \lambda^{\prime} \\
\frac{\partial \phi}{\partial \boldsymbol{\lambda}} & =2\left(\mathbf{W}^{\prime} \mathbf{F}_{S}^{\star}-\mathbf{L}_{R}^{\prime} \mathbf{F}_{R}^{\star}\right)
\end{aligned}
$$

Igualando estas expressões a zero, obtemos o seguinte sistema de equações

$$
\left(\begin{array}{cc}
\mathrm{V}_{S} & \mathrm{~F}_{S}^{\star} \\
\mathrm{F}_{S}^{* \prime} & 0
\end{array}\right)\left(\begin{array}{c}
\mathrm{W} \\
\lambda^{\prime}
\end{array}\right)=\left(\begin{array}{c}
-\mathrm{V}_{S} \mathrm{~L}_{S} \\
\mathrm{~F}_{R}^{* \prime} \mathrm{L}_{R}
\end{array}\right) .
$$

Para $\mathrm{V}_{S}$ inversível. a solução é dada por

$$
\hat{\lambda}^{\prime}=-\left(\mathrm{F}_{S}^{\star \prime} \mathrm{V}_{S}^{-1} \mathrm{~F}_{S}^{\star}\right)^{-1}\left(\mathrm{~F}_{R}^{\star \prime} \mathrm{L}_{R}^{\prime}+\mathrm{F}_{S}^{\star \prime} \mathrm{L}_{S}\right)
$$


e

$$
\widehat{\mathrm{W}}=-\mathbf{L}_{S}+\mathrm{V}_{S}^{-1} \mathbf{F}_{S}^{\star}\left(\mathbf{F}_{S}^{\star \prime} \mathbf{V}_{S}^{-1} \mathbf{F}_{S}^{\star}\right)^{-1}\left(\mathbf{F}_{S}^{\star \prime} \mathbf{L}_{S}+\mathbf{F}_{R}^{\star \prime} \mathbf{L}_{R}\right)
$$

de modo que

$$
\begin{aligned}
\widehat{T}^{(y)} & =\mathrm{L}_{S}^{\prime} Z_{S}^{\star}+\widehat{\mathrm{W}}^{\prime} Z_{S}^{\star} \\
& =\left[\mathrm{V}_{S}^{-1} \mathrm{~F}_{S}^{\star}\left(\mathrm{F}_{S}^{\star \prime} \mathrm{V}_{S}^{-1} \mathrm{~F}_{S}^{\star}\right)^{-1}\left(\mathrm{~F}_{S}^{\star \prime} \mathrm{L}_{S}+\mathrm{F}_{R}^{\star \prime} \mathrm{L}_{R}\right)\right]^{\prime} Z_{S}^{\star}
\end{aligned}
$$

Calculando cada componente de $\widehat{T}^{(y)}$ (ver seção C.2.3 no Apêndice C) obtemos

$$
\widehat{T}^{(y)}=\left(\begin{array}{c}
M_{1}\left[\bar{Y}_{S 1}-\sum_{k=1}^{p} \beta_{1 ; y ; k}\left(\bar{X}_{S 1}^{(k)}-\mu_{1}^{(k)}\right)\right] \\
\vdots \\
M_{N}\left[\bar{Y}_{S N}-\sum_{k=1}^{p} \beta_{N ; y ; k}\left(\bar{X}_{S N}^{(k)}-\mu_{N}^{(k)}\right)\right]
\end{array}\right)
$$

em que

$$
\boldsymbol{\beta}_{s ; y}=\left(\beta_{s ; y, 1}, \ldots, \beta_{s ; y, p}\right)^{\prime}=\Sigma_{s ; X}^{-1} \sigma_{s ; X y}
$$

com $\sigma_{s ; X y}$ e $\Sigma_{s ; X}$ definidas em (4.13) e (4.14),

$$
\bar{Y}_{S}=\left(\bar{Y}_{S 1}, \ldots, \bar{Y}_{S N}\right)^{\prime}=\left[\underset{\oplus=1}{\oplus} \frac{1}{m_{s}} 1_{m_{S}}^{\prime}\right] Y_{S}
$$

$\mathrm{e}$

$$
\bar{X}_{S}^{(k)}=\left(\bar{X}_{S 1}^{(k)}, \ldots, \bar{X}_{S N}^{(k)}\right)^{\prime}=\left[{\underset{\Theta s}{N}}_{s=1}^{N} \frac{1}{m_{s}} 1_{m_{S}}^{\prime}\right] \boldsymbol{X}_{S}^{(k)}, \quad k=1, \ldots, p
$$

Note que o estimador obtido para a resposta total em cada estrato é do tipo regressão, corrigido por todas as covariáveis centradas.

A matriz de covariâncias de $\widehat{T}^{(y)}$ é dada por

$$
\begin{aligned}
\operatorname{Var}\left(\widehat{\boldsymbol{T}}^{(y)}\right) & =\left(\mathbf{F}_{S}^{\star \prime} \mathbf{L}_{S}+\mathbf{F}_{R}^{* \prime} \mathbf{L}_{R}\right)^{\prime}\left(\mathbf{F}_{S}^{*^{\prime}} \mathbf{V}_{S}^{-1} \mathbf{F}_{S}^{\star}\right)^{-1}\left(\mathbf{F}_{S}^{\star \prime} \mathbf{L}_{S}+\mathbf{F}_{R}^{\star \prime} \mathbf{L}_{R}\right) \\
& =\underset{s=1}{N} \frac{M_{s}\left(M_{s}-m_{s}\right)}{m_{s}} \sigma_{s: y}^{2}\left(1-\rho_{s: y}^{2}\right) \\
& =\underset{s=1}{\underset{\oplus}{N}} M_{s} \frac{1}{f_{s}}\left(1-f_{s}\right) \sigma_{s ; y}^{2}\left(1-\rho_{s: y}^{2}\right)
\end{aligned}
$$

em que $f_{s}=\frac{m_{s}}{M I_{s}}$ denota a fração de amostragem no estrato $s, s=1 \ldots \ldots$ e $\rho_{s: y}^{2}$ é definido em (C.7).

Da mesma forma, o estimador do total populacional, da resposta média em cada estrato e da resposta média populacional são dados por

$$
\widehat{T}^{(j)}=\sum_{s=1}^{N} M_{s}\left[\bar{Y}_{S s}-\sum_{k=1}^{p} \beta_{s: y: k}\left(\bar{X}_{S s}^{(k)}-\mu_{s}^{(k)}\right)\right]
$$




$$
\widehat{\mu}^{(y)}=\left(\begin{array}{c}
{\left[\bar{Y}_{S 1}-\sum_{k=1}^{p} \beta_{1 ; y ; k}\left(\bar{X}_{S 1}^{(k)}-\mu_{1}^{(k)}\right)\right]} \\
\vdots \\
{\left[\bar{Y}_{S N}-\sum_{k=1}^{p} \beta_{N ; y ; k}\left(\bar{X}_{S N}^{(k)}-\mu_{N}^{(k)}\right)\right]}
\end{array}\right)
$$

e

$$
\widehat{\mu}^{(y)}=\frac{1}{M} \sum_{s=1}^{N} M_{s}\left[\bar{Y}_{S s}-\sum_{k=1}^{p} \beta_{s ; y ; k}\left(\bar{X}_{S s}^{(k)}-\mu_{s}^{(k)}\right)\right]
$$

com

$$
\begin{gathered}
\operatorname{Var}\left(\widehat{T}^{(y)}\right)=\sum_{s=1}^{N} M_{s} \frac{1}{f_{s}}\left(1-f_{s}\right) \sigma_{s ; y}^{2}\left(1-\rho_{s ; y}^{2}\right) \\
\operatorname{Var}\left(\widehat{\boldsymbol{\mu}}^{(y)}\right)=\bigoplus_{s=1}^{N}\left(1-f_{s}\right) \frac{1}{m_{s}} \sigma_{s ; y}^{2}\left(1-\rho_{s ; y}^{2}\right)
\end{gathered}
$$

e

$$
\operatorname{Var}\left(\widehat{\mu}^{(y)}\right)=\sum_{s=1}^{N}\left(1-f_{s}\right)\left(\frac{M_{s}}{M}\right)^{2} \frac{1}{m_{s}} \sigma_{s ; y}^{2}\left(1-\rho_{s ; y}^{2}\right)
$$

respectivamente.

Note que (4.15) e (4.16) são versões generalizadas do estimador regressão separado do total ou da média em presença de várias covariáveis, sob amostragem estratificada sem reposição (e (4.17) e (4.18) são suas respectivas variâncias) que podem ser encontrados, por exemplo, nos livros de Särndal, Swensson e Wretman (1992) ou Bolfarine e Bussab (2005). 


\section{Capítulo 5}

\section{Conclusões}

\subsection{Considerações finais}

Neste trabalho centramos nosso estudo na estimação e predição ótima de diferentes quantidades populacionais com base em dados obtidos a partir de uma amostragem com dois estágios de uma população finita formada por grupos. Em uma primeira etapa, comparamos os preditores dos valores latentes correspondentes aos grupos realizados obtidos sob diferentes modelos. Para formular um modelo probabilístico que relacione a população finita com as variáveis aleatórias que permitem fazer inferências usando um enfoque preditivo consideramos os preditores propostos por Stanek e Singer (2004). Estes preditores têm a vantagem de não exigir suposições distribucionais além da existência dos momentos de primeira e segunda ordem, sendo aplicáveis tanto para variáveis contínuas quanto discretas. Comparamo-los com os preditores obtidos sob os modelos linear misto ou linear misto generalizado (para populações infinitas), consoante a variável resposta fosse contínua ou discreta e também com os preditores obtidos sob o modelo desenvolvido para populações finitas por Scott e Smith (1969) e estendido por Bolfarine e Zaks (1992). O estudo de simulação mostrou que o preditor obtido sob o modelo de permutação aleatória geralmente apresenta EQM mínimo, tanto para variáveis contínuas quanto discretas. com variancias dentro dos grupos iguais ou diferentes.

Todos os preditores envolvidos na comparação são construídos como uma combinação linear dos valores observados na amostra com preditores da resposta nas 
unidades não observadas, diferindo nas constantes de encolhimento usadas que são funções das componentes de variância populacionais. Na prática, estas componentes são desconhecidas e é de interesse tanto estimá-las quanto identificar posteriormente o modelo que fornece os melhores preditores empíricos. Neste trabalho obtivemos esses preditores empíricos sob os três modelos e comparamo-los por meio de um estudo de simulação. No caso de variáveis discretas, incluimos na comparação o preditor obtido sob o modelo linear generalizado em substituição daquele obtido sob o modelo linear misto. O estudo de simulação revelou que o preditor empírico obtido sob o modelo de permutação aleatória é o mais estável no sentido de apresentar o menor erro quadrático médio em alguns casos ou ser equivalente a ele em outros casos, em quase todas as situações consideradas. Aliás, seu erro quadrático médio nunca supera àquele do melhor preditor em mais de $50 \%$, diferentemente de seus competidores que, em algumas situações, apresentam erros quadráticos médios muito superiores àquele correspondente ao melhor preditor. Além disso, o desempenho deste preditor melhora à medida que aumentam o número, o tamanho e a fração de amostragem dos grupos. Em vista disso, podemos recomendar seu uso para uma ampla gama de condições.

Na prática a utilização de covariáveis é bastante comum. Li (2003) e Li e Stanek (2005) obtêm o estimador do total populacional desenvolvendo um modelo de permutação aleatória que, para amostragem aleatória simples, incorpora a informação de uma ou várias covariáveis e para uma amostragem estratificada, só uma covariável. Neste trabalho, para uma amostragem estratificada, estendemos os resultados de Li (2003) para situações em que o parâmetro é $q$-variado $(q \geq 1)$ e incorporamos várias covariáveis. Além disso, desenvolvemos um modelo de permutação aleatória que incorpora uma o várias covariáveis tanto para estimar parâmetros quanto para predizer variáveis aleatórias $q$-variados sob uma amostragem em dois estágios. O modelo de permutação aleatória para este tipo de amostragem tem a dificuldade adicional de considerar dois níveis de permutação (de grupos e de unidades dentro de grupos), a diferença daqueles para amostragem aleatória simples ou estratificada que usam só um nível de permutação. Os estimadores e preditores assim obtidos tèm uma correção devida ao conhecimento das covariáveis. Para sua obtenção não necessitamos de nenhum modelo adicional e a correção, que é similar àquelas obtidas em modelos de regressão, aparece como consequiência do modelado 
do processo de amostragem. Como novamente aquí esses ajustes dependem das componentes de variância e covariância populacionais, que em geral são desconhecidas, propomos estimadores para elas deixando para estudos futuros a análise da perda devida à sua incorporação.

\subsection{Futuras pesquisas}

O modelo de permutação aleatória ainda está em fase de desenvolvimento e várias extensões podem ser idealizadas.

Um dos pontos importantes é a obtenção de preditores empíricos e a avaliação teórica da perda devida ao uso dos mesmos. Nesse contexto, quando não há covariáveis presentes, seria interessante considerar outros métodos de estimação além do método de momentos. Na presença de covariáveis, ainda temos que aprofundar o estudo concernente à obtenção dos preditores empíricos, avaliando também o seu desempenho. O estudo da incorporação de covariáveis quando a única informação disponível é aquela fornecida após a amostragem é um dos pontos que precisam ser considerados, assim como a incorporação de erro na resposta.

Também é necessário disponibilizar computacionalmente as metodologias para diseminar seu uso, embora algumas delas sejam simples de implementar.

Um limitante importante no caso da amostragem em dois estágios é que os desenvolvimentos foram feitos para populações com grupos de igual tamanho e amostragem balanceada. É imprescindivel ampliar estes estudos para o caso de grupos com tamanhos desiguais e amostragem desbalanceada.

Finalmente, a ampliação do estudo a outros tipos de planejamentos e de inferência para contemplar estimação por intervalos e testes de hipótese é uma caudalosa fonte de pesquisas. 


\section{Apêndice A}

\section{Preditores e preditores empíricos Resultados adicionais}

\section{A.1 Comparação das constantes de encolhimento}

Os preditores do valor latente do $i$-ésimo grupo realizado obtidos sob o modelo linear misto $(M L M)$, sob o modelo de Scott e Smith $(M S S)$ e sob o modelo de permutação aleatória $(M P A)$ envolvem as constantes de encolhimento

$$
\begin{aligned}
k_{i}^{(L M)} & =\frac{m \sigma^{2}}{m \sigma^{2}+\sigma_{i}^{2}+\sigma_{r}^{2}}, \\
k_{i}^{(S S)} & =k_{i}^{(L M)}+f\left(1-k_{i}^{(L M)}\right)=\frac{m \sigma^{2}+f\left(\sigma_{i}^{2}+\sigma_{r}^{2}\right)}{m \sigma^{2}+\sigma_{i}^{2}+\sigma_{r}^{2}},
\end{aligned}
$$

e

$$
k^{(P A)}=\frac{m \sigma^{2}}{m \sigma^{* 2}+\sigma_{e}^{2}+\sigma_{r}^{2}}=\frac{m \sigma^{2}}{m \sigma^{2}+(1-f) \sigma_{e}^{2}+\sigma_{r}^{2}},
$$

$\operatorname{com} f=\frac{m}{M}$. Note que como $\sigma^{2}, \sigma_{i}^{2}, \sigma_{r}^{2}, \sigma_{e}^{2} \geq 0,0 \leq f \leq 1$ e $0 \leq 1-f \leq 1$ temos que
a) $m \sigma^{2} \leq m \sigma^{2}+\sigma_{i}^{2}+\sigma_{r}^{2}$ e, portanto, $0 \leq k_{i}^{(L M)} \leq 1$.
b) $f\left(\sigma_{i}^{2}+\sigma_{r}^{2}\right) \leq \sigma_{i}^{2}+\sigma_{r}^{2}$, de modo que $0 \leq h_{i}^{(S S)} \leq 1$.
c) $(1-f) \sigma_{e}^{2}+\sigma_{r}^{2} \geq 0, \log 0 \leq k^{(P .4)} \leq 1$. 
Por outro lado, como $0 \leq 1-k_{i}^{(L M)} \leq 1$ temos que $0 \leq f\left(1-k_{i}^{(L M)}\right) \leq 1$ e, por conseguinte, $k_{i}^{(S S)}=k_{i}^{(L M)}+f\left(1-k_{i}^{(L M)}\right) \leq k_{i}^{(L M)}$.

Quando as variâncias intra-grupo são idênticas (e iguais a $\sigma_{e}^{2}$ ), as constantes associadas aos preditores obtidos sob o $M L M$ ou sob o $M S S$ são, respectivamente,

$$
k^{(L M)}=\frac{m \sigma^{2}}{m \sigma^{2}+\sigma_{e}^{2}+\sigma_{r}^{2}},
$$

$\mathrm{e}$

$$
k^{(S S)}=k^{(L M)}+f\left(1-k^{(L M)}\right)=\frac{m \sigma^{2}+f\left(\sigma_{e}^{2}+\sigma_{r}^{2}\right)}{m \sigma^{2}+\sigma_{e}^{2}+\sigma_{r}^{2}},
$$

Como $0 \leq 1-f \leq 1$ temos que

$$
\begin{aligned}
& m \sigma^{2}+(1-f) \sigma_{e}^{2}+\sigma_{r}^{2} \leq m \sigma^{2}+\sigma_{e}^{2}+\sigma_{r}^{2} \\
\Leftrightarrow & \frac{m \sigma^{2}}{m \sigma^{2}+(1-f) \sigma_{e}^{2}+\sigma_{r}^{2}} \geq \frac{m \sigma^{2}}{m \sigma^{2}+\sigma_{e}^{2}+\sigma_{r}^{2}} \\
\Leftrightarrow & k^{(P A)} \geq k^{(L M)}
\end{aligned}
$$

Por outro lado,

$$
\begin{aligned}
& m f \sigma^{2} \sigma_{r}^{2}+f(1-f) \sigma_{e}^{2}\left(\sigma_{e}^{2}+\sigma_{r}^{2}\right)+f \sigma_{r}^{2}\left(\sigma_{e}^{2}+\sigma_{r}^{2}\right) \geq 0 \\
\Leftrightarrow & m f \sigma^{2} \sigma_{r}^{2}+f(1-f) \sigma_{e}^{2}\left(\sigma_{e}^{2}+\sigma_{r}^{2}\right)+f \sigma_{r}^{2}\left(\sigma_{e}^{2}+\sigma_{r}^{2}\right)+m f \sigma^{2} \sigma_{e}^{2}-m f \sigma^{2} \sigma_{e}^{2} \geq 0 \\
\Leftrightarrow & m f \sigma^{2} \sigma_{r}^{2}+f(1-f) \sigma_{e}^{2}\left(\sigma_{e}^{2}+\sigma_{r}^{2}\right)+f \sigma_{r}^{2}\left(\sigma_{e}^{2}+\sigma_{r}^{2}\right)+m f \sigma^{2} \sigma_{e}^{2}-m f \sigma^{2} \sigma_{e}^{2} \\
& m \sigma^{2} \sigma_{r}^{2}+m \sigma^{2} \sigma_{e}^{2}+m \sigma^{4} \geq m \sigma^{2} \sigma_{r}^{2}+m \sigma^{2} \sigma_{e}^{2}+m \sigma^{4} \\
\Leftrightarrow & {\left[m \sigma^{2}+f\left(\sigma_{e}^{2}+\sigma_{r}^{2}\right)\right]\left[m \sigma^{2}+(1-f)\left(\sigma_{e}^{2}+\sigma_{r}^{2}\right)\right] \geq m \sigma^{2}\left(m \sigma^{2}+\sigma_{e}^{2}+\sigma_{r}^{2}\right) } \\
\Leftrightarrow & \frac{m \sigma^{2}+f\left(\sigma_{e}^{2}+\sigma_{r}^{2}\right)}{m \sigma^{2}+\sigma_{e}^{2}+\sigma_{r}^{2}} \geq \frac{m \sigma^{2}}{m \sigma^{2}+(1-f)\left(\sigma_{e}^{2}+\sigma_{r}^{2}\right)} \\
\Leftrightarrow & k^{(S S)} \geq k^{(P A)}
\end{aligned}
$$

Deste modo. $0 \leq h^{(L . M)} \leq h^{(P .1)} \leq h^{(S S)} \leq 1$.

\section{A.2 Obtenção de preditores empíricos}

Para obter estimadores das constantes de encolhimento sob cada modelo, precisamos primeiramente estimar as componentes de variância. Vamos obter estimadores para estas componentes usando o método dos momentos, a partir das esperanças 
dos quadrados médios da análise de variância. Lembremos que estes quadrados médios podem ser expressos como formas quadráticas $Y_{S}^{* \prime} \mathrm{A} Y_{S}^{*}$, com

$$
\mathbf{A}=(n-1)^{-1}\left(\mathbf{P}_{n} \otimes \frac{\mathbf{J}_{m}}{m}\right) \quad \text { para o quadrado médio entre grupos, }
$$

$\mathrm{e}$

$$
\mathbf{A}=[n(m-1)]^{-1}\left(\mathbf{I}_{n} \otimes \mathbf{P}_{m}\right) \quad \text { para o quadrado médio dentro de grupos, }
$$

em que $\mathbf{P}_{a}=\mathbf{I}_{a}-a^{-1} \mathbf{J}_{a}$ e $a$ é um enteiro positivo. A esperança destes quadrados médios é obtida como

$$
\begin{aligned}
E\left(\boldsymbol{Y}_{S}^{* \prime} \mathbf{A} Y_{S}^{*}\right) & =\operatorname{tr}\left[\operatorname{Var}\left(\boldsymbol{Y}_{S}^{*}\right) \mathbf{A}\right]+E\left(\boldsymbol{Y}_{S}^{* \prime}\right) \mathbf{A} E\left(\boldsymbol{Y}_{S}^{*}\right) \\
& =\operatorname{tr}\left[\operatorname{Var}\left(\boldsymbol{Y}_{S}^{*}\right) \mathbf{A}\right] .
\end{aligned}
$$

O resultado $E\left(\boldsymbol{Y}_{S}^{* \prime}\right) \mathrm{A} E\left(\boldsymbol{Y}_{S}^{*}\right)=0$ é uma conseqüência de $E\left(\boldsymbol{Y}_{S}^{*}\right)=\mathbf{1}_{n m} \mu$ sob os três modelos e $\mathrm{A} 1_{n m}=0$.

Usando esta expressão, vamos obter os estimadores das constantes de encolhimento sob cada modelo em particular.

\section{A.2.1 Modelo de Permutação Aleatória}

Sob este modelo,

$$
\operatorname{Var}\left(\boldsymbol{Y}_{S}^{*}\right)=\left(\sigma_{e}^{2}+\sigma_{r}^{2}\right)\left(\mathbf{I}_{n} \otimes \mathbf{I}_{m}\right)+\sigma^{* 2}\left(\mathbf{I}_{n} \otimes \mathbf{J}_{m}\right)-\frac{\sigma^{2}}{N}\left(\mathbf{J}_{n} \otimes \mathbf{J}_{m}\right)
$$

Para o quadrado médio dentro de grupos $(Q M D), \mathbf{A}=[n(m-1)]^{-1}\left(\mathbf{I}_{n} \otimes \mathbf{P}_{m}\right)$, de modo que

$$
\operatorname{Var}\left(\boldsymbol{Y}_{S}^{*}\right) \mathbf{A}=\frac{\sigma_{e}^{2}+\sigma_{r}^{2}}{n(m-1)}\left(\mathbf{I}_{n} \otimes \mathbf{P}_{m}\right)
$$

e

$$
E(Q . M D)=\operatorname{tr}\left[\operatorname{var}\left(Y_{S}^{*}\right) \mathbf{A}\right]=\sigma_{e}^{2}+\sigma_{r}^{2} .
$$

Para o quadrado médio entre grupos $(Q M E) . \mathrm{A}=(n-1)^{-1}\left(\mathrm{P}_{n} \odot \frac{\mathbf{J}_{m}}{m}\right)$, de modo que

$$
\operatorname{Var}\left(\boldsymbol{Y}_{S}^{*}\right) \mathbf{A}=\frac{\sigma_{e}^{2}+\sigma_{r}^{2}}{n-1}\left(\mathbf{P}_{n} \otimes \frac{\mathbf{J}_{m}}{m}\right)+\frac{\sigma^{* 2}}{n-1}\left(\mathbf{P}_{n} \otimes \mathbf{J}_{m}\right)
$$


$\mathrm{e}$

$$
\begin{aligned}
E(Q M E) & =\operatorname{tr}\left[\operatorname{var}\left(\boldsymbol{Y}_{S}^{*}\right) \mathrm{A}\right] \\
& =m \sigma^{* 2}+\sigma_{e}^{2}+\sigma_{r}^{2} \\
& =m \sigma^{2}+(1-f) \sigma_{e}^{2}+\sigma_{r}^{2} .
\end{aligned}
$$

Assumindo que a variância do erro na resposta $\sigma_{r}^{2}$ é conhecida e igualando os quadrados médios observados e esperados, obtemos que $\widehat{\sigma}_{e}^{2}+\sigma_{r}^{2}=Q M D$ e $m \widehat{\sigma}^{2}+$ $(1-f) \widehat{\sigma}_{e}^{2}+\sigma_{r}^{2}=Q M E$. Assim,

$$
\begin{aligned}
m \widehat{\sigma}^{2} & =Q M E-(1-f) \widehat{\sigma}_{e}^{2}-\sigma_{r}^{2} \\
& =Q M E-(1-f)\left(Q M D-\sigma_{r}^{2}\right)-\sigma_{r}^{2} \\
& =Q M E-(1-f) Q M D-f \sigma_{r}^{2}
\end{aligned}
$$

Como $k^{(P A)}=\frac{m \sigma^{2}}{m \sigma^{2}+(1-f) \sigma_{e}^{2}+\sigma_{r}^{2}}$ satisfaz que $0 \leq k^{(P A)} \leq 1$, seu estimador também deve satisfazer esta condição. Portanto, um estimador para $k^{(P A)}$ pelo método dos momentos é

$$
\widehat{k}_{1}^{(P A)}= \begin{cases}0 & \text { se } Q M E=0 \\ \max \left(0, \frac{Q M E-(1-f) Q M D-f \sigma_{r}^{2}}{Q M E}\right) & \text { se } Q M E>0\end{cases}
$$

Se re-expressarmos $k^{(P A)}$ como

$$
k^{(P A)}=\frac{m \sigma^{2}}{m \sigma^{* 2}+\sigma_{e}^{2}+\sigma_{r}^{2}}=\frac{m \sigma^{* 2}+f \sigma_{e}^{2}}{m \sigma^{* 2}+\sigma_{e}^{2}+\sigma_{r}^{2}}=\frac{m \sigma^{* 2}+f \rho_{t}\left(\sigma_{e}^{2}+\sigma_{r}^{2}\right)}{m \sigma^{* 2}+\sigma_{e}^{2}+\sigma_{r}^{2}}
$$

em que $\rho_{t}=\sigma_{e}^{2} /\left(\sigma_{e}^{2}+\sigma_{r}^{2}\right)$, um estimador alternativo (pelo método dos momentos) para $k^{(P . A)}$ pode ser obtido assumindo que $\rho_{t}$ é conhecido. Igualando os quadrados médios observados e esperados obtemos $m \widehat{\sigma}^{* 2}+\widehat{\sigma}_{e}^{2}+\widehat{\sigma}_{r}^{2}=Q M E, \widehat{\sigma}_{\epsilon}^{2}+\widehat{\sigma}_{r}^{2}=Q M D$ e $m \widehat{\sigma}^{* 2}=Q M E-Q M D$. Assim, um estimador alternativo para $k^{(P A 1)}$ é dado por

$$
\widehat{k}_{2}^{(P . A)}= \begin{cases}0 & \text { se } Q M E=0 \\ \max \left(0, \frac{Q M E-\left(1-f \rho_{t}\right) Q M D}{Q . M E}\right) & \text { se } Q M E>0\end{cases}
$$

\section{A.2.2 Modelos de Scott e Smith e Linear Misto}

Sob o MSS e o MLM temos

$$
\operatorname{Var}\left(Y_{S}^{*}\right)=\underset{i=1}{\stackrel{\ominus}{\ominus}}\left(a_{i}^{2} \mathbf{I}_{m}+b^{2} \mathbf{J}_{m}\right)
$$


em que $a_{i}^{2}=\sigma_{i}^{2}+\sigma_{r}^{2}$ e $b^{2}=\sigma^{2}$. Para $\mathbf{A}=[n(m-1)]^{-1}\left(\mathbf{I}_{n} \otimes \mathbf{P}_{m}\right)$, temos

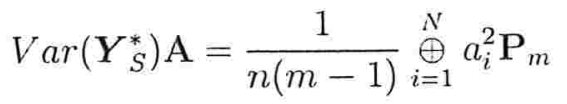

e, portanto,

$$
E(Q M D)=\operatorname{tr}\left[\operatorname{var}\left(\boldsymbol{Y}_{S}^{*}\right) \mathbf{A}\right]=\frac{1}{n} \sum_{i=1}^{n} a_{i}^{2}
$$

$$
\begin{aligned}
& \text { Para } \mathbf{A}=(n-1)^{-1}\left(\mathbf{P}_{n} \otimes \frac{\mathbf{J}_{m}}{m}\right) \text {, segue que } \\
& \operatorname{Var}\left(\boldsymbol{Y}_{S}^{*}\right) \mathbf{A}=\frac{1}{m(n-1)}\left(\left[\bigoplus_{i=1}^{N}\left(a_{i}^{2}+m b^{2}\right)\right] \mathbf{P}_{n}\right) \otimes \mathbf{J}_{m}
\end{aligned}
$$

$\mathrm{e}$

$$
\begin{aligned}
E(Q M E) & =\operatorname{tr}\left[\operatorname{var}\left(\boldsymbol{Y}_{S}^{*}\right) \mathbf{A}\right] \\
& =\frac{1}{n} \sum_{i=1}^{n} a_{i}^{2}+m b^{2}
\end{aligned}
$$

ou, equivalentemente,

$$
E(Q M E)=\frac{1}{n} \sum_{i=1}^{n}\left(a_{i}^{2}+m b^{2}\right) .
$$

Para obter os preditores empíricos, vamos distinguir os casos de variâncias intragrupos iguais ou diferentes.

\section{Variâncias intra-grupo iguais}

Quando as variâncias intra-grupo são iguais (i.e. $\sigma_{i}^{2}=\sigma_{\epsilon}^{2}$, para todo $i=$ $1, \ldots, n)$, temos $a_{i}^{2}=\sigma_{e}^{2}+\sigma_{r}^{2}$, para todo $i=1, \ldots, n$ e as esperanças dos quadrados médios se reduzem a

$$
E(Q M D)=\sigma_{e}^{2}+\sigma_{r}^{2}
$$

e

$$
E(Q M E)=m \sigma^{2}+\sigma_{e}^{2}+\sigma_{r}^{2}
$$

Igualando os quadrados médios observados e esperados obtemos que $\widehat{\sigma}_{r}^{2}+\widehat{\sigma}_{r}^{2}=$ $Q . M D, m \widehat{\sigma}^{2}+\widehat{\sigma}_{e}^{2}+\widehat{\sigma}_{r}^{2}=Q M E$ e $m \widehat{\sigma}^{2}=Q M E-Q M D$. 
Para estimar $k^{(L M)}=\frac{m \sigma^{2}}{m \sigma^{2}+\sigma_{e}^{2}+\sigma_{r}^{2}}$ (que satisfaz que $0 \leq k^{(L M)} \leq 1$ ), propomos

$$
\widehat{k}^{(L M)}= \begin{cases}0 & \text { se } Q M E=0 \\ \max \left(0, \frac{Q M E-Q M D}{Q M E}\right) & \text { se } Q M E \neq 0\end{cases}
$$

Para estimar $k^{(S S)}=k^{(L M)}+f\left(1-k^{(L M)}\right)=\frac{m \sigma^{2}+f\left(\sigma_{e}^{2}+\sigma_{r}^{2}\right)}{m \sigma^{2}+\sigma_{e}^{2}+\sigma_{r}^{2}}$ (que satisfaz que $\left.0 \leq k^{(S S)} \leq 1\right)$, temos duas alternativas:

$$
\begin{aligned}
\widehat{k}_{1}^{(S S)} & =\widehat{k}^{(L M)}+f\left(1-\widehat{k}^{(L M)}\right) \\
& = \begin{cases}f & \text { se } 0 \leq Q M E \leq Q M D \\
\max \left(0, \frac{Q M E-(1-f) Q M D}{Q M E}\right) & \text { se } Q M E>Q M D\end{cases}
\end{aligned}
$$

ou

$$
\begin{aligned}
\widehat{k}_{2}^{(S S)} & =\frac{m \widehat{\sigma}^{2}+f\left(\widehat{\sigma}_{e}^{2}+\widehat{\sigma}_{r}^{2}\right)}{m \widehat{\sigma}^{2}+\widehat{\sigma}_{e}^{2}+\widehat{\sigma}_{r}^{2}} \\
& = \begin{cases}f & \text { se } Q M E=0 \\
\max \left(0, \frac{Q M E-(1-f) Q M D}{Q M E}\right) & \text { se } Q M E>0\end{cases}
\end{aligned}
$$

Estas constantes empíricas satisfazem que $0 \leq \widehat{k}^{(L M)} \leq \widehat{k}_{2}^{(P A)} \leq \widehat{k}_{2}^{(S S)} \leq 1$, $0 \leq \widehat{k}_{1}^{(P A)} \leq \widehat{k}_{2}^{(S S)} \leq 1$ e $0 \leq \widehat{k}^{(L M)} \leq \widehat{k}_{1}^{(S S)} \leq 1$.

\section{Variâncias intra-grupo diferentes}

Considerando que

$$
Q . M D=\frac{1}{n(m-1)} \sum_{i=1}^{n} \sum_{j=1}^{m}\left(Y_{i j}^{*}-\bar{Y}_{i}^{*}\right)^{2}=\frac{1}{n} \sum_{i=1}^{n}\left[\frac{1}{m-1} \sum_{j=1}^{m}\left(Y_{i j}^{*}-\bar{Y}_{i}^{*}\right)^{2}\right]=\frac{1}{n} \sum_{i=1}^{n} S_{i}^{2}
$$

e igualando os quadrados médios observados e esperados, propomos como estimador pelo método dos momentos de $a_{i}^{2}=\sigma_{i}^{2}+\sigma_{r}^{2}$

$$
\widehat{a}_{i}^{2}=S_{i}^{2}
$$

A partir de (A.1), podemos obter como estimador de $m b^{2}(>0)$

$$
m \widehat{b}^{2}=\max (0, Q M E-Q M D)
$$


de modo que $a_{i}^{2}+m b^{2}$ pode ser estimado como

$$
\widehat{a_{i}^{2}+m b^{2}}=S_{i}^{2}+\max (0, Q M E-Q M D)=c_{i} .
$$

Da mesma forma, a partir de (A.2) e considerando que

$$
Q M E=\frac{1}{n-1} \sum_{i=1}^{n} \sum_{j=1}^{n}\left(\bar{Y}_{i}^{*}-\bar{Y}^{*}\right)^{2}=\frac{1}{n} \sum_{i=1}^{n} \frac{n m\left(\bar{Y}_{i}^{*}-\bar{Y}^{*}\right)^{2}}{n-1}=\frac{1}{n} \sum_{i=1}^{n} d_{i}^{2}
$$

podemos obter um estimador alternativo de $a_{i}^{2}+m b^{2}$, dado por

$$
\widehat{a_{i}^{2}+m} b^{2}=d_{i}^{2} .
$$

Estas expressões permitem obter dois estimadores de $k_{i}^{(L M)}=\frac{m b^{2}}{m b^{2}+a_{i}^{2}}$, dados por

$$
\widehat{k}_{i 1}^{(L M)}= \begin{cases}0 & \text { se } c_{i}=0 \\ \max \left(0, \frac{Q M E-Q M D}{c_{i}}\right) & \text { caso contrário }\end{cases}
$$

e

$$
\widehat{k}_{i 2}^{(L M)}= \begin{cases}0 & \text { se } d_{i}^{2}=0 \\ \min \left[\max \left(0, \frac{Q M E-Q M D}{d_{i}^{2}}\right), 1\right] & \text { caso contrário }\end{cases}
$$

A partir de (A.7) e (A.8) podemos obter dois estimadores de $k_{i}^{(S S)}$ como

$$
\widehat{k}_{i l}^{(S S)}=\widehat{k}_{i l}^{(L M)}+f\left(1-\widehat{k}_{i l}^{(L M)}\right) \quad \text { para. } l=1,2
$$

Um estimador alternativo de $k_{i}^{(S S)}$ pode ser obtido a partir de (A.3), (A.4) e (A.6) como

$$
\widehat{k}_{i 3}^{(S S)}=\frac{m \widehat{b}^{2}+f \widehat{a}_{i}^{2}}{m \widehat{b}^{2}+\widehat{a}_{i}^{2}}= \begin{cases}0 & \text { se } d_{i}^{2}=0 \\ \min \left[\frac{\max (0, Q M E-Q M D)+f S_{i}^{2}}{d_{i}^{2}}, 1\right] & \text { caso contrário }\end{cases}
$$

(Note que, usando (A.3), (A.4) e (A.5), chegamos à mesma expressão que $\widehat{k}_{11}^{(S S)}$. exceto quando $\left.c_{i}=0\right)$.

Para obter os preditores empíricos também precisamos substituir as componentes de variancia estimadas em

$$
\widehat{\mu}=\frac{\sum_{i=1}^{n} \bar{Y}_{i}^{*} /\left(m b^{2}+a_{i}^{2}\right)}{\sum_{i=1}^{n} 1 /\left(m b^{2}+a_{i}^{2}\right)} .
$$


A partir de (A.5) e (A.6) derivamos

$$
\widehat{\mu}_{1}=\frac{\sum_{i=1}^{n} \bar{Y}_{i}^{*} / c_{i}}{\sum_{i=1}^{n} 1 / c_{i}}
$$

$\mathrm{e}$

$$
\widehat{\mu}_{2}=\frac{\sum_{i=1}^{n} \bar{Y}_{i}^{*} / d_{i}^{2}}{\sum_{i=1}^{n} 1 / d_{i}^{2}}=\frac{\sum_{i=1}^{n} \bar{Y}_{i}^{*} /\left(\bar{Y}_{i}^{*}-\bar{Y}^{*}\right)^{2}}{\sum_{i=1}^{n} 1 /\left(\bar{Y}_{i}^{*}-\bar{Y}^{*}\right)^{2}}
$$

O primeiro é combinado com $\widehat{k}_{i 1}^{(L M)}$ e $\widehat{k}_{i 1}^{(S S)}$ e o segundo é combinado com $\widehat{k}_{i 2}^{(L M I)}$, $\widehat{k}_{i 2}^{(S S)}$ e $\widehat{k}_{i 3}^{(S S)}$ para obter os preditores empíricos. 


\section{Apêndice B}

\section{Resultados da simulação}

\section{B.1 Tabelas adicionais}

\section{Variâncias intra-grupos iguais}

Tabela B.1: Máximo acréscimo relativo percentual $(A R P)$ no EQMS para a $M G$ e os preditores obtidos sob o $M L M$, o $M S S$ e o $M P A$ com relação ao EQMS do melhor preditor, para as populações com variâncias intra-grupos iguais

\begin{tabular}{|c|c|r|r|r|}
\hline \hline \multirow{3}{*}{ População } & & \multicolumn{3}{|c|}{ Máximo ARP } \\
\cline { 3 - 5 }$N=10, M=5$ & Preditor & $F=0,2$ & $F=0,5$ & $F=0,8$ \\
\hline \hline \multirow{4}{*}{$N=10, M=20$} & $M G$ & 104,472 & 410,709 & 702,816 \\
& $M L M$ & 36,433 & 62,335 & 68,598 \\
& $M S S$ & 64,231 & 259,148 & 447,711 \\
& $M P A$ & 0,026 & 0,009 & 0,003 \\
\hline & $M G$ & 104,632 & 411,194 & 707,206 \\
& $M L M$ & 96,093 & 158,565 & 174,631 \\
& $M S S$ & 80,672 & 316,102 & 560,318 \\
& $M P A$ & 0.012 & 0,009 & 0,002 \\
\hline \multirow{4}{*}{$N=50, M=20$} & $M G$ & 907,801 & 2387,214 & 3861,398 \\
& $M L M$ & 181,850 & 194,393 & 197.749 \\
& $M S S$ & 713,619 & 1814.274 & 2966.400 \\
& $M P A$ & 0.003 & 0.001 & 0.001 \\
\hline \hline
\end{tabular}


Tabela B.2: Bom desempenho de cada preditor, para as populações com variâncias intra-grupos iguais

\begin{tabular}{|c|c|c|c|c|c|c|c|c|}
\hline \multirow[t]{2}{*}{ Populaçāo } & \multirow[t]{2}{*}{$F$} & \multirow[t]{2}{*}{ Preditor } & \multicolumn{2}{|c|}{$\begin{array}{l}\text { \% dos casos em que } \\
\text { o preditor cmpirico } \\
\text { têm EQME mínimo }\end{array}$} & \multicolumn{2}{|c|}{$\begin{array}{c}\text { \% dos casos em que } \\
\text { o preditor cmṕrico } \\
\text { é equivileute ao mellor } \\
0<A R P<15 \%\end{array}$} & \multicolumn{2}{|c|}{ Total $\%$} \\
\hline & & & $\forall p_{s}, \rho_{t}, f$ & $\begin{array}{c}\text { exiuindo } \\
\rho_{s}, \rho_{t}= \\
0,01 ; 0,99\end{array}$ & $\forall \rho_{s}, \rho_{t}, f$ & $\begin{array}{l}\text { exluindo } \\
p_{s} \cdot p_{t}= \\
0.01: 0, y 9\end{array}$ & $\forall \rho_{s}, \rho_{t}, f$ & $\begin{array}{l}\text { exluindo } \\
\rho_{x}, \rho_{t}= \\
0.01: 0.99\end{array}$ \\
\hline \multirow{3}{*}{$\begin{array}{l}N=10 \\
M=5\end{array}$} & 0,2 & $\begin{array}{c}M G \\
M L M \\
M S S \\
M P A\end{array}$ & $\begin{array}{l}36,31 \\
16,67 \\
17,86 \\
29,17\end{array}$ & $\begin{array}{l}35,56 \\
13,33 \\
24,44 \\
26,67\end{array}$ & $\begin{array}{l}16,07 \\
59,52 \\
55,36 \\
67,26\end{array}$ & $\begin{array}{l}22,22 \\
53,33 \\
57,78 \\
73,33\end{array}$ & $\begin{array}{l}52,38 \\
76.19 \\
73,22 \\
96,43\end{array}$ & $\begin{array}{r}57,78 \\
66,66 \\
82,22 \\
100,00\end{array}$ \\
\hline & 0,5 & $\begin{array}{l}\text { MG } \\
\text { MLM } \\
\text { MSS } \\
\text { MPA }\end{array}$ & $\begin{array}{l}15,48 \\
19,05 \\
38,69 \\
26,79\end{array}$ & $\begin{array}{l}14,44 \\
15,56 \\
46,67 \\
23,33\end{array}$ & $\begin{array}{l}29,76 \\
63,69 \\
19,64 \\
63,69\end{array}$ & $\begin{array}{l}33,33 \\
60,00 \\
21,11 \\
74,44\end{array}$ & $\begin{array}{l}45,24 \\
82,74 \\
58,33 \\
90,48\end{array}$ & $\begin{array}{r}7,77 \\
75.56 \\
67,78 \\
97,77\end{array}$ \\
\hline & 0,8 & $\begin{array}{l}M G \\
M L M \\
M S S \\
M P A\end{array}$ & $\begin{array}{l}0 \\
19,05 \\
41,07 * \\
41,67 *\end{array}$ & $\begin{array}{c}0 \\
14,44 \\
45,56 * \\
41,11 *\end{array}$ & $\begin{array}{l}44,05 \\
59,52 \\
16,07 \\
48,81\end{array}$ & $\begin{array}{l}45,56 \\
60,00 \\
18,89 \\
55,56\end{array}$ & $\begin{array}{l}44,05 \\
78.57 \\
57,14 \\
90.48\end{array}$ & $\begin{array}{l}45.56 \\
74.44 \\
64,45 \\
96,67\end{array}$ \\
\hline \multirow{3}{*}{$\begin{array}{l}N=10 \\
M=20\end{array}$} & 0,2 & $\begin{array}{l}M G \\
M L M \\
M S S \\
M P A\end{array}$ & $\begin{array}{l}46,94 \\
18,11 \\
16,07 \\
18,88\end{array}$ & $\begin{array}{l}49,52 \\
15,24 \\
22,38 \\
12,86\end{array}$ & $\begin{array}{l}14,54 \\
65,05 \\
72,19 \\
81.12\end{array}$ & $\begin{array}{l}20,48 \\
61,90 \\
72,86 \\
87,14\end{array}$ & $\begin{array}{r}61,48 \\
83.16 \\
88,26 \\
100,00\end{array}$ & $\begin{array}{r}70,00 \\
77.14 \\
95,24 \\
100,00\end{array}$ \\
\hline & 0.5 & $\begin{array}{c}M G \\
M L M \\
M S S \\
M P A \\
\end{array}$ & $\begin{array}{l}14,80 \\
17,09 \\
48,47 * \\
20,41 * \\
\end{array}$ & $\begin{array}{l}14,29 \\
13,33 \\
59,05 * \\
14,29 *\end{array}$ & $\begin{array}{l}40,82 \\
70,15 \\
27,81 \\
76.28 \\
\end{array}$ & $\begin{array}{l}48,10 \\
70,48 \\
27,62 \\
85,71\end{array}$ & $\begin{array}{l}55,62 \\
87,24 \\
76,28 \\
96,69 \\
\end{array}$ & $\begin{array}{r}62,39 \\
83,81 \\
86,67 \\
100,00 \\
\end{array}$ \\
\hline & 0,8 & $\begin{array}{c}\text { MG } \\
M L M \\
M S S \\
\text { MPA }\end{array}$ & $\begin{array}{c}0,51 \\
17,09 \\
51,79 * \\
31,89 * \\
\end{array}$ & $\begin{array}{c}0 \\
12,86 \\
60,00 * \\
29,05 *\end{array}$ & $\begin{array}{l}52,30 \\
69,64 \\
22,19 \\
63,52\end{array}$ & $\begin{array}{l}58,57 \\
70,95 \\
25,24 \\
70,95\end{array}$ & $\begin{array}{l}52,81 \\
86,73 \\
73,98 \\
95,41\end{array}$ & $\begin{array}{r}58,57 \\
83,31 \\
85,24 \\
100,00) \\
\end{array}$ \\
\hline \multirow{3}{*}{$\begin{array}{l}N=50 \\
M=20\end{array}$} & 0,2 & $\begin{array}{c}\text { MG } \\
\text { MLM } \\
\text { MSS } \\
\text { MPA }\end{array}$ & $\begin{array}{c}0 \\
17,09 \\
45,66 \% \\
38.01 *\end{array}$ & $\begin{array}{c}0 \\
13,33 \\
53,33 \text {. } \\
34,76 *\end{array}$ & $\begin{array}{l}49.23 \\
68.88 \\
26.53 \\
57.65\end{array}$ & $\begin{array}{l}53,81 \\
69.52 \\
30.48 \\
65,24\end{array}$ & $\begin{array}{l}49.23 \\
85.97 \\
72.19 \\
95.66\end{array}$ & $\begin{array}{r}53,81 \\
52,85 \\
83.81 \\
100,00\end{array}$ \\
\hline & (1).5 & $\begin{array}{c}M G \\
M L M \\
M I S S \\
M P A \\
\end{array}$ & $\begin{array}{c}0 \\
15,56 \\
35,97 . \\
50,26 ;\end{array}$ & $\begin{array}{c}0 \\
11,90 \\
39,05 . \\
51.43\end{array}$ & $\begin{array}{l}45,41 \\
70.41 \\
32.05 \\
45.92\end{array}$ & $\begin{array}{l}48.10 \\
70.95 \\
42.86 \\
48.57 \\
\end{array}$ & $\begin{array}{l}45,41 \\
85.97 \\
68.62 \\
96.18\end{array}$ & $\begin{array}{r}48.10 \\
82.85 \\
81,91 \\
100.00 \\
\end{array}$ \\
\hline & 0.8 & $\begin{array}{l}M G \\
M L M \\
M S S \\
\text { WHA }\end{array}$ & $\begin{array}{c}0 \\
15.52 \\
30.10 . \\
56.12,\end{array}$ & $\begin{array}{c}0 \\
12,38 \\
30,95 . \\
610,100-\end{array}$ & $\begin{array}{l}45.41 \\
69.90 \\
37.50 \\
40.05\end{array}$ & $\begin{array}{l}46.10 \\
70.45 \\
40.52 \\
40.00\end{array}$ & $\begin{array}{l}45.41 \\
85.72 \\
67.601 \\
96.17\end{array}$ & $\begin{array}{r}45.10 \\
82.86 \\
80.47 \\
106.616\end{array}$ \\
\hline
\end{tabular}


Tabela B.3: Desempenho ruim de cada preditor, para as populações com variâncias intra-grupos iguais

\begin{tabular}{|c|c|c|c|c|c|c|}
\hline \multirow[t]{2}{*}{ População } & \multirow[t]{2}{*}{$F$} & \multirow[t]{2}{*}{ Preditor } & \multicolumn{2}{|c|}{$\begin{array}{c}\% \text { dos casos em que } \\
\text { cada preditor empírico } \\
\text { têm desempenho ruim } \\
\text { com relação ao melhor } \\
\qquad A R P>50 \%\end{array}$} & \multicolumn{2}{|c|}{ Máximo $A R P(\%)$} \\
\hline & & & $\forall \rho_{s}, \rho_{t}, f$ & $\begin{array}{c}\text { exluindo } \\
\rho_{s}, \rho_{t}= \\
0,01 ; 0,99\end{array}$ & $\forall \rho_{s}, \rho_{t}, f$ & $\begin{array}{c}\text { exluindo } \\
\rho_{s}, \rho_{t}= \\
0,01 ; 0,99\end{array}$ \\
\hline \multirow{8}{*}{$\begin{array}{c}N=10 \\
M=5\end{array}$} & \multirow{2}{*}{0,2} & $M G$ & 5,95 & 0 & 67,76 & - \\
\hline & & $M L M$ & 4,17 & 4,44 & 79,55 & 79,55 \\
\hline & \multirow{3}{*}{0,5} & $M G$ & 43,45 & 36,67 & 273,50 & 215,42 \\
\hline & & $M L M$ & 5,95 & 7,78 & 192,82 & 192,82 \\
\hline & & $M S S$ & 26,19 & 15,56 & 177,11 & 149,99 \\
\hline & \multirow{3}{*}{0,8} & $M G$ & 47,02 & 41,11 & 473,70 & 371,04 \\
\hline & & $M L M$ & 6,55 & 8,89 & 245,37 & 245,37 \\
\hline & & MSS & 32,14 & 20,00 & 305,84 & 253,97 \\
\hline \multirow{8}{*}{$\begin{array}{l}N=10 \\
M=20\end{array}$} & \multirow{2}{*}{0,2} & $M G$ & 5,10 & 0 & 53,68 & - \\
\hline & & $M L M$ & 4,34 & 5,24 & 165,14 & 165.14 \\
\hline & \multirow{3}{*}{0,5} & $M G$ & 35,46 & 26,19 & 225,10 & 205,67 \\
\hline & & $M L M$ & 4,85 & 6,19 & 468,41 & 468,41 \\
\hline & & $M S S$ & 10,97 & 4,76 & 186,19 & 162,88 \\
\hline & \multirow{3}{*}{0,8} & $M G$ & 38,27 & 29,52 & 392.25 & 351,37 \\
\hline & & $M L M$ & 5,36 & 6,19 & 608,48 & 608,48 \\
\hline & & $M S S$ & 14,29 & 7,14 & 321.28 & 266.31 \\
\hline \multirow{9}{*}{$\begin{array}{l}N=50 \\
M=20\end{array}$} & \multirow{3}{*}{0,2} & $M G$ & 39,29 & 31,43 & 500.27 & 435.34 \\
\hline & & $M L M$ & 5.61 & 6.67 & 574.51 & 574.51 \\
\hline & & $M S S$ & 14,54 & 7,14 & 414.36 & 329.91 \\
\hline & \multirow{3}{*}{0.5} & $M G$ & 41.58 & 33,81 & 1284.02 & 1054.01 \\
\hline & &.$M L M$ & 6.63 & 7.14 & 718.50 & 718.50 \\
\hline & & MSS & 18.62 & 9.52 & 1046.82 & 653.50 \\
\hline & \multirow{3}{*}{0,8} & $M G$ & 42.35 & 34.76 & 2038.30 & 1560.92 \\
\hline & & $M L L M$ & 6.89 & 7.62 & 780.11 & 780.12 \\
\hline & & MSS & 20.15 & 10.95 & 1676.37 & 857.22 \\
\hline
\end{tabular}




\section{Variâncias intra-grupos diferentes}

Tabela B.4: Máximo acréscimo relativo percentual $(A R P)$ no EQMS para a $M G$ e os preditores obtidos sob o $M L M$, o MSS e o MPA com relação ao EQMS do melhor preditor, para as populações com variâncias intra-grupos diferentes

\begin{tabular}{|c|c|r|r|r|}
\hline \hline \multirow{3}{*}{ População } & \multirow{3}{*}{ Preditor } & \multicolumn{3}{|c|}{ Máximo ARP } \\
\cline { 3 - 5 }$N=10, M=5$ & $M G$ & 104,474 & 410,704 & 702,816 \\
& $M L M$ & 36,517 & 62,415 & 68,743 \\
& $M S S$ & 64,231 & 259,148 & 447,713 \\
& $M P A$ & 0,123 & 0,054 & 0,019 \\
\hline \hline \multirow{3}{*}{$N=10, M=20$} & $M G$ & 104,634 & 411,188 & 707,205 \\
& $M L M$ & 96,231 & 158,651 & 174,742 \\
& $M S S$ & 80,672 & 316,101 & 560,317 \\
& $M P A$ & 0,190 & 0,066 & 0,015 \\
\hline \multirow{3}{*}{$N=50, M=20$} & $M G$ & 907,802 & 2387,18 & 3861,40 \\
& $M L M$ & 182,014 & 194,515 & 197,824 \\
& $M S S$ & 713,620 & 1814,28 & 2966,39 \\
& $M P A$ & 0,022 & 0,008 & 0,001 \\
\hline \hline
\end{tabular}


Tabela B.5: Bom desempenho de cada preditor, para as populações com variâncias intra-grupos diferentes

\begin{tabular}{|c|c|c|c|c|c|c|c|c|}
\hline \multirow[t]{2}{*}{ Populnç̄io } & \multirow[t]{2}{*}{$F$} & \multirow[t]{2}{*}{ Preditor } & \multicolumn{2}{|c|}{$\begin{array}{l}\% \text { dos casos em que } \\
\text { o preditor empírico } \\
\text { tèm EQME mínimo }\end{array}$} & \multicolumn{2}{|c|}{$\begin{array}{c}\% \text { dos casos eli que } \\
\text { o preditor empirico } \\
\text { é equivalente ao melhor } \\
0<A R P<15 \%\end{array}$} & \multicolumn{2}{|c|}{ Totial $\%$} \\
\hline & & & $\forall p_{s}, p_{t}, f$ & $\begin{array}{c}\text { exluindo } \\
\rho_{s}, \rho_{t}= \\
0,01 ; 0,99\end{array}$ & $\forall \rho_{s}, \rho_{t}, f$ & $\begin{array}{l}\text { exluindo } \\
p_{*}, p_{t}= \\
0,(1) 1,0,99\end{array}$ & $\forall \mu_{s}, p_{t}, f$ & $\begin{array}{c}\text { exluindo } \\
\rho_{t}, \rho_{t}= \\
0,01: 0,99\end{array}$ \\
\hline \multirow{18}{*}{$\begin{array}{l}N=10 \\
M=5\end{array}$} & \multirow{6}{*}{0,2} & $M G$ & 36,31 & 19,05 & 16,07 & 22,22 & 52,38 & 41,27 \\
\hline & & MLMI & 0 & 0 & 41,67 & 32,22 & 41,67 & 32,22 \\
\hline & & MLML & 16,$6 ; 7$ & 7,14 & 59,52 & 53,33 & 76,19 & 60,47 \\
\hline & & MSS'1 & 0 & 0 & 62,50 & 72,22 & 62,50 & 72,22 \\
\hline & & MSS' & 17,86 & 13,10 & 55,36 & 57,78 & 73,22 & 70,88 \\
\hline & & MPA & 29.17 & 14,29 & $(67,26$ & 73,33 & 96,43 & 37.62 \\
\hline & \multirow{6}{*}{0,5} & $M G$ & 25,60 & 12,50 & 17,86 & 22,22 & 43,46 & 34,72 \\
\hline & & $M L M I$ & 0 & 0 & 25,00 & 17.78 & 25,00 & 17,78 \\
\hline & & MLM2 & 37,50 & 14,88 & 7,74 & 11,11 & $45,2.4$ & 25.09 \\
\hline & & MSS1 & 7.74 & 4,76 & 42,86 & 46,67 & 50,60 & 51.43 \\
\hline & & MSS2 & 17,26 & 13,69 & 18,45 & 24,44 & 35,71 & 38.13 \\
\hline & & $M P A$ & 11,90 & 7,74 & 80,36 & 83,33 & 92,26 & 91,07 \\
\hline & \multirow{6}{*}{0,8} & $M G$ & 0,60 & 0 & 40,48 & 41,11 & 41,08 & $\$ 1.11$ \\
\hline & & $M L M 1$ & 0 & 1) & 25,60 & 20,00 & $25,60)$ & 20,00 \\
\hline & & MLME & 34,52 & 13,10 & 8,93 & 11,11 & 43,45 & 24,21 \\
\hline & & MSS1 & 0 & 0 & 50,60 & 54,44 & 50,60 & 54.44 \\
\hline & & MSS2 & 16,07 & 12,50 & 14,88 & 20,00 & 30.95 & 32,50 \\
\hline & & $M P A$ & 48,81 & 27,98 & 44,05 & 47,78 & 92,86 & 75,76 \\
\hline \multirow{18}{*}{$\begin{array}{l}N=10 \\
M=20\end{array}$} & \multirow{6}{*}{0,2} & $M G$ & 46,94 & 49,52 & 14,54 & 211,48 & 61,48 & 70.100 \\
\hline & & $M L M I$ & 0 & 0 & 75,26 & 67.62 & 75,26 & 67,62 \\
\hline & & WLM2 & 18,11 & 15,24 & 65,05 & 61,90 & 83,16 & 77.14 \\
\hline & & MSSI & 0 & 0 & 83,93 & 92,86 & 83.93 & 92,86 \\
\hline & & MSS2 & 16,07 & 22,38 & 72,19 & 72,86 & 88,26 & $95,2.4$ \\
\hline & & $M P A$ & 18,88 & 12,86 & 81,12 & 87.14 & 100,00 & 100,00 \\
\hline & \multirow{6}{*}{0,5} & $M G$ & 19.64 & 20,48 & 35,20 & 40,95 & 54,84 & 61.43 \\
\hline & & $M L . W 1$ & 0,51 & 0 & 59,69 & 58,10 & 60,20 & 58,10 \\
\hline & & MLAL & 26,79 & 15,71 & 9.69 & 12,86 & 36.48 & 28,57 \\
\hline & & MSSI & 17,86 & 22,38 & 46,68 & 51,43 & 64,54 & 73,81 \\
\hline & & MSS2 & 17,86 & 25.24 & 22,96 & 24.76 & 40,82 & 50,00 \\
\hline & & MPA & 17.35 & 16.19 & 78.83 & 81.90 & 96,18 & 98.09 \\
\hline & \multirow{6}{*}{0.8} & $M G$ & 1,53 & 0) & 50,26 & 56,67 & 51.79 & 56,67 \\
\hline & & $M L M I$ & 0 & 0 & 61.99 & (iv) .95 & 61,99 & 60,95 \\
\hline & & MLML & 26,53 & 15,24 & 8.67 & 10.95 & 35,20 & 26.19 \\
\hline & & MSSI & 5.36 & 8,57 & 57,40 & 63,33 & 62,76 & 71.90 \\
\hline & & MSS2 & 15.31 & 21,43 & 20.15 & 23.81 & 35,46 & $45,2.4$ \\
\hline & & $M P A$ & 51,28 & 54,76 & 45.15 & 44.76 & 96.43 & 99,52 \\
\hline \multirow{18}{*}{$\begin{array}{l}N=30 \\
M=20\end{array}$} & \multirow{6}{*}{0.2} & $M G$ & () & 1) & 48,98 & 53,33 & 48.98 & 53.33 \\
\hline & & $M L M$ & 0 & 0 & 61,99 & 60,95 & 61,99 & 60195 \\
\hline & & MLME & 24.74 & 14.29 & 9.69 & 10.95 & 34.43 & 25.21 \\
\hline & & $M S S I$ & 0.77 & 1.43 & (61,73 & 70.009 & 62.50 & 71.13 \\
\hline & & MSS2 & 13,52 & 19.05 & 18.11 & 22.35 & 31.63 & 41,43 \\
\hline & & $M P A$ & 60.97 & 65.24 & 36.48 & 34.76 & 97.45 & $1001.06)$ \\
\hline & \multirow{6}{*}{0.5} & $M G$ & 0 & 0 & 45.41 & 18.10 & 45.41 & $4 \times .11$ \\
\hline & & MLAMI & 0 & 0 & 61.73 & 61,43 & 61.73 & 61.43 \\
\hline & & MLMLZ & 119.97 & 3,33 & 19.6 .4 & 16.67 & 30.61 & 20.00 \\
\hline & & MSS'I & 0 & 0 & 60.71 & 70.45 & 601.71 & 711.48 \\
\hline & & MSS? & 4.85 & 6.19 & 20.112 & 30.000 & 25.77 & 36,19 \\
\hline & & $M P A$ & 84.18 & $90.4 \mathrm{~s}$ & 14.5 .4 & 9,52 & $9 \times .72$ & 100.000 \\
\hline & & $M G$ & a & 0 & 45.41 & 45.10 & 15.41 & 48.10 \\
\hline & & mont & 0.26 & 0 & 61.22 & $(51.90)$ & $61,4 s$ & 61.90 \\
\hline & & MLMS & 6.12 & 0 & 21.94 & 18.57 & 28.06 & 18.57 \\
\hline & 0.8 & $\operatorname{Mss} 1$ & 11 & 0 & 54,69 & 69.05 & 59,60 & $6 \% .05$ \\
\hline & & MSS2 & 4.59 & 5.71 & 18.62 & 25.10 & 23.21 & 33.81 \\
\hline & & MPA & 49.03 & $94,20)$ & 10.20 & 5.71 & 29.23 & 100,000 \\
\hline
\end{tabular}


Tabela B.6: Desempenho ruim de cada preditor, para as populações com variâncias intra-grupos diferentes

\begin{tabular}{|c|c|c|c|c|c|c|}
\hline \multirow[t]{2}{*}{ Populaçño } & \multirow[t]{2}{*}{$F$} & \multirow[t]{2}{*}{ Preditor } & \multicolumn{2}{|c|}{$\begin{array}{l}\text { \% dos casos em que } \\
\text { cada preditor empírico } \\
\text { têm desempenho ruim } \\
\text { com relação ao melhor } \\
\text { ARP }>50 \%\end{array}$} & \multicolumn{2}{|c|}{ Máximo ARP $(\%)$} \\
\hline & & & $\forall \rho_{s}, \rho_{l}, f$ & $\begin{array}{c}\text { exluindo } \\
\rho_{s}, \rho_{t}= \\
0.01 ; 0,09\end{array}$ & $\forall \rho_{s}, \rho_{t}, f$ & $\begin{array}{c}\text { exluindo } \\
\rho_{s}, \rho_{t}= \\
0,01: 0,99\end{array}$ \\
\hline \multirow{3}{*}{$\begin{array}{l}N=10 \\
M=5\end{array}$} & 0,2 & $\begin{array}{c}M G \\
M L M 1 \\
M L M Z\end{array}$ & $\begin{array}{l}5,95 \\
5,36 \\
4,17\end{array}$ & $\begin{array}{l}0 \\
6,67 \\
4,44\end{array}$ & $\begin{array}{l}67,78 \\
84,99 \\
79,57\end{array}$ & $\begin{array}{l}- \\
84,99 \\
79,57\end{array}$ \\
\hline & 0,5 & $\begin{array}{c}M G \\
M L M 1 \\
M L M 2 \\
M S S 1 \\
M S S 2 \\
\end{array}$ & $\begin{array}{l}44,64 \\
32,14 \\
47,62 \\
33,33 \\
44,64 \\
\end{array}$ & $\begin{array}{l}37,78 \\
30,00 \\
51,11 \\
22,22 \\
30,00\end{array}$ & $\begin{array}{r}255,65 \\
210,34 \\
38221,64 \\
171,64 \\
2263,71\end{array}$ & $\begin{array}{r}229,34 \\
210,34 \\
7425,75 \\
164,16 \\
427,26\end{array}$ \\
\hline & 0,8 & $\begin{array}{c}M G \\
M L M H 1 \\
M L M 2 \\
M S S 1 \\
\text { MSS2 }\end{array}$ & $\begin{array}{l}47,62 \\
43,45 \\
49,40 \\
35,12 \\
54,77\end{array}$ & $\begin{array}{l}+2,22 \\
40,00 \\
53,33 \\
24,44 \\
35,56\end{array}$ & $\begin{array}{r}441,70 \\
258,74 \\
44041,33 \\
293,18 \\
2605,70\end{array}$ & $\begin{array}{r}384,44 \\
258,74 \\
8533,42 \\
270,58 \\
487,62\end{array}$ \\
\hline \multirow{3}{*}{$\begin{array}{l}N=10 \\
M=20\end{array}$} & 0,2 & $\begin{array}{c}M G \\
M L M 1 \\
M L M 2 \\
\end{array}$ & $\begin{array}{l}5,10 \\
4,34 \\
4,34\end{array}$ & $\begin{array}{l}0 \\
5.24 \\
5,24\end{array}$ & $\begin{array}{r}53,68 \\
168,06 \\
165,22 \\
\end{array}$ & $\begin{array}{c}- \\
168.06 \\
165.22\end{array}$ \\
\hline & 0,5 & $\begin{array}{c}\text { MG } \\
\text { MLM1 } \\
\text { MLM2 } \\
\text { MSS1 } \\
\text { MSS2 } \\
\end{array}$ & $\begin{array}{l}36,48 \\
13,01 \\
54,85 \\
20,41 \\
42,60\end{array}$ & $\begin{array}{l}27,14 \\
13,33 \\
60,48 \\
11,90 \\
35,24\end{array}$ & $\begin{array}{r}242,69 \\
474,90 \\
341712,51 \\
200,97 \\
9375,73\end{array}$ & $\begin{array}{r}225,95 \\
474,90 \\
65800,95 \\
173,62 \\
1796,91\end{array}$ \\
\hline & 0,8 & $\begin{array}{l}\text { MG } \\
\text { MLM1 } \\
\text { MLM2 } \\
\text { MSS1 } \\
\text { MSS2 } \\
\end{array}$ & $\begin{array}{l}38,27 \\
16,58 \\
56,12 \\
25,00 \\
47,45 \\
\end{array}$ & $\begin{array}{l}29,52 \\
11,76 \\
62,38 \\
15,24 \\
39,05\end{array}$ & $\begin{array}{r}409,11 \\
614,44 \\
394948,33 \\
338,68 \\
10814,77 \\
\end{array}$ & $\begin{array}{r}375,21 \\
614,44 \\
75922.96 \\
275,07 \\
2071,21\end{array}$ \\
\hline \multirow{3}{*}{$\begin{array}{l}N=50 \\
M=20\end{array}$} & 0.2 & $\begin{array}{c}\text { NG } \\
\text { MLMI } \\
\text { ML.ML } \\
\text { MSS1 } \\
\text { MSS2 }\end{array}$ & $\begin{array}{l}39,54 \\
16,58 \\
56,63 \\
25,26 \\
19,23\end{array}$ & $\begin{array}{l}31,90 \\
14,76 \\
62,86 \\
15.24 \\
40,95\end{array}$ & $\begin{array}{r}512.29 \\
578.23 \\
458531.22 \\
427.29 \\
12585.93\end{array}$ & $\begin{array}{r}463,49 \\
578.23 \\
38141,37 \\
332,88 \\
2409,73\end{array}$ \\
\hline & 0.5 & $\begin{array}{c}\text { NG } \\
\text { MLMI } \\
\text { MLWZ } \\
\text { WSS2 } \\
\text { WSSI }\end{array}$ & $\begin{array}{l}41.58 \\
17.60 \\
59,44 \\
28.06 \\
54.34\end{array}$ & $\begin{array}{l}33.31 \\
15.24 \\
66.19 \\
17.62 \\
45,24\end{array}$ & $\begin{array}{r}1284.00 \\
720,20 \\
515273.13 \\
1048.61 \\
14146,70\end{array}$ & $\begin{array}{r}1053.97 \\
720.28 \\
99038.24 \\
622.68 \\
2766.37\end{array}$ \\
\hline & 0.8 & $\begin{array}{c}\text { MG } \\
\text { WLMI } \\
\text { WLM2 } \\
\text { MSSL } \\
\text { WSS2 }\end{array}$ & $\begin{array}{l}42.35 \\
17.60 \\
60.71 \\
30.36 \\
36.63\end{array}$ & $\begin{array}{l}34.76 \\
15.71 \\
68,10 \\
15.57 \\
46.67\end{array}$ & $\begin{array}{r}20336,91 \\
780.81 \\
530158,37 \\
16,77.70 \\
14627.60 \\
\end{array}$ & $\begin{array}{r}1560.96 \\
780.81 \\
1020386.60 \\
811.21 \\
2798.93\end{array}$ \\
\hline
\end{tabular}




\section{B.2 Figuras adicionais}

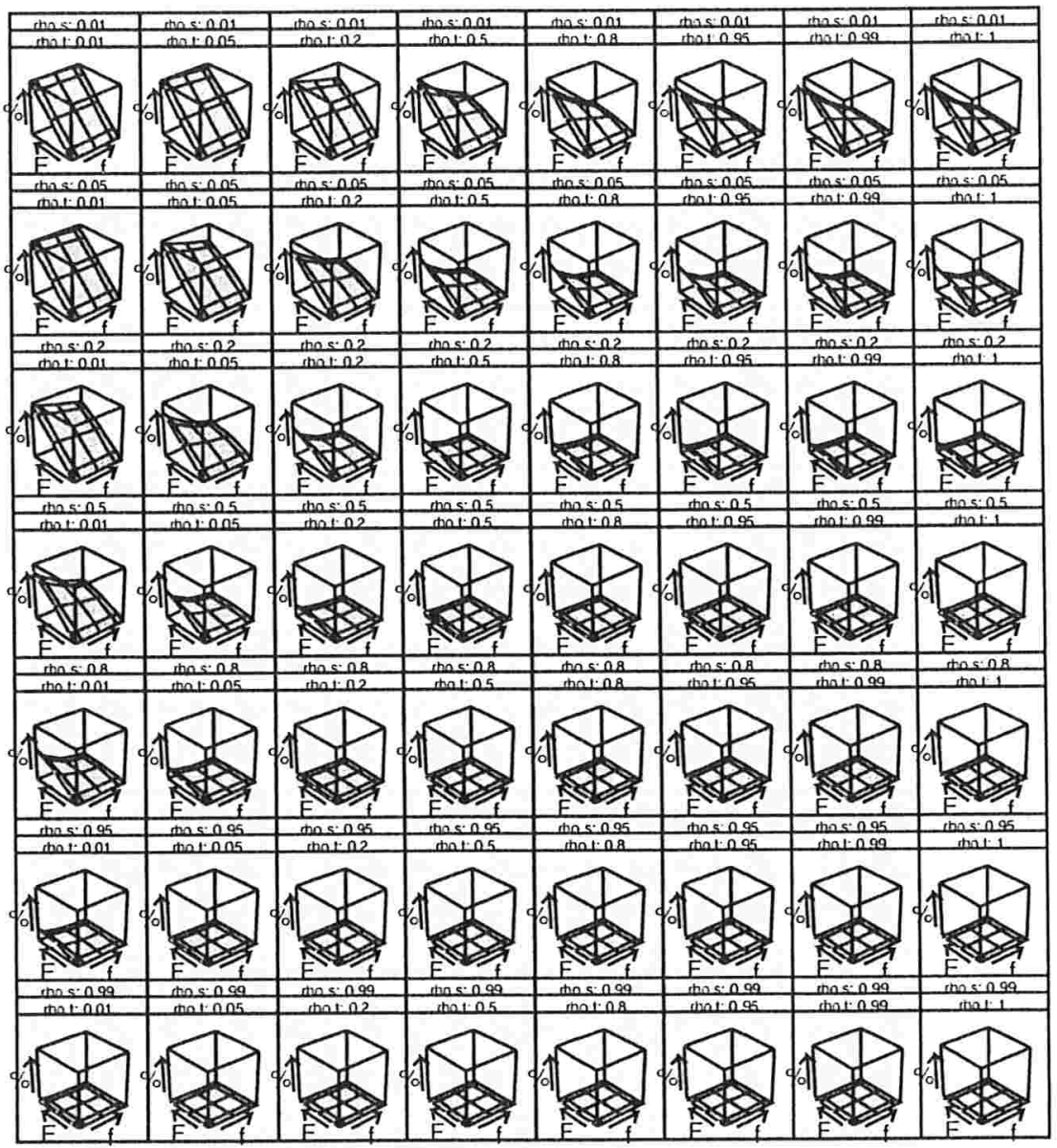

Figura B.1: Acréscimo relativo percentual no EQMS da $M G$ com relação ao EQMSS do melhor preditor para as populações com $N=10, M=20$ e variâncias intra-grupos iguais e conhecidas. O eixo denotado por $\mathrm{f}$ toma valores 0,$1 ; 0,2 ; 0,4 ; 0,5 ; 0,6 ; 0.8$ e 0,9 . O eixo denotado por $\mathrm{F}$ toma valores 0,$2 ; 0,5$ e 0,8 . O máximo valor para o eixo denotado por $\%$ é 710 . 


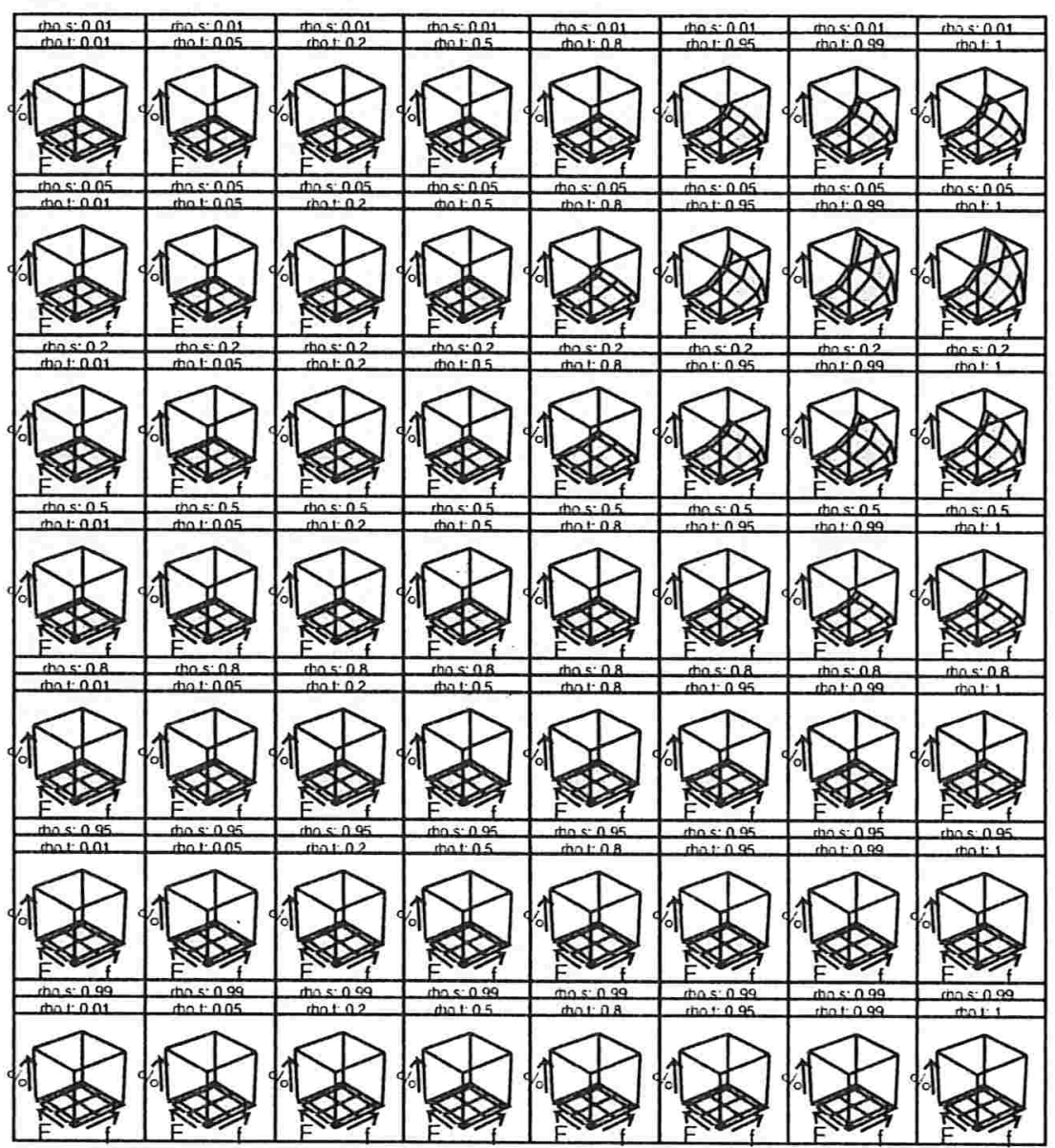

Figura B.2: Acréscimo relativo percentual no EQMS do preditor do $M L M$ com relação ao EQMS do melhor preditor para as populações $\operatorname{com} N=10, M=20$ e variancias intragrupos iguais e conhecidas. O eixo denotado por $\mathrm{f}$ toma valores $0,1: 0.2 ; 0,4 ; 0,5 ; 0.6 ; 0.8$ e 0.9. O eixo denotado por $F$ toma valores 0,$2 ; 0,5$ e 0.8 . O máximo valor para o eixo denotado por \% é 175 . 


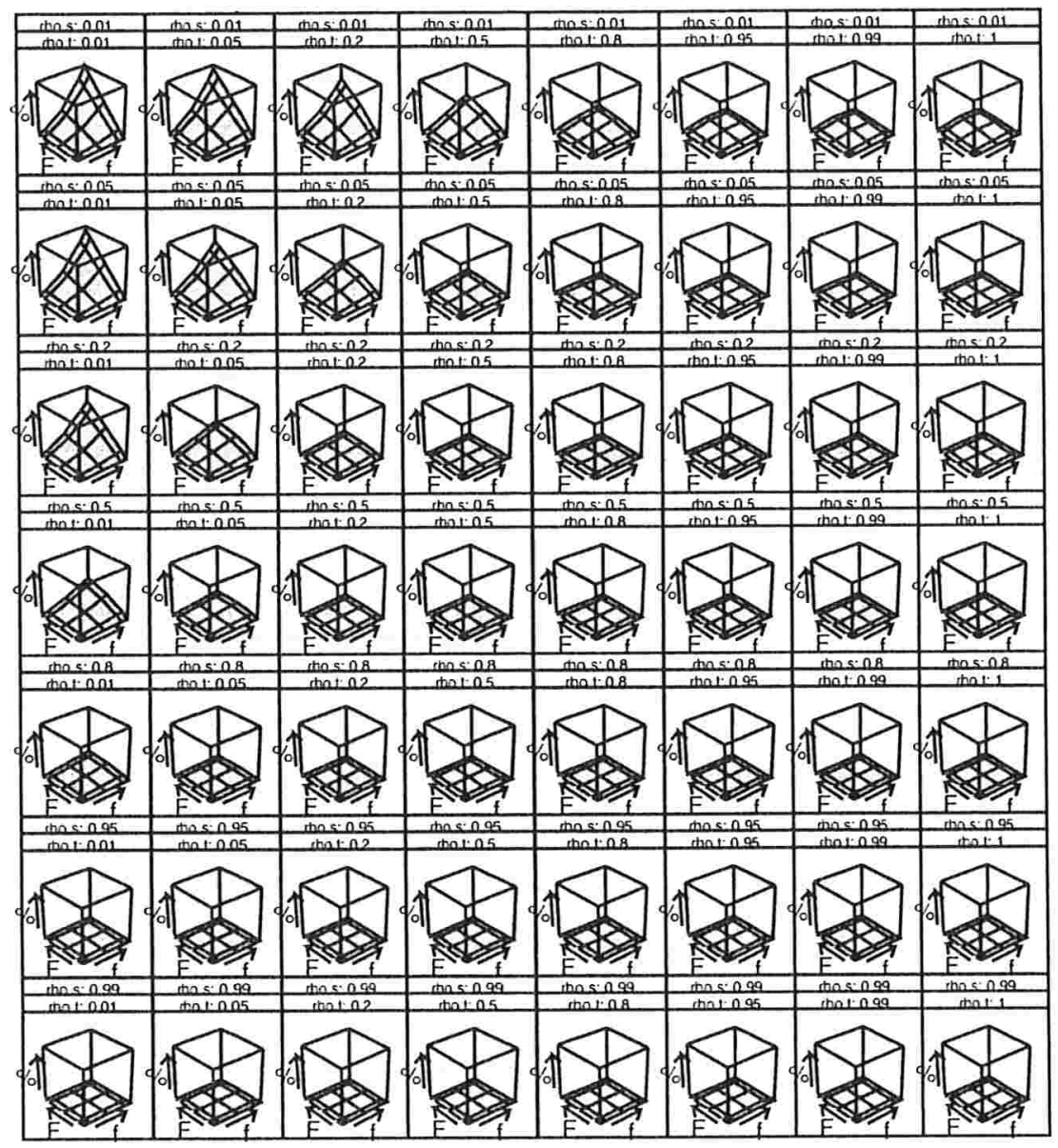

Figura B.3: Acréscimo relativo percentual no EQMS do preditor do MSS com relação ao EQMS do melhor preditor para as populaçōes com $N=10, M=20$ e variâncias intragrupos iguais e conhecidas. $O$ eixo denotado por $\mathrm{f}$ toma valores 0,$1 ; 0,2 ; 0,4 ; 0,5 ; 0,6: 0.8$ e 0,9 . O eixo denotado por $\mathrm{F}$ toma valores 0,$2 ; 0,5$ e 0,8 . O máximo valor para o eixo denotado por \% é 560 . 


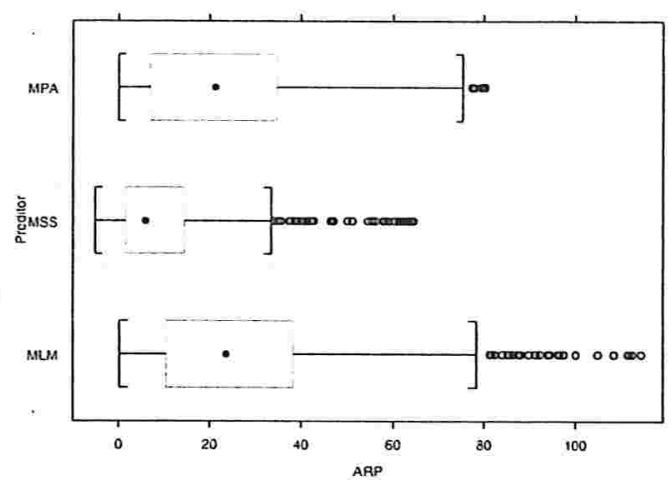

(a) Populações com $N=10, M=5$

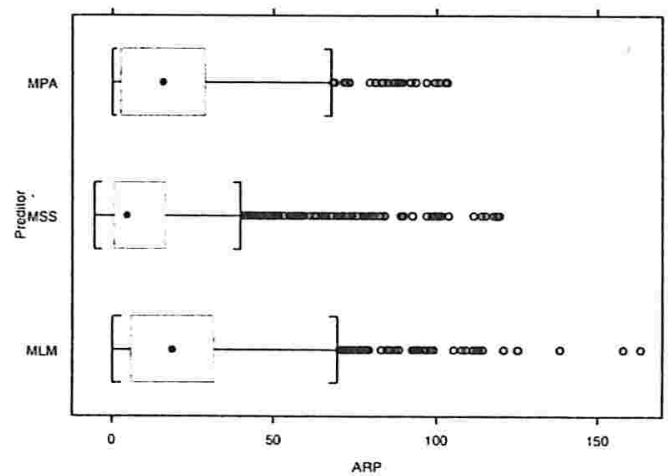

(b) Populações com $N=10, M=20$

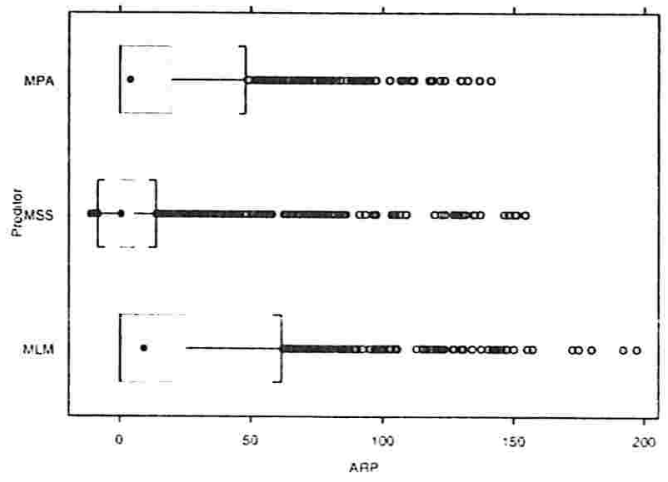

(c) Populaçós com $. V=50, M=20$

Figura B.4: Acréscimo relativo percentual no EQ.ME dos preditores empíricos com relação ao EQMS dos preditores obtidos sob o MLM, o MSS ou o MPA, para as populações com variâncias intra-grupos iguais 


\section{Apêndice C}

\section{Detalhes sobre a obtenção de estimadores e preditores ótimos em presença de covariáveis}

\section{C.1 Amostragem em dois estágios}

C.1.1 Momentos de $Y$ e $X^{(k)}$

Por simplicidade só vamos desenvolver $E_{\xi_{1} \xi_{2}}\left(\boldsymbol{X}^{(k)}\right), \quad \operatorname{Var}_{\xi_{1} \xi_{2}}\left(\boldsymbol{X}^{(k)}\right)$ e $\operatorname{Cov}_{\xi_{1} \xi_{2}}\left(\boldsymbol{X}^{(k)}, \boldsymbol{X}^{(l)}\right)$, para $k, l=1, \ldots, p ; k \neq l$. Os termos $E_{\xi_{1} \xi_{2}}(\boldsymbol{Y}), \operatorname{Var}_{\xi_{1} \xi_{2}}(\boldsymbol{Y}) \mathrm{e}$ $\operatorname{Cov}_{\xi_{1} \xi_{2}}\left(\boldsymbol{Y}, \boldsymbol{X}^{(k)}\right)$ são obtidos em forma análoga. Aqui o subíndice $\xi_{1}$ denota esperança com relação à permutação dos grupos e o subíndice $\xi_{2}$ denota esperança com relação à permutação de unidades em um grupo.

Para $k=1, \ldots, p$, temos

$$
E_{\xi_{1} \xi_{2}}\left(X^{(k)}\right)=\mu^{(k)}\left(1_{N} \otimes 1_{M}\right)
$$

e

$$
\operatorname{Cov}_{\xi_{1} \xi_{2}}\left(\boldsymbol{X}^{(k)}, \boldsymbol{X}^{(l)}\right)=\sigma_{\mu ; k, l}\left(\mathbf{P}_{N} \otimes \mathbf{J}_{M}\right)+\sigma_{e: k, l}\left(\mathbf{I}_{N} \otimes \mathbf{P}_{M}\right)
$$


com

$$
\begin{aligned}
\sigma_{\mu ; k, l} & =\frac{1}{N-1} \mu^{(k)^{\prime}} \mathrm{P}_{N} \boldsymbol{\mu}^{(l)} \\
\sigma_{e ; k, l} & =\frac{1}{N} \sum_{s=1}^{N} \sigma_{s ; k, l} \\
\sigma_{s ; k, l} & =\frac{1}{M-1} \mathrm{x}_{s}^{(k)^{\prime}} \mathbf{P}_{M} \mathrm{x}_{s}^{(l)}, \text { para } s=1, \ldots, N \\
\mu^{(k)} & =\left(\mu_{1}^{(k)}, \ldots, \mu_{N}^{(k)}\right)^{\prime} \in \mathbb{R}^{N}
\end{aligned}
$$

em que $\mu^{(k)}$ é o vetor das médias da variável $k$ para os $N$ grupos, $\sigma_{\mu ; k, l}$ é a covariância entre as médias dos grupos para as variáveis $k$ e $l$ e $\sigma_{s ; k, l}$ é a covariancia entre as variáveis $k$ e $l$ dentro do grupo $s$. Denotemos também por $\mu^{(k)} \in \mathbb{R}$ a média geral da variável $k$. Em particular, para $k=l$, temos

$$
\operatorname{Var}_{\xi_{1} \xi_{2}}\left(\boldsymbol{X}^{(k)}\right)=\sigma_{\mu ; k}^{2}\left(\mathbf{P}_{N} \otimes \mathbf{J}_{M}\right)+\sigma_{e ; k}^{2}\left(\mathbf{I}_{N} \otimes \mathbf{P}_{M}\right)
$$

$\operatorname{com} \sigma_{\mu ; k}^{2}=\sigma_{\mu ; k, k}$ e $\sigma_{e ; k}^{2}=\sigma_{e ; k, k}$

1) Obtenção de $E_{\xi_{1} \xi_{2}}\left(X^{(k)}\right)$

$$
\begin{aligned}
& E_{\xi_{1} \xi_{2}}\left(\boldsymbol{X}^{(k)}\right)=E_{\xi_{1} \xi_{2}}\left[\left(\boldsymbol{U} \otimes \mathrm{I}_{M}\right)\left(\underset{s=1}{\oplus} \boldsymbol{U}^{(s)}\right) \mathrm{x}^{(k)}\right] \\
& =E_{\xi_{1}}\left\{\left(\boldsymbol{U} \otimes \mathbf{I}_{M}\right) E_{\xi_{2} \mid \xi_{1}}\left[\left(\bigoplus_{s=1}^{N} U^{(s)}\right) \mathbf{x}^{(k)}\right]\right\} \\
& =E_{\xi_{1}}\left[\left(\boldsymbol{U} \otimes \mathbf{I}_{M}\right) M^{-1}\left(\mathbf{I}_{N} \otimes \mathbf{J}_{M}\right) \mathbf{x}^{(k)}\right]
\end{aligned}
$$

pois como $E_{\xi_{2} \mid \xi_{1}}\left(U^{(s)}\right)=M^{-1} \mathbf{J}_{M}$, temos $\bigoplus_{s=1}^{N} E_{\xi_{2} \mid \xi_{1}}\left(U^{(s)}\right)=\bigoplus_{s=1}^{N} M^{-1} \mathbf{J}_{M}=M^{-1}\left(\mathbf{I}_{N} \otimes\right.$ $\left.\mathrm{J}_{M}\right)$. Logo,

$$
\begin{aligned}
E_{\xi_{1} \xi_{2}}\left(X^{(k)}\right) & =\left(E_{\xi_{1}}(U) \otimes \mathbf{I}_{M}\right) M^{-1}\left(\mathbf{I}_{N} \otimes \mathbf{J}_{M}\right) \mathbf{x}^{(k)} \\
& =N^{-1} M^{-1}\left(\mathbf{J}_{N} \otimes \mathbf{I}_{M}\right)\left(\mathbf{I}_{N} \otimes \mathbf{J}_{M}\right) \mathbf{x}^{(k)} \\
& =N^{-1} M^{-1}\left(\mathbf{J}_{N} \otimes \mathbf{J}_{M}\right) \mathbf{x}^{(k)} \\
& =N^{-1} M^{-1}\left(\sum_{s=1}^{N} \sum_{t=1}^{M} x_{s t}^{(k)}\right)\left(1_{N} \otimes 1_{M}\right) \\
& =\mu^{(k)}\left(1_{N} \otimes 1_{M}\right)
\end{aligned}
$$

2) Obtenção da $\operatorname{Cov}_{\xi_{1} \xi_{2}}\left(\boldsymbol{X}^{(k)}, \boldsymbol{X}^{(l)}\right)$

$\operatorname{Cov}_{\xi_{1} \xi_{2}}\left(\boldsymbol{X}^{(k)}, \boldsymbol{X}^{(l)}\right)=\operatorname{Cov}_{\xi_{1}}\left[E_{\xi_{2} \mid \xi_{1}}\left(\boldsymbol{X}^{(k)}\right) \cdot E_{\xi_{2} \mid \xi_{1}}\left(\boldsymbol{X}^{(l)}\right)\right]+E_{\xi_{1}}\left[\operatorname{Cov}_{\xi_{2} \mid \xi_{1}}\left(\boldsymbol{X}^{(k)}, \boldsymbol{X}^{(l)}\right)\right]$ 
Inicialmente observemos que

$$
\begin{aligned}
\operatorname{Cov}_{\xi_{1}}\left[E_{\xi_{2} \mid \xi_{1}}\left(X^{(k)}\right), E_{\xi_{2} \mid \xi_{1}}\left(X^{(l)}\right)\right] & = \\
& =\operatorname{Cov}_{\xi_{1}}\left[M^{-1}\left(U \otimes \mathbf{I}_{M}\right)\left(\mathbf{I}_{N} \otimes \mathbf{J}_{M}\right) \mathbf{x}^{(k)}, M^{-1}\left(U \otimes \mathbf{I}_{M}\right)\left(\mathbf{I}_{N} \otimes \mathbf{J}_{M}\right) \mathbf{x}^{(l)}\right] .
\end{aligned}
$$

Mas como

$$
\begin{gathered}
\left(\mathbf{I}_{N} \otimes \mathbf{J}_{M}\right) \mathbf{x}^{(k)}=\left(\begin{array}{cccc}
\mathbf{J}_{M} & 0 & \cdots & 0 \\
0 & \mathbf{J}_{M} & \cdots & 0 \\
\vdots & \vdots & \ddots & \vdots \\
0 & 0 & \cdots & \mathbf{J}_{M}
\end{array}\right)\left(\begin{array}{c}
\mathbf{x}_{1}^{(k)} \\
\mathbf{x}_{2}^{(k)} \\
\vdots \\
\mathbf{x}_{N}^{(k)}
\end{array}\right)=\left(\begin{array}{c}
\left(\sum_{t=1}^{M} x_{1 t}^{(k)}\right) 1_{M} \\
\left(\sum_{t=1}^{M} x_{2 t}^{(k)}\right) 1_{M} \\
\vdots \\
\left(\sum_{t=1}^{M} x_{N t}^{(k)}\right) 1_{M}
\end{array}\right) \\
\Rightarrow \quad M^{-1}\left(\mathbf{I}_{N} \otimes \mathbf{J}_{M}\right) \mathbf{x}^{(k)}=\left(\begin{array}{c}
\mu_{1}^{(k)} 1_{M} \\
\vdots \\
\mu_{N}^{(k)} 1_{M}
\end{array}\right)=\mu^{(k)} \otimes 1_{M}
\end{gathered}
$$

e obtemos

$$
\begin{aligned}
\operatorname{Cov}_{\xi_{1}} & {\left[E_{\xi_{2} \mid \xi_{1}}\left(\boldsymbol{X}^{(k)}\right), E_{\xi_{2} \mid \xi_{1}}\left(\boldsymbol{X}^{(l)}\right)\right]=} \\
& =\operatorname{Cov}_{\xi_{1}}\left[\left(\boldsymbol{U} \otimes \mathbf{I}_{M}\right)\left(\boldsymbol{\mu}^{(k)} \otimes \mathbf{1}_{M}\right),\left(\boldsymbol{U} \otimes \mathbf{I}_{M}\right)\left(\boldsymbol{\mu}^{(l)} \otimes 1_{M}\right)\right] \\
& =\operatorname{Cov}_{\xi_{1}}\left[\boldsymbol{U} \boldsymbol{\mu}^{(k)} \otimes 1_{M}, U \boldsymbol{\mu}^{(l)} \otimes 1_{M}\right] \\
& =E_{\xi_{1}}\left[\left(\boldsymbol{U} \boldsymbol{\mu}^{(k)} \otimes \mathbf{1}_{M}\right)\left(\boldsymbol{\mu}^{(l)^{\prime}} \boldsymbol{U}^{\prime} \otimes \mathbf{1}_{M}^{\prime}\right)\right]-E_{\xi_{1}}\left(\boldsymbol{U} \boldsymbol{\mu}^{(k)} \otimes 1_{M}\right) E_{\xi_{1}}^{\prime}\left(\boldsymbol{U} \boldsymbol{\mu}^{(l)} \otimes \mathbf{1}_{M}\right) \\
& =E_{\xi_{1}}\left[\boldsymbol{U} \boldsymbol{\mu}^{(k)} \boldsymbol{\mu}^{(l)^{\prime}} \boldsymbol{U}^{\prime} \otimes \mathbf{J}_{M}\right]-N^{-2}\left(\mathbf{J}_{N} \boldsymbol{\mu}^{(k)} \otimes 1_{M}\right)\left(\mathbf{J}_{N} \boldsymbol{\mu}^{(k)} \otimes \mathbf{1}_{M}\right)^{\prime} \\
& =E_{\xi_{1}}\left[\operatorname{vec}\left(\boldsymbol{U} \boldsymbol{\mu}^{(k)}\right) \operatorname{vec}\left(\boldsymbol{U} \boldsymbol{\mu}^{(l)}\right)\right] \otimes \mathbf{J}_{M}-\mu^{(k)} \mu^{(l)}\left(\mathbf{1}_{N} \otimes 1_{M}\right)\left(\mathbf{1}_{N}^{\prime} \otimes \mathbf{1}_{M}^{\prime}\right) \\
& =E_{\xi_{1}}\left\{\left(\boldsymbol{\mu}^{(k)^{\prime}} \otimes \mathbf{I}_{N}\right) \operatorname{vec}(\boldsymbol{U})\left[\left(\boldsymbol{\mu}^{(l)^{\prime}} \otimes \mathbf{I}_{N}\right) \operatorname{vec}(\boldsymbol{U})\right]^{\prime}\right\} \otimes \mathbf{J}_{M}-\mu^{(k)} \mu^{(l)}\left(\mathbf{J}_{N} \otimes \mathbf{J}_{M}\right) \\
& =E_{\xi_{1}}\left[\left(\boldsymbol{\mu}^{(k)^{\prime}} \otimes \mathbf{I}_{N}\right) \operatorname{vec}(\boldsymbol{U}) \operatorname{vec}^{\prime}(\boldsymbol{U})\left(\boldsymbol{\mu}^{(l)} \otimes \mathbf{I}_{N}\right)\right] \otimes \mathbf{J}_{M}-\mu^{(k)} \mu^{(l)}\left(\mathbf{J}_{N} \otimes \mathbf{J}_{M}\right) \\
& =\left\{\left(\boldsymbol{\mu}^{(k)^{\prime}} \otimes \mathbf{I}_{M}\right) E_{\xi_{1}}\left[\operatorname{vec}(U) \operatorname{vec}^{\prime}(\boldsymbol{U})\right]\left(\boldsymbol{\mu}^{(l)} \otimes \mathbf{I}_{M}\right)\right\} \otimes \mathbf{J}_{M}-\mu^{(k)} \mu^{(l)}\left(\mathbf{J}_{N} \otimes \mathbf{J}_{M}\right)
\end{aligned}
$$

Por outro lado,

$$
\begin{aligned}
E_{\xi_{1}}\left[\operatorname{vec}(U) \operatorname{vec}^{\prime}(U)\right] & =\mathcal{D}_{\xi_{1}}[\operatorname{vec}(U)]+E_{\xi_{1}}[\operatorname{vec}(U)] E_{\xi_{1}}^{\prime}[\operatorname{vec}(U)] \\
& =\frac{1}{N-1} \mathbf{P}_{N} \otimes \mathbf{P}_{N}+\frac{1}{N^{2}}\left(\mathbf{1}_{N} \otimes 1_{N}\right)\left(\mathbf{1}_{N}^{\prime} \otimes \mathbf{1}_{N}^{\prime}\right) \\
& =\frac{1}{N-1} \mathbf{P}_{N} \otimes \mathbf{P}_{N}+\frac{1}{N^{2}}\left(\mathbf{J}_{N} \otimes \mathbf{J}_{N}\right)
\end{aligned}
$$


o que implica que

$$
\begin{aligned}
\operatorname{Cov}_{\xi_{1}} & {\left[E_{\xi_{2} \mid \xi_{1}}\left(\boldsymbol{X}^{(k)}\right), E_{\xi_{2} \mid \xi_{1}}\left(\boldsymbol{X}^{(l)}\right)\right]=} \\
& =\frac{1}{N-1}\left[\left(\boldsymbol{\mu}^{(k)^{\prime}} \otimes \mathbf{I}_{N}\right)\left(\mathbf{P}_{N} \otimes \mathbf{P}_{N}\right)\left(\boldsymbol{\mu}^{(l)} \otimes \mathbf{I}_{N}\right)\right] \otimes \mathbf{J}_{M} \\
& +\frac{1}{N^{2}}\left[\left(\boldsymbol{\mu}^{(k)^{\prime}} \otimes \mathbf{I}_{N}\right)\left(\mathbf{J}_{N} \otimes \mathbf{J}_{N}\right)\left(\boldsymbol{\mu}^{(l)} \otimes \mathbf{I}_{N}\right)\right] \otimes \mathbf{J}_{M}-\mu^{(k)} \mu^{(l)}\left(\mathbf{J}_{N} \otimes \mathbf{J}_{M}\right) \\
& =\frac{1}{N-1} \mu^{(k)^{\prime}} \mathbf{P}_{N} \boldsymbol{\mu}^{(l)} \otimes \mathbf{P}_{N} \otimes \mathbf{J}_{M}+\frac{1}{N^{2}} \boldsymbol{\mu}^{(k)^{\prime}} \mathbf{J}_{N} \boldsymbol{\mu}^{(l)} \otimes \mathbf{J}_{N} \otimes \mathbf{J}_{M} \\
& -\mu^{(k)} \mu^{(l)}\left(\mathbf{J}_{N} \otimes \mathbf{J}_{M}\right) .
\end{aligned}
$$

Agora seja $\sigma_{\mu ; k, l}=\frac{1}{N-1} \boldsymbol{\mu}^{(k)^{\prime}} \mathbf{P}_{N} \boldsymbol{\mu}^{(l)} \in \mathbb{R}$ e notemos que

$$
\boldsymbol{\mu}^{(k)^{\prime}} \mathbf{J}_{N} \boldsymbol{\mu}^{(l)}=\boldsymbol{\mu}^{(k)^{\prime}} \mathbf{1}_{N} \mathbf{1}_{N}^{\prime} \boldsymbol{\mu}^{(l)}=\left(\sum_{s=1}^{N} \mu_{s}^{(k)}\right)\left(\sum_{s=1}^{N} \mu_{s}^{(l)}\right)=N^{2} \mu^{(k)} \mu^{(l)}
$$

Logo

$$
\operatorname{Cov}_{\xi_{1}}\left[E_{\xi_{2} \mid \xi_{1}}\left(\boldsymbol{X}^{(k)}\right), E_{\xi_{2} \mid \xi_{1}}\left(\boldsymbol{X}^{(l)}\right)\right]=\sigma_{\mu ; k, l}\left(\mathbf{P}_{N} \otimes \mathbf{J}_{M}\right)
$$

Para obter $E_{\xi_{1}}\left[\operatorname{Cov}_{\xi_{2} \mid \xi_{1}}\left(\boldsymbol{X}^{(k)}, \boldsymbol{X}^{(l)}\right)\right]$, calculemos primeiramente $\operatorname{Cov}_{\xi_{2} \mid \xi_{1}}\left(\boldsymbol{X}^{(k)}, \boldsymbol{X}^{(l)}\right)$.

$$
\begin{aligned}
& \operatorname{Cov}_{\xi_{2} \mid \xi_{1}}\left(\boldsymbol{X}^{(k)}, \boldsymbol{X}^{(l)}\right)=\operatorname{Cov}_{\xi_{2} \mid \xi_{1}}\left[\left(\boldsymbol{U} \otimes \mathbf{I}_{M}\right)\left(\underset{s=1}{\stackrel{N}{\oplus}} \boldsymbol{U}^{(s)}\right) \mathbf{x}^{(k)},\left(\boldsymbol{U} \otimes \mathbf{I}_{M}\right)\left(\underset{s=1}{\oplus} \boldsymbol{U}^{(s)}\right) \mathbf{x}^{(l)}\right] \\
& =\left(\boldsymbol{U} \otimes \mathbf{I}_{M}\right) \operatorname{Cov}_{\xi_{2} \mid \xi_{1}}\left[\left(\bigoplus_{s=1}^{N} \boldsymbol{U}^{(s)}\right) \mathbf{x}^{(k)},\left(\underset{s=1}{\oplus} \boldsymbol{U}^{(s)}\right) \mathbf{x}^{(l)}\right]\left(\boldsymbol{U}^{\prime} \otimes \mathbf{I}_{M}\right)
\end{aligned}
$$

Observando que

$$
\left(\underset{s=1}{\oplus} U^{(s)}\right) \mathbf{x}^{(k)}=\left(\begin{array}{c}
N \\
\oplus=1
\end{array} U^{(s)}\right)\left(\begin{array}{c}
\mathbf{x}_{1}^{(k)} \\
\vdots \\
\mathbf{x}_{N}^{(k)}
\end{array}\right)=\left(\begin{array}{c}
U^{(1)} \mathbf{x}_{1}^{(k)} \\
\vdots \\
U^{(N)} \mathbf{x}_{N}^{(k)}
\end{array}\right),
$$

podemos concluir que

$$
\begin{aligned}
\operatorname{Cov}_{\xi_{2} \mid \xi_{1}}[ & {\left.\left[\bigoplus_{s=1}^{N} U^{(s)}\right) \mathbf{x}^{(k)},\left(\underset{s=1}{N} U^{(s)}\right) \mathbf{x}^{(l)}\right]=} \\
= & \left(\begin{array}{ccc}
\operatorname{Cov}_{\xi_{2} \mid \xi_{1}}\left(U^{(1)} \mathbf{x}_{1}^{(k)}, U^{(1)} \mathbf{x}_{1}^{(k)}\right) & \cdots & \operatorname{Cov}_{\xi_{2} \mid \xi_{1}}\left(U^{(1)} \mathbf{x}_{1}^{(k)} \cdot U^{(N)} \mathbf{x}_{N}^{(k)}\right) \\
\vdots & \ddots & \vdots \\
\operatorname{Cov}_{\xi_{2} \mid \xi_{1}}\left(U^{(N)} \mathbf{x}_{N}^{(k)}, U^{(1)} \mathbf{x}_{1}^{(k)}\right) & \cdots & \operatorname{Cov}_{\xi_{2} \mid \xi_{1}}\left(U^{(N)} \mathbf{x}_{N}^{(k)} \cdot U^{(N)} \mathbf{x}_{N}^{(k)}\right)
\end{array}\right)
\end{aligned}
$$


Para $s=1, \ldots, N$, notemos que

$$
\begin{aligned}
\operatorname{Cov}_{\xi_{2} \mid \xi_{1}}\left(U^{(s)} \mathbf{x}_{s}^{(k)}, U^{(s)} \mathbf{x}_{s}^{(l)}\right) & =\operatorname{Cov}_{\xi_{2} \mid \xi_{1}}\left[\operatorname{vec}\left(\boldsymbol{U}^{(s)} \mathbf{x}_{s}^{(k)}\right), \operatorname{vec}\left(\boldsymbol{U}^{(s)} \mathbf{x}_{s}^{(l)}\right)\right] \\
& =\operatorname{Cov}_{\xi_{2} \mid \xi_{1}}\left[\left(\mathbf{x}_{s}^{(k)^{\prime}} \otimes \mathbf{I}_{M}\right) \operatorname{vec}\left(\boldsymbol{U}^{(s)}\right),\left(\mathbf{x}_{s}^{(l)^{\prime}} \otimes \mathbf{I}_{M}\right) \operatorname{vec}\left(\boldsymbol{U}^{(s)}\right)\right] \\
& =\left(\mathbf{x}_{s}^{(k)^{\prime}} \otimes \mathbf{I}_{M}\right) \operatorname{Cov}_{\xi_{2} \mid \xi_{1}}\left[\operatorname{vec}\left(\boldsymbol{U}^{(s)}\right), \operatorname{vec}\left(\boldsymbol{U}^{(s)}\right)\right]\left(\mathbf{x}_{s}^{(l)} \otimes \mathbf{I}_{M}\right) \\
& =\frac{1}{M-1}\left(\mathbf{x}_{s}^{(k)^{\prime}} \otimes \mathbf{I}_{M}\right)\left(\mathbf{P}_{M} \otimes \mathbf{P}_{M}\right)\left(\mathbf{x}_{s}^{(l)} \otimes \mathbf{I}_{M}\right) \\
& =\frac{1}{M-1} \mathbf{x}_{s}^{(k)^{\prime}} \mathbf{P}_{M} \mathbf{x}_{s}^{(l)} \otimes \mathbf{P}_{M}
\end{aligned}
$$

Denotando por $\sigma_{s ; k, l}=\frac{1}{M-1} \mathbf{x}_{s}^{(k)^{\prime}} \mathbf{P}_{M} \mathbf{x}_{s}^{(l)} \in \mathbb{R}$ a covariância entre as variáveis $k$ e $l$ dentro do grupo $s$, segue-se que

$$
\operatorname{Cov}_{\xi_{2} \mid \xi_{1}}\left(\boldsymbol{U}^{(s)} \mathbf{x}_{s}^{(k)}, \boldsymbol{U}^{(s)} \mathbf{x}_{s}^{(l)}\right)=\sigma_{s ; k, l} \mathbf{P}_{M}, \quad s=1, \ldots, N .
$$

Além disso, para $s \neq s^{*}$ temos

$\operatorname{Cov}_{\xi_{2} \mid \xi_{1}}\left(U^{(s)} \mathbf{x}_{s}^{(k)}, U^{\left(s^{*}\right)} \mathbf{x}_{s^{*}}^{(l)}\right)=\left(\mathbf{x}_{s}^{(k)^{\prime}} \otimes \mathbf{I}_{M}\right) \operatorname{Cov}{\xi_{2} \mid \xi_{1}}_{1}\left[\operatorname{vec}\left(\boldsymbol{U}^{(s)}\right), \operatorname{vec}\left(\boldsymbol{U}^{\left(s^{*}\right)}\right)\right]\left(\mathbf{x}_{s^{*}}^{(l)} \otimes \mathbf{I}_{M}\right)$.

Como a aleatorização no grupo $s$ é independente da aleatorização no grupo $s^{*}$,

$$
\operatorname{Cov}_{\xi_{2} \mid \xi_{1}}\left[\operatorname{vec}\left(U^{(s)}\right), \operatorname{vec}\left(U^{\left(s^{*}\right)}\right)\right]=0 \quad \forall s \neq s^{*}, \quad s, s^{*}=1, \ldots, N .
$$

Assim,

$$
\operatorname{Cov}_{\xi_{2} \mid \xi_{1}}\left[\left(\underset{s=1}{\oplus} U^{(s)}\right) \mathrm{x}^{(k)},\left(\underset{s=1}{\stackrel{N}{\oplus}} U^{(s)}\right) \mathrm{x}^{(l)}\right]=\bigoplus_{s=1}^{N}\left(\sigma_{s ; k, l} \otimes \mathrm{P}_{M}\right)=\mathrm{D}_{\sigma_{s: k, l}} \otimes \mathrm{P}_{M}
$$

em que

$$
\mathrm{D}_{\sigma_{s: k, l}}=\operatorname{diag}\left\{\sigma_{1: k, l}, \ldots, \sigma_{N: k, l}\right\}
$$

Logo.

$$
\begin{aligned}
\operatorname{Cov}_{\xi_{2} \mid \xi_{1}}\left(X^{(k)}, X^{(l)}\right) & =\left(U \otimes \mathrm{I}_{M I}\right)\left(\mathrm{D}_{\sigma_{s: k, l}} \otimes \mathrm{P}_{. M}\right)\left(U^{\prime} \otimes \mathrm{I}_{M}\right) \\
& =U \mathrm{D}_{\sigma_{* ; k, l}} U^{\prime} \otimes \mathrm{P}_{M}
\end{aligned}
$$

Notemos que $U \mathrm{D}_{\sigma_{s, k \ell l}} U^{\prime}$ é uma matriz diagonal com a posição dos elementos $\sigma_{s: k . l}$ trocada. Portanto,

$$
E_{\xi_{1}}\left[\operatorname{Cov}_{\xi_{2} \mid \xi_{1}}\left(X^{(k)}, X^{(l)}\right)\right]=E_{\xi_{1}}\left(U D_{\sigma_{*: k, l}} U^{\prime}\right) \otimes \mathbf{P}_{M}=\sigma_{e: k, l} \mathrm{I}_{N} \otimes \mathrm{P}_{M}
$$


em que $\sigma_{e ; k, l}=\frac{1}{N} \sum_{s=1}^{N} \sigma_{s ; k, l}$ é a média das covariâncias entre as variáveis $k$ e $l$ dentro dos grupos.

Finalmente, de (C.3) e (C.4) obtemos

$$
\operatorname{Cov}_{\xi_{1} \xi_{2}}\left(\boldsymbol{X}^{(k)}, \boldsymbol{X}^{(l)}\right)=\sigma_{\mu ; k, l}\left(\mathbf{P}_{N} \otimes \mathbf{J}_{M}\right)+\sigma_{e ; k, l} \mathbf{I}_{N} \otimes \mathbf{P}_{M}
$$

\section{C.1.2 Momentos de $Z$}

Com base nas expressões (C.1) e (C.2) obtenidas na seção C.1.1 para $Y$ e $X^{(k)}$, $k=1, \ldots, p$, podemos concluir que os momentos centrados de primeira e segunda ordem de $\boldsymbol{Z}=\left(\begin{array}{c}\boldsymbol{Y} \\ \operatorname{vec}(\boldsymbol{X})\end{array}\right) \in \mathbb{R}^{(p+1) N M}$, são:

$$
E_{\xi_{1} \xi_{2}}(Z)=\left(\mathbf{I}_{p+1} \otimes \mathbf{1}_{N M}\right) \boldsymbol{\mu}
$$

em que $\boldsymbol{\mu}=\left(\mu^{(y)}, \mu^{(1)}, \ldots, \mu^{(p)}\right)^{\prime} \in \mathbb{R}^{p+1}$ é o vetor com as médias gerais cla variável resposta e das $p$ covariáveis, e

$$
\operatorname{Var}_{\xi_{1} \xi_{2}}(Z)==\left(\begin{array}{cc}
\Sigma_{y} & \Sigma_{y, X} \\
\Sigma_{X, y} & \Sigma_{X}
\end{array}\right)=\Sigma_{\mu} \otimes \mathrm{P}_{N} \otimes \mathrm{J}_{M}+\Sigma_{e} \otimes \mathbf{I}_{N} \otimes \mathrm{P}_{M}=\mathrm{V},
$$

em que

$$
\begin{gathered}
\Sigma_{y}=\sigma_{\mu ; y}^{2}\left(\mathbf{P}_{N} \otimes \mathbf{J}_{M}\right)+\sigma_{e ; y}^{2}\left(\mathbf{I}_{N} \otimes \mathbf{P}_{M}\right) \in \mathbb{R}^{N M \times N M} \\
\Sigma_{X, y}=\sigma_{\mu: X, y} \otimes \mathbf{P}_{N} \otimes \mathbf{J}_{M}+\sigma_{e ; X, y} \mathbf{I}_{N} \otimes \mathbf{P}_{M} \in \mathbb{R}^{p N M \times N M}=\Sigma_{y, X}^{\prime}
\end{gathered}
$$

e

$$
\Sigma_{X}=\Sigma_{\mu ; X} \otimes \mathbf{P}_{N} \otimes \mathbf{J}_{M}+\Sigma_{e ; Y} \mathbf{I}_{N} \otimes \mathbf{P}_{M} \in \mathbb{R}^{p N M \times p N M},
$$

com $\sigma_{\mu: X, y}, \sigma_{e: Y, y}, \Sigma_{\mu ; X}, \Sigma_{e ;:}, \Sigma_{\mu}$ e $\Sigma_{e}$ definidas em (4.5), (4.2), (4.6), (4.3), (4.4) e (4.1), respectivamente.

\section{C.1.3 Momentos induzidos para a parte amostrada e não amostrada}

Consideremos um processo de amostragem aleatória com dois estágios sem reposição de uma população de interesse. No primeiro estágio escollhc-se uma amostra de $n$ grupos $(n \leq N)$ e no segundo estágio escolhe-se uma amostra de $m$ unidades 
$(m \leq M)$ em cada um dos grupos anteriormente selecionados. Subdividimos o vetor $Z^{\star}$ em uma parte amostrada (denotada $Z_{S}^{\star}$ ) e outra não amostrada (denotada $Z_{R}^{\star}$ ).

Deste modo,

$$
\left(\begin{array}{c}
Z_{S}^{\star} \\
Z_{R}^{\star}
\end{array}\right)=\left(\begin{array}{l}
\mathrm{R}_{1} \\
\mathrm{R}_{2}
\end{array}\right) Z^{\star}
$$

em que

$$
\begin{aligned}
& \mathbf{R}_{i}=\mathbf{I}_{p+1} \otimes \mathbf{S}_{i}, i=1,2 \\
& \mathbf{S}_{1}=\mathbf{I}_{1 \bullet} \otimes \tilde{\mathbf{I}}_{1 \bullet} \in \mathbb{R}^{n m \times N M} \\
& \mathbf{S}_{2}=\left(\begin{array}{l}
\mathbf{I}_{1} \bullet \tilde{\mathbf{I}}_{2 \bullet} \\
\mathbf{I}_{2 \bullet} \otimes \mathbf{I}_{M}
\end{array}\right) \in \mathbb{R}^{(N M-n m) \times N M}
\end{aligned}
$$

e $\mathbf{I}_{1 \bullet}, \mathbf{I}_{2}$, $\tilde{\mathrm{I}}_{1}$ e $\tilde{\mathbf{I}}_{2 \bullet}$ são obtidos das seguintes partições das matrizes $\mathrm{I}_{N}$ e $\mathbf{I}_{M}$ :

$$
\mathrm{I}_{N}=\left(\begin{array}{ll}
\mathrm{I}_{11} & \mathrm{O}_{12} \\
\mathrm{O}_{21} & \mathrm{I}_{22}
\end{array}\right)=\left(\begin{array}{l}
\mathrm{I}_{1} \bullet \\
\mathbf{I}_{2 \bullet}
\end{array}\right)=\left(\mathrm{I}_{\bullet 1}, \mathrm{I}_{\bullet 2}\right)
$$

e

$$
\mathrm{I}_{M}=\left(\begin{array}{ll}
\tilde{\mathbf{I}}_{11} & \tilde{\mathrm{O}}_{12} \\
\tilde{\mathrm{O}}_{21} & \tilde{\mathbf{I}}_{22}
\end{array}\right)=\left(\begin{array}{l}
\tilde{\mathbf{I}}_{1 \bullet} \\
\tilde{\mathbf{I}}_{2 \bullet}
\end{array}\right)=\left(\tilde{\mathbf{I}}_{\bullet 1}, \tilde{\mathbf{I}}_{\bullet 2}\right)
$$

com

$$
\begin{array}{rlrl}
\mathrm{I}_{11} & =\mathrm{I}_{n} & \mathrm{I}_{1 \bullet}=\left(\mathrm{I}_{n}, 0_{n \times(N-n)}\right)=\mathrm{I}_{\bullet 1}^{\prime} \\
\mathrm{O}_{12} & =0_{n \times(N-n)}=\mathrm{O}_{21}^{\prime} & \mathrm{I}_{2 \bullet}=\left(0_{(N-n) \times n}, \mathrm{I}_{N-n}\right)=\mathrm{I}_{\bullet 2}^{\prime} \\
\mathrm{I}_{22} & =\mathrm{I}_{N-n} & &
\end{array}
$$

e

$$
\begin{aligned}
\tilde{\mathrm{I}}_{11} & =\mathrm{I}_{m} & & \tilde{\mathrm{I}}_{1 \bullet}=\left(\mathrm{I}_{m}, 0_{m \times(M-m)}\right)=\tilde{\mathrm{I}}_{\bullet 1}^{\prime} \\
\tilde{\mathrm{O}}_{12} & =0_{m \times(M-m)}=\tilde{\mathrm{O}}_{21}^{\prime} & & \tilde{\mathrm{I}}_{2 \bullet}=\left(0_{(M-m) \times m}, \mathrm{I}_{M-m}\right)=\tilde{\mathrm{I}}_{\bullet 2}^{\prime} \\
\tilde{\mathbf{I}}_{22} & =\mathrm{I}_{M-m} & &
\end{aligned}
$$

A partir dos momentos de $Z^{\star}$, podemos obter os momentos de $\left(\begin{array}{c}Z_{S}^{\star} \\ Z_{R}^{\star}\end{array}\right)$ como segue:

1) $E\left[\left(\begin{array}{c}Z_{S}^{\star} \\ Z_{R}^{*}\end{array}\right)\right]=\left(\begin{array}{l}\mathrm{R}_{1} \\ \mathrm{R}_{2}\end{array}\right) E\left(Z^{\star}\right)=\left(\begin{array}{l}\mathrm{R}_{1} \\ \mathrm{R}_{2}\end{array}\right) \mathrm{F}^{\star} \mu^{(y)}=\left(\begin{array}{c}\mathrm{F}_{S}^{\star} \\ \mathrm{F}_{R}^{\star}\end{array}\right) \mu^{(y)}$ 
em que

$$
\begin{aligned}
\mathbf{F}_{S}^{\star}=\mathbf{R}_{1} \mathbf{F}^{\star} & =\left(\mathbf{I}_{p+1} \otimes \mathbf{S}_{1}\right)\left(\begin{array}{c}
1_{N M} \\
0_{p N M}
\end{array}\right) \\
& =\left(\begin{array}{cc}
\mathbf{S}_{1} & \mathbf{0}_{n m \times p N M} \\
0_{p n m \times N M} & \mathbf{I}_{p} \otimes \mathbf{S}_{1}
\end{array}\right)\left(\begin{array}{c}
\mathbf{1}_{N} \otimes \mathbf{1}_{M} \\
0_{p N M}
\end{array}\right) \\
& =\left(\begin{array}{c}
\mathbf{I}_{1} 1_{N} \otimes \tilde{\mathbf{I}}_{1 \bullet} 1_{M} \\
0_{p n m}
\end{array}\right) \\
& =\left(\begin{array}{c}
\mathbf{1}_{n} \otimes 1_{m} \\
0_{p m m}
\end{array}\right)
\end{aligned}
$$

e

$$
\begin{aligned}
\mathbf{F}_{R}^{\star}=\mathbf{R}_{2} \mathbf{F}^{\star} & =\left(\mathbf{I}_{p+1} \otimes \mathrm{S}_{2}\right)\left(\begin{array}{c}
\mathbf{1}_{N M} \\
0_{p N M}
\end{array}\right) \\
& =\left(\begin{array}{cc}
\mathbf{S}_{2} & \mathbf{0}_{N M-n m \times p N M} \\
0_{p(N M-n m) \times N M} & \mathrm{I}_{p} \otimes \mathrm{S}_{2}
\end{array}\right)\left(\begin{array}{c}
\mathbf{1}_{N} \otimes \mathbf{1}_{M} \\
0_{p N M}
\end{array}\right) \\
& =\left(\begin{array}{c}
\mathbf{S}_{2}\left(\mathbf{1}_{N} \otimes \mathbf{1}_{M}\right) \\
0_{p(N M-n m)}
\end{array}\right) \\
& =\left(\begin{array}{c}
1_{N M-n m} \\
0_{p(N M-n m)}
\end{array}\right)
\end{aligned}
$$

pois

$$
\begin{aligned}
\mathbf{S}_{2}\left(\mathbf{1}_{N} \otimes \mathbf{1}_{M}\right) & =\left(\begin{array}{l}
\mathbf{I}_{1} \otimes \tilde{\mathbf{I}}_{2 \bullet} \\
\mathbf{I}_{2 \bullet} \otimes \mathbf{I}_{M}
\end{array}\right)\left(\mathbf{1}_{N} \otimes \mathbf{1}_{M}\right)=\left(\begin{array}{l}
\mathbf{I}_{1 \bullet} 1_{N} \otimes \tilde{\mathbf{I}}_{2 \bullet} 1_{M} \\
\mathbf{I}_{2 \bullet} 1_{N} \otimes \mathbf{I}_{M} 1_{M}
\end{array}\right)=\left(\begin{array}{l}
\mathbf{1}_{n} \otimes \mathbf{1}_{M-m} \\
1_{N-n} \otimes \mathbf{1}_{M}
\end{array}\right) \\
& =1_{N M-n m}
\end{aligned}
$$

2)

$$
\begin{aligned}
\operatorname{Var}_{\xi_{1} \xi_{2}}\left[\left(\begin{array}{c}
Z_{S}^{\star} \\
Z_{R}^{\star}
\end{array}\right)\right] & =\left(\begin{array}{cc}
\operatorname{Cov}_{\xi_{1} \xi_{2}}\left(Z_{S}^{\star}, Z_{S}^{\star}\right) & \operatorname{Cov}_{\xi_{1} \xi_{2}}\left(Z_{S}^{\star}, Z_{R}^{\star}\right) \\
\operatorname{Cov}_{\xi_{1} \xi_{2}}\left(Z_{R}^{\star}, Z_{S}^{\star}\right) & \operatorname{Cov}_{\xi_{1} \xi_{2}}\left(Z_{R}^{\star}, Z_{R}^{\star}\right)
\end{array}\right) \\
& =\left(\begin{array}{cc}
\mathrm{V}_{S} & \mathrm{~V}_{S R} \\
\mathrm{~V}_{R S} & \mathrm{~V}_{R}
\end{array}\right) \\
& =\left(\begin{array}{ll}
\mathrm{R}_{1} \mathrm{VR} & \mathrm{R}_{1}^{\prime} \mathrm{VR}_{2}^{\prime} \\
\mathrm{R}_{2} \mathrm{~V} \mathrm{R}_{1}^{\prime} & \mathrm{R}_{2} \mathrm{VR}_{2}^{\prime}
\end{array}\right)
\end{aligned}
$$


Para facilitar o cálculo de cada componente, consideremos as seguintes partições de $\mathbf{P}_{N}, \mathbf{P}_{M}$ e $\mathbf{J}_{M}$ :

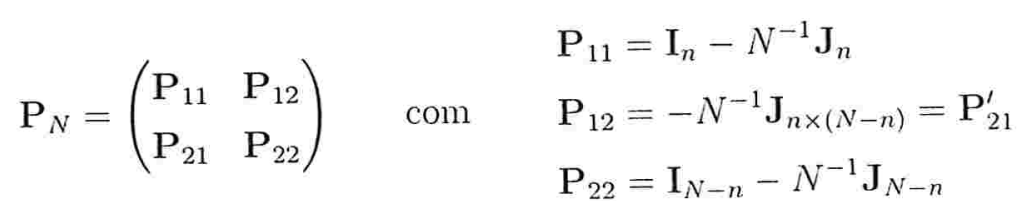

sendo $\mathrm{J}_{a \times b}=1_{a} 1_{b}^{\prime}$

$$
\mathbf{J}_{M}=\left(\begin{array}{ll}
\tilde{\mathbf{J}}_{11} & \tilde{\mathbf{J}}_{12} \\
\tilde{\mathbf{J}}_{21} & \tilde{\mathbf{J}}_{22}
\end{array}\right)=\left(\begin{array}{l}
\tilde{\mathbf{J}}_{1 \bullet} \\
\tilde{\mathbf{J}}_{2 \bullet}
\end{array}\right)=\left(\tilde{\mathbf{J}}_{\bullet 1}, \tilde{\mathbf{J}}_{\bullet 2}\right)
$$

com

$$
\begin{array}{ll}
\tilde{\mathbf{J}}_{11}=\mathbf{J}_{m} & \tilde{\mathbf{J}}_{1 \bullet}=\mathbf{J}_{m \times M}=\tilde{\mathbf{J}}_{\bullet 1}^{\prime} \\
\tilde{\mathbf{J}}_{12}=\mathbf{J}_{m \times(M-m)}=\tilde{\mathbf{J}}_{21}^{\prime} & \tilde{\mathbf{J}}_{2 \bullet}=\mathbf{J}_{(M-m) \times M}=\tilde{\mathbf{J}}_{\bullet 2}^{\prime} \\
\tilde{\mathbf{J}}_{22}=\mathbf{J}_{M-m} &
\end{array}
$$

e

$$
\mathbf{P}_{M}=\left(\begin{array}{ll}
\tilde{\mathbf{P}}_{11} & \tilde{\mathbf{P}}_{12} \\
\tilde{\mathbf{P}}_{21} & \tilde{\mathbf{P}}_{22}
\end{array}\right)=\left(\begin{array}{c}
\tilde{\mathbf{P}}_{1 \bullet} \\
\tilde{\mathbf{P}}_{2 \bullet}
\end{array}\right)=\left(\tilde{\mathbf{P}}_{\bullet 1}, \tilde{\mathbf{P}}_{\bullet 2}\right),
$$

com

$$
\begin{array}{ll}
\tilde{\mathbf{P}}_{11}=\mathrm{I}_{m}-M^{-1} \mathbf{J}_{m} & \tilde{\mathbf{P}}_{1 \bullet}=\left(\tilde{\mathbf{P}}_{11}, \tilde{\mathbf{P}}_{12}\right)=\tilde{\mathbf{I}}_{1 \bullet}-M^{-1} \tilde{\mathbf{J}}_{1 \bullet}=\tilde{\mathbf{P}}_{\bullet 1}^{\prime} \\
\tilde{\mathbf{P}}_{12}=-M^{-1} \mathbf{J}_{m \times(M-m)}=\tilde{\mathbf{P}}_{21}^{\prime} & \tilde{\mathbf{P}}_{2 \bullet}=\left(\tilde{\mathbf{P}}_{21}, \tilde{\mathbf{P}}_{22}\right)=\tilde{\mathbf{I}}_{2 \bullet}-M^{-1} \tilde{\mathbf{J}}_{2 \bullet}=\tilde{\mathbf{P}}_{\bullet 2}^{\prime} \\
\tilde{\mathbf{P}}_{22}=\mathrm{I}_{M-m}-M^{-1} \mathbf{J}_{M-m} &
\end{array}
$$

Calculemos agora cada componente de $\operatorname{Var}_{\xi_{1} \xi_{2}}\left[\left(\begin{array}{c}Z_{S}^{\star} \\ Z_{R}^{\star}\end{array}\right)\right]$

a)

$$
\begin{aligned}
\mathrm{V}_{S} & =\mathbf{R}_{1} \mathbf{V} \mathbf{R}_{1}^{\prime} \\
& =\left(\mathbf{I}_{p+1} \otimes \mathrm{S}_{1}\right)\left(\Sigma_{\mu} \otimes \mathrm{P}_{N} \otimes \mathbf{J}_{M}+\Sigma_{e} \otimes \mathbf{I}_{N} \otimes \mathbf{P}_{M}\right)\left(\mathbf{I}_{p+1} \otimes \mathrm{S}_{1}^{\prime}\right) \\
& =\Sigma_{\mu} \otimes \mathrm{S}_{1}\left(\mathrm{P}_{N} \otimes \mathbf{J}_{M}\right) \mathrm{S}_{1}^{\prime}+\Sigma_{e} \otimes \mathrm{S}_{1}\left(\mathbf{I}_{N} \otimes \mathrm{P}_{M}\right) \mathrm{S}_{1}^{\prime}
\end{aligned}
$$

com

$\mathrm{S}_{1}\left(\mathrm{P}_{N} \odot \mathbf{J}_{M}\right) \mathrm{S}_{1}^{\prime}=\left(\mathrm{I}_{1 \bullet} \odot \tilde{\mathbf{I}}_{1 \bullet}\right)\left(\mathrm{P}_{N} \otimes \mathbf{J}_{M}\right)\left(\mathrm{I}_{\bullet 1} \odot \tilde{\mathbf{I}}_{\bullet 1}\right)=\mathrm{I}_{1 \bullet} \cdot \mathrm{P}_{X} \mathrm{I}_{\bullet 1} \otimes \tilde{\mathbf{I}}_{1 \bullet} \mathbf{J}_{M} \tilde{\mathbf{I}}_{\bullet 1}=\mathrm{P}_{11} \odot \tilde{\mathbf{J}}_{11}$

$\mathrm{e}$

$$
\mathrm{S}_{1}\left(\mathrm{I}_{N} \otimes \mathrm{P}_{M}\right) \mathrm{S}_{1}^{\prime}=\mathrm{I}_{1 \bullet} \mathrm{I}_{N} \mathrm{I}_{\bullet 1} \otimes \tilde{\mathrm{I}}_{1 \bullet} \mathrm{P}_{M I} \tilde{\mathrm{I}}_{\bullet 1}=\mathrm{I}_{11} \otimes \tilde{\mathrm{P}}_{11} .
$$


Portanto

$$
\mathbf{V}_{S}=\Sigma_{\mu} \otimes\left(\mathbf{I}_{n}-N^{-1} \mathbf{J}_{n}\right) \otimes \mathbf{J}_{m}+\Sigma_{e} \otimes \mathbf{I}_{n} \otimes\left(\mathbf{I}_{m}-M^{-1} \mathbf{J}_{m}\right) .
$$

b)

com

$$
\begin{aligned}
\mathbf{V}_{S R} & =\mathbf{R}_{1} \mathbf{V} \mathbf{R}_{2}^{\prime} \\
& =\left(\mathbf{I}_{p+1} \otimes \mathbf{S}_{1}\right)\left(\Sigma_{\mu} \otimes \mathbf{P}_{N} \otimes \mathbf{J}_{M}+\Sigma_{e} \otimes \mathbf{I}_{N} \otimes \mathbf{P}_{M}\right)\left(\mathbf{I}_{p+1} \otimes \mathbf{S}_{2}^{\prime}\right) \\
& =\Sigma_{\mu} \otimes \mathbf{S}_{1}\left(\mathbf{P}_{N} \otimes \mathbf{J}_{M}\right) \mathbf{S}_{2}^{\prime}+\Sigma_{e} \otimes \mathbf{S}_{1}\left(\mathbf{I}_{N} \otimes \mathbf{P}_{M}\right) \mathbf{S}_{2}^{\prime} \\
& =\mathbf{V}_{R S}^{\prime}
\end{aligned}
$$

$$
\begin{aligned}
\mathbf{S}_{1}\left(\mathbf{P}_{N} \otimes \mathbf{J}_{M}\right) \mathbf{S}_{2}^{\prime} & =\left(\mathbf{I}_{1} \bullet \tilde{\mathbf{I}}_{1 \bullet}\right)\left(\mathbf{P}_{N} \otimes \mathbf{J}_{M}\right)\left(\mathbf{I}_{\bullet 1} \otimes \tilde{\mathbf{I}}_{\bullet 2}, \mathbf{I}_{\bullet 2} \otimes \mathbf{I}_{M}\right) \\
& =\left(\mathbf{I}_{1} \otimes \tilde{\mathbf{I}}_{1 \bullet}\right)\left(\left(\mathbf{P}_{N} \otimes \mathbf{J}_{M}\right)\left(\mathbf{I}_{\bullet 1} \otimes \tilde{\mathbf{I}}_{\bullet 2}\right),\left(\mathbf{P}_{N} \otimes \mathbf{J}_{M}\right)\left(\mathbf{I}_{\bullet 2} \otimes \mathbf{I}_{M}\right)\right) \\
& =\left(\mathbf{I}_{1} \otimes \tilde{\mathbf{I}}_{1 \bullet}\right)\left(\mathbf{P}_{N} \mathbf{I}_{\bullet 1} \otimes \mathbf{J}_{M} \tilde{\mathbf{I}}_{\bullet 2}, \mathbf{P}_{N} \mathbf{I}_{\bullet 2} \otimes \mathbf{J}_{M} \mathbf{I}_{M}\right) \\
& =\left(\mathbf{I}_{1} \bullet \mathbf{P}_{N} \mathbf{I}_{\bullet 1} \otimes \tilde{\mathbf{I}}_{1 \bullet} \mathbf{J}_{M} \tilde{\mathbf{I}}_{\bullet 2}, \mathbf{I}_{1 \bullet} \mathbf{P}_{N} \mathbf{I}_{\bullet 2} \otimes \tilde{\mathbf{I}}_{1 \bullet} \mathbf{J}_{M} \mathbf{I}_{M}\right) \\
& =\left(\mathbf{P}_{11} \otimes \tilde{\mathbf{J}}_{12}, \mathbf{P}_{12} \otimes \tilde{\mathbf{J}}_{1 \bullet}\right)
\end{aligned}
$$

$\mathrm{e}$

$$
\begin{aligned}
\mathbf{S}_{1}\left(\mathbf{I}_{N} \otimes \mathbf{P}_{M}\right) \mathbf{S}_{2}^{\prime} & =\left(\mathbf{I}_{1 \bullet} \otimes \tilde{\mathbf{I}}_{1 \bullet}\right)\left(\mathbf{I}_{N} \otimes \mathbf{P}_{M}\right)\left(\mathbf{I}_{\bullet 1} \otimes \tilde{\mathbf{I}}_{\bullet 2}, \mathbf{I}_{\bullet 2} \otimes \mathbf{I}_{M}\right) \\
& =\left(\mathbf{I}_{1 \bullet} \mathbf{I}_{N} \mathbf{I}_{\bullet 1} \otimes \tilde{\mathbf{I}}_{1 \bullet} \mathbf{P}_{M} \tilde{\mathbf{I}}_{\bullet 2}, \mathbf{I}_{1 \bullet} \mathbf{I}_{N} \mathbf{I}_{\bullet 2} \otimes \tilde{\mathbf{I}}_{1 \bullet} \cdot \mathbf{P}_{M} \mathbf{I}_{M}\right) \\
& =\left(\mathbf{I}_{11} \otimes \tilde{\mathbf{P}}_{12}, \mathrm{O}_{12} \otimes \tilde{\mathbf{P}}_{1 \bullet}\right)
\end{aligned}
$$

Note que

$$
\mathbf{P}_{12} \otimes \tilde{\mathbf{J}}_{1 \bullet}=-N^{-1} \mathbf{J}_{n \times(N-n)} \otimes \mathbf{J}_{m \times M}=-N^{-1} \mathbf{J}_{n m \times(N-n) M}
$$

e

$$
\mathrm{O}_{12} \otimes \tilde{\mathbf{P}}_{1 \bullet}=0_{n \times(N-n)} \otimes\left(\mathbf{I}_{m}-M^{-1} \mathbf{J}_{m},-M^{-1} \mathbf{J}_{m \times(M-m)}\right)=0_{n m \times(N-n) M}
$$

de modo que

$$
\begin{aligned}
\mathrm{V}_{S R} & =\Sigma_{\mu} \otimes\left(\left(\mathbf{I}_{n}-N^{-1} \mathbf{J}_{n}\right) \otimes \mathbf{J}_{m \times(M-m)},-N^{-1} \mathbf{J}_{n m \times(N-n) M}\right) \\
& -M^{-1} \Sigma_{e} \otimes\left(\mathrm{I}_{n} \otimes \mathbf{J}_{m \times(M-m)}, 0_{n m \times(N-n) . M}\right) \\
& =\left(\Sigma_{\mu}-M^{-1} \Sigma_{e}\right) \otimes\left(\mathrm{I}_{n} \otimes \mathbf{J}_{m \times(M-m)}, 0_{m n \times(N-n) M}\right) \\
& -N^{-1} \Sigma_{\mu} \otimes \mathbf{J}_{n m \times(N-n) M .} .
\end{aligned}
$$


c)

$$
\begin{aligned}
\mathrm{V}_{R} & =\mathbf{R}_{2} \mathbf{V} \mathbf{R}_{2}^{\prime} \\
& =\left(\mathbf{I}_{p+1} \otimes \mathrm{S}_{2}\right)\left(\Sigma_{\mu} \otimes \mathbf{P}_{N} \otimes \mathbf{J}_{M}+\Sigma_{e} \otimes \mathbf{I}_{N} \otimes \mathbf{P}_{M}\right)\left(\mathbf{I}_{p+1} \otimes \mathbf{S}_{2}^{\prime}\right) \\
& =\Sigma_{\mu} \otimes \mathbf{S}_{2}\left(\mathbf{P}_{N} \otimes \mathbf{J}_{M}\right) \mathrm{S}_{2}^{\prime}+\Sigma_{e} \otimes \mathbf{S}_{2}\left(\mathbf{I}_{N} \otimes \mathbf{P}_{M}\right) \mathbf{S}_{2}^{\prime}
\end{aligned}
$$

com

$$
\begin{aligned}
& \mathrm{S}_{2}\left(\mathrm{P}_{N} \otimes \mathrm{J}_{M}\right) \mathrm{S}_{2}^{\prime}=\left(\begin{array}{c}
\mathrm{I}_{1} \bullet \tilde{\mathrm{I}}_{2 \bullet} \\
\mathrm{I}_{2 \bullet} \otimes \mathrm{I}_{M}
\end{array}\right)\left(\mathrm{P}_{N} \otimes \mathrm{J}_{M}\right)\left(\mathrm{I}_{\bullet 1} \otimes \tilde{\mathrm{I}}_{\bullet 2}, \mathrm{I}_{\bullet 2} \otimes \mathrm{I}_{M}\right) \\
& =\left(\begin{array}{ll}
\mathrm{I}_{1} \bullet P_{N} \mathbf{I}_{\bullet 1} \otimes \tilde{\mathrm{I}}_{2 \bullet} \mathbf{J}_{M} \tilde{\mathbf{I}}_{\bullet 2} & \mathrm{I}_{1} \cdot \mathbf{P}_{N} \mathbf{I}_{\bullet 2} \otimes \tilde{\mathbf{I}}_{2 \bullet} \mathbf{J}_{M} \mathbf{I}_{M} \\
\mathbf{I}_{2} \cdot \mathbf{P}_{N} \mathbf{I}_{\bullet 1} \otimes \mathbf{I}_{M} \mathbf{J}_{M} \tilde{\mathbf{I}}_{\bullet 2} & \mathbf{I}_{2 \bullet} \mathbf{P}_{N} \mathbf{I}_{\bullet 2} \otimes \mathbf{I}_{M} \mathbf{J}_{M} \mathbf{I}_{M}
\end{array}\right) \\
& =\left(\begin{array}{ll}
\mathbf{P}_{11} \otimes \tilde{\mathbf{J}}_{22} & \mathbf{P}_{12} \otimes \tilde{\mathbf{J}}_{2 \bullet} \\
\mathbf{P}_{21} \otimes \tilde{\mathbf{J}}_{\bullet 2} & \mathbf{P}_{22} \otimes \mathbf{J}_{M}
\end{array}\right)
\end{aligned}
$$

e

$$
\begin{aligned}
& \mathrm{S}_{2}\left(\mathrm{I}_{N} \otimes \mathrm{P}_{M}\right) \mathrm{S}_{2}^{\prime}=\left(\begin{array}{c}
\mathrm{I}_{1} \otimes \tilde{\mathrm{I}}_{2 \bullet} \\
\mathrm{I}_{2 \bullet} \otimes \mathrm{I}_{M}
\end{array}\right)\left(\mathrm{I}_{N} \otimes \mathrm{P}_{M}\right)\left(\mathrm{I}_{\bullet 1} \otimes \tilde{\mathrm{I}}_{\bullet 2}, \mathrm{I}_{\bullet 2} \otimes \mathrm{I}_{M}\right) \\
& =\left(\begin{array}{ll}
\mathbf{I}_{1} \cdot \mathbf{I}_{N} \mathbf{I}_{\bullet 1} \otimes \tilde{\mathbf{I}}_{2 \bullet} \mathbf{P}_{M} \tilde{\mathbf{I}}_{\bullet 2} & \mathbf{I}_{\mathbf{\bullet}} \mathbf{I}_{N} \mathbf{I}_{\bullet 2} \otimes \tilde{\mathbf{I}}_{2 \bullet} \mathbf{P}_{M} \mathbf{I}_{M} \\
\mathbf{I}_{2} \bullet \mathbf{I}_{N} \mathbf{I}_{\bullet 1} \otimes \mathbf{I}_{M} \mathbf{P}_{M} \tilde{\mathbf{I}}_{\bullet 2} & \mathbf{I}_{2} \bullet \mathbf{I}_{N} \mathbf{I}_{\bullet 2} \otimes \mathbf{I}_{M} \mathbf{P}_{M} \mathrm{I}_{M}
\end{array}\right) \\
& =\left(\begin{array}{ll}
\mathbf{I}_{11} \otimes \tilde{\mathbf{P}}_{22} & \mathrm{O}_{12} \otimes \tilde{\mathbf{P}}_{2 \bullet} \\
\mathrm{O}_{21} \otimes \tilde{\mathbf{P}}_{\bullet 2} & \mathrm{I}_{22} \otimes \mathrm{P}_{M}
\end{array}\right)
\end{aligned}
$$

Note que

$$
\begin{aligned}
\mathbf{S}_{2}\left(\mathbf{P}_{N} \otimes \mathbf{J}_{M}\right) \mathbf{S}_{2}^{\prime} & =\left(\begin{array}{cc}
\left(\mathbf{I}_{n}-N^{-1} \mathbf{J}_{n}\right) \otimes \mathbf{J}_{M-m} & -N^{-1} \mathbf{J}_{n(M-m) \times(N-n) M} \\
-N^{-1} \mathbf{J}_{(N-n) M \times n(M-m)} & \left(\mathbf{I}_{N-n}-N^{-1} \mathbf{J}_{N-n}\right) \otimes \mathbf{J}_{M}
\end{array}\right) \\
& =\left(\begin{array}{cc}
\mathbf{I}_{n} \otimes \mathbf{J}_{M-m} & 0_{n(M-m) \times(N-n) M} \\
\mathbf{O}_{(N-n) . M \times n(M-m)} & \mathbf{I}_{N-n} \otimes \mathbf{J}_{M}
\end{array}\right)-N^{-1} \mathbf{J}_{N M-n m}
\end{aligned}
$$

e

$$
\begin{aligned}
& \mathbf{S}_{2}\left(\mathbf{I}_{N} \otimes \mathbf{P}_{M}\right) \mathrm{S}_{2}^{\prime}=\left(\begin{array}{cc}
\mathrm{I}_{n} \otimes\left(\mathrm{I}_{M-m}-M^{-1} \mathrm{~J}_{. M-m}\right) & 0_{n(M-m) \times(N-n) . M} \\
0_{(N-n) . M \times n(M-m)} & \mathrm{I}_{M-n} \otimes\left(\mathbf{I}_{M}-. I^{-1} \mathrm{~J}_{M}\right)
\end{array}\right) \\
& =\left(\begin{array}{cc}
\mathbf{I}_{n(M-m)}-M^{-1} \mathbf{I}_{n} \otimes \mathbf{J}_{M-m} & 0_{n(M-m) \times(N-n) . M} \\
0_{(N-n) M \times n(M-m)} & \mathbf{I}_{(N-n) . M}-. M^{-1} \mathbf{I}_{N-n} \otimes \mathbf{J}_{M}
\end{array}\right) \\
& =\mathrm{I}_{N, M-n m}-M^{-1}\left(\begin{array}{cc}
\mathrm{I}_{n} \otimes \mathbf{J}_{. M-m} & 0_{n(M-m) \times(N-n) M} \\
\mathbf{O}_{(N-n) . M \times n(. Y-m)} & \mathrm{I}_{N-n} \otimes \mathbf{J}_{M}
\end{array}\right) .
\end{aligned}
$$


$\underline{\text { C.1 Estimadores e preditores ótimos em presença de covariáveis - Detalhes }}$

portanto,

$$
\begin{aligned}
\mathrm{V}_{R} & =\Sigma_{e} \otimes \mathrm{I}_{N M-n m}+\left(\Sigma_{\mu}-M^{-1} \Sigma_{e}\right) \otimes\left(\begin{array}{cc}
\mathbf{I}_{n} \otimes \mathbf{J}_{M-m} & 0_{n(M-m) \times(N-n) M} \\
0_{(N-n) M \times n(M-m)} & \mathbf{I}_{N-n} \otimes \mathbf{J}_{M}
\end{array}\right) \\
& -N^{-1} \Sigma_{\mu} \otimes \mathbf{J}_{N M-n m} .
\end{aligned}
$$

C.1.4 Predição dos valores latentes dos grupos amostrados usando informação das covariáveis

Calculemos cada componente de

$$
\widehat{\theta}^{(y)}=\mathbf{L}_{S}^{\prime} Z_{S}^{\star}+\mathbf{L}_{R}^{\prime}\left[\mathbf{F}_{R}^{\star} \widehat{\mu}^{(y)}+\mathrm{V}_{R S} \mathbf{V}_{S}^{-1}\left(Z_{S}^{\star}-\mathbf{F}_{S}^{\star} \widehat{\mu}^{(y)}\right)\right]
$$

1)

$$
\begin{aligned}
\mathbf{L}_{S}^{\prime} Z_{S}^{\star} & =\left(\mathbf{I}_{n} \otimes M^{-1} 1_{m}^{\prime}, \mathbf{0}_{n \times p n m}\right)\left(\begin{array}{c}
Y_{S} \\
v e c\left(X_{S}\right)-\mu^{(X)} \otimes 1_{n m}
\end{array}\right) \\
& =\frac{m}{M} \bar{Y}_{S}
\end{aligned}
$$

em que

$$
\overline{\boldsymbol{Y}}_{S}=\left(\mathbf{I}_{n} \otimes m^{-1} 1_{m}^{\prime}\right) \boldsymbol{Y}_{S}
$$

2)

$$
\begin{aligned}
\mathrm{L}_{R}^{\prime} \mathbf{F}_{R}^{\star} \widehat{\mu}^{(y)} & =\left(\mathrm{I}_{n} \otimes M^{-1} 1_{M-m}^{\prime}, 0_{n \times(N-n) M}: 0_{n \times p(N M-n m)}\right)\left(\begin{array}{c}
1_{n(M-m)} \\
1_{(N-n) M} \\
0_{p(N M-n m)}
\end{array}\right) \widehat{\mu}^{(y)} \\
& =\left(\frac{M-m}{M}\right) \widehat{\mu}^{(y)} 1_{n}
\end{aligned}
$$


3) Observando que

$$
\begin{aligned}
\mathrm{V}_{R S} & =\left(\Sigma_{\mu}-M^{-1} \Sigma_{e}\right) \otimes\left(\begin{array}{c}
\mathbf{I}_{n} \otimes \mathbf{J}_{(M-m) \times m} \\
0_{(N-n) M \times n m}
\end{array}\right)-N^{-1} \Sigma_{\mu} \otimes \mathrm{J}_{(N M-n m) \times n m} \\
& =\Sigma^{*} \otimes\left(\begin{array}{c}
\mathbf{I}_{n} \otimes \mathbf{J}_{(M-m) \times m} \\
0_{(N-n) M \times n m}
\end{array}\right)-N^{-1} \Sigma_{\mu} \otimes\left(\begin{array}{c}
\mathrm{J}_{n(M-m) \times n m} \\
\mathrm{~J}_{(N-n) M \times n m}
\end{array}\right) \\
& =\left(\begin{array}{cc}
\sigma_{y}^{* 2} & \sigma_{y, X}^{*} \\
\sigma_{X, y}^{*} & \Sigma_{X}^{*}
\end{array}\right) \otimes\left(\begin{array}{c}
\mathrm{I}_{n} \otimes \mathbf{J}_{(M-m) \times m} \\
0_{(N-n) M \times n m}
\end{array}\right) \\
& -N^{-1}\left(\begin{array}{cc}
\sigma_{\mu ; y}^{2} & \sigma_{\mu ; y, X} \\
\sigma_{\mu ; X ; y} & \Sigma_{\mu ; X}
\end{array}\right) \otimes\left(\begin{array}{c}
\mathrm{J}_{n(M-m) \times n m} \\
\mathrm{~J}_{(N-n) M \times n m}
\end{array}\right) \\
& =\left(\begin{array}{cc}
\mathrm{V}_{R S ; y} & \mathrm{~V}_{R S ; y, X} \\
\mathbf{V}_{R S ; X, y} & \mathrm{~V}_{R S ; X}
\end{array}\right)
\end{aligned}
$$

com

$$
\begin{aligned}
\sigma_{y}^{* 2} & =\sigma_{\mu ; y}^{2}-M^{-1} \sigma_{e ; y}^{2} \\
\sigma_{y, X}^{*} & =\sigma_{\mu ; y, X}-M^{-1} \sigma_{e ; y, X}=\sigma_{X, y}^{*^{\prime}} \\
\Sigma_{X}^{*} & =\Sigma_{\mu ; X}-M^{-1} \Sigma_{e ; X}
\end{aligned}
$$

podemos concluir que

$$
\mathrm{L}_{R}^{\prime} \mathrm{V}_{R S}=\left(\mathrm{A}_{11}, \mathrm{~A}_{12}\right)
$$

com

$$
\begin{aligned}
\mathbf{A}_{11} & =\left(\mathbf{I}_{n} \otimes M^{-1} \mathbf{1}_{M-m}^{\prime}, 0_{n \times(N-n) M}\right) \mathbf{V}_{R S ; y} \\
& =\left(\mathbf{I}_{n} \otimes M^{-1} \mathbf{1}_{M-m}^{\prime}\right)\left[\sigma_{y}^{* 2}\left(\mathbf{I}_{n} \otimes \mathbf{J}_{(M-m) \times m}\right)-N^{-1} \sigma_{\mu ; y}^{2}\left(\mathbf{J}_{n} \otimes \mathbf{J}_{(M-m) \times m}\right)\right] \\
& =\left(\frac{M-m}{M}\right)\left[\sigma_{y}^{* 2}\left(\mathbf{I}_{n} \otimes \mathbf{1}_{m}^{\prime}\right)-N^{-1} \sigma_{\mu: y}^{2} \mathbf{J}_{n} \otimes 1_{m}^{\prime}\right] \\
& =(1-f)\left(\sigma_{y}^{* 2} \mathbf{I}_{n}-N^{-1} \sigma_{\mu: y}^{2} \mathbf{J}_{n}\right) \otimes \mathbf{1}_{m}^{\prime} \\
& =(1-f) \mathbf{B}_{11}
\end{aligned}
$$


e

$$
\begin{aligned}
\mathbf{A}_{12} & =\left(\mathbf{I}_{n} \otimes M^{-1} \mathbf{1}_{M-m}^{\prime}, \mathbf{0}_{n \times(N-n) M}\right) \mathrm{V}_{R S ; y, X} \\
& =\sigma_{y, X}^{*} \otimes\left[\left(\mathbf{I}_{n} \otimes M^{-1} 1_{M-m}^{\prime}, 0_{n \times(N-n) M}\right)\left(\begin{array}{c}
\mathbf{I}_{n} \otimes \mathbf{J}_{(M-m) \times m} \\
0_{(N-n) M \times n m}
\end{array}\right)\right] \\
& -N^{-1} \sigma_{\mu ; y, X} \otimes\left[\left(\mathbf{I}_{n} \otimes M^{-1} \mathbf{1}_{M-m}^{\prime}, 0_{n \times(N-n) M}\right)\left(\begin{array}{l}
\mathbf{J}_{n(M-m) \times n m} \\
\mathbf{J}_{(N-n) M \times n m}
\end{array}\right)\right] \\
& =\left(\frac{M-m}{M}\right) \sigma_{y, X}^{*} \otimes \mathbf{I}_{n} \otimes \mathbf{1}_{m}^{\prime}-N^{-1} \sigma_{\mu ; y, X} \otimes \mathbf{J}_{n} \otimes 1_{m}^{\prime} \\
& =(1-f)\left(\sigma_{y, X}^{*} \otimes \mathbf{I}_{n}-N^{-1} \sigma_{\mu ; y, X} \otimes \mathbf{J}_{n}\right) \otimes \mathbf{1}_{m}^{\prime} \\
& =(1-f) \mathbf{B}_{12}
\end{aligned}
$$

Por outro lado,

$$
\begin{aligned}
\mathrm{V}_{S}^{-1}\left(Z_{S}^{\star}-\mathbf{F}_{S}^{\star} \widehat{\mu}^{(y)}\right) & =\left(\begin{array}{ll}
\Psi_{11} & \Psi_{12} \\
\Psi_{21} & \Psi_{22}
\end{array}\right)\left(\begin{array}{c}
Y_{S}-\widehat{\mu}^{(y)} 1_{n m} \\
\operatorname{vec}\left(X_{S}\right)-\mu^{(X)} \otimes 1_{n m}
\end{array}\right) \\
& =\left(\begin{array}{c}
\Psi_{11}\left(Y_{S}-\widehat{\mu}^{(y)} 1_{n m}\right)+\Psi_{12}\left(\operatorname{vec}\left(X_{S}\right)-\mu^{(X)} \otimes 1_{n m}\right) \\
\Psi_{21}\left(Y_{S}-\widehat{\mu}^{(y)} 1_{n m}\right)+\Psi_{22}\left(\operatorname{vec}\left(X_{S}\right)-\mu^{(X)} \otimes 1_{n m}\right)
\end{array}\right)
\end{aligned}
$$

Portanto,

$$
\begin{aligned}
\mathrm{L}_{R}^{\prime} & \mathrm{V}_{R S} \mathrm{~V}_{S}^{-1}\left(Z_{S}^{\star}-\mathrm{F}_{S}^{\star} \widehat{\mu}^{(y)}\right)= \\
& =\mathrm{A}_{11}\left[\Psi_{11}\left(Y_{S}-\widehat{\mu}^{(y)} 1_{n m}\right)+\Psi_{12}\left(\operatorname{vec}\left(X_{S}\right)-\mu^{(X)} \otimes 1_{n m}\right)\right] \\
& +\mathrm{A}_{12}\left[\Psi_{21}\left(Y_{S}-\widehat{\mu}^{(y)} 1_{n m}\right)+\Psi_{22}\left(\operatorname{vec}\left(X_{S}\right)-\mu^{(X)} \otimes 1_{n m}\right)\right] \\
& +(1-f)\left[\mathrm{B}_{11} \Psi_{11}+\mathrm{B}_{12} \Psi_{21}\right]\left(Y_{S}-\widehat{\mu}^{(y)} 1_{n m}\right) \\
& +(1-f)\left[\mathrm{B}_{11} \Psi_{12}+\mathrm{B}_{12} \Psi_{22}\right]\left(\operatorname{vec}\left(X_{S}\right)-\mu^{(X)} \otimes 1_{n m}\right)
\end{aligned}
$$

Assim. podemos concluir que

$$
\begin{aligned}
\hat{\theta}^{(y)} & =\mathrm{L}_{S}^{\prime} Z_{S}^{\star}+\mathrm{L}_{R}^{\prime}\left[\mathrm{F}_{R}^{*} \widehat{\mu}^{(y)}+\mathrm{V}_{R S} \mathrm{~V}_{S}^{-1}\left(Z_{S}^{\star}-\mathrm{F}_{S}^{*} \widehat{\mu}^{(y)}\right)\right] \\
& =f \bar{Y}_{S}+(1-f) \widehat{\mu}^{(y)} 1_{n} \\
& +(1-f)\left[\mathrm{B}_{11} \Psi_{11}+\mathrm{B}_{12} \Psi_{21}\right]\left(Y_{S}-\widehat{\mu}^{(y)} 1_{n m}\right) \\
& +(1-f)\left[\mathrm{B}_{11} \Psi_{12}+\mathrm{B}_{12} \Psi_{22}\right]\left(\operatorname{vec}\left(X_{S}\right)-\mu^{(N)} \odot 1_{n m}\right)
\end{aligned}
$$




\section{C.2 Amostragem estratificada}

\section{C.2.1 Momentos de $Z_{s}$ e $Z$}

Para o estrato $s, s=1, \ldots, N$ como

$$
E_{\xi_{1}}\left(U^{(s)}\right)=M_{s}^{-1} J_{M_{s}}
$$

temos que

$$
E_{\xi_{1}}\left(Z_{s}\right)=E\left[\left(\mathbf{I}_{p+1} \otimes U^{(s)}\right) \mathbf{z}_{s}\right]=\left(\mathbf{I}_{p+1} \otimes M_{s}^{-1} \mathbf{J}_{M_{s}}\right) \mathbf{z}_{s}=\left(\mathbf{I}_{p+1} \otimes 1_{M_{s}}\right) \mu_{s}
$$

$$
E_{\xi_{1}}(Z)=E\left\{\left[\stackrel{N}{\oplus}\left(\mathbf{I}_{p+1} \otimes U^{(s)}\right)\right] \mathrm{z}\right\}=\left[\bigoplus_{s=1}^{N}\left(\mathbf{I}_{p+1} \otimes 1_{M_{s}}\right)\right] \boldsymbol{\mu}
$$

Por outro lado, como

$$
\operatorname{Cov}_{\xi_{1}}\left(\operatorname{vec}\left(U^{(s)}\right)\right)=\left(M_{s}-1\right)^{-1} \mathbf{P}_{M_{s}} \otimes \mathbf{P}_{M_{s}}
$$

e observando que

$$
\left(\mathbf{I}_{p+1} \otimes U^{(s)}\right) \mathbf{z}_{s}=\left(\begin{array}{c}
U^{(s)} \mathrm{y}_{s} \\
U^{(s)} \mathbf{x}_{s}^{(1)} \\
\vdots \\
U^{(s)} \mathrm{x}_{s}^{(p)}
\end{array}\right),
$$

podemos concluir que

$$
\begin{aligned}
& \operatorname{Var}_{\xi_{1}}\left(\boldsymbol{Z}_{s}\right)= \\
& =\left(\begin{array}{cccc}
\operatorname{Cov}_{\xi_{1}}\left(\boldsymbol{U}^{(s)} \mathbf{y}_{s}, \boldsymbol{U}^{(s)} \mathbf{y}_{s}\right) & \operatorname{Cov}_{\xi_{1}}\left(\boldsymbol{U}^{(s)} \mathbf{y}_{s}, \boldsymbol{U}^{(s)} \mathbf{x}_{s}^{(1)}\right) & \cdots & \operatorname{Cov}_{\xi_{1}}\left(\boldsymbol{U}^{(s)} \mathbf{y}_{s}, \boldsymbol{U}^{(s)} \mathbf{x}_{s}^{(p)}\right) \\
\vdots & \vdots & \ddots & \vdots \\
\operatorname{Cov}_{\xi_{1}}\left(U^{(s)} \mathbf{x}_{s}^{(p)}, \boldsymbol{U}^{(s)} \mathbf{y}_{s}\right) & \operatorname{Cov}_{\xi_{1}}\left(\boldsymbol{U}^{(s)} \mathbf{x}_{s}^{(p)}, \boldsymbol{U}^{(s)} \mathbf{x}_{s}^{(1)}\right) & \cdots & \operatorname{Cov}_{\xi_{1}}\left(\boldsymbol{U}^{(s)} \mathbf{x}_{s}^{(p)}, U^{(s)} \mathbf{x}_{s}^{(p)}\right)
\end{array}\right)
\end{aligned}
$$


Note que

$$
\begin{aligned}
\operatorname{Cov}_{\xi_{1}}\left(\boldsymbol{U}^{(s)} \mathbf{x}_{s}^{(k)}, \boldsymbol{U}^{(s)} \mathbf{x}_{s}^{(l)}\right) & =\operatorname{Cov}_{\xi_{1}}\left[\operatorname{vec}\left(\boldsymbol{U}^{(s)} \mathbf{x}_{s}^{(k)}\right), \operatorname{vec}\left(\boldsymbol{U}^{(s)} \mathbf{x}_{s}^{(l)}\right)\right] \\
& =\operatorname{Cov}_{\xi_{1}}\left[\left(\mathbf{x}_{s}^{(k)^{\prime}} \otimes \mathbf{I}_{M_{s}}\right) \operatorname{vec}\left(\boldsymbol{U}^{(s)}\right),\left(\mathbf{x}_{s}^{(l)^{\prime}} \otimes \mathbf{I}_{M_{s}}\right) \operatorname{vec}\left(\boldsymbol{U}^{(s)}\right)\right] \\
& =\left(\mathbf{x}_{s}^{(k)^{\prime}} \otimes \mathbf{I}_{M_{s}}\right) \operatorname{Cov} \xi_{1}\left[\operatorname{vec}\left(\boldsymbol{U}^{(s)}\right), \operatorname{vec}\left(\boldsymbol{U}^{(s)}\right)\right]\left(\mathbf{x}_{s}^{(l)} \otimes \mathbf{I}_{M_{s}}\right) \\
& =\frac{1}{M_{s}-1}\left(\mathbf{x}_{s}^{(k)^{\prime}} \otimes \mathbf{I}_{M_{s}}\right)\left(\mathbf{P}_{M_{s}} \otimes \mathbf{P}_{M_{s}}\right)\left(\mathbf{x}_{s}^{(l)} \otimes \mathbf{I}_{M_{s}}\right) \\
& =\frac{1}{M-1} \mathbf{x}_{s}^{(k)^{\prime}} \mathbf{P}_{M_{s}} \mathbf{x}_{s}^{(l)} \otimes \mathbf{P}_{M_{s}} \\
& =\sigma_{s ; k, l} \mathbf{P}_{M_{s}}
\end{aligned}
$$

de modo que

$$
\operatorname{Var}_{\xi_{1}}\left(Z_{s}\right)=\Sigma_{s} \otimes \mathbf{P}_{M_{s}}
$$

Além disso, como a aleatorização no grupo $s$ é independente da aleatorização no grupo $s^{*}$,

$$
\operatorname{Var}_{\xi_{1}}(\boldsymbol{Z})=\stackrel{\oplus}{N=1}_{s}^{N}\left(\Sigma_{s} \otimes \mathbf{P}_{M_{s}}\right)
$$

\section{C.2.2 Amostragem estratificada e especificação de elementos amostrados e não amostrados}

Consideremos um processo de amostragem estratificado a partir da população de interesse, no qual escolhemos por meio de uma amostragem aleatória simples sem reposição uma amostra de tamanho $m_{s}\left(m_{s} \leq M_{s}\right)$ em cada um dos $s$ estratos, $s=1, \ldots, N$. Subdividamos o vetor $Z^{\star}$ em duas partes: uma parte amostrada (identificada pelo índice $S$ ) e outra não amostrada (identificada pelo índice $R$ ). Deste modo,

$$
\left(\begin{array}{c}
Z_{S}^{\star} \\
Z_{R}^{\star}
\end{array}\right)=\left(\begin{array}{c}
\mathrm{R}_{1} \\
\mathrm{R}_{2}
\end{array}\right) Z^{\star}
$$

em que

$$
\mathbf{R}_{i}=\stackrel{\ominus}{s=1}_{N}^{N}\left(\mathbf{I}_{p+1} \otimes \mathbf{I}_{i \bullet}^{(s)}\right) \in \mathbb{R}^{(p+1) M \times(p+1) M} \quad i=1.2
$$


e $\mathrm{I}_{i \bullet}^{(s)}, i=1,2$ vem da seguinte partição da matriz identidade $\mathbf{I}_{M_{s}}$ :

$$
\begin{aligned}
& \mathrm{I}_{1 \bullet}^{(s)}=\left(\mathrm{I}_{m_{s}}, 0_{m_{s} \times\left(M_{s}-m_{s}\right)}\right) \\
& \mathrm{I}_{2 \bullet}^{(s)}=\left(0_{\left(M_{s}-m_{s}\right) \times m_{s}}, \mathrm{I}_{M_{s}-m_{s}}\right)
\end{aligned}
$$

A partir dos momentos de $Z^{\star}$, podemos obter os momentos de $\left(\begin{array}{c}Z_{S}^{\star} \\ Z_{R}^{\star}\end{array}\right)$ como segue:

1) $E_{\xi_{1}}\left[\left(\begin{array}{c}Z_{S}^{\star} \\ Z_{R}^{\star}\end{array}\right)\right]=\left(\begin{array}{l}\mathbf{R}_{1} \\ \mathbf{R}_{2}\end{array}\right) E_{\xi_{1}}\left(Z^{\star}\right)=\left(\begin{array}{l}\mathrm{R}_{1} \\ \mathbf{R}_{2}\end{array}\right) \mathrm{F}^{\star} \mu^{(y)}=\left(\begin{array}{c}\mathrm{F}_{S}^{\star} \\ \mathrm{F}_{R}^{\star}\end{array}\right) \mu^{(y)}$, com

$$
\begin{aligned}
& \mathbf{F}_{S}^{\star}=\bigoplus_{s=1}^{N}\left(\begin{array}{c}
1_{m_{s}} \\
0_{p m_{s}}
\end{array}\right) \text { e } \quad \mathbf{F}_{R}^{\star}=\bigoplus_{s=1}^{N}\left(\begin{array}{c}
1_{M_{s}-m_{s}} \\
0_{p\left(M_{s}-m_{s}\right)}
\end{array}\right) \text {, pois } \\
& \mathbf{R}_{i} \mathbf{F}^{\star}=\left[\bigoplus_{s=1}^{N}\left(\mathbf{I}_{p+1} \otimes \mathbf{I}_{i \bullet}^{(s)}\right)\right]\left[\begin{array}{c}
N \\
\oplus=1
\end{array}\left(\begin{array}{c}
1_{M_{s}} \\
0_{p M_{s}}
\end{array}\right)\right] \\
& =\oplus_{s=1}^{N}\left[\left(\mathrm{I}_{p+1} \otimes \mathrm{I}_{i \bullet}^{(s)}\right)\left(\begin{array}{c}
1_{M_{s}} \\
0_{p M_{s}}
\end{array}\right)\right] \\
& =\oplus_{s=1}^{N}\left(\begin{array}{c}
\mathbf{I}_{i \bullet}^{(s)} 1_{M_{s}} \\
\left(\mathbf{I}_{p+1} \otimes \mathbf{I}_{i \bullet}^{(s)}\right) 0_{p \lambda M_{s}}
\end{array}\right)
\end{aligned}
$$

e $\mathbf{I}_{1 \bullet}^{(s)} 1_{M_{s}}=\left(\begin{array}{c}1_{m_{s}} \\ 0_{m_{s}}\end{array}\right), \mathbf{I}_{2 \bullet}^{(s)} 1_{M_{s}}=\left(\begin{array}{c}1_{M_{s}-m_{s}} \\ 0_{M_{s}-m_{s}}\end{array}\right), \quad\left(\mathrm{I}_{p+1} \otimes \mathrm{I}_{1 \bullet}^{(s)}\right) 0_{p M_{s}}=0_{p m_{s}} \quad$ e $\left(\mathbf{I}_{p+1} \otimes \mathbf{I}_{2 \bullet}^{(s)}\right) 0_{p M M_{s}}=0_{p\left(M_{s}-m_{s}\right)}$.

2)

$$
\begin{aligned}
\operatorname{Var}_{\xi_{1}}\left[\left(\begin{array}{c}
Z_{S}^{\star} \\
Z_{R}^{\star}
\end{array}\right)\right] & =\left(\begin{array}{cc}
\operatorname{Var}_{\xi_{1}}\left(Z_{S}^{\star}\right) & \operatorname{Cov}_{\xi_{1}}\left(Z_{S}^{\star}, Z_{R}^{\star}\right) \\
\operatorname{Cov}_{\xi_{1}}\left(Z_{R}^{\star}, Z_{S}^{\star}\right) & \operatorname{Var}_{\xi_{1}}\left(Z_{R}^{\star}\right)
\end{array}\right) \\
& =\left(\begin{array}{cc}
\mathrm{V}_{S} & \mathrm{~V}_{S R} \\
\mathrm{~V}_{R S} & \mathrm{~V}_{R}
\end{array}\right) \\
& =\left(\begin{array}{cc}
\mathrm{R}_{1} \mathrm{~V} \mathrm{R}_{1}^{\prime} & \mathrm{R}_{1} \mathrm{VR}_{2}^{\prime} \\
\mathrm{R}_{2} \mathrm{~V} \mathrm{R} & \mathrm{R}_{2}^{\prime} \mathrm{VR}_{2}^{\prime}
\end{array}\right)
\end{aligned}
$$

Para facilitar o cálculo de cada componente, consideremos as seguintes partiçōes de $\mathrm{I}_{M_{s}}, \mathrm{P}_{M_{s}}$ : 


$$
\mathbf{I}_{M_{s}}=\left(\begin{array}{l}
\mathbf{I}_{1 \bullet}^{(s)} \\
\mathbf{I}_{2 \bullet}^{(s)}
\end{array}\right)=\left(\mathbf{I}_{\bullet \mathbf{\bullet}}^{(s)}, \mathbf{I}_{\bullet 2}^{(s)}\right) \quad \text { com } \quad \begin{aligned}
& \mathbf{I}_{1 \bullet}^{(s)}=\left(\mathbf{I}_{m_{s}}, 0_{m_{s} \times\left(M_{s}-m_{s}\right)}\right)=\mathbf{I}_{\bullet \mathbf{1}}^{(s)^{\prime}} \\
& \mathbf{I}_{2 \bullet}^{(s)}=\left(0_{\left(M_{s}-m_{s}\right) \times m_{s}}, \mathbf{I}_{M_{s}-m_{s}}\right)=\mathbf{I}_{\bullet 2}^{(s)^{\prime}}
\end{aligned}
$$

e

$$
\mathbf{P}_{M_{s}}=\left(\begin{array}{ll}
\mathbf{P}_{11}^{(s)} & \mathbf{P}_{12}^{(s)} \\
\mathbf{P}_{21}^{(s)} & \mathbf{P}_{22}^{(s)}
\end{array}\right) \quad \text { com } \quad \begin{aligned}
& \mathbf{P}_{11}^{(s)}=\mathbf{I}_{m_{s}}-M_{s}{ }^{-1} \mathbf{J}_{m_{s}} \\
& \mathbf{P}_{12}^{(s)}=-M_{s}{ }^{-1} \mathbf{J}_{m_{s} \times\left(M_{s}-m_{s}\right)}=\mathbf{P}_{21}^{(s)^{\prime}} \\
& \mathbf{P}_{22}^{(s)}=\mathbf{I}_{M_{s}-m_{s}}-M_{s}{ }^{-1} \mathbf{J}_{M_{s}-m_{s}}
\end{aligned}
$$

Note que para $i, j=1,2$

$$
\begin{aligned}
& \mathbf{R}_{i} \mathrm{~V} \mathbf{R}_{j}^{\prime}=\left[\underset{\oplus=1}{N}\left(\mathbf{I}_{p+1} \otimes \mathbf{I}_{i \bullet}^{(s)}\right)\right]\left[\underset{\oplus=1}{N}\left(\Sigma_{s} \otimes \mathbf{P}_{M_{s}}\right)\right]\left[\underset{s=1}{\oplus}\left(\mathbf{I}_{p+1} \otimes \mathbf{I}_{\bullet j}^{(s)}\right)\right] \\
& =\oplus_{s=1}^{N}\left[\Sigma_{s} \otimes\left(\mathbf{I}_{i \bullet}^{(s)} \mathbf{P}_{M_{s}} \mathbf{I}_{\bullet j}^{(s)}\right)\right]
\end{aligned}
$$

$\operatorname{com} \quad \mathbf{I}_{i \bullet}^{(s)} \mathbf{P}_{M_{s}} \mathbf{I}_{\bullet j}^{(s)}=\mathbf{P}_{i j}^{(s)}$, de modo que

$$
\begin{gathered}
\mathrm{V}_{S}=\bigoplus_{s=1}^{N}\left[\Sigma_{s} \otimes\left(\mathbf{I}_{m_{s}}-M_{s}^{-1} \mathbf{J}_{m_{s}}\right)\right] \\
\mathrm{V}_{S R}=\bigoplus_{s=1}^{N}\left[\Sigma_{s} \otimes\left(-M_{s}\right)^{-1} \mathbf{J}_{m_{s} \times\left(M_{s}-m_{s}\right)}\right]=\mathrm{V}_{R S}^{\prime}
\end{gathered}
$$

e

$$
\mathrm{V}_{R}=\underset{s=1}{\stackrel{N}{\oplus}}\left[\Sigma_{s} \otimes\left(\mathrm{I}_{M_{s}-m_{s}}-M_{s}^{-1} \mathbf{J}_{M_{s}-m_{s}}\right)\right]
$$

\section{C.2.3 Estimação da resposta total em cada estrato usando informação das covariáveis}

Calculemos cada componente de

$$
\widehat{T}^{(y)}=\left[\mathrm{V}_{S}^{-1} \mathrm{~F}_{S}^{\star}\left(\mathbf{F}_{S}^{\star \prime} \mathrm{V}_{S}^{-1} \mathbf{F}_{S}^{*}\right)^{-1}\left(\mathrm{~F}_{S}^{\star \prime} \mathrm{L}_{S}+\mathrm{F}_{R}^{\star \prime} \mathrm{L}_{R}\right)\right]^{\prime} Z_{S}^{*}
$$

Primeiramente,

$$
\mathrm{V}_{S}^{-1}=\bigodot_{s=1}^{\mathrm{V}}\left[\Sigma_{s}^{-1} \otimes\left(\mathrm{I}_{m_{s}}-M_{s}^{-1} \mathrm{~J}_{m_{s}}\right)^{-1}\right]
$$


em que

$$
\Sigma_{s}^{-1}=\left(\begin{array}{cc}
\sigma_{s ; y}^{2} & \sigma_{s ; y, X} \\
\sigma_{s ; X, y} & \Sigma_{s ; X}
\end{array}\right)^{-1}=\left(\begin{array}{cc}
\Psi_{11}^{(s)} & \Psi_{12}^{(s)} \\
\Psi_{21}^{(s)} & \Psi_{22}^{(s)}
\end{array}\right)
$$

com

$$
\begin{gathered}
\Psi_{11}^{(s)}=\left(\sigma_{s ; y}^{2}-\sigma_{s ; y, X} \Sigma_{s ; X}^{-1} \sigma_{s ; X, y}\right)^{-1} \\
\Psi_{12}^{(s)}=-\frac{\sigma_{s ; y, X}}{\sigma_{s ; y}^{2}}\left(\Sigma_{s ; X}-\frac{1}{\sigma_{s ; y}^{2}} \sigma_{s ; X, y} \sigma_{s ; y, X}\right)^{-1} \\
\Psi_{21}^{(s)}=-\left(\Sigma_{s ; X}-\frac{\sigma_{s ; X, y} \sigma_{s ; y, X}}{\sigma_{s ; y}^{2}}\right)^{-1} \frac{\sigma_{s ; X, y}}{\sigma_{s ; y}^{2}} \\
\Psi_{22}^{(s)}=\left(\Sigma_{s ; X}-\frac{\sigma_{s ; X, y} \sigma_{s ; y, X}}{\sigma_{s ; y}^{2}}\right)^{-1}
\end{gathered}
$$

$\mathrm{e}$

$$
\left(\mathbf{I}_{m_{s}}-M_{s}^{-1} \mathbf{J}_{m_{s}}\right)^{-1}=\mathrm{I}_{m_{s}}+\frac{1}{M_{s}-m_{s}} \mathbf{J}_{m_{s}} .
$$

Como

$$
\left(\mathbf{I}_{m_{s}}-M_{s}^{-1} \mathbf{J}_{m_{s}}\right)^{-1} \mathbf{1}_{m_{s}}=\frac{M_{s}}{M_{s}-m_{s}} 1_{m_{s}}
$$

obtemos que

$$
\begin{aligned}
& \mathrm{V}_{s}^{-1} \mathrm{~F}_{S}^{\star}=\left\{\stackrel{N}{\oplus}\left[\Sigma_{s=1}^{-1} \otimes\left(\mathbf{I}_{m_{s}}-M_{s}^{-1} \mathbf{J}_{m_{s}}\right)^{-1}\right]\right\}\left[\begin{array}{c}
\stackrel{N}{\oplus} \\
\oplus_{s=1}
\end{array}\left(\begin{array}{c}
1_{m_{s}} \\
0_{p m_{s}}
\end{array}\right)\right] \\
& =\bigoplus_{s=1}^{N}\left\{\left[\Sigma_{s}^{-1} \otimes\left(\mathrm{I}_{m_{s}}-M_{s}^{-1} \mathbf{J}_{m_{s}}\right)^{-1}\right]\left(\begin{array}{c}
1_{m_{s}} \\
0_{p m_{s}}
\end{array}\right)\right\} \\
& =\bigoplus_{s=1}^{N} \frac{M_{s}}{M_{s}-m_{s}}\left(\begin{array}{c}
\Psi_{11}^{(s)} 1_{m_{s}} \\
\Psi_{21}^{(s)} \otimes 1_{m_{s}}
\end{array}\right)
\end{aligned}
$$

Denotando

$$
\rho_{s: y}^{2}=\frac{\sigma_{s: y . X} \Sigma_{s ; X}^{-1} \sigma_{s: X, y}}{\sigma_{s: y}^{2}}
$$

segue que

$$
\Psi_{11}^{(s)}=\left[\sigma_{s ; y}^{2}\left(1-\rho_{s: y}^{2}\right)\right]^{-1}
$$

e como

$$
\left(\Sigma_{s: X}-\frac{\sigma_{s: X: Y, y} \sigma_{s: y . X}}{\sigma_{s: y}^{2}}\right)^{-1}=\Sigma_{s: X}^{-1}+\frac{\Sigma_{s: Y}^{-1} \sigma_{s: X, y} \sigma_{s: y, X} \Sigma_{s: X}^{-1}}{\sigma_{s: y}^{2}\left(1-\rho_{s: y}^{2}\right)}
$$


segue que

$$
\begin{aligned}
\Psi_{21}^{(s)} & =-\frac{1}{\sigma_{s ; y}^{2}} \Sigma_{s ; X^{-1}}^{-1} \sigma_{s ; X, y}-\frac{\rho_{s ; y}^{2}}{\sigma_{s ; y}^{2}\left(1-\rho_{s ; y}^{2}\right)} \Sigma_{s ; X}^{-1} \sigma_{s ; X, y} \\
& =-\frac{1}{\sigma_{s ; y}^{2}\left(1-\rho_{s ; y}^{2}\right)} \Sigma_{s ; X}^{-1} \sigma_{s ; X, y}
\end{aligned}
$$

Portanto,

$$
\begin{aligned}
& \mathbf{V}_{s}^{-1} \mathbf{F}_{S}^{\star}=\bigoplus_{s=1}^{N} \frac{M_{s}}{M_{s}-m_{s}}\left[\sigma_{s ; y}^{2}\left(1-\rho_{s ; y}^{2}\right)\right]^{-1}\left(\begin{array}{c}
1_{m_{s}} \\
-\Sigma_{s ; X^{-}}^{-1} \sigma_{s ; X ; y} \otimes 1_{m_{s}}
\end{array}\right) \\
& =\underset{s=1}{N} \frac{M_{s}}{M_{s}-m_{s}}\left[\sigma_{s ; y}^{2}\left(1-\rho_{s ; y}^{2}\right)\right]^{-1}\left(\begin{array}{c}
\mathbf{1}_{m_{s}} \\
-\boldsymbol{\beta}_{s ; y} \otimes \mathbf{1}_{m_{s}}
\end{array}\right)
\end{aligned}
$$

Por outro lado,

de modo que

$$
\begin{aligned}
& \mathbf{F}_{S}^{\star \prime} \mathbf{V}_{s}^{-1} \mathbf{F}_{S}^{\star}=\left[\bigoplus_{s=1}^{N}\left(1_{m_{s}}^{\prime}, 0_{p m_{s}}^{\prime}\right)\right]\left[\underset{\oplus=1}{N} \frac{M_{s}}{M_{s}-m_{s}}\left(\begin{array}{c}
\Psi_{11}^{(s)} 1_{m_{s}} \\
\Psi_{21}^{(s)} \otimes 1_{m_{s}}
\end{array}\right)\right] \\
& =\bigoplus_{s=1}^{N} \frac{M_{s}}{M_{s}-m_{s}}\left(\mathbf{1}_{m_{s}}^{\prime} \Psi_{11}^{(s)} 1_{m_{s}}\right) \\
& =\bigoplus_{s=1}^{N} \frac{m_{s} M_{s}}{M_{s}-m_{s}} \Psi_{11}^{(s)}
\end{aligned}
$$

$$
\left(\mathrm{F}_{S}^{\star \prime} \mathrm{V}_{s}^{-1} \mathbf{F}_{S}^{\star}\right)^{-1}=\bigoplus_{s=1}^{N} \frac{M_{s}-m_{s}}{m_{s} M_{s}}\left[\Psi_{11}^{(s)}\right]^{-1}=\bigoplus_{s=1}^{N} \frac{M_{s}-m_{s}}{m_{s} M_{s}} \sigma_{s ; y}^{2}\left(1-\rho_{s: y}^{2}\right)
$$

É fácil mostrar que

$$
\mathbf{F}_{S}^{\star \prime} \mathbf{L}_{S}=\bigoplus_{s=1}^{N} m_{s} \quad \text { e } \quad \mathbf{F}_{R}^{\star \prime} \mathbf{L}_{R}=\bigoplus_{s=1}^{N}\left(M_{s}-m_{s}\right)
$$

de modo que

$$
\mathrm{F}_{S}^{\star \prime} \mathbf{L}_{S}+\mathbf{F}_{R}^{\star \prime} \mathbf{L}_{R}=\underset{s=1}{\stackrel{N}{\oplus}} M_{s}
$$

Finalmente,

$$
\begin{aligned}
& \mathbf{V}_{S}^{-1} \mathbf{F}_{S}^{\star}\left(\mathbf{F}_{S}^{\star \prime} \mathbf{V}_{S}^{-1} \mathbf{F}_{S}^{\star}\right)^{-1}\left(\mathbf{F}_{S}^{\star \prime} \mathbf{L}_{S}+\mathbf{F}_{R}^{\star \prime} \mathbf{L}_{R}\right)= \\
&=\left[\bigoplus_{s=1}^{N} \frac{M_{s}}{M_{s}-m_{s}}\left[\sigma_{s: y}^{2}\left(1-\rho_{s: y}^{2}\right)\right]^{-1}\left(\begin{array}{c}
1_{m_{s}} \\
-\boldsymbol{\beta}_{s: y} \otimes 1_{m_{s}}
\end{array}\right)\right]\left[\bigoplus_{s=1}^{N} \frac{M_{s}-m_{s}}{m_{s} M_{s}} \sigma_{s: y}^{2}\left(1-\rho_{s: y}^{2}\right)\right] \times \\
& {\left[\bigoplus_{s=1}^{N} M_{s}\right] } \\
&= \underset{s=1}{N} \frac{M_{s}}{m_{s}}\left(\begin{array}{c}
1_{m_{s}} \\
-\beta_{s: y} \otimes 1_{m_{s}}
\end{array}\right)
\end{aligned}
$$


Por conseguinte,

$$
\begin{aligned}
\widehat{T}^{(y)} & =\left[\mathrm{V}_{S}^{-1} \mathrm{~F}_{S}^{\star}\left(\mathbf{F}_{S}^{\star \prime} \mathrm{V}_{S}^{-1} \mathbf{F}_{S}^{\star}\right)^{-1}\left(\mathrm{~F}_{S}^{\star \prime} \mathrm{L}_{S}+\mathrm{F}_{R}^{\star \prime} \mathrm{L}_{R}\right)\right]^{\prime} Z_{S}^{\star} \\
= & \left(\begin{array}{c}
M_{1}\left[\bar{Y}_{S 1}-\sum_{k=1}^{p} \beta_{1 ; y ; k}\left(\bar{X}_{S 1}^{(k)}-\mu_{1}^{(k)}\right)\right] \\
\vdots \\
M_{N}\left[\bar{Y}_{S N}-\sum_{k=1}^{p} \beta_{N ; y ; k}\left(\bar{X}_{S N}^{(k)}-\mu_{N}^{(k)}\right)\right]
\end{array}\right)
\end{aligned}
$$

com

$$
\begin{gathered}
\beta_{s ; y}=\left(\beta_{s ; y, 1}, \ldots, \beta_{s ; y, p}\right)^{\prime}=\Sigma_{s ; X}^{-1} \sigma_{s ; X y} \\
\bar{Y}_{\dot{S}}=\left(\bar{Y}_{S 1}, \ldots, \bar{Y}_{S N}\right)^{\prime}=\left[\bigoplus_{s=1}^{N} \frac{1}{m_{s}} 1_{m_{S}}^{\prime}\right] Y_{S}
\end{gathered}
$$

e

$$
\bar{X}_{S}^{(k)}=\left(\bar{X}_{S 1}^{(k)}, \ldots, \bar{X}_{S N}^{(k)}\right)^{\prime}=\left[\bigoplus_{s=1}^{N} \frac{1}{m_{s}} 1_{m_{S}}^{\prime}\right] X_{S}^{(k)} \quad k=1, \ldots, p
$$




\section{Referências Bibliográficas}

[1] Battese, G.E., Harter, R.M. and Fuller, W.A. (1988). An error-components model for prediction of county areas using survey and satellite data. Journal of the American Statistical Association, 83, 28-36.

[2] Bolfarine, H. e Bussab, W.O. (2005). Elementos de amostragem. São Paulo: Editora Edgard Blücher.

[3] Bolfarine, H. and Zacks, S. (1992). Prediction Theory for Finite Populations. New York: Springer-Verlag.

[4] Breslow, N.E. and Clayton, D.G. (1993). Approximate inference in generalized linear mixed models. Journal of the American Statistical Association, 88, 9-25.

[5] Breslow, N.E. and Lin, X. (1995). Bias correction in generalised linear mixed models with a single component of dispersion. Biometriki, 82, 81-91.

[6] Brewer, K.R.W. (1999). Design-based or prediction-based inference? Stratified random vs stratified balanced sampling. International Statistical Review, 67 , $35-47$.

[7] Brewer, K.R.W., Hanif, M. and Tam, S.M. (1988). How nearly can model-based prediction and design-based estimation be reconciled? Journal of the Americun Statistical Association, 83, 128-132.

[8] Cassel, C.M., Särndal, C.E. and Wretman, J.H. (1977). Foundations of Inference in Survey Sampling. New York: John Wiley it Sons.

[9] Cochran, W.G. (1977). Sampling Techniques (3rd ed.). New York: John Wiley \& Sons. 
[10] Das, K., Jiang, J. and Rao, J.N.K. (2004). Mean squared error of empirical predictor. The Annals of Statistics, 32, 818-840.

[11] Dempster, A.P., Rubin, D.B. and Tsutakawa, R.K. (1981). Estimation in covariance component models. Journal of the American Statistical Association, 76, 341-353.

[12] Fahrmeir, L. and Tutz, G. (1994). Multivariate statistical modelling based on. generalized linear models. New York: Springer-Verlag.

[13] Fay, R.E. and Herriot, R.A. (1979). Estimates of income for small places: and application of James-Stein procedures to census data. Journal of the American. Statistical Association, 74, 269-277.

[14] Goldberger, A.S. (1962). Best linear unbiased prediction in the generalized linear regression model. Journal of the American Statistical Association, 57, 369-375.

[15] Kackar, R.N. and Harville, D.A. (1984). Approximations of standard errors of estimators of fixed and random effects in mixed linear models. Journal of the American Statistical Association, 79, 853-863.

[16] Lencina, V.B. (2002). Modelos de efeitos aleatórios e populaçôes finitas. Tese de Doutorado. São Paulo: Instituto de Matemática e Estatística, Universidade de São Paulo.

[17] Li, W. (2003). Use of Random permutation model in rate estimation and standardization. Ph.D. Dissertation. Massachusetts: Graduate School of the University of Massachusetts Amherst.

[18] Li, W. and Stanek, E.J.III (2005). Design-based random permutation models with auxiliary information. Submetido ao Journal of Statistical Planning and. Inference.

[19] Lin, X. and Breslow, N.E. (1996). Bias correction in generalized linear mixed models with multiple components of dispersion. Journal of the American Statistical Association, 91, 1007-1016. 
[20] Littell, R.C., Milliken, G.A., Stroup, W.W. and Wolfinger, R.D. (1996). SAS ${ }^{\circledR}$ System for Mixed Models. Caro, NC: SAS Institute Inc.

[21] Little, R.J. (2004). To model or not to model? Competing Modes of inference for finite population sampling. Journal of the American Statistical Association, 99, 546-556.

[22] McCulloch, C.E. and Searle, S.R. (2001). Generalized, linear and mixed models. New York: John Wiley \& Sons.

[23] Prasad, N.G.N. and Rao, J.N.K. (1990). The Estimation of the Mean Squared Error of Small-Area Estimators. Journal of the American Statistical Association, 85, 163-171.

[24] Rao, C.R. and Toutenburg, H. (1999). Linear Models. New York: SpringerVerlag.

[25] Robinson, G.K. (1991). That BLUP is a good thing: the estimation of random effects. Statistical Science, 6, 15-51.

[26] Särndal, C.E., Swensson, B. and Wretman, J. (1992). Model Assisted Survey Sampling. New York: Springer-Verlag.

[27] Schall, R. (1991). Estimation in generalized linear models with random effects. Biometrika, 78, 719-727.

[28] Scott, A. and Smith, T.M. (1969). Estimation in Multi-Stage Surveys. Journal of the American Statistical Association, 64, 830-840.

[29] Searle, S.R, Casella, G. and Mc Culloch. C.E. (1992). Variance Components. New York: John Wiley and Sons.

[30] Searle, S.R. and Fawcett, R. F. (1970). Expected Mean Squares in Variance Components Models Having Finite Populations. Biometrics, 26, 243-254.

[31] Seber, G.A.F (1984). Multivariate observations. New York: Joln Wiley \& Sons.

[32] Srivasta. V.K. and Giles, D.E.E. (1987). Seemingly Unrelated Regression Equations Models. New York: Marcel Dekker. 
[33] Stanek, E.J.III, Singer, J.M. (2004). Predicting Random Effects from Finite Population Clustered Samples with Response Error. Journal of the American Statistical Association, 99, 1119-1130.

[34] Stanek, E.J.III, Singer, J.M. and Lencina, V.B. (2004). A Unified Approach to Estimation and Prediction under Simple Random Sampling. Journal of Statistical Planning and Inference, 121, 325-338.

[35] Subramani, J. (1991). On invariant quadratic unbiased estimation of variance components. Communications in Statistics Theory and Methods, 20, 1705-1730.

[36] Valliant, R., Dorfman, A.H. and Royall, R.M. (2000). Finite Population Sampling and Inference. New York: John Wiley \& Sons.

[37] Waclawiw, M.A. and Liang, K. (1993). Prediction of random effects in the generalized linear model. Journal of the American Statistical Association, 88, 171-178.

[38] Wolfinger, R. and O'Connell, M. (1993). Generalized linear mixed models: A pseudo-likelihood approach. Journal of Statistical Computation and Simulation, 48, 233-243.

[39] Zeger, S.L. and Karim, M.R. (1991). Generalized linear models with random effects; A Gibbs sampling approach. Journal of the American Statistical Association, 86, 79-86.

[40] Zellner, A. (1963). Estimators for seemingly unrelated regression equations: some exact finite sample results. Journal of the American Statistical Association, 58, 977-992. 